\title{
Remediation of Spatial Skills in First Semester Calculus Using Haptics-Based Applications
}

\author{
Kristen L. Murphy \\ West Virginia University, kmurph14@mix.wvu.edu
}

Follow this and additional works at: https://researchrepository.wvu.edu/etd

Part of the Mathematics Commons

\section{Recommended Citation \\ Murphy, Kristen L., "Remediation of Spatial Skills in First Semester Calculus Using Haptics-Based Applications" (2021). Graduate Theses, Dissertations, and Problem Reports. 10206. \\ https://researchrepository.wvu.edu/etd/10206}

This Dissertation is protected by copyright and/or related rights. It has been brought to you by the The Research Repository @ WVU with permission from the rights-holder(s). You are free to use this Dissertation in any way that is permitted by the copyright and related rights legislation that applies to your use. For other uses you must obtain permission from the rights-holder(s) directly, unless additional rights are indicated by a Creative Commons license in the record and/ or on the work itself. This Dissertation has been accepted for inclusion in WVU Graduate Theses, Dissertations, and Problem Reports collection by an authorized administrator of The Research Repository @ WVU.

For more information, please contact researchrepository@mail.wvu.edu. 


\title{
REMEDIATION OF SPATIAL SKILLS IN FIRST SEMESTER CALCULUS USING HAPTICS-BASED APPLICATIONS
}

\author{
Kristen L. Murphy \\ Dissertation submitted \\ to the Eberly College of Arts and Sciences \\ at West Virginia University \\ in partial fulfillment of the requirements for the degree of
}

Doctor of Philosophy in

Mathematics

\author{
Marjorie Darrah, Ph.D., Chair \\ Nicole Engelke Infante, Ph.D. \\ Michael Mays, Ph.D. \\ Michelle Richards-Babb, Ph.D. \\ Vicki Sealey, Ph.D. \\ Department of Mathematics
}

\author{
Morgantown, West Virginia \\ 2021
}

Keywords: haptic technology, haptics, spatial reasoning, spatial skills

Copyright 2021 Kristen L. Murphy 


\section{ABSTRACT \\ Remediation of Spatial Skills in First Semester Calculus Using Haptics-Based Applications}

\section{Kristen L. Murphy}

Spatial reasoning is required for many topics in undergraduate mathematics as well as other STEM fields. In calculus specifically, there are few interventions that assess students' spatial skills and provide remediation. Haptic technology is a novel approach to spatial skills remediation due to its unfamiliarity to students and its flexibility with regard to models. The purpose of this dissertation is two-fold: first, to determine what level of spatial abilities students possess upon entering an undergraduate calculus course, and second, to determine whether haptic feedback will enhance an intervention for improving spatial skills.

The dissertation research was a mixed-methods study using the combination of a quantitative spatial reasoning test, semi-structured interviews, and student activities. The design of the study was pre-test - post-test matched subject, using the quantitative spatial reasoning test - the Santa Barbara Solids Test (SBST) - and other demographic information to match students of similar spatial reasoning abilities and backgrounds for the qualitative part of the study. In all, 306 students completed both the SBST pre-test and post-test. Ten of these students were selected as matched pairs, of which nine completed the pre-interview, intervention activities, and postinterview, leaving eight students in four matched pairs for the qualitative analyses.

Analysis of the pre-test and post-test scores showed that the students who completed the intervention activities performed significantly better on the SBST post-test than on the pre-test. Furthermore, these students improved their scores more than the students who did not complete the intervention activities, and the difference between these groups was significant. While some differences were noted between the students who completed the intervention activities with haptic feedback and those who completed them without, there were not significant differences in performance on the post-test, nor the improvement from the pre-test to the post-test.

The pre-interviews and post-interviews were coded using the spatial skills framework created by Engelke et al. (2016). Examination of the codes applied to each set of interviews showed a similar ratio of codes in each set. Both interviews contained an overwhelming majority of visualization language over orientation language. The most prominent subcategory displayed was representing objects, followed by structuring, mathematical properties, and finally measurement. The students who completed the intervention activities with haptic feedback and those who completed them without showed similar changes in their spatial language between the pre-interview and post-interview. Analysis of the scores of the interview questions did not show a significant difference between the pre-interviews and post-interviews, nor did it show a significant difference between the students who completed the intervention activities with haptic feedback and those who completed them without.

These findings indicate that students entering calculus have strong visualization and representing objects spatial skills, but lack orientation and structuring spatial skills. They also show that putting students through a short remediation, even without feedback on their performance, can improve spatial skills. Although significant differences could not be found between the students who received haptic feedback in the intervention activities and those who did not, this could be due to the small number of matched pairs, and does not rule out the potential for haptic feedback use in spatial reasoning training. 


\section{DEDICATION}

To Dad -

I wish you were here to see the final product.

To Lucy and Gabby -

Never stop chasing your dreams. 


\section{ACKNOWLEDGEMENTS}

The completion of this project would not have been possible without the help and support of many people along the way. I will attempt to give the proper amount of thanks here, knowing that no combination of words can truly express how deeply appreciative I am to all of you.

First, to my advisor, Dr. Marjorie Darrah. Thank you for not giving up on me. This process was a long, drawn out one, with many speed bumps along the way. Your patience and understanding are much appreciated. Thank you for introducing me to the wonderful world of haptics and for encouraging me to stay on track, even if the train was moving ever so slowly. You have gone above and beyond and I cannot thank you enough.

To the rest of my committee members, thank you for hanging in there and staying on board. Your expertise and guidance did wonders to help steer this project in the right direction. To Karl Mueller, thank you for proctoring the intervention meetings. You saved me from spending some long days pulling double duty with work and research, and for that I am grateful.

This research would not have been what it was without the use of the Santa Barbara Solids Test and the Novint Falcon. Thank you to Cheryl Cohen and Mary Hegarty for making your spatial reasoning test readily available for use in research. Thank you to Walt Aviles for the work done on the Falcon, for providing the appropriate libraries to make it function, and for working diligently to fulfill requests for new functionality. I have enjoyed working with your product.

To the many teachers I have had along the way, thank you for teaching me to love learning. You all made the experience so enjoyable that I never wanted to stop. Special thanks are due to Mr. Ted LaRue for convincing me to "stay broke" - may you know how crucial that conversation was to putting me on this path. 
Renee, your friendship through this process has been amazing. There is not a version of grad school that I would want to complete without you in it. Thank you for collaborative homework sessions, for letting me crash at your apartment, for bringing me back to reality when I go to the land of worst-case scenarios, and for putting up with my criticisms of your lack of pop-culture knowledge. Remember, H is big. To my many other WVU classmates: Janet, Kristin, Brittany, Martin, Todd, and John, to name a few - thank you for keeping class time enjoyable and for the many, many memories made in-between those times.

Mom, thank you for your not-so-subtle reminders that I needed to get this done because I made a promise to do so. Thank you for always supporting me in this dream and for being a constant source of encouragement. Most of all, thank you for just being a wonderful mom. I hope I make you proud. To my girls, thank you for being you. You are by far my greatest accomplishment. Thank you for the constant questions about what I've been working on and the utter confusion about why Mom is in school. May it all make sense for you one day.

And finally, Cody. I have saved you until last because otherwise I would have exhausted my words with all the thanks I have for you. Thank you for putting up with my late nights and this crazy idea I had about getting this degree. Thank you for being an awesome dad and taking over morning duties after late-night writing sessions. Thank you for all the love and support you have given me over the last fifteen years. I do not want to go through this life with anyone else by my side. Here's to the many years we have left of you honking the horn every time I walk by the car. I love you always. 


\section{TABLE OF CONTENTS}

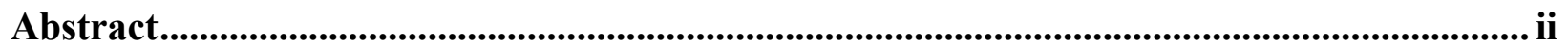

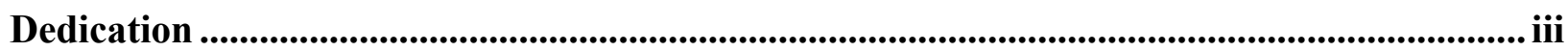

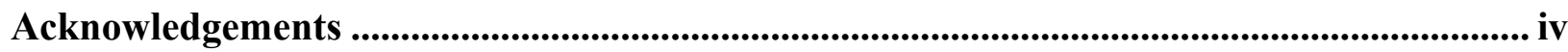

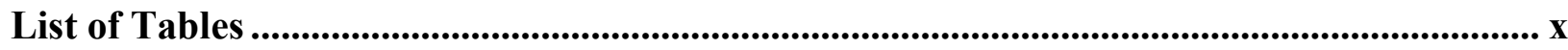

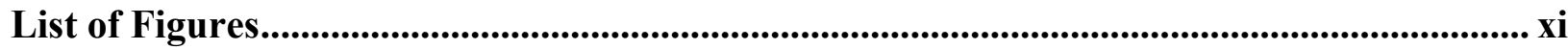

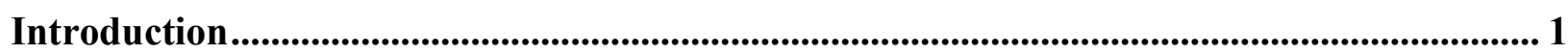

Definitions and Literature Review ......................................................................................... 3

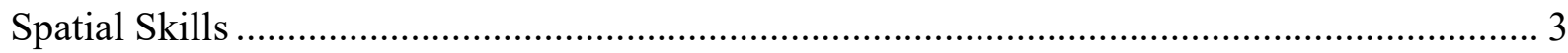

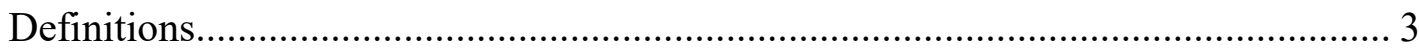

Literature Review on Training for Spatial Skills ............................................. 5

Literature Review on Definite Integrals ....................................................... 7

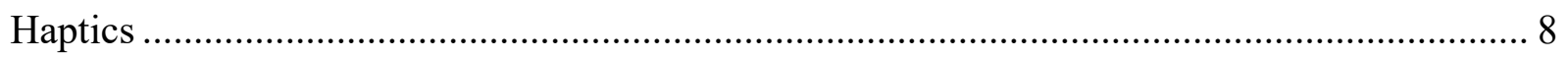

Literature Review on Haptic Feedback used in Education .................................... 9

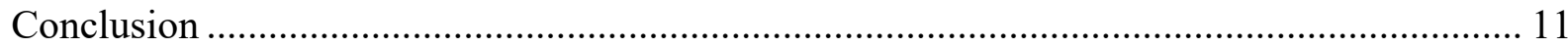

Research Questions ....................................................................................................................... 13

Research Question 1: What Spatial Skills Related to Cross-Sections Do Students Entering

Calculus Possess? ....................................................................................... 13

Research Question 2: What Difference in Performance Does the Haptic Group Exhibit Over the Non-Haptic Group in the Post-Interview? 
Research Question 3: How Do the Performance and Explanations Differ Among the Interview Population From the Pre-Interview to the Post-Interview? ..................................... 15

Research Question 4: How Does the Difference in the Pre/Post Spatial Skills Test Given at the Beginning and the End of the Semester Differ Among the Groups?........................ 15

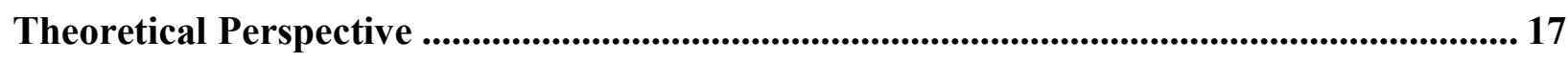

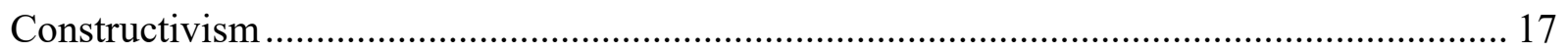

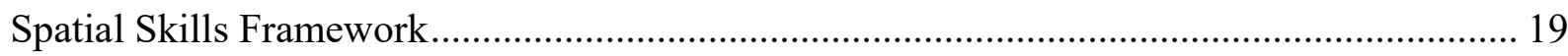

Theoretical Perspective for Remediation of Spatial Skills ............................................. 22

Spatial Reasoning Tests............................................................................................................. 23

Santa Barbara Solids Test ...................................................................................... 23

Spatial Reasoning Aptitude Test - Hard..................................................................... 25

Revised Purdue Spatial Visualization Tests: Visualization of Rotations ............................ 25

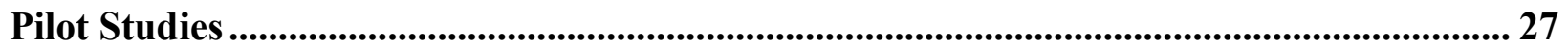

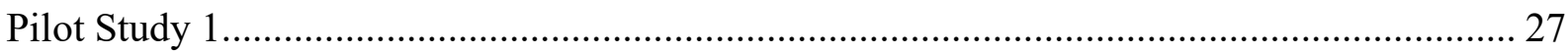

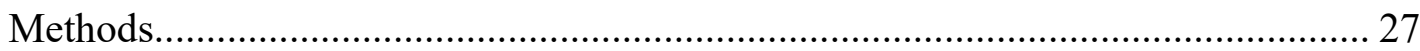

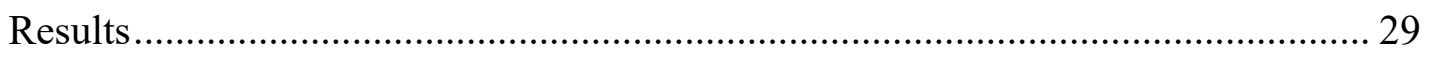

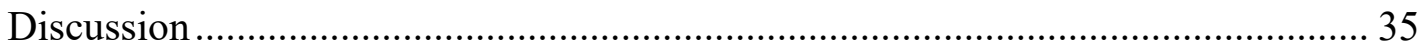

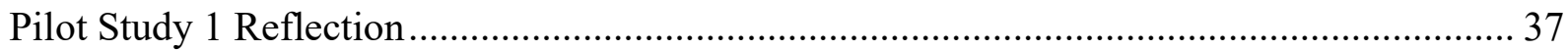

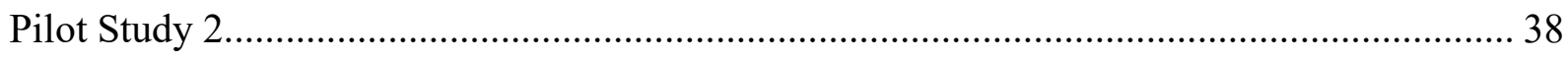

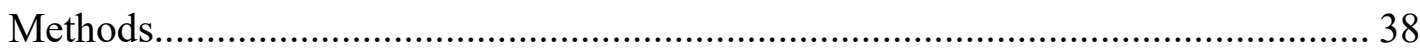

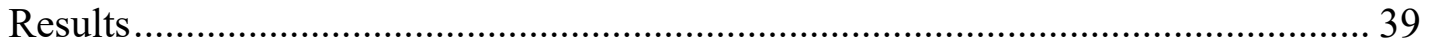


Discussion

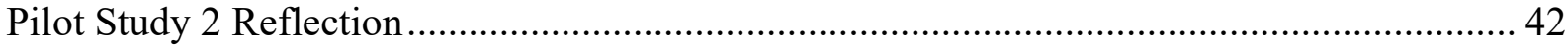

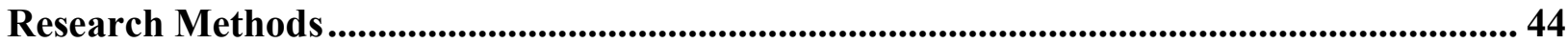

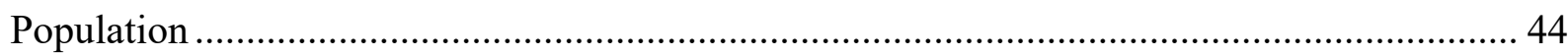

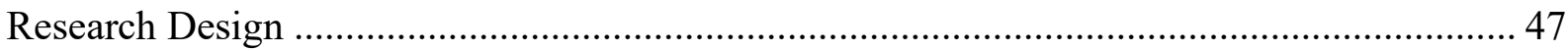

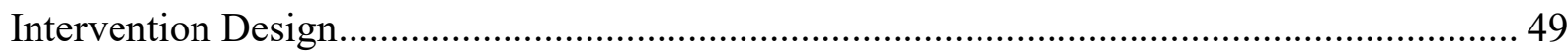

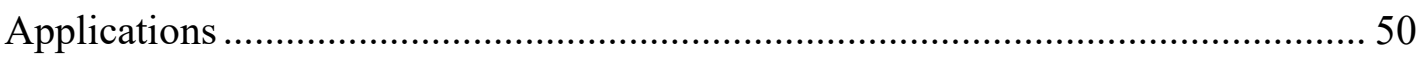

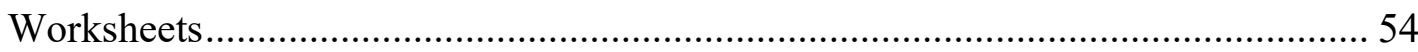

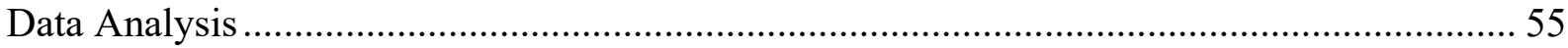

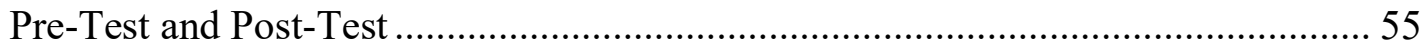

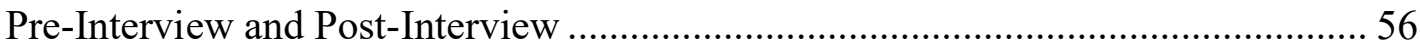

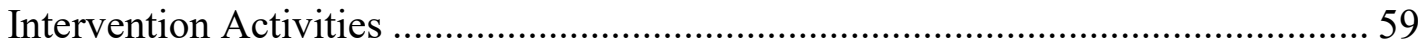

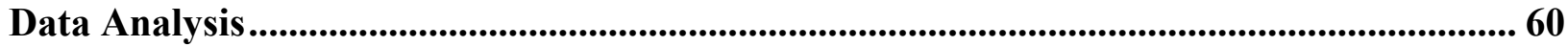

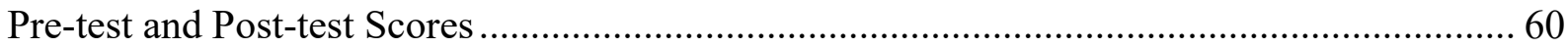

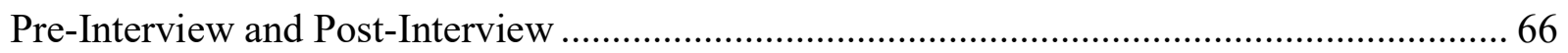

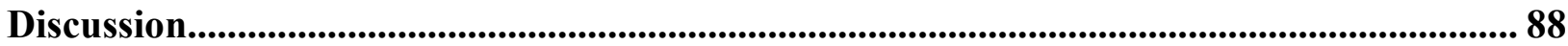

Research Question 1: What Spatial Skills Related to Cross-Sections Do Students Entering

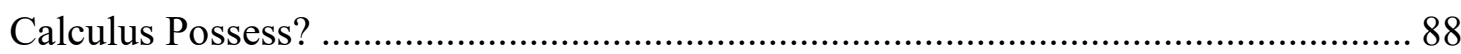

Research Question 2: What Difference in Performance Does the Haptic Group Exhibit Over the Non-Haptic Group in the Post-Interview? 
Research Question 3: How Do the Performance and Explanations Differ Among the Interview Population From the Pre-Interview to the Post-Interview? 92

Research Question 4: How Does the Difference in the Pre/Post Spatial Skills Test Given at the Beginning and the End of the Semester Differ Among the Groups?......................... 93

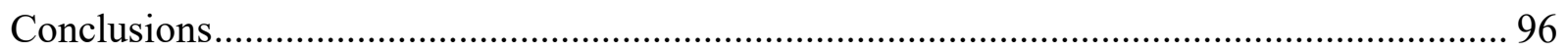

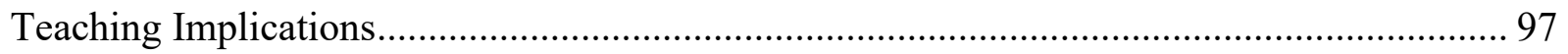

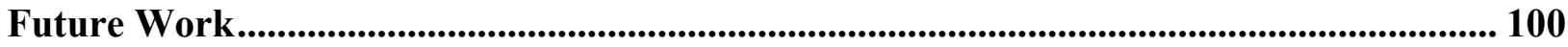

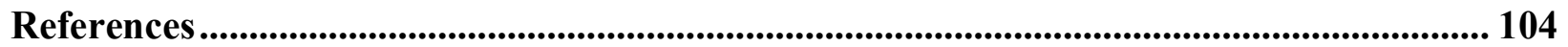

Appendix A: Santa Barbara Solids Test ........................................................................................ 109

Appendix B: Spatial Reasoning Aptitude Test - Hard ......................................................... 129

Appendix C: Revised Purdue Spatial Visualization Test: Rotations .................................... 134

Appendix D: Pilot Study 1 Solids of Revolution Problems ................................................ 172

Appendix E: Pilot Study 2 Interview Problems .......................................................... 173

Appendix F: Interview Protocol ............................................................................................. 174

Appendix G: Application Directions - With Haptics ....................................................... 176

Appendix H: Application Directions - Without Haptics..................................................... 177

Appendix I: Sample Activity ................................................................................................. 178 


\section{LIST OF TABLES}

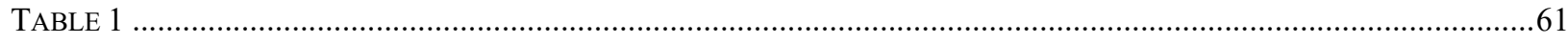

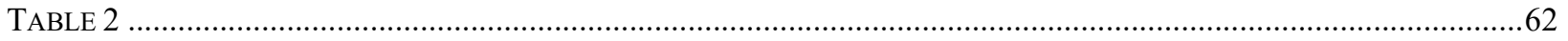

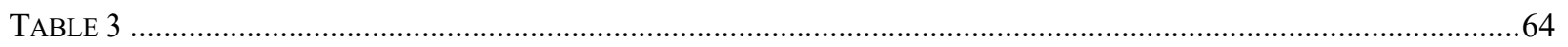

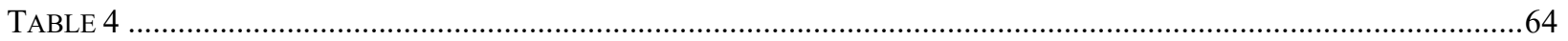

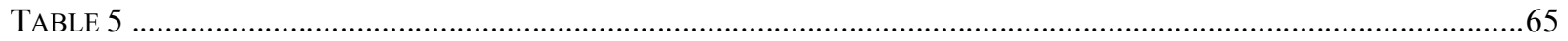

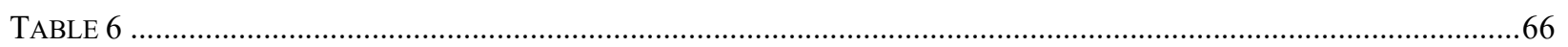

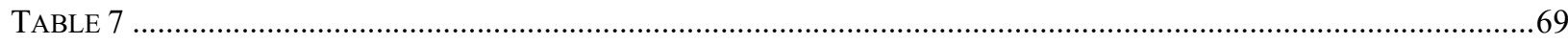

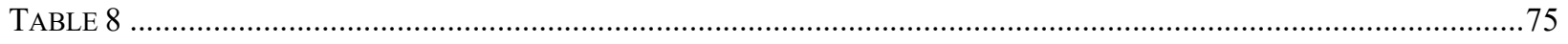

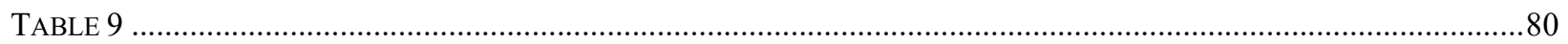

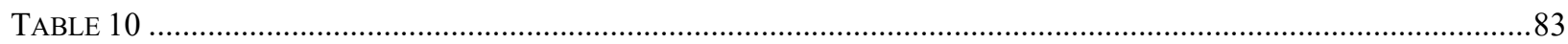

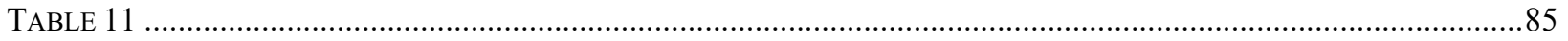

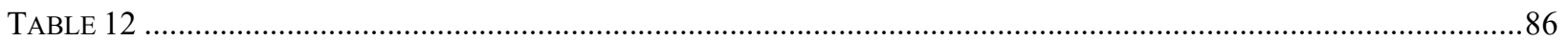




\section{LIST OF FIGURES}

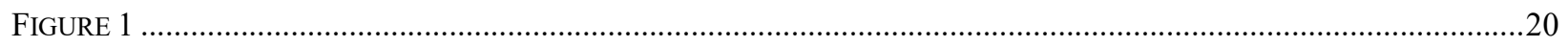

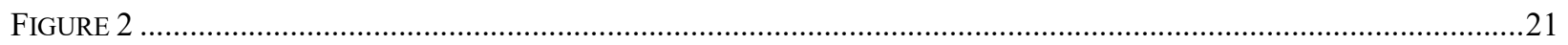

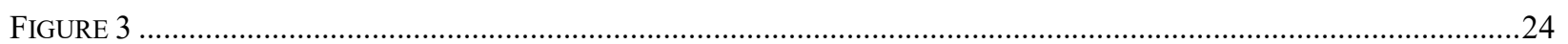

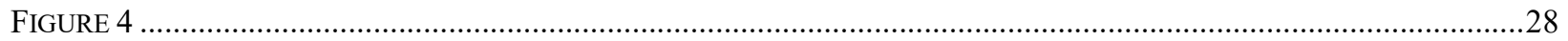

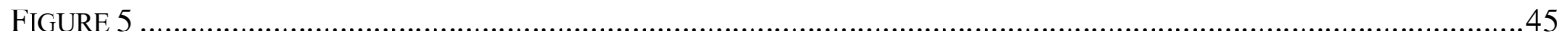

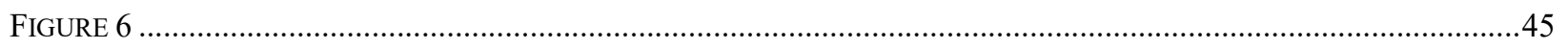

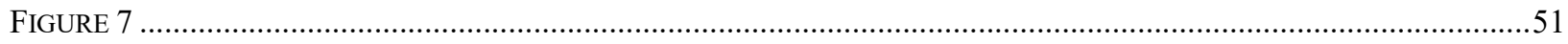

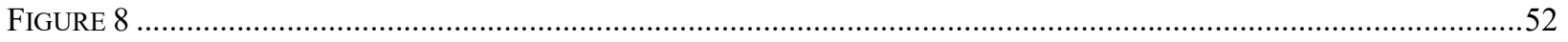

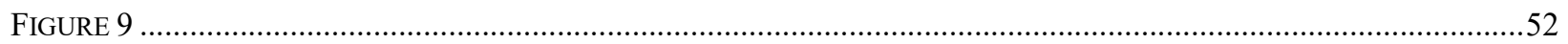

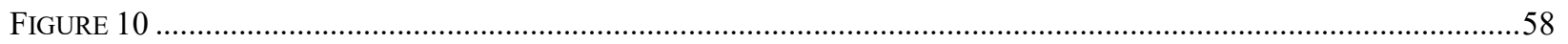

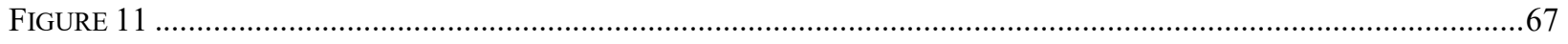

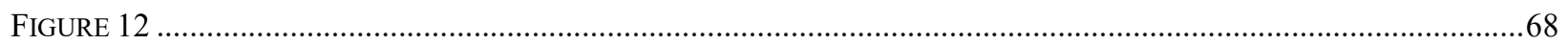

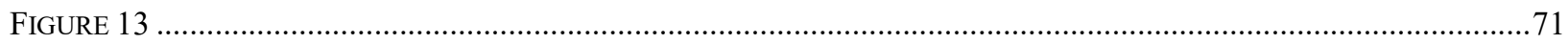

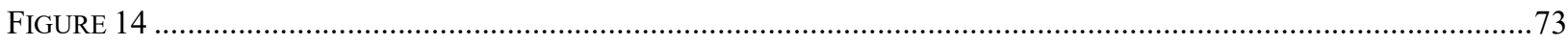

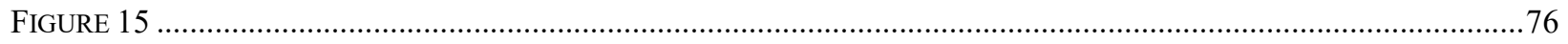

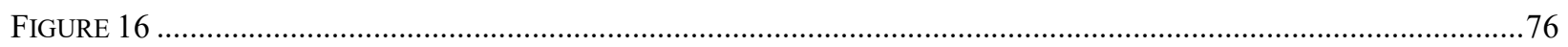

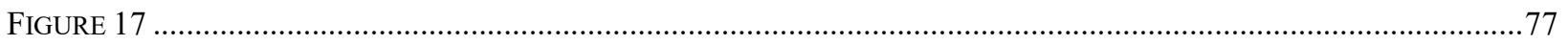

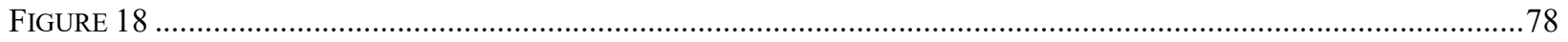


Spatial reasoning is required for many topics in undergraduate mathematics. Some of these topics are fairly obvious, such as those related to geometry, while others are not. As an example, calculus topics such as solids of revolution and curve sketching require students to use some spatial skills to make informed decisions and produce accurate figures. It has been shown that spatial abilities and mathematical abilities are related (Uttal \& Cohen, 2012; Xie et al., 2020; Young et al., 2018), so one may infer that measuring a student's spatial abilities may provide insight into the potential for success in mathematics, and improving upon spatial abilities may lead to increased success in mathematics.

Unlike topics such as algebra and trigonometry, there are few interventions that assess students' spatial skills and provide remediation. This means many students struggle to perform tasks that require a certain level of spatial skills, which leads to a lack of understanding. For example, students with under-developed spatial abilities may struggle to understand how to rotate a two-dimensional graph in order to form a three-dimensional solid. This is a fundamental skill necessary for computing volumes of solids of revolution. If a student cannot properly rotate the two-dimensional graph, he or she will struggle to understand how the volume of the threedimensional object is calculated.

Spatial skills are not only necessary for mathematics, however. Understanding molecular structure in chemistry, for instance, requires spatial skills (Carlisle et al., 2015). The same can be said for understanding motion in physics. Because of this, some focus should be placed on understanding what level of spatial skills students entering undergraduate programs possess and how we can help undergraduate students improve their spatial skills.

Haptic technology is something that is new to most students. Because of this, it is a novel approach to improving spatial skills. Students are more likely to remain engaged due to their 
unfamiliarity with the technology, which will help with better retention of the learned skills. Haptic environments also provide a level of flexibility; instead of being restricted to a few physical models of simple three-dimensional shapes because they are the only ones that are available, the possibilities become nearly endless (as long as the shape can be built in the computer-based environment). This allows for much practice with various objects, which should allow for more improvement in spatial skills.

The purpose of this dissertation is two-fold: first, to determine what level of spatial abilities students possess upon entering an undergraduate calculus course, and second, to determine whether haptic feedback will enhance an intervention for improving spatial skills. Determining students' entry-level abilities will help instructors understand what challenges their students will face regarding problems that require spatial skills. Determining whether haptic feedback is a valid tool for improving spatial skills will inform on whether a haptic-based remediation is something that could be utilized to help undergraduate students improve their spatial skills in a stand-alone environment (outside the mathematics classroom). 


\section{Definitions and Literature Review}

Two key components make up the background for this research: spatial skills (or abilities) and haptics. Presented for each component are the definitions of key terms and an examination of related literature.

\section{Spatial Skills}

Skill is "the ability to do something that comes from training, experience, or practice" (Merriam-Webster, n.d.) and ability is "the power or skill to do something" (Merriam-Webster, n.d.). By these definitions, skills and abilities are synonymous. This is also evidenced in the literature, as many authors interchange the two terms spatial skill and special ability. In general, spatial skills (or spatial abilities) are skills involving objects in space. However, this definition does not provide a complete picture. To better explain, we will examine the different definitions in the literature.

\section{Definitions}

According to the National Research Council (2012), spatial ability is "the ability to mentally manipulate two-and three-dimensional objects" (p. 98) and can be small- or large-scale. Small-scale abilities deal with the manipulation of objects, while large-scale abilities involve routes and environmental layouts. Albert and Golledge (1999) add that spatial abilities "involve the retention, manipulation, and recognition of spatial stimuli” (p. 10). Although many authors seem to agree with this overall definition (Kersh et al., 2008; Kurtulus, 2013; Lawrence, 2011; McGee, 1979; Xie et al., 2020), there is some disagreement as to the number and kinds of spatial abilities.

Thurstone (1969) claimed three distinct abilities: spatial orientation, spatial visualization, and spatial relation. Lohman agrees with this idea, but adds the possibility of other minor factors 
(as cited in Pittalis \& Christou, 2010). Some of these minor factors may be included in the list of abilities Kimura (1999) provides, which adds spatial location memory, targeting, and disembedding to the initial three abilities. McGee (1979), on the other hand, considers only spatial orientation and spatial visualization to be factors of spatial ability.

Spatial orientation is "the ability to imagine how a visual stimulus or configuration looks from a different perspective" (Albert \& Golledge, 1999, p. 10). This correlates with the perspective-taking factor proposed by Hegarty and Waller (2004). McGee (1979) further says that spatial orientation involves "the aptitude to remain unconfused by the changing orientations in which a spatial configuration may be presented" (p. 897) and is important for tasks such as map reading and sense of direction.

McGee (1979) describes spatial visualization as "the ability to mentally rotate, manipulate, and twist two- and three-dimensional stimulus objects" (p. 896). This skill is considered to be a factor of success in college mathematics (McGee, 1979). Spatial visualization differs from spatial orientation in that orientation tasks require a change in the position of the person, while visualization tasks require a change in the object. Determining whether someone has used spatial visualization or spatial orientation when completing a task can be difficult, as it depends on the method used to perform the task. For example, a student may be shown an object and asked to think about what the view looks like from a different perspective. If the student uses spatial visualization to complete the task, he or she will imagine rotating the object until the proper perspective is achieved; in contrast, if the student uses spatial orientation to solve the task, he or she will imagine moving him or herself to the location that gives the proper perspective.

Pittalis and Christou (2010) add four categories of abilities specific to mathematics into which visualization and orientation can be broken: representing objects, structuring, 
measurement, and mathematical properties. Representing objects is defined as manipulating forms of objects and constructing models. Structuring is defined as constructing and manipulating partitions of objects. Measurement is defined as calculating and estimating. Finally, mathematical properties is defined as realizing, identifying, and comparing structural elements.

\section{Literature Review on Training for Spatial Skills}

Improving students' spatial skills is important because spatial ability has been linked to mathematical ability. A meta-analysis of 73 studies ranging in date from 2008 to 2018 performed by Xie et al. (2020) found that there is a positive and significant correlation between spatial ability and mathematical ability. Young et al. (2018) agree with this finding, but note there is a definite gap when it comes to understanding how spatial skills and mathematical skills are connected. These authors encourage the training of spatial skills to promote success in mathematics.

An examination of literature related to the training of spatial skills gives evidence that spatial skills can be improved by undergoing training. A meta-analysis of spatial skill training studies completed by Uttal and Cohen (2012) analyzed 206 articles, ranging in date from 1984 to 2009. Through this analysis, the authors found that training led to an improvement of one-half standard deviation, supports the authors' conclusion that "transfer of spatial training is possible" (Uttal \& Cohen, 2012, p. 174).

Success in spatial skills training has continued since the conclusion of the meta-analysis. Several studies have been performed with engineering students, as spatial abilities are considered an essential part of success in engineering fields. Studies performed by Martin-Gutiérrez et al. (2010), Martin-Dorta et al. (2011), and Sorby et al. (2013) each demonstrated improvement in engineering students' spatial skills following the completion of a spatial training course. The 
latter study also found a correlation between the increase in spatial skills and success in a calculus course.

Spatial training has also been investigated in mathematics. An initial study performed by Taylor and Hutton (2013) examined the use of origami and pop-up paper engineering as a spatial skills training tool in elementary students. This study was expanded by Burte et al. (2017) to include investigation of whether the training also impacted mathematics abilities. Both studies showed improvements in spatial skills among the students, and the follow-up study showed improvement among all students on real-world math problems and among older students on problems involving visual representations and spatial thinking. Lowrie et al. (2019) also found success in training elementary mathematics students by instantiating an intervention program over a three-week period that focused on spatial visualization problems. This study demonstrated significantly greater gains in spatial skills and mathematical performance in the experimental group than in the control group.

Finally, spatial training has been performed in other STEM fields. Carlisle et al. (2015) examined the effect of brief interventions performed over the course of a semester in undergraduate general chemistry students. While the results demonstrated significant differences between the experimental and control groups, the authors noted that there was evidence that more training was needed. Applebee et al. (2021) found that students who participated in a weeklong spatial skills bootcamp not only improved their spatial skills, but also performed significantly better in an organic chemistry course than their matched-pair counterparts. The pairs of students did not, however, perform significantly different in the physics course.

Lee and Wong (2014) examined the difference between high school students using virtual reality to learn about the anatomy of a frog and the more traditional classroom method of 
PowerPoint slides. The results of the experiment showed that the virtual reality environment promoted a larger increase in scores from pre-test to post-test than the traditional learning environment. Furthermore, the performance of students with lower spatial abilities was affected more by the learning mode than the performance of students with higher spatial abilities.

\section{Literature Review on Definite Integrals}

Many topics covered in calculus require the use of spatial skills: graphing, curve sketching, tangent lines, transformations of functions, and more. This study uses the topic of volumes of solids of revolution to both test and train students' spatial skills. According to Jones (2015b), volumes of solids of revolution is just one of many topics that require the use of Riemann sums in order to construct a robust understanding of the concept. This idea comes from the consideration of the volume of the solid of revolution as the sum of the volumes represented by its cross-sections with infinitesimally small width (represented by $d x$ or $d y$ ). That being said, it is important to consider research pertaining to students' conceptions of the definite integral.

It has been shown by several authors that in order for students to be successful with realworld applications of definite integrals, they must view the definite integral as a sum of infinitely many pieces (Jones, 2015a, 2015b; Meredith \& Marrongelle, 2008; Sealey, 2006, 2014;

Thompson \& Silverman, 2008). Thompson and Silverman (2008) note that viewing the definite integral as the accumulation of quantities that are formed via multiplication is essential for students to understand that the "area under a curve" may represent something other than area, as it does in volumes of solids of revolution.

Jones (2015a) refers to this idea as the "multiplicatively-based summation conception" (p. 24), as the quantity being accumulated is found via multiplication and the results must be summed to get the value of the integral. Through interviews with students, Jones (2015a) and 
Meredith and Marrongelle (2008) both determined that this view of definite integrals was highly productive when it came to the students making sense of the problems.

Unfortunately, many students do not view the definite integral in this manner. A later study by Jones (2015b) determined that "around half of all successful first-semester calculus students might not draw on any summation conception as a basis for explaining the meaning of these types of definite integral" (p. 731). This idea is supported by research on the framework created by Sealey (2014). Through the examination of student processes in solving application problems she found that the students struggled most with the product layer of the framework, which involves conceptualizing the quantity being accumulated. With this being the case, students are likely to struggle with volumes of solids of revolution.

\section{Haptics}

Haptic is "relating to or based on the sense of touch" (Merriam-Webster, n.d.). Thus, haptic feedback is feedback observed through the sense of touch. For example, the vibration feature on a cell phone is considered haptic feedback. Haptic feedback is also common in some gaming systems, through vibrating game controllers. When used with a three-dimensional computer-based environment, the combination of visual and haptic feedback allows the user to "feel" an object that is on the screen. Haptic devices are often classified by their degrees of freedom. Degrees of freedom refers to the number of positional arguments tracked by the device. The most common devices have three degrees of freedom, tracking location along the $x$-, $y$-, and $z$ - axes, or six degrees of freedom, which adds in roll, pitch, and yaw. 


\section{Literature Review on Haptic Feedback used in Education}

There are many studies involving the educational uses of haptics. Most commonly, these studies fall into one of three categories - teaching science concepts, performing medical training, or studies with subjects who have visual impairments.

The use of haptics to learn science concepts is intuitive, as haptic effects can demonstrate the physical properties related to the concepts. Studies have been done with middle school students to learn biology concepts such as passive transfer (Minogue et al., 2006) and animal cell structure, including organelle identification (Jones et al., 2006). Although the studies done by Minogue et al. (2006) and Jones et al. (2006) both showed gains in student performance, there was no evidence that the gains were attributed specifically to the haptic feedback.

Studies in chemistry had similar results (Bivall et al., 2011; Persson et al., 2007), in that gains were made from the pre-test to the post-test, but significant differences did not exist between the experimental and control groups on the post-test. Through clinical interviews, however, Persson et al. (2007) found evidence of more explanations involving force, which implied that "haptics did successfully convey the importance of forces" (p. 177). Similarly, Bivall et al. (2011) found that the haptics condition was able to prevent the occurrence of a common misconception related to forces, and that "reasoning in the haptics group was generally deeper and elaborated more on energy and stability ideas" (p. 715).

Physics education is a rich area of haptics study because of the nature of the concepts.

Physics deals with force and motion and therefore is a prime content area for haptic applications. Hamza-Lup and Adams (2008) found that students who performed activities involving applications of hydraulics with haptic feedback had a better understanding of Pascal's principle than the students who performed the activities without haptic feedback. Gorlewicz (2013) 
analyzed three years of data from labs using the haptic paddle (one degree of freedom) to learn about various physics concepts. The data analysis showed a significant difference in cumulative pre-test and post-test scores, indicating learning occurred overall. The scores were also significantly higher when the quiz was taken after the lab (as opposed to the beginning of the lab or after the pre-lab lecture), indicating the lab was indeed crucial to the learning process.

Medical training is a common application for haptics due to the possibility of performing an unlimited number of trials without the danger presented with human subjects. There is a vast amount of recent literature pertaining to medical training. One example is the study performed by Chellali et al. (2012) which examined the training of medical students in how to plan and perform biopsies. Students who trained using haptic feedback were faster, used fewer landmarks, had less contact with organs, had fewer organ penetrations, had shorter insertion paths, and missed less frequently than students who did not. These students also had a significantly shorter insertion time.

Studies have also been done specifically to determine whether haptics-based applications can promote learning in students with visual impairments. A total of 20 haptics-based applications were developed using the framework described by Darrah et al. (2014) to teach various middle school mathematics and science topics (Darrah M. A., 2013; Murphy \& Darrah, 2015). After undergoing rigorous testing on design and usability, testing was done to determine whether the applications promoted learning in students with visual impairments (Darrah M. A., 2013; Murphy \& Darrah, 2015). Each application tested showed significant learning gains in student understanding.

Toennies et al. (2011) performed two experiments to evaluate whether an application for Cartesian planes, shapes, lines, and slopes could be useful for students with visual impairments. 
For these experiments, different combinations of auditory and haptic feedback were used in order to determine which feedback resulted in the most success for performing the tasks. Neither experiment showed a significant difference between the different types of feedback. Shimomura et al. (2013) represented several geometrical shapes in different ways to determine whether haptics can be used to conceptualize three-dimensional geometry information. This study found that orientation and excess information (i.e., the ability to explore the space freely) provided the most difficulties when attempting to identify three-dimensional objects.

\section{Conclusion}

As has been shown in several studies, spatial skills can be increased through spatial training, whether that training be a stand-alone course or instruction that has been integrated into existing coursework. A link between spatial skills and mathematical ability has also been found, indicating that an improvement in spatial skills may result in an improvement in mathematical performance. There is little research, however, on the training of spatial skills in mathematics, and the studies that do focus on mathematics are generally performed with students at an elementary school level.

Similarly, there is much research on using haptics to teach concepts in science, but haptics use in mathematics is fairly limited. The instances found in which haptics is used in a mathematics setting focus on teaching students with visual impairments or validating a haptics tool for use with students with visual impairments. No studies were identified that focused on undergraduate mathematics or teaching spatial skills in a mathematical setting, and no studies were found that specifically examine the effects haptics may have on spatial skills.

The goal of this research was to begin to fill the gaps identified above by examining spatial skills training in an undergraduate mathematics setting, using haptics as a tool for 
training. Concepts of volumes of solids of revolution were used for the testing and training of spatial skills as the topic requires the use of spatial skills, lends itself to the use of haptics in training, and is related to an essential skill in calculus, the definite integral. 


\section{Research Questions}

The goal of the dissertation research was to determine what spatial abilities students possess when entering calculus, whether those spatial abilities that are under-developed (if any) can be remediated, and if so, whether haptics can play a role in developing those spatial abilities. These concepts were broken down into four research questions to be answer by the dissertation experiment. The following sections present the questions answered by the dissertation research and how the research allowed each question to be answered. Key artifacts used to answer the research questions include the Santa Barbara Solids Test (SBST), given as a pre-test and posttest to all students of a two-semester Calculus I course at West Virginia University (WVU), and pre- and post-interviews conducted with a subset of the students who scored below-average on the pre-test, split into matched pairs.

\section{Research Question 1: What Spatial Skills Related to Cross-Sections Do Students Entering}

\section{Calculus Possess?}

This question was answered by examining the results of the SBST pre-test and the preinterviews. Because the pre-test was given at the beginning of the semester to all students in the course, the results reflect students' abilities upon entering the course. The large population (306 students) also means the results can be considered typical across all students entering this version of Calculus I at WVU. The SBST is designed to test spatial visualization by asking the students to determine the shape of the cross-section. It also tests spatial orientation, by requiring students differentiate between the egocentric answer, in which the student does not properly orient him or herself with the cross-section, and the proper answer. The pre-test results were analyzed to determine whether students possess spatial visualization and orientation skills when they enter calculus. 
The pre-interviews primarily examined the following spatial skills related to crosssections: spatial orientation, spatial visualization - representing objects, and spatial visualization - structuring. Spatial orientation was further tested by questions taken from the SBST as well as cross-section questions with real objects. Spatial visualization - representing objects and spatial visualization - structuring were tested by asking students to determine how an object should be sliced (i.e. what cutting plane should be used) in order for the sum of the areas of the resulting cross-sections to be easily calculated, assuming any necessary measurements could be taken. For these types of questions, the student needed to manipulate the three-dimensional model in multiple ways and construct multiple two-dimensional models (cross-sections). The student also needed to consider how the resulting cross-sections fit together to form the three-dimensional object.

Because the interview students scored below average on the SBST pre-test, the results are limited to representing only students whose spatial orientation skills are under-developed. These results are important as they present a robust depiction of the students with lower spatial orientation abilities. A correlation between the results of the pre-test and the results of the preinterview would mean extrapolation to the entire population may be possible.

\section{Research Question 2: What Difference in Performance Does the Haptic Group Exhibit Over the Non-Haptic Group in the Post-Interview?}

The purpose of this research question was to determine whether the addition of haptic feedback promoted an increase in spatial skills. The most basic way to do this was to compare the scores of the post-interview activities across the two matched pair groups. It was the hope that this would give evidence of which group performed better and which format - visual or visual with haptic feedback - had a larger impact on spatial abilities. 
To get a more informed picture of the performance of the groups, the thought processes of the students during the post-interviews were analyzed to see how the students used the various spatial skills to come to conclusions. Then, the post-interviews were compared to the preinterviews in an attempt to see whether adding haptics to the intervention made a difference in student learning or the processes the students used to perform the interview tasks.

\section{Research Question 3: How Do the Performance and Explanations Differ Among the Interview Population From the Pre-Interview to the Post-Interview?}

This question was answered by comparing the pre-interview and post-interview for each student in the Interview Population. At the most basic level, the interviews were compared to determine whether student performance improved from the pre-interview to the post-interview. Student explanations were also examined to determine whether the student had a better understanding of the material. The analysis of the explanations was important because students can guess the correct answer without actually understanding the content, and students can also have a good understanding of the content, but make a simple mistake. These explanations were also analyzed to determine whether the language used changed in any way. In particular, a focus was placed on language related to spatial skills and haptic thinking.

\section{Research Question 4: How Does the Difference in the Pre/Post Spatial Skills Test Given at the Beginning and the End of the Semester Differ Among the Groups?}

Through the course of the study, the students who participated in the research were referred to as a part of six groups (see the Research Methods section for details on the groups). To answer this question, the improvements in each of the groups were compared. The main goal was to discover which group made the most improvement in order to determine: 1) whether spatial skills improved without the use of the intervention, 2) whether students who participated 
in the intervention showed more improvement in spatial skills than the students who did not participate in the intervention, and 3) whether the students who received haptic feedback in the intervention showed more improvement than the students who did not receive haptic feedback. It has been shown that spatial skills are necessary for students to understand integrals and volumes of solids of revolution (Engelke et al., 2016), so this information was critical in making an argument for spatial skill remediation in calculus students. 


\section{Theoretical Perspective}

\section{Constructivism}

Spatial reasoning is not first introduced in calculus, nor is it restricted to mathematics; we use spatial reasoning every day from the time we are small children, though not always in a mathematical sense. Because of this, students enter calculus with a variety of spatial abilities and conceptions based on their past experiences. In order for students to be successful in performing spatial tasks in calculus, they must be able to build on a solid foundation of spatial reasoning skills that have been previously developed. This leads naturally to examining students' spatial abilities through the lens of Constructivism.

Constructivism is based on the idea that knowledge is constructed from "our perceptions and experiences, which are themselves mediated through our previous knowledge" (Simon, 1995, p. 115). According to Schoenfeld (1987), this perspective embraces the notion that "we build our own interpretive frameworks for making sense of the world, and we then see the world in light of these frameworks" (p. 22). This defines the learner as the active constructor of knowledge (Prawat \& Floden, 1994). Knowledge is built by the learner actively recognizing and solving problems, asking questions, and creating new problems (Dubinsky, 1991).

This perspective is in contrast to the idea that students arrive without any previous knowledge of concepts. Simon (1995) notes that in the process of schema creation, there is no way to know if it is correct; one can only know if it is consistent with the experiential world on which it was built. The result of this is that students may construct interpretations that are consistent with the subject matter, but turn out to be incorrect (Schoenfeld, 1987).

Piaget describes this using the terms assimilation and accommodation. Assimilation is "the process whereby an action is actively reproduced and comes to incorporate new objects into 
itself' (Piaget, 1970, p. 63). This means the learner develops a new understanding of the information in order for it to fit into the existing structure. Accommodation, on the other hand, is "the process whereby the schemes of assimilation themselves become modified in being applied to a diversity of objects" (Piaget, 1970, p. 63). This occurs when the learner modifies the already formed structures so the new information is consistent. Thus, instead of arriving as a "blank slate", students actually arrive with notions as to what is going on, many of which may be incorrect. It is up to the instructor to push the students to enter an adaptive process by presenting them with experiences that differ from the incorrect constructs.

Based on these ideas, spatial abilities can be improved, but they are not explicitly taught in the manner that addition and subtraction are. There are no formulas for spatial abilities, nor are there step by step instructions to follow. Spatial abilities must be constructed individually and are based on experiences. For example, a sighted person uses vision to navigate and determine distances between objects. A non-sighted person, however, uses the sense of touch to determine the same information. If both were asked to perform the same spatial task, the advantage would go to whichever is more familiar with that mode: the sighted person would be at a clear advantage in a visual spatial task; the non-sighted person would be at a clear advantage in a tactile spatial task.

This advantage, however, does not have to exist forever. By being exposed to more tactile spatial tasks, the sighted person can develop the ability to perform the necessary skills. Similarly, though a non-sighted person will not be able to see as a sighted person would, he or she can develop techniques to assist in the performing of the task. By gaining experience in the unfamiliar realm, knowledge of performing spatially in that realm is constructed, and the necessary abilities are developed. 


\section{Spatial Skills Framework}

The goal, then, is to promote the development of spatial abilities by exposing students to a variety of spatial activities. To assist with this, Engelke et al. (2016) developed a Spatial Skills Framework to determine what spatial skills are most common in calculus by breaking down various types of problems encountered in the course. This would allow remediation to focus on the skills that are most necessary for success. In this framework, the authors combine the four spatial categories introduced by Pittalis and Christou (2010) (representing objects, structuring, measurement, and mathematical properties) with the two spatial abilities (orientation and visualization), and further break skills down based on dimension (two-dimensional and threedimensional). Definitions of the spatial categories and abilities can be found in the Definition and Literature Review section. This results in $16(4 \times 2 \times 2)$ potential categorizations for skills required to complete a spatially related calculus problem.

To use the framework, one must break a mathematics problem down into individual steps and determine what spatial skills are used at each step, keeping in mind that any given step may use multiple spatial skills. As an example, the authors broke down a common calculus problem: computing volumes of solids of revolution (see Figure 1 and Figure 2, taken from Engelke et al., 2016). Students must draw on both two-dimensional and three-dimensional spatial skills in order to properly rotate a two-dimensional region to form a three-dimensional solid, then determine how to slice that solid and properly calculate its volume. 


\section{Figure 1}

The Steps Necessary to Compute the Volume of a Solid of Revolution

STEP 1:

Graph function: $f(x)=-\frac{1}{4}(x-2)^{3}+2$.

STEP 3:

Reflect the region about $y=0$.

STEP 5:

Determine which way to slice:

horizontally or vertically?

STEP 7:

Draw radius and measure.

Determine limits of integration (0 to 4$)$.
STEP 2:

Create $2 \mathrm{D}$ region with $f(x), x=0, y=0$.

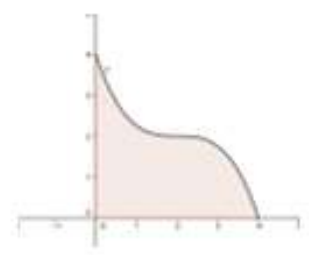

STEP 4:

Complete the $3 \mathrm{D}$ shape by rotating.

STEP 6:

Position 3D disks.

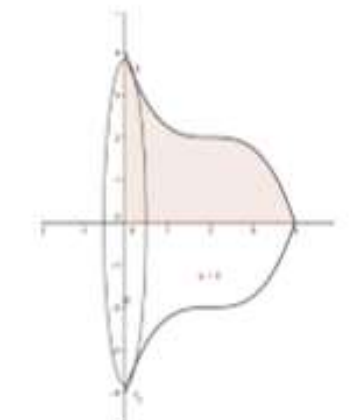

STEP 8:

Access volume of a cylinder formula: $V=\pi r^{2} h$

\section{STEP 9:}

Set up integral with boundaries.

$\int_{0}^{4} \pi(f(x))^{2} d x=\int_{0}^{0} \pi\left(-\frac{1}{4}(x-2)^{3}+2\right)^{2} d x$

Note: From "A framework for examining the 2-D and 3-D spatial skills needed for calculus," by

N. Engelke et al., 2016 Proceedings of the 19th Annual Conference on Research in

Undergraduate Mathematics Education, pp. 737-738. 


\section{Figure 2}

Categorizing the Spatial Skills Necessary to Compute the Volume of a Solid of Revolution

\begin{tabular}{|c|c|c|c|c|c|c|c|c|}
\hline & \multicolumn{4}{|c|}{$2-D$} & \multicolumn{4}{|c|}{$3-D$} \\
\hline & 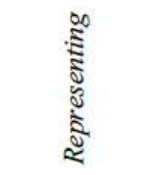 & 䒿 & 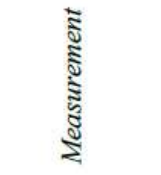 & $\frac{\dddot{y}}{2}$ & 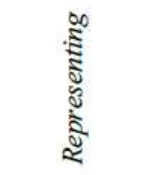 & 宽 & 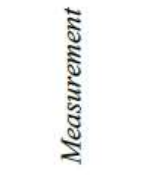 & 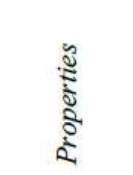 \\
\hline Visualization & $\begin{array}{l}\text { Step 1: } \\
\text { Draw 2D } \\
\text { graph. } \\
\text { Step 2: } \\
\text { Identify } \\
\text { region to be } \\
\text { rotated. }\end{array}$ & & & $\begin{array}{l}\text { Step 7: } \\
\text { Determine } \\
\text { the radius } \\
\text { of a slice. }\end{array}$ & $\begin{array}{l}\text { Step 4: } \\
\text { Imagine the } \\
\text { 2D region } \\
\text { being } \\
\text { rotated and } \\
\text { the resulting } \\
\text { 3D shape. }\end{array}$ & $\begin{array}{l}\text { Step 6: } \\
\text { Imagine } \\
\text { slices } \\
\text { filling up } \\
\text { the figure. }\end{array}$ & & \\
\hline Orientation & $\begin{array}{l}\text { Step 3: } \\
\text { Identify the } \\
\text { axis of } \\
\text { rotation. }\end{array}$ & & $\begin{array}{l}\text { Step 7: } \\
\text { Determine } \\
\text { the radius of } \\
\text { a slice. }\end{array}$ & $\begin{array}{l}\text { Step 7: } \\
\text { Determine } \\
\text { the limits } \\
\text { of } \\
\text { integration. }\end{array}$ & $\begin{array}{l}\text { Step } 5: \\
\text { Determine } \\
\text { which way } \\
\text { to slice- } \\
\text { horizontally } \\
\text { or } \\
\text { vertically? }\end{array}$ & & $\begin{array}{l}\text { Step 8: } \\
\text { Determine } \\
\text { the volume } \\
\text { of a slice. } \\
\text { Step 9: Set } \\
\text { up and } \\
\text { evaluate the } \\
\text { integral. }\end{array}$ & $\begin{array}{l}\text { Step } 8: \\
\text { Identify } \\
\text { the } \\
\text { volume of } \\
\text { a cylinder } \\
\text { formula. }\end{array}$ \\
\hline
\end{tabular}

Note: From "A framework for examining the 2-D and 3-D spatial skills needed for calculus," by N. Engelke et al., 2016 Proceedings of the 19th Annual Conference on Research in Undergraduate Mathematics Education, p. 738.

Representing objects is put to use when a student attempts to draw a cross section of a three-dimensional object. Structuring is used when a student must consider the cross sections that result from slicing an object an infinite number of times using parallel cutting planes.

Measurement is involved in performing the actual calculation of the area of a cross-section.

Finally, mathematical properties involves structural elements such as limits of integration. 
Initially, the framework is used to represent the most common method a student would follow while working through a particular type of problem. In a similar manner, the framework can be used to examine a student's actual thought process while working through a specific problem. By examining the discrepancies between the student's actual process and the ideal process, we can begin to understand where difficulties arise and focus on those individual spatial abilities.

\section{Theoretical Perspective for Remediation of Spatial Skills}

A combination of these two ideas can be used when considering undergraduate mathematics students and their spatial reasoning abilities. Constructivism can be used to explain how spatial skills develop and as a basis for creating remedial programs. The Spatial Skills Framework can be used to determine what skills a student possesses or lacks and to identify difficulties he or she may have in the process of solving a particular mathematics problem that requires spatial reasoning. 


\section{Spatial Reasoning Tests}

The following spatial reasoning tests are referred to throughout this document. The tests are presented separately here in order to provide in-depth descriptions that can be easily referred to as necessary.

\section{Santa Barbara Solids Test (See Appendix A: Santa Barbara Solids Test)}

The Santa Barbara Solids Test (SBST) was developed by Cheryl A. Cohen and Mary Hegarty (Cohen \& Hegarty, 2012). The test was designed as a means of determining what difficulties undergraduate students have in determining cross-sections of three-dimensional objects. The test is comprised of 30 multiple-choice questions. Each question gives the student a picture of a figure transected by a cutting plane. The student's task is to determine the shape of the cross section that is a result of cutting the figure with the displayed cutting plane.

Four answer choices are given for each question: the correct answer, the egocentric distracter, the combination distracter, and the alternate distracter. The egocentric distracter is the correct shape of the cross-section presented at an incorrect perspective (the test requires the student to act as if they are looking at the cross-section head-on). This means the student has identified the correct shape, but is not able to view it with the correct orientation. The combination distracter is a combination of two possible cross-sections. The alternate distracter is a valid cross-section of the figure that is found using a different cutting plane. In Figure 3, answer (c) is the correct answer, answer (b) is the egocentric distractor, answer (a) is the combination distractor, and answer $(\mathrm{d})$ is the alternate distractor. 


\section{Figure 3}

A Sample Problem From the SBST

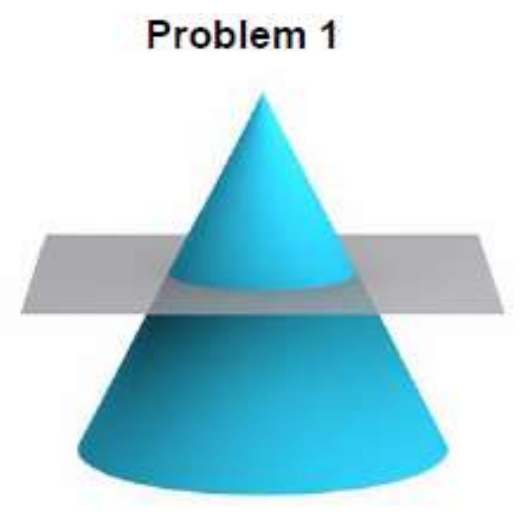

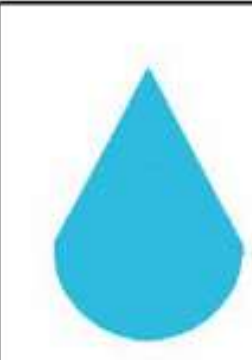

(a)

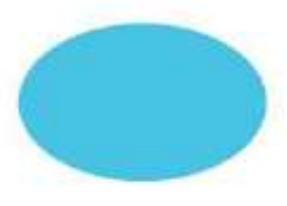

(b)

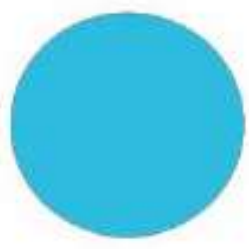

(c)

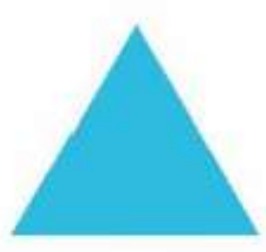

(d)

Note: (c) is the correct answer, (b) is the egocentric distractor, (a) is the combination distractor, and (d) is the alternate distractor.

Three types of figures are used in the test: simple figures, which are geometric solids such as cones and cylinders; joined figures, which are simple figures that are attached at their edges; and embedded figures, which consist of one simple figure embedded in another (Cohen \& Hegarty 2012). Each type of figure comprises one-third of the test questions. There are also two 
types of cutting planes in the test: orthogonal planes (horizontal or vertical), and oblique planes.

Each type of cutting plane comprises one-half of the test questions.

Spatial Reasoning Aptitude Test - Hard (See Appendix B: Spatial Reasoning Aptitude Test - Hard)

The Fibonicci website (www.fibonicci.com) contains several aptitude tests including Numerical Reasoning, Logical Reasoning, Verbal Reasoning, Spatial Reasoning, and NonVerbal Reasoning tests (Fiboni V.O.F., n.d.). The tests provided by the website are to be used as training exercises to prepare for psychometric tests, pre-employment tests, and admission tests that utilize aptitude tests to help discriminate between applicants.

The Spatial Reasoning Aptitude Test - Hard consists of 12 multiple choice questions (Fiboni V.O.F., n.d.). Each question displays a two-dimensional net of a three-dimensional figure accompanied by four three-dimensional figures as answer choices. The student must choose which three-dimensional figure is formed by folding the displayed two-dimensional net. Of the 12 questions, 9 present nets that fold into complex objects. Two questions present simpler objects, but the student must pay careful attention to the coloring of the sides. One question contains a net that folds into an open shape.

\section{Revised Purdue Spatial Visualization Tests: Visualization of Rotations (See Appendix C: Revised Purdue Spatial Visualization Test: Rotations)}

The Purdue Spatial Visualization Tests: Visualization of Rotations (PSVT:R) was developed by Roland B. Guay in 1976 (Bodner \& Guay, 1997). The test is comprised of 30 multiple choice questions in which the student is presented an example figure and its result after a rotation, then must determine which choice represents a second figure under the same rotation. As opposed to many other rotations tests which contain simple block figures, the figures in this 
test have inclined, oblique, and curved surfaces, thus making the mental rotation of the objects more difficult. The test is designed to be completed in 20 minutes.

The PSVT:R was revised by So Yoon Yoon in 2011 to correct figural errors found by Jianping Yue (Yoon, 2011). These errors included missing and extra features in both the example figures and the answer choices. The necessary revisions were determined by first drawing each figure using a graphic design program and rotating the figure to compare the result to the objects on the test. Comparisons were also done to three-dimensional figures constructed of clay and wood. Finally, a panel of qualified doctoral students examined the figures to confirm the errors and necessary revisions. 


\section{Pilot Studies}

Two smaller pilot studies were conducted in order to provide a foundation for a larger scale intervention design and research study. Each pilot study presented different challenges and insights to the researcher, which are evidenced in the primary research design. Participants of the pilot studies are identified by pseudonyms.

\section{Pilot Study 1}

The first pilot study was conducted in the Fall of 2013. The purpose of this pilot study was to determine how a student's spatial reasoning skills affect his or her ability to set up the appropriate integral representing the volume of a solid of revolution.

\section{Methods}

Interviews were conducted with three students enrolled in a second semester Calculus course. Each interview consisted of four parts: warm-up questions, spatial reasoning, physical volumes, and solids of revolution. The goal of the warm-up questions was to accustom the student to the interview setting and gain insight into his or her background relevant to the interview material. Each student was asked about his or her mathematical background, the types of toys he or she played with as a child, whether he or she liked to do puzzles, and whether he or she participated in sports as a child.

The spatial reasoning section consisted of a two-section spatial reasoning test, each section consisting of 3-4 multiple choice problems (time permitting). Section one consisted of problems taken from the Santa Barbara Solids Test, while section two consisted of problems taken from the Spatial Reasoning Test - Hard (see Figure 4). Students were asked to justify their answer choice as well as refute the remaining answer choices. In times of uncertainty, students were instructed to choose the option they thought was most likely to be correct. 


\section{Figure 4}

The Spatial Reasoning Test for Pilot Study 1
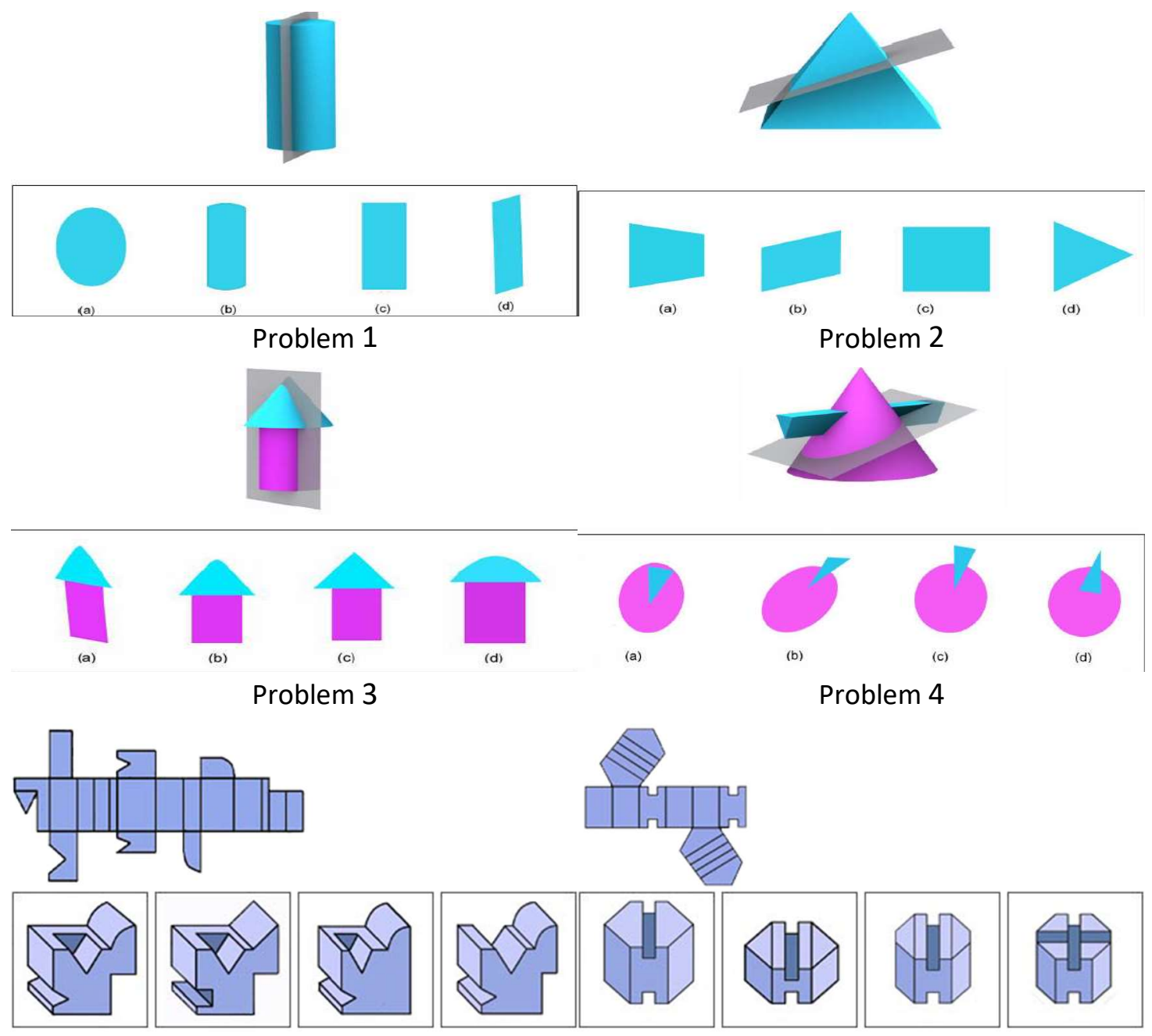

Problem 5

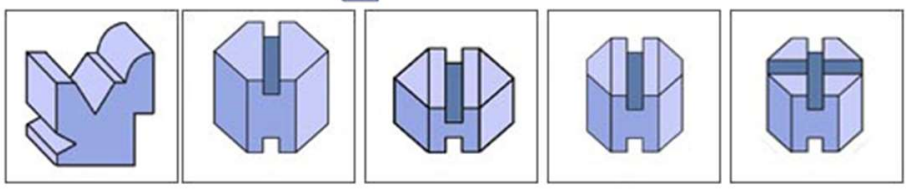

Problem 6
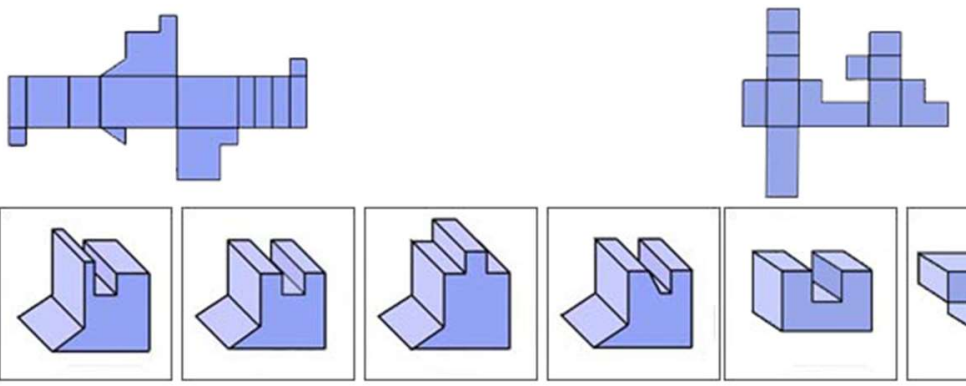

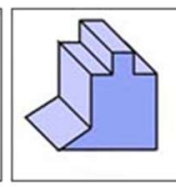

Problem 7
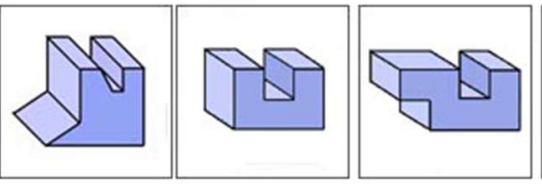

Problem 8

Note. The first four problems are taken from the Santa Barbara Solids Test. The last four problems are taken from the Spatial Reasoning Test - Hard. 
The physical volumes section of the interview asked the students to describe how they would estimate the volume of three different foods: a donut, a banana, and a butternut squash. Students were urged not to be overly concerned with formulas, but to give a procedure for finding the volume. Students were also told to assume they had the ability to take any necessary measurements and to cut the object however they desired. Finally, the solids of revolution section presented the student with 2-3 (time permitting) solids of revolution for which to compute the volume. These problems can be seen in Appendix D: Pilot Study 1 Solids of Revolution Problems. Volumes were covered in class prior to the interviews, so students were expected to be familiar with the material in this portion of the interview. Similar to the physical volumes portion, students were urged to focus not on obtaining a numerical answer, but on presenting an integral that would lead to the precise volume. Students were asked to explain what each part of the resultant integral represented and to justify their choice of method.

The only significant changes made to the interview protocol throughout this process were to correct errors found in the solids of revolution portion of the interview. The initial interview exposed some minor errors in the phrasing of the problems that required the student to ask for clarification. To remedy this, the bounds listed in the problems were adjusted to better describe the volume. There was also a small typographical error that required attention.

\section{Results}

Results of the spatial reasoning test, physical volumes, and solids of revolution portions of the interviews were analyzed.

Spatial Reasoning Test. Each student correctly answered four of the eight spatial reasoning problems correctly. Overall, greater success was achieved in section one of the test. Seven of the twelve answers given in section one were correct, with four egocentric choices 
selected. In section two, five of the eleven answers given were correct (one student was not given the final problem in this section). Furthermore, no student answered problem 3 or 4 correctly. Section one of the test is presented student by student as the arguments presented tended to be unique to each student. Section two of the test is presented problem by problem as the techniques applied tended to be similar, though the results were quite different.

Section One. Of the three students, Kim was the only one who chose a distractor that was not egocentric. Problem 2 presented a pyramid with a rectangular base cut by an oblique plane. Kim was adamant that the pyramid had a triangular base and chose the sole answer choice that reflected such a shape. The other two students had no difficulty seeing that the base was rectangular and eliminated the triangular choice immediately. Kim was able to choose the correct answers for problems 1 and 3, but chose the egocentric answer for problem 4 . The justification of her answer choice was based on the size of the three-dimensional object in relation to the size of the cross-section. For problem 1 she refuted the egocentric answer, saying "it looks awfully thin for that shape" and referred to that same idea in problem 3. For problem 4, she chose the correct answer because "it looks like the right, um, circumference that, that's shown . . in the picture". Jake chose two correct answers and two egocentric answers. He had no trouble with problem 2 and chose the correct answer quickly without considering any of the distractors. On each of the other three problems, however, he narrowed the choices down to the correct choice and the egocentric choice before making a decision. Similar to Kim, in each case, the deciding factor was how narrow the resulting shape should be. The distinct difference, however, was that Kim referred explicitly to the size in relation to the steepness of the cutting plane. In explaining his choice of the egocentric answer for problem 3, Jake said "the cut is so steep that it looks like 
it would be a, a more narrow cross-section". He made a similar argument for problems 1 and 4 , choosing the correct answer for problem 1 and the egocentric answer for problem 4.

Pete chose three correct answers and one egocentric answer. He initially chose an incorrect answer for problem 2, but when asked to refute the remaining answer choices, changed his answer to the correct choice. Similar to Kim, his justification of his choices for problems 1, 3, and 4 included an argument about the slant of the cutting plane. When selecting an answer for problem 1, he mirrored the cutting plane saying, "I just picked D 'cause it was slanted". For problems 3 and 4, however, he remedied his thinking saying “when you visualize this, you won't notice anything slanting ... you won't notice like an angle or a slant or anything like that". This idea resulted in him choosing the correct answer for both problems.

Section Two. Pete had the most difficulty in the second portion of the test, answering only one problem correctly, while Jake and Kim both answered two problems correctly. Jake tended to present the most focused arguments to justify his choices, though he still resorted to making a best guess on one problem, as the other two students did multiple times.

Though all three students answered problem 1 correctly, Jake was the most successful at justifying his answer choice. Each student began by focusing on a triangular shape in the net. Based on this shape, Jake and Pete were able to eliminate options C and D because, as Jake said, "it's definitely gonna come to a point and neither of these have points". Pete eventually chose A over B, but struggled to make a convincing argument supporting his decision. Kim was also unable to justify her final decision. Early on, she admitted "honestly I'm just kind of guessing cause I, I can't really see the object'. Jake was the only one able to recognize a second defining piece of the net and use it to distinguish between the remaining choices, ultimately choosing the correct answer. 
Pete attempted to employ the same strategy for problem 2 but was unsuccessful. He matched a "V" shape in the net with an incorrect portion of the three-dimensional choices. His incorrect solution was a direct consequence of this misidentification. Jake and Kim began problem 2 by working in the opposite direction - beginning with the three-dimensional objects and eliminating choices based on the absence of relevant net pieces. Each was able to use this strategy to eliminate two answer choices. Kim returned to the original strategy in order to make a final decision, but like Pete, made this decision by incorrectly identifying the "V" shape in the net with a piece of the three-dimensional object. Despite this inaccuracy, she was able to choose the correct answer. Jake was the only student who matched the "V" shape with its correct placement on the three-dimensional objects, though this did not assist in his final decision. In the end, his selection of the correct answer was based on the perceived size of the triangular shape.

None of the students chose the correct answer for problems 3 or 4 . In problem 3 , all three students zeroed in on the same key piece of the net, but all three students misrepresented this piece in the three-dimensional figures. The result was each student choosing the same incorrect answer. Pete was not administered problem 4, while Jake admittedly made a guess at the answer, saying "I don't find any of these in that, honestly". Kim attempted to apply the previous strategies but did not make an appropriate connection between the net and the answer choices.

Physical Volumes. All three students were anxious to cite specific volume methods during the physical volumes portion of the interview. When asked how to find the volume of a donut, each student referenced the "washer method", in which as Kim described, "you take the volume of the whole thing, assuming that this was a solid, but then you would like subtract the, the, the middle". All three students were successful in describing the "outer radius" and "inner radius" of the donut and the washer method process. 
Pete insisted on finding some type of mathematical formula for each object. In the case of the donut, he suggested finding a formula for the surface area and then integrating to get the volume. When trying to find the volume of the banana, he attempted to use a Riemann sum. The butternut squash gave him particular difficulties as he failed to see how to cut it to get a consistent shape to integrate. With some help, he was eventually able to see how it could be broken down into the sum of a cylindrical shape and a spherical shape.

Jake and Kim were able to be more flexible after the initial object and both compared the banana to a cylinder in order to find its volume. The main difference between the two students in this idea was where to measure the "height" of the cylinder - Jake measured along the curve of the inside of the banana while Kim measured along the curve in the center of the banana. Both were aware that this calculation would result in an estimate of the volume, not the exact answer. Similarly, both Jake and Kim quickly recognized the ability to cut the butternut squash into a cylinder and a sphere as a means of estimating its volume.

Solids of Revolution. Jake and Kim had little trouble determining the appropriate integrals for calculating the volumes of the solids of revolution given to them. Both students began by drawing a picture, then using a representative rectangle on their picture to determine which method to use. When asked why he drew a picture, Jake said "It helps me decide what technique to choose for integration". During the process of developing an integral to represent the volume of the solid in problems 2 and 3, Kim actually drew a picture of the representative rectangle rotated about the $y$-axis and referred to the shape as a "shell".

Though she was able to give the correct integral for both problems 2 and 3 (disregarding a bound error), Kim was unable to accurately describe what each piece of the integral represented. For instance, in problem 2 she referred to the function $f(x)$ as the circumference of 
the shell, when it in fact represented the height; she also mistook the radius of the shell, $x$, as representing the height of the shell. She partially corrected this thinking in problem 3 , but still maintained that the radius was in fact the circumference. Kim was able to provide the correct integral for problem 1 and to correctly identify its parts, but admitted that she was just remembering a formula. This lack of understanding was reinforced when she was asked to do problem 2 in a different way. She recognized that the "washer" method applied, but could not remember the formula and could not use her knowledge of the process to reconstruct it.

Jake was able to correctly work all three problems and showed a deep understanding of the process. Unlike Kim, he rarely appeared to be working on memorization. For example, in problem 3, instead of using the "shell" method, he elected to use the "washer" method and split the volume into two parts, similar to the cutting of the butternut squash. In doing so, he had to determine the inner and outer radii for each part, as well as where to make his cut. This determination could not be found using a formula, but took recognizing where the calculations he was going to make required different functions. Specifically, the lower part of the volume required the use of the two functions given, while the upper part of the volume required the line $x=4$.

Pete had some obvious difficulties in determining the volumes, but these difficulties were not related to the process of setting up the integral. Like Jake and Kim, he began by drawing a picture, but struggled to identify the appropriate area to rotate. In problem 2, instead of rotating the area bounded by the given curve and $y=0$, he attempted to rotate the area between the given curve, $x=0$, and $y=3$. The resultant integral represented a volume calculated via the "disc" method, but was of a solid other than the one that was intended. It is important to note, however, that based on Pete's interpretation, the integral was set up correctly. In problem 1, he identified 
the correct portion of the graph to rotate, but elected to use the "washer" method of finding the volume and was unable to determine what his inner radius would be. Despite this issue, he was able to set the integral up correctly, denoting the inner radius by an unknown $g(x)$. Had he made the realization that $g(x)=0$ in this case, he would have presented the correct integral. Pete did not work problem 3 due to time constraints.

\section{Discussion}

Section one of the spatial reasoning test involved examining cross-sections of threedimensional objects. When determining which method to use to compute the volume of a solid of revolution, the student must first determine the shape of a cross-section of the solid. Though not said explicitly, each student made reference to the shape of the cross-section when asked about their choice of variable of integration. For example, while working out problem 2, Jake was asked why he chose to use $y$ as his variable of integration. His response was simple: 'I didn't feel like doing shells". Though he doesn't say it aloud, it is clear that he understood the shape of the three-dimensional object at hand and that slicing it the opposite way would result in "shell"like shapes as opposed to washer-like shapes.

All three students performed well on section one of the spatial reasoning test (taking into consideration that the egocentric answer choice is still essentially correct), and all three students used an appropriate method to calculate each volume based on the picture they developed. This does not necessarily represent a correlation between the two abilities. We can say, however, that a correlation is possible as success was achieved in both areas. Performing this part of the study over a much larger sample size would provide more information about whether these abilities are interdependent. 
Section two of the spatial reasoning test involved forming three-dimensional objects from two-dimensional representations. This is similar to picturing what the solid of revolution will look like based on the two-dimensional section that is rotated. Despite great difficulty in this section of the spatial reasoning test, each student was able to represent a three-dimensional object based on the given two-dimensional section. This discrepancy may lead to the conclusion that a lack of spatial reasoning skills in this area does not affect the student's ability to perform the task at hand. I would argue, however, that either this section of the spatial reasoning test was inadequate for the purpose of the study, or the difficulty level of the solids of revolution was not at a level that truly tested the students' abilities.

Though both tasks involve building three dimensions from two, the process of folding is very different from the process of rotating, so the ability to mentally rotate cannot necessarily be connected to the lack of ability to mentally fold. In addition to this, the spatial reasoning test does not appear to have been formulated based on research, and there is no data to show the validity of the test (it was simply found online). On the other hand, the shapes the students were required to rotate were fairly simple. Producing more complex sections for rotation may have resulted in a better connection between the test and the solids of revolution. Because of these two ideas, this section of data is inconclusive.

Finally, the interviews conducted with Kim and Pete presented a need for further investigation. Though both were successful in setting up the volumes, neither seemed to really understand how they worked. In discussing the physical volumes, Pete said "I never do visualize it this way, she like just gives us graphs and tells us to find the volume which is so easy but I've never looked at it this way". Kim made a similar comment that "I've never thought of the volume of a donut before". Both students struggled to connect the idea of computing volumes of 
solids of revolution to any real-world applications. This lack of connection manifested itself in both of these students relying on the memorization of formulas in computing the volumes of the solids of revolution. This memorization was evidenced in Kim's inability to properly describe what the various pieces of the integral represented and in Pete's inability to see that the inner radius in problem one should have been zero.

\section{Pilot Study 1 Reflection}

This pilot study presented some unexpected challenges. The major challenge was that the students were reluctant to move away from the use of the volume formulas. This seemed to hinder the use of their spatial skills when it came to determining how to find the volume. Because they had knowledge of the volume formulas and were accustomed to being asked to compute volumes that required the use of one of the formulas, the students limited their thinking to determining the answer to one question: "Which formula should be used?". Because of this focus on formulas, it was determined that a different population - one that has not been introduced to the volume formulas - should be used in the future.

A second challenge was the disconnect between the second section of the spatial reasoning test and the results of the calculations. Initially, it was believed that the ability to fold the nets into a three-dimensional shape would parallel the ability to rotate the two-dimensional cross-section to create the three-dimensional solid of revolution. This did not appear to be the case, however. Instead, no correlation between the two tasks was found. Thus, it was determined that a new spatial reasoning test that better reflected the action of rotating the cross-section was needed. 


\section{Pilot Study 2}

A second pilot study was done in the spring of 2015 with students in the first semester of a two-semester Calculus I course.

\section{Methods}

Sixty-seven students ranging in age from 18 to 24 completed a multiple-choice spatial reasoning test consisting of two sections. The first section consisted of fifteen problems taken from the Revised Purdue Spatial Visualization Tests: Visualization of Rotations (Revised PSVT:R). This test was chosen to replace the Spatial Reasoning Test - Hard because it has been shown to be both reliable and valid (Bodner \& Guay, 1997). A variety of questions were chosen from the revised test based on the number of rotations, the axis or axes of rotation involved, and the makeup of the object that is rotated. The second section of the test consisted of ten problems taken from the Santa Barbara Solids Test. The questions for this portion of the test were chosen based on the type of object presented and the axis of the cutting plane.

Following the spatial reasoning test, three students participated in individual interviews to further explore their spatial reasoning skills. Each interview began with a set of warm-up questions to familiarize the student with the interview process and put him or her at ease. Next, the students answered selected questions from the spatial reasoning test and justified their answers. This part of the interview was done without the student referring to their corresponding answers on the written test. If any answer differed from that given on the written test, the student was asked to decide which answer was truly correct and explain why he or she had made a different choice on the written test.

After considering several questions from both sections of the test, the student was asked to determine how to find the volume of a few everyday objects: a donut, a banana, and a 
butternut squash. The students were encouraged to think of splitting the object into more manageable pieces. Finally, the interview concluded with the mathematics portion. This portion contained four problems pertaining to solids of revolution (see Appendix E: Pilot Study 2 Interview Problems). First, the student was asked to sketch the solid of revolution described in each problem. Then, the student was asked to consider how the volume could be found for each solid. In each interview, the researcher began this section with a cursory explanation of how a bounded region is found and how it is rotated about an axis or line. This instruction was necessary as these students had not been exposed to the essential processes to perform the task.

\section{Results}

The mean score for the spatial reasoning test was 14.7 out of 25 questions. This is a good representation of the population as the median score was 14. Test scores ranged from 7 to 25 with 42 students falling within one standard deviation (4.9) of the mean. Overall, students performed slightly better on the Revised PSVT:R portion of the test, scoring a mean of 9.0 (approximately 60\%) to a mean of 5.6 (approximately 56\%) on the SBST portion of the test. Assigning half a point for the egocentric answer on the SBST, however, results in a significant improvement in scores: the overall mean increases to 16.0 with a median of 15.5 and standard deviation of 4.2; the mean score for the SBST portion of the test increases to 6.9 (approximately $69 \%$ ); and the number of students in need of remediation based on the $60 \%$ threshold drops to 31 (approximately 46\%).

Using the $60 \%$ threshold set at Michigan Technological University, approximately half the students ( 35 of 67) were in need of remediation. Breaking it down by test portion, 33 of the students (approximately 49\%) were in need of remediation on PSVT:R skills and 32 of the 
students (approximately 48\%) were in need of remediation on SBST skills. A total of 19 students (approximately 28\%) scored below the $60 \%$ threshold on both portions of the test.

\section{Discussion}

The scores on the spatial reasoning test are significant as the two portions of the test represent the two major components to computing the volume of solids of revolution: rotations and cross-sections. Success on the PSVT:R indicates that the student possesses the ability to perform mental rotations, while success on the SBST indicates that the student possesses the ability to construct cross-sections. Students who struggled on both sections of the spatial reasoning test would likely struggle greatly when asked to compute the volume of a solid of revolution, but even under-development of one of the skills would lead to difficulties. If the student is unable to correctly revolve the bounded region about the given axis, he or she will not have the correct solid for which to compute the volume. If the student is unable to correctly identify the cross-sections of the solid, he or she will not be able to calculate the volume correctly. In order for students to have success in computing volumes of solids of revolution, they must be able to both rotate the bounded region correctly and determine the correct crosssection shape.

Based on the scores of the spatial reasoning test portions, 46 of the 67 students would have trouble computing volumes of solids of revolution due to deficiencies in spatial abilities. The students strong in cross-section abilities but needing development in mental rotations abilities would have a possibility of performing the correct steps for computing volume, but perform those steps on an incorrect figure, therefore resulting in an incorrect answer. Similarly, the students strong in mental rotations abilities but needing development in cross-section abilities would likely create the correct solid of revolution, but use an incorrect formula for volume by 
basing it off of an incorrect cross-section. Students needing development in both abilities would potentially both form an incorrect solid and compute the volume off of incorrect cross sections.

The interviews shed further light on the skills necessary to properly calculate the volume of a solid of revolution. Through the interviews, it became very clear how important the ability to visualize the revolution of a solid is to the process. Though each student interviewed scored at least a 13 out of 15 on the Revised PSVT:R portion of the spatial reasoning test, there were some significant struggles in revolving the bounded region. One of the students interviewed was able to successfully draw each of the figures described. The other two students, however, had a common difficulty in understanding how the solid was formed. Both of these students viewed the solids as a collection of distinct copies of the bounded region placed at various points in the rotation. For instance, one problem asked the student to revolve the region bounded by $f(x)=$ $x^{2}, x=1$, and $y=0$ around the $x$-axis. One student was able to revolve the region correctly, but viewed the "solid" as copies of the bounded region at the original placement and after each 90 degrees of rotation, resulting in a shape similar to a boat propeller.

The difficulties these two students faced are not uncommon. Okumus and Hollebrands (2016) performed a research study with high school students related to revolving twodimensional figures to form solids. In this study, students struggled to translate the rotation of a two-dimensional figure into the resulting three-dimensional figure. At first, using paper and pencil, the students had difficulty translating the directions for rotation into meaningful actions. With the use of physical manipulatives, the students were able to perform the rotations, but were still unable to correctly form the three-dimensional shape. This study further shows that it is important for students not only to understand how to rotate the two-dimensional region, but that they must also understand how the rotation results in a three-dimensional shape. 
When asked to describe how to compute the volume of each solid none of the students used a cross-section approach. This is likely due to the fact that the students had no experience with integration or the idea of cutting a figure into cross-sections. Each student compared the figures to objects with known formulas, such as a sphere, pyramid, cylinder, etc. The students were aware of the fact that using one of these formulas would not result in an exact answer, as the figures were not exact. The strategy, however, was to either get an estimate of the volume, or use the known formula and subtract some piece of the volume away. For example, one student noted a pyramid-like shape but with curved sides, so it was determined that the pyramid formula would be used, then somehow the difference between the straight sides of the pyramid and the curved sides of the actual solid would be subtracted out to give the actual volume.

\section{Pilot Study 2 Reflection}

This study had major impacts on the direction of the next phase of research. By using students in a lower-level calculus course, the problem in the previous study of students relying on the volume formulas was eliminated. This showed that the population was a good choice for the research because bias from previous knowledge was eliminated, as most of the students have not had calculus previously, and the students were forced to rely on spatial skills to complete the problems.

This population also presented some new issues. Students had to be instructed on how to rotate the two-dimensional region to get a three-dimensional solid. Because time was limited and the concept was so new, some students struggled to understand how the rotation worked, therefore resulting in, at times, an object that could not be considered a solid. This essentially eliminated the relevance of the second portion of the interview because there is no way to determine a cross-section or find a volume when the object is not a solid. Also, as noted above, 
none of the students took a cross-section approach to computing volume, likely because they had never seen volumes computed in such a manner. This rendered the students' scores on the SBST completely irrelevant. This knowledge guided the development of the research design for the next phase of research. 


\section{Research Methods}

The dissertation research was a mixed-methods study using the combination of a quantitative spatial reasoning test, semi-structured interviews, and student activities. The design of the study was pre-test - post-test matched subject, using the quantitative spatial reasoning test - the Santa Barbara Solids Test (SBST) - and other demographic information to match students of similar spatial reasoning abilities and backgrounds for the qualitative part of the study.

\section{Population}

Research was done with students enrolled in a two-semester Calculus I course at West Virginia University (WVU). Research and analysis were performed under the WVU Institutional Review Board (IRB) protocols numbered 1412515061, 1705602250, and 2002883396. Student identities are protected by using pseudonyms in place of names. In total, 306 students took both the pre-test and post-test (see Figure 5). Students who did not take both tests were not included in the dissertation research. The students ranged in age from 17 to 29 , with over half the students aged 18, and fewer than 20 students older than 20. The split between males and females was fairly even, with the group containing 58 more males than females.

Students participating in the study are referred to as part of six groups: the Total Population, the General Population, the Below-Average Population, the Interview Population, the Haptic Group, and the Non-Haptic Group (see Figure 6). The Total Population was comprised of all students in a Calculus I course that had been designed to be taught in two semesters. Following the SBST pre-test, the Total Population was divided into the General Population and the Interview Population. 


\section{Figure 5}

Demographics for the Total Population

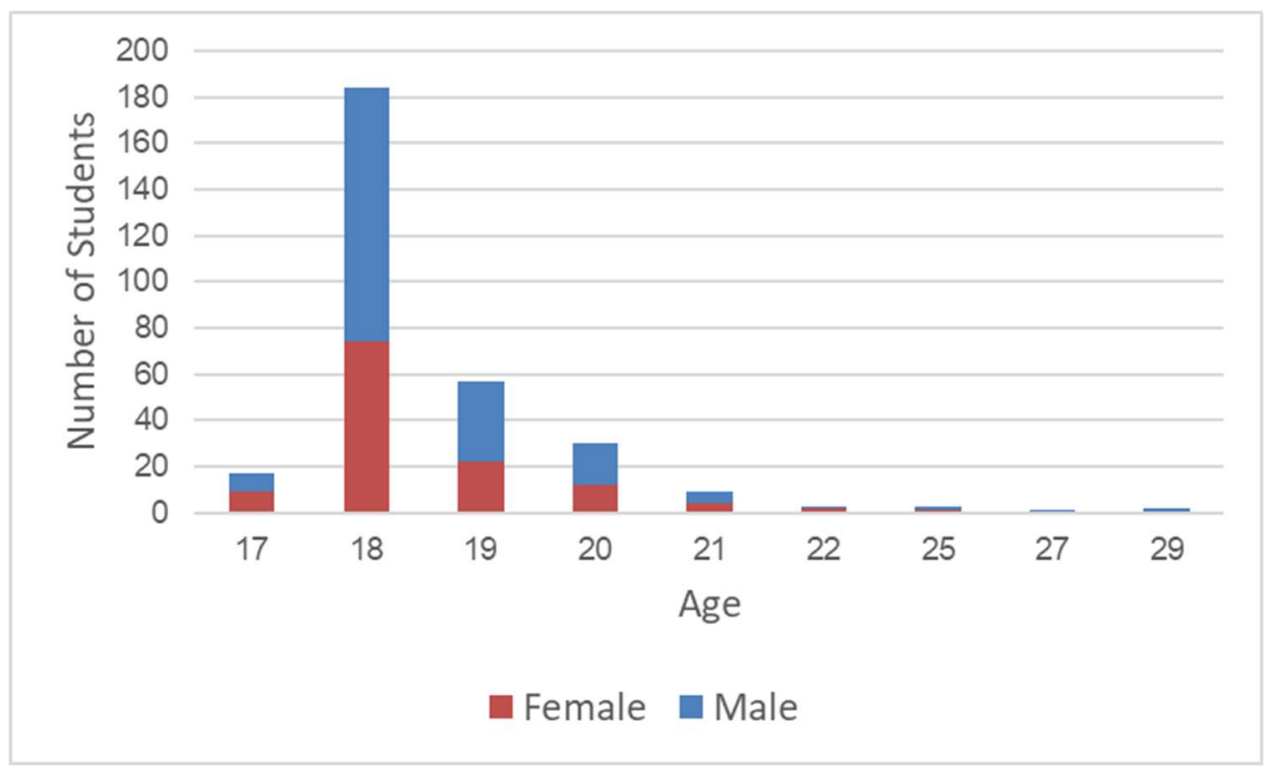

Figure 6

The Six Student Groups

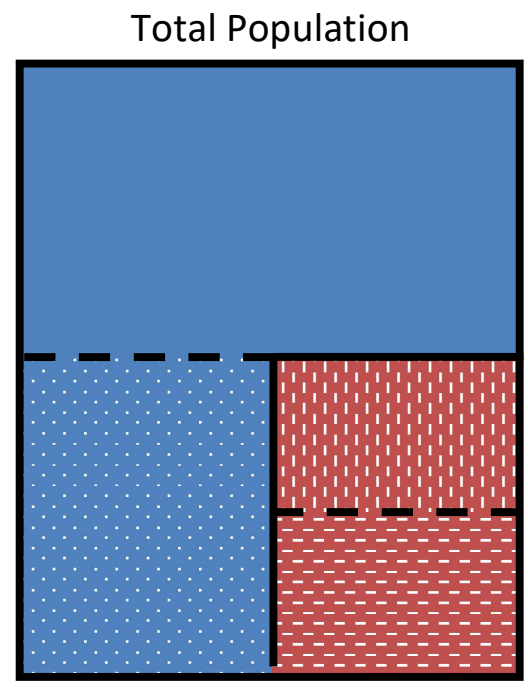

General Population

$\therefore$ Below-Average Population

$\square$ Interview Population

Ni, Non-Haptic Group

=- Haptic Group 
To determine the Interview Population that would be divided into the Haptic and NonHaptic Groups, information learned from the pilot study and the literature were considered. During the pilot studies, it was discovered that once the students had been introduced to common methods for calculating the volumes of solids of revolution, such as the Disc Method, the Washer Method and the Shell Method, the focus tended to be on using one of the formulas and algebraic manipulation, rather than thinking critically about the solids and their cross sections when cut by a plane. This hindered the ability to analyze the student's spatial reasoning abilities during the interview. The same difficulty was presented during the pre- and post-interviews, so it was imperative that the students being interviewed had not been introduced to these common calculus methods.

In the two-semester Calculus I course at WVU, students do not cover volumes of solids of revolution, but it was also possible that some students may have taken a calculus course previously, and consequently may have encountered the common methods of finding these volumes in the past. Part of the demographic information collected from students was previous mathematics courses, with the goal of choosing only students who had not taken calculus at the high school or college level in the past. This was not possible, so six of the ten students who agreed to participate had seen some form of calculus. None of the students, however, appeared to have had experience in calculating volumes of solids of revolution.

Based on findings from the literature review, the Interview Population was initially comprised of five pairs of students taken from the group of students who scored below the average on the pre-test. It has been shown that students with lower spatial abilities benefit more from targeted interventions than students with higher spatial abilities (Lee \& Wong, 2014). The goal of limiting the interviews and observations to students with lower spatial abilities was to 
have the opportunity to observe a larger increase or a more prominent effect from the intervention.

To form the Interview Population, five students were chosen at random and pairs were formed by matching five additional students based on their sex, age, previous math courses, and SBST pre-test score. Some of the students initially chosen elected not to participate in the study, so alternate matches were chosen until five pairs who agreed to participate were identified. These ten students became the Interview Population. From each pair, one student was randomly placed in the Haptic Group and the other was placed in the Non-Haptic Group.

Prior to beginning the intervention phase, one student in the Non-Haptic Group dropped out of the study, leaving four matched pairs and one additional student in the Haptic Group. This unmatched student completed the entire study, and as such, remained a part of the Interview Population and Haptic Group for all analysis except for the matched-pair analysis. The student who left the study did so prior to completing any of the intervention work, so his test scores were considered a part of the General Population during analysis.

The students who scored below-average on the pre-test but were not a part of the Interview Population comprised the Below-Average Population. This subset of the General Population was identified for analytic purposes as a way to compare the Interview Population to the subset of the Total Population the participants were chosen from.

\section{Research Design}

The study was done in three phases - the pre-test phase, the intervention phase, and the post-test phase - over the course of a semester. The pre-test phase consisted of a SBST pre-test followed by a semi-structured pre-interview. First, the SBST was given to the Total Population. To avoid poor data due to lack of effort, measures were taken to help ensure the students took the 
tests seriously, including proctoring the testing sessions and giving participating students bonus points. Based on these SBST pre-test scores, five pairs of students were chosen to make up the Interview Population (split into the Haptic and Non-Haptic Groups as described above).

After the Interview Population was determined, a semi-structured pre-interview was conducted with each student in the group (see Appendix F: Interview Protocol). The interview consisted of four parts. First, the student was asked some warm-up questions about his or her background in order to ease him or her into the interview setting. Second, the student was given three questions from the SBST and asked to explain how he or she chose each answer. Next, the interviewer showed the student a few three-dimensional objects. The student was first asked to draw the cross-section of the object, similar to the SBST, but without the multiple-choice format. Then, the student was asked to think critically to determine which cutting plane, parallel to the $x$ axis or parallel to the $y$-axis, should be used in order to make it easiest to calculate the area for all cross-sections of the object. Finally, the student was asked to draw a three-dimensional object given some information about its cross-sections. The student was given two pieces of information about the object's cross-sections: all cross-sections determined by a vertical cutting plane (through one plane) have a specific shape, and most, but not all, cross-sections determined by a horizontal cutting plane have a specific shape. Through each of these questions, the student was asked to talk through his or her thought process and explain the answer. These interviews completed the pre-test phase.

During the intervention phase, the members of the Haptic and Non-Haptic Groups worked through four activities. During these activities, both groups completed practice problems similar to those presented in the pre-interview. Students in the Haptic Group worked through these problems while viewing and "feeling" the objects on the computer with the haptic device. 
Students in the Non-Haptic Group worked through the problems while viewing the objects on the computer screen, but did not have access to haptic feedback. Interaction between the students and the researcher was limited during these activities, with the researcher or research assistant available to answer questions to clarify the assignment or assist with the technology. The researcher and research assistant did not provide any instruction during this time.

Finally, during the post-test phase, the pre-test phase was repeated in reverse order. First, the students in the Interview Population completed the semi-structured post-interview. This interview followed a similar protocol to the pre-interview; instead of asking the student background questions, he or she was asked for feedback about the intervention design, and the cross sections used to define the three-dimensional shape in the last problem were modified. The final problem was also inadvertently modified so that all horizontal cross-sections were one shape, while the vertical cross-sections required some variation. To conclude the study, all students in the Total Population took the SBST again. The same setting used during the pre-test was used during the post-test to encourage students to take the test seriously and bonus was also provided for the post-test.

\section{Intervention Design}

The intervention consisted of four applications and worksheets developed specifically for this research study and was completed over a four-week period in small groups. Students met once per week in a classroom setting, where all computers and haptic devices (if necessary) were set up and ready to use. The Non-Haptic Group met all together, while the Haptic Group met over two time periods due to there being fewer haptic controllers than students in the group. The two groups met on separate days in an attempt to prevent cross-contamination between the groups. All intervention meetings were overseen by a research assistant who was available to 
answer technical questions related to the applications, but was instructed not to assist the students in the completion of the activities.

\section{Applications}

The applications were developed using a similar framework employed for other educational haptics applications (Darrah et al., 2014). Each application had two versions - a nonhaptic version used by the Non-Haptic Group and a haptic version used by the Haptic Group. Aside from the additional haptic functionality described below, the two versions of each application were identical. All the applications were loaded onto laptop computers provided by the university. The haptic version of the applications also required the use of the Novint Falcon as a peripheral.

The Novint Falcon (see Figure 7) is a low-cost haptic controller designed for computer gaming. The device has three degrees of freedom. To control the cursor, the user grasps a spherical knob that contains four buttons that provide feedback to the computer. When grasping the knob, the user can feel the haptic feedback from the device, including, but not limited to, shape, weight, magnetism, and texture. The user is able to "feel" objects through force feedback provided by the device. Although some devices are still available through a third-party vendor, development and support for the controller has ceased. 


\section{Figure 7}

The Novint Falcon Haptic Controller

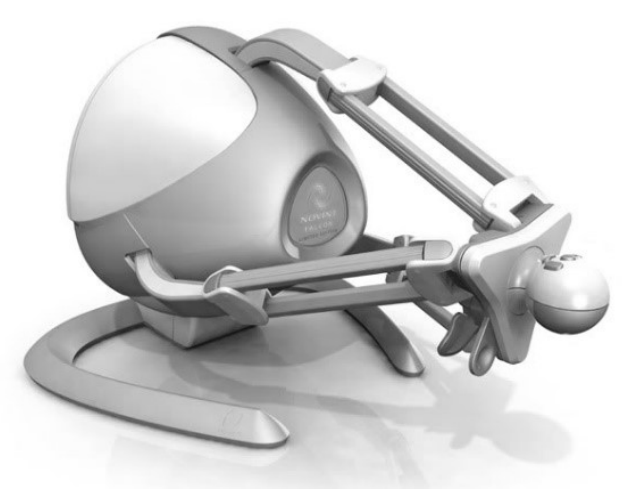

All of the applications followed the same format: three scenes, each with one threedimensional object for the student to analyze. Each student was provided a reference sheet with the relevant commands for the applications (see Appendix G: Application Directions - With Haptics and Appendix H: Application Directions - Without Haptics). The student moved from scene to scene by pressing the "TAB" key to move to the next scene, or the "BACKSPACE" key to move to the previous scene. The camera position could be rotated around the scene by using the arrow keys. This allowed the student to view the object from different angles in order to give him or her as clear a picture as possible of what the object looked like. To return the camera to its original position, the student could press the spacebar.

Pressing the "X" or "Y" key displayed two cutting planes on the object, parallel to the selected axis (see Figure 8 and Figure 9). Each cutting plane was numbered to direct the student in the completion of the worksheets that accompanied the activities (see Worksheets below). Similar to the camera, the student could press the spacebar to remove the cutting planes and return the figure back to its original form. To exit the application, the student could press the "ESC" key. 


\section{Figure 8}

An Object With Horizontal Cutting Planes (After Pressing the "X” Key)

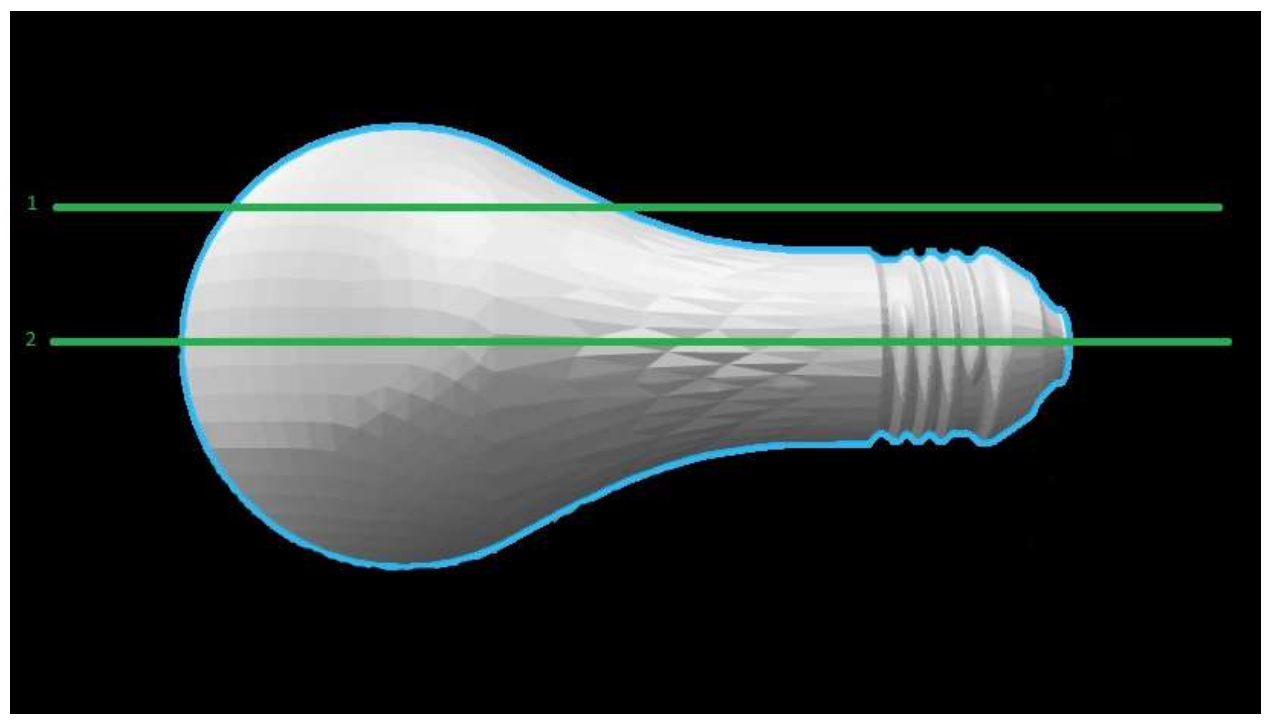

Figure 9

An Object With Vertical Cutting Planes (After Pressing the "Y” Key)

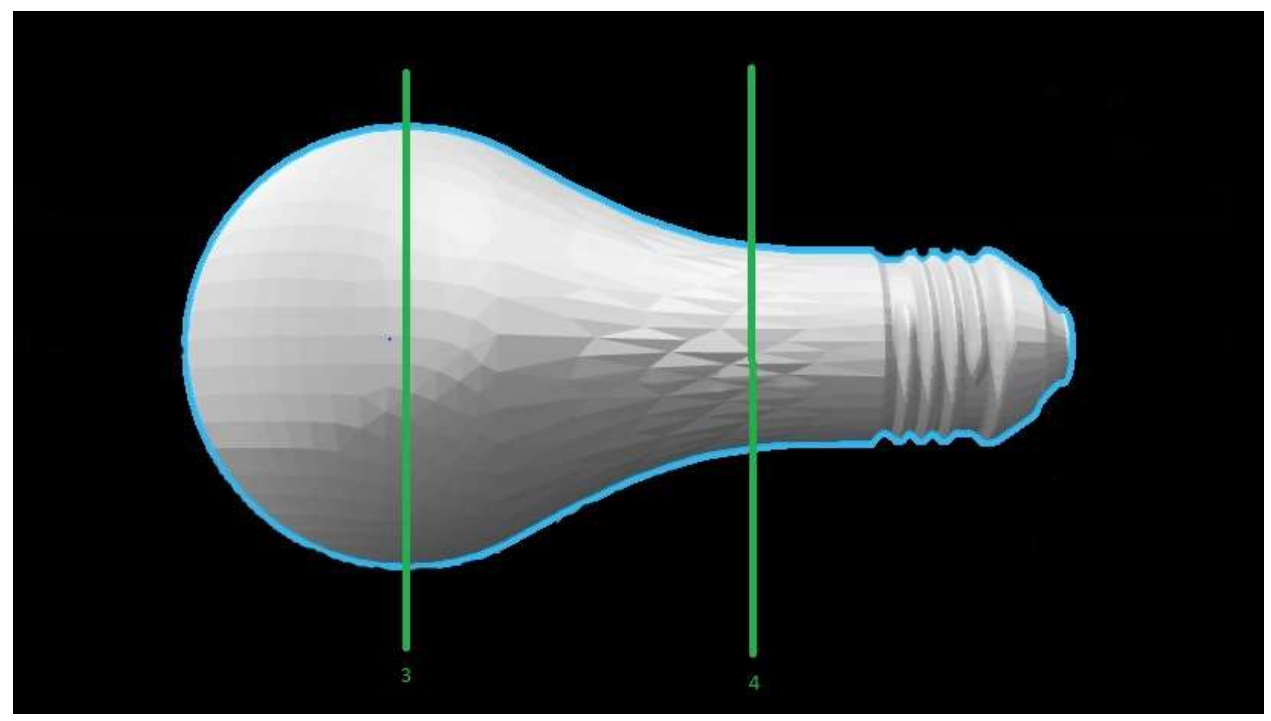


The haptic version of each application added additional functionality to allow the student to feel the objects. The student was able to explore each scene freely and "touch" each object using the haptic device. In addition to this, when cutting planes were present (by pressing the "X" or "Y" key, as described above), the student could press the number key corresponding to a specific cross-section and have the movement of the haptic device restricted to that cutting plane. This allowed the student to trace the perimeter of the cross-section created by slicing the object with the chosen cutting plane.

In addition to the four intervention applications, the Haptic Group also completed a preliminary application. This application served as an introduction to the haptic device and environment and was completed in the first week of the intervention, prior to the first intervention activity. This application was given to the Haptic Group to help the students grow accustomed to the "feel" of different objects in the haptic environment and develop a familiarity with navigation. Each scene contained one simple 3-D object, such as a sphere, a cylinder, or a cube. Similar to the intervention activities, the student was able to explore the scene freely and "touch" the object using the haptic device.

In this initial application, for each scene except the last, the student was able to see the object being touched. In the final scene, the student was not able to see the object being touched. The goal of this last scene was to test the student's attentiveness to how the objects feel in the haptic environment, so the student was encouraged to make a conjecture about what object he or she was touching. As a check, the student was able to view the object by pressing the "V" key. As added practice in this last exercise, the student was able to move to a new object, chosen randomly from a fixed set of objects, by pressing the "N" key. This again produced a scene with no visual, but something to feel. 


\section{Worksheets}

Each intervention application was accompanied by a worksheet for the student to complete (see Appendix I: Sample Activity for an example). The worksheets were designed to be similar to the interview questions pertaining to the real objects and were identical across the groups. The same five questions were presented for each scene. First, the student was asked to make a three-dimensional sketch of the object. Next, the student was instructed to press the " $\mathrm{X}$ " key to see horizontal cutting planes, and to draw a picture of the cross-section created by each cutting plane. The same question was asked for the vertical cutting planes, viewed by pressing the "Y" key.

Next, the student was asked to imagine multiple cutting planes on the object in each direction (parallel to the $x$-axis and parallel to the $y$-axis), and to make a decision on which cutting plane direction should be used to make it easiest to calculate the areas of all the resulting cross-sections. Because of the various reasons for choosing one method or the other, the student was asked to explain why he or she chose that particular cutting plane direction. Finally, the student was asked to give a list of measurements needed in order to calculate the areas of the cross-sections that would result from his or her chosen cutting plane direction, and to explain the answer.

The final activity included four additional questions to garner feedback on the intervention. Students were asked what the most beneficial part of the application was and why, as well as for their overall impression of the applications including any specific likes, dislikes, and suggestions for improvement. Students were also asked to reflect on how much they interacted with the objects (even the Non-Haptic Group could interact with the objects by moving the camera) and what prevented them from interacting with the objects if they did not 
interact much. Finally, the students were asked if they had any difficulties while using the application and how they could have been alleviated.

\section{Data Analysis}

\section{Pre-Test and Post-Test}

The SBST was scored out of 60 points - two points for each correct answer, one point for each egocentric answer, and zero points for each incorrect answer. For the pre-test, the mean over the Total Population was found in order to select students for the Interview Population. The median and standard deviation were also found in order to provide further analysis of the pre-test data, as these scores provided the baseline for the knowledge students entering calculus possess. Following the post-test, the scores of any student who did not complete both the pre-test and post-test were removed from the data sets. This allowed three sets of scores to be calculated for each student: the pre-test scores, the post-test scores, and the change scores.

The change scores were found by taking the difference between the post-test and pre-test scores, with a positive change score representing an increase from the pre-test to the post-test and a negative change score representing a decrease from the pre-test to the post-test. These scores represent the improvement in spatial knowledge between interviews. Extreme change scores could indicate a lack of effort on one test or the other, resulting in a skewed score. Enough of these scores could result in an inaccurate analysis due to the average score being influenced by extreme outliers. To determine whether averages were skewed by extreme scores, a test analysis was performed after eliminating the extreme $5 \%$ and $10 \%$ of the scores and these numbers were compared to the total population. The average values did not change significantly, indicating that the outliers had little effect on the calculations. Also, as indicated earlier, measures were taken to 
encourage the students to take both the pre-test and post-test seriously. For these reasons, no reduction to the total population was made during analysis.

Multiple paired t-tests were performed on the pre-test and post-test scores. Paired twosample t-tests were run on each population to compare the pre-test data (number of correct answers, number of egocentric answers, and total score) to the post-test data. These tests showed the change in scores amongst each population. Paired two-sample t-tests were also run on the matched pairs to compare the pre-test, post-test, and change scores. The test on the pre-test values was used to verify that the groups were not significantly different, while the tests on the post-test and change scores showed the difference in performance between the Haptic and NonHaptic groups.

Two-sample t-tests with unequal variance were run on the General Population and Interview Population to verify that the pre-test values for the two populations were significantly different, and to determine whether the post-test values were significantly different. Similarly, two-sample t-tests with unequal variance were run on the Below-Average Population and the Interview Population to verify that the pre-test values for the two populations were not significantly different, and to determine whether the post-test values were significantly different. Finally, two-sample t-tests were run on the General Population and the Interview Population and the Below-Average Population and the Interview Population to determine whether the change scores between the populations were significantly different.

\section{Pre-Interview and Post-Interview}

Interview analysis was done only on the eight students who were part of the matched pairs (one student in the Non-Haptic Group dropped out prior to the intervention phase). The interviews were analyzed using both inductive and deductive coding. Deductive coding is 
performed by creating the codes prior to examining the data (Braun \& Clarke, 2006), then looking for elements of the data that specifically fit those codes. Student explanations were examined for spatial language using the Spatial Framework developed by Engelke et al. (2016) and coded based on the sixteen categories of the framework. Inductive coding is a method of coding in which the codes emerge from the data, instead of being pre-determined (Braun \& Clarke, 2006). This was done to note any other significant characteristics in the interviews, such as the classification of toys and sports into high and low spatial needs categories. Coding of the pre-interviews shed light on the baseline knowledge of the interview population. Coding of the post-interviews was used to analyze the improvement of the interview population and to compare the Haptic and Non-Haptic groups to help determine the effect of the haptic intervention.

The SBST questions and student drawings from the interviews were scored out of 24 points (see Figure 10). Each SBST question received 2 points for the correct answer, 1 point for the egocentric answer, and 0 points otherwise (as was done in the pre-test). Each drawing for the sponge and duct tape cross-sections received 2 points for the correct answer, 1 point if only one misconception existed, and 0 points otherwise. The lightbulb cross-section drawings were not scored, as this exercise was omitted from some interviews due to time constraints and the answers given for this exercise did not provide much additional insight. 


\section{Figure 10}

Pre-Interview and Post-Interview SBST and Student Drawings Rubric

\begin{tabular}{llc} 
Point value & Rubric description & Maximum score \\
\hline SBST Questions (3 questions) & $\mathbf{6}$ \\
2 & Correct Answer & \\
1 & Egocentric Answer & $\mathbf{1 2}$ \\
0 & Incorrect Answer & \\
Physical & Objects (2 objects x 3 questions per object) \\
2 & Correct Drawing & \\
1 & One Misconception & $\mathbf{6}$ \\
0 & More than one misconception & 2 \\
Complex Figure & \\
Horizontal Cross-sections & \\
2 & All requirements met \\
1 & Some incorrect cross-sections & 3 \\
0 & No requirements met & \\
Vertical Cross-sections & All requirements met \\
3 & One requirement not met & \\
2 & Two requirements not met & \\
1 & No requirements met
\end{tabular}

The final interview activity was scored out of 6 points. Students received up to 2 points for drawing a figure that met the horizontal cutting plane conditions. Students received up to 3 points for drawing a figure that met the vertical cutting plane conditions. Finally, students received 1 point for developing a cohesive drawing, that incorporated both horizontal and vertical cutting plane ideas (even if all requirements were not met). The breakdown of points for the interview artifacts is as follows: up to 6 points for SBST answers, up to 6 points for the sponge cross-sections, up to 6 points for the duct tape cross-sections, and up to 6 points for the complex figure. 


\section{Intervention Activities}

The intervention activity worksheets were not analyzed due to the exploratory nature of the intervention. The research questions focus on changes in behavior from the pre-interview to the post-interview and from the pre-test to the post-test, so analysis of the intervention activities would not provide any relevant information to assist in answering the research questions. In the future, it may be interesting to analyze the intervention activity worksheets and examine the differences between the haptic and non-haptic groups, as well as differences from the first intervention exercise to the last. 


\section{Data Analysis}

\section{Pre-test and Post-test Scores}

In total, 306 students completed both the SBST pre-test and post-test. Scores were analyzed across the six groups mentioned previously in the Methods section. Some basic analyses on each set of scores were done for each group by examining the maximum, minimum, average, and median values. For the Total Population, this basic analysis of the pre-test scores served as a baseline for students entering calculus. On the pre-test, the Total population had an average overall score of 45.1 , breaking down into an average of 20 correct answers chosen and 5.2 egocentric answers chosen (the discrepancy in the calculation of the overall score from the individual answers is due to rounding).

More in-depth analyses were done on and between the five sub-groups. Paired twosample $t$-tests were performed on the pre-test and post-test data in each group to determine whether changes from the pre-test to the post-test were significant. For these tests, one-tailed $p$ values were used, as the expectation was that the number of correct answers and the total score would increase, while the number of egocentric answers would decrease. Paired two-sample $t$ tests were also performed between the matched pairs, and two-sample $t$-tests of unequal variance were performed between some of the groups. Each of these tests used two-tailed $p$-values, as the goal was to determine whether there was any difference between the two groups.

The average pre-test and post-test scores for all groups can be seen in Table 1, along with the relevant $p$-values. Note that as the correct answers increase, it is natural that the egocentric answers decrease. From the pre-test to the post-test, each group increased its average number of correct answers and its average total score, while decreasing the average number of egocentric answers. For the General Population, each of these changes was too small to be considered 
significant. For the remaining populations, all changes were considered significant, with the exception of the decrease in the number of egocentric answers chosen by the Below-Average Population. The most significant changes were found in the Interview Population. For this group, the $p$-value for each category (correct answers, egocentric answers, total score) was less than .001 , indicating the changes were highly significant.

\section{Table 1}

Paired Two-Sample t-Tests for Pre-Test and Post-Test Scores, with One-Tailed p-Values

\begin{tabular}{|c|c|c|c|c|c|c|c|}
\hline \multirow[t]{2}{*}{ Group } & \multirow[t]{2}{*}{$n$} & \multicolumn{2}{|c|}{ Pre-test } & \multicolumn{2}{|c|}{ Post-test } & \multirow[t]{2}{*}{$t$} & \multirow[t]{2}{*}{$p^{\mathbf{a}}$} \\
\hline & & $\mathbf{M}$ & SD & $\mathbf{M}$ & SD & & \\
\hline \multicolumn{8}{|c|}{ Number of Correct Answers } \\
\hline GP & 297 & 20.1 & 6.2 & 20.5 & 6.8 & 1.42 & .078 \\
\hline BAP & 130 & 14.6 & 4.5 & 15.6 & 6.5 & 2.29 & .012 \\
\hline IP & 9 & 14.2 & 3.5 & 20.9 & 3.7 & 5.21 & $<.001$ \\
\hline NHG & 4 & 13.8 & 3.5 & 20.3 & 5.3 & 3.81 & .016 \\
\hline HG & 5 & 14.6 & 3.8 & 21.4 & 2.5 & 3.34 & .014 \\
\hline \multicolumn{8}{|c|}{ Number of Egocentric Answers } \\
\hline GP & 297 & 5.0 & 4.9 & 4.7 & 5.1 & -1.45 & .074 \\
\hline BAP & 130 & 8.1 & 5.3 & 7.6 & 5.9 & -0.09 & .089 \\
\hline IP & 9 & 10.1 & 3.1 & 4.6 & 4.2 & -4.57 & $<.001$ \\
\hline NHG & 4 & 11.0 & 2.2 & 5.5 & 4.8 & -3.54 & .019 \\
\hline $\mathrm{HG}$ & 5 & 9.4 & 3.8 & 3.8 & 4.0 & -2.85 & .023 \\
\hline \multicolumn{8}{|c|}{ Total Score } \\
\hline GP & 297 & 45.3 & 8.5 & 45.7 & 9.5 & 1.06 & .144 \\
\hline BAP & 130 & 37.3 & 5.3 & 38.7 & 8.7 & 2.32 & .011 \\
\hline IP & 9 & 38.6 & 4.8 & 46.3 & 3.6 & 5.10 & $<.001$ \\
\hline NHG & 4 & 38.5 & 5.3 & 46.0 & 5.7 & 3.33 & .022 \\
\hline HG & 5 & 38.6 & 5.0 & 46.6 & 1.3 & 3.47 & .013 \\
\hline
\end{tabular}

Note. $\mathrm{GP}=$ General Population. BAP $=$ Below-Average Population. IP = Interview Population. $\mathrm{NHG}=$ Non-Haptic Group. HG = Haptic Group.

${ }^{\text {a }}$ one-tailed $p$-values were used, as the expectation was that the number of correct answers and the total score would increase from the pre-test to the post-test, while the number of egocentric answers would decrease 
In order to determine whether the haptics played a significant role in improving spatial skills, paired two-sample $t$-tests were run on the matched pairs between the Non-Haptic and Haptic Groups for the pre-test scores, post-test scores and change scores. The average values for each group and the two-tailed $p$-value as determined by each $t$-test can be seen in Table 2 . By design, the Haptic Group and Non-Haptic Group had nearly identical scores on the pre-test. This was desired, as the groups were formed using matched pairs. The two-tailed $p$-values indicate that the small differences between the groups were not significant, indicating that the students were at the same level of performance during the pre-test, so any differences in the post-test can be attributed to the presence or absence of haptics in the intervention.

\section{Table 2}

Paired Two-Sample t-Tests for Matched Pairs (n=4), with Two-Tailed p-Values

\begin{tabular}{|c|c|c|c|c|c|c|}
\hline \multirow[t]{2}{*}{ Score } & \multicolumn{2}{|c|}{ Non-Haptic Group } & \multicolumn{2}{|c|}{ Haptic Group } & \multirow[t]{2}{*}{$t$} & \multirow[t]{2}{*}{$p^{\mathbf{a}}$} \\
\hline & $\mathbf{M}$ & SD & $\mathbf{M}$ & SD & & \\
\hline \multicolumn{7}{|c|}{ Pre-Test } \\
\hline Number Correct & 13.8 & 3.5 & 13.8 & 3.8 & 0.00 & 1.000 \\
\hline Number Egocentric & 11.0 & 2.2 & 10.8 & 2.8 & -0.40 & .718 \\
\hline Total Score & 38.5 & 5.3 & 38.3 & 5.7 & -1.00 & .391 \\
\hline \multicolumn{7}{|c|}{ Post-Test } \\
\hline Number Correct & 20.3 & 5.3 & 21.3 & 2.9 & 0.37 & .739 \\
\hline Number Egocentric & 5.5 & 4.8 & 4.3 & 4.5 & -0.39 & .720 \\
\hline Total Score & 46.0 & 5.7 & 46.8 & 1.5 & 0.30 & .781 \\
\hline \multicolumn{7}{|c|}{ Change Score } \\
\hline Number Correct & 6.5 & 3.4 & 7.5 & 4.9 & 0.33 & .763 \\
\hline Number Egocentric & -5.5 & 3.1 & -6.5 & 4.5 & -0.29 & .792 \\
\hline Total Score & 7.5 & 4.5 & 8.5 & 5.8 & 0.37 & .736 \\
\hline
\end{tabular}

Note. One student from the Haptic Group was not included in this analysis due to the student's matched pair dropping from the study prior to the intervention phase.

${ }^{\text {a }}$ two-tailed $p$-values were used, as the goal was to determine whether there was any difference between the two groups 
Two-sample $t$-tests with unequal variance were performed between the General Population and the Interview Population (drawn from the lower half of the general population) to determine whether the differences in the pre-test and post-test scores were significant. The pretest and post-test scores accompanied by the two-tailed $p$-values are displayed in Table 3. Differences on the pre-test are considered highly significant, with $p$-values of .002 or less, while differences on the post-test are not considered significant. This means that while the Interview Population began the study distinct from the General Population, after completing the intervention, they were brought to the level of their peers.

Two-sample $t$-tests with unequal variance were also performed between the BelowAverage Population and the Interview Population to determine whether the differences in the pre-test and post-test scores were significant. The average values and the two-tailed $p$-values from the $t$-tests are shown in Table 4. These results are almost opposite those of the General Population vs. the Interview Population.

On the pre-test, the Below-Average Population and Interview Population did not perform significantly different in any of the scores. On the post-test, however, the groups performed significantly different on the number of correct answers and the total score, as indicated by twotailed $p$-values of .002 or less. This means that while the Interview Population began the study at a similar level to the Below-Average Population, by the conclusion of the study, the group had set themselves apart and scored significantly higher. An interesting note is that the groups were not significantly different on the pre-test or post-test when it came to the number of egocentric answers chosen. 


\section{Table 3}

Two-Sample t-Tests with Unequal Variance for General Population and Interview Population Pre-Test and Post-Test Scores, with Two-Tailed p-Values

\begin{tabular}{|c|c|c|c|c|c|c|}
\hline \multirow[t]{2}{*}{ Score } & \multicolumn{2}{|c|}{$\begin{array}{c}\text { General Population } \\
(n=297)\end{array}$} & \multicolumn{2}{|c|}{$\begin{array}{l}\text { Interview Population } \\
\qquad(n=9)\end{array}$} & \multirow[t]{2}{*}{$t$} & \multirow[t]{2}{*}{$p^{a}$} \\
\hline & $\mathbf{M}$ & SD & $\mathbf{M}$ & SD & & \\
\hline \multicolumn{7}{|c|}{ Pre-Test } \\
\hline Number Correct & 20.1 & 6.2 & 14.2 & 3.5 & 4.90 & $<.001$ \\
\hline Number Egocentric & 5.0 & 4.9 & 10.1 & 3.1 & -4.68 & .001 \\
\hline Total Score & 45.3 & 8.5 & 38.6 & 4.8 & 4.03 & .002 \\
\hline \multicolumn{7}{|c|}{ Post-Test } \\
\hline Number Correct & 20.5 & 6.8 & 20.9 & 3.7 & 0.31 & .763 \\
\hline Number Egocentric & 4.7 & 5.1 & 4.6 & 4.2 & -0.11 & .916 \\
\hline Total Score & 45.7 & 9.5 & 46.3 & 3.6 & 0.49 & .633 \\
\hline
\end{tabular}

${ }^{a}$ two-tailed $p$-values were used, as the goal was to determine whether there was any difference between the groups

\section{Table 4}

Two-Sample t-Tests with Unequal Variance for Below-Average Population and Interview Population Pre-Test and Post-Test Scores, with Two-Tailed p-Values

\begin{tabular}{|c|c|c|c|c|c|c|}
\hline \multirow[t]{2}{*}{ Score } & \multicolumn{2}{|c|}{$\begin{array}{c}\text { Below-Average } \\
\text { Population } \\
(n=130) \\
\end{array}$} & \multicolumn{2}{|c|}{$\begin{array}{l}\text { Interview Population } \\
\qquad(n=9)\end{array}$} & \multirow[t]{2}{*}{$t$} & \multirow[t]{2}{*}{$p^{\mathbf{a}}$} \\
\hline & $\mathbf{M}$ & SD & $\bar{M}$ & SD & & \\
\hline \multicolumn{7}{|c|}{ Pre-Test } \\
\hline Number Correct & 14.6 & 19.9 & 14.2 & 3.5 & 0.29 & .781 \\
\hline Number Egocentric & 8.1 & 27.6 & 10.1 & 3.1 & -1.72 & .114 \\
\hline Total Score & 37.3 & 28.3 & 38.6 & 4.8 & 0.76 & .465 \\
\hline \multicolumn{7}{|c|}{ Post-Test } \\
\hline Number Correct & 15.6 & 41.7 & 20.9 & 3.7 & 3.89 & .002 \\
\hline Number Egocentric & 7.6 & 34.4 & 4.6 & 4.2 & -2.02 & .071 \\
\hline Total Score & 38.7 & 75.0 & 46.3 & 3.6 & 5.31 & $<.001$ \\
\hline
\end{tabular}

a two-tailed $p$-values were used, as the goal was to determine whether there was any difference between the two groups 
To determine whether the Interview Population improved significantly more than the Below-Average population, two-sample $t$-tests with unequal variance were also performed on the change scores for the Below-Average Population and the Interview Population. The change scores and their associated two-tailed $p$-values are shown in Table 5. Each of the change scores for the Interview Population is considered significantly better than each of the change scores for the Below-Average Population. This indicates that participating in the intervention caused a more significant change in the test scores for the students who scored below average on the pretest (recall that the Interview Population was pulled from this group of students).

\section{Table 5}

Two-Sample t-Tests with Unequal Variance for Below-Average Population and Interview Population Change Scores, with Two-Tailed p-Values

\begin{tabular}{lcccccc}
\hline \multicolumn{1}{c}{ Score } & $\begin{array}{c}\text { Below-Average } \\
\text { Population } \\
(\boldsymbol{n}=\mathbf{1 3 0})\end{array}$ & $\begin{array}{c}\text { Interview Population } \\
(\boldsymbol{n}=\mathbf{9})\end{array}$ & $\boldsymbol{t}$ & \multirow{2}{*}{$\boldsymbol{p}^{\mathbf{a}}$} \\
\cline { 2 - 6 } & $\mathbf{M}$ & $\mathbf{S D}$ & $\mathbf{M}$ & $\mathbf{S D}$ & & \\
\hline Number Correct & 1.0 & 25.6 & 6.7 & 3.8 & 4.17 & .002 \\
Number Egocentric & -0.6 & 24.2 & -5.6 & 3.6 & -3.86 & .003 \\
Total Score & 1.4 & 50.4 & 7.8 & 4.6 & 3.84 & .003 \\
\hline
\end{tabular}

a two-tailed $p$-values were used, as the goal was to determine whether there was any difference between the two groups 


\section{Pre-Interview and Post-Interview}

The pre-interviews consisted of four parts: background questions, SBST questions, physical objects, and the complex figure. Open coding was performed on the background questions of the pre-interviews in order to get a robust history of the students. Students were asked about the types of toys they played with growing up and the sports they played. These answers were broken down into high spatial needs and low spatial needs categories. Toys such as blocks and puzzles are considered high spatial needs toys due to the need for the child to envision a structure in his or her mind, or manipulate a puzzle piece to determine where it should be placed. Sports such as football and soccer are considered high spatial needs sports because the athlete must track a ball in flight or analyze the path of another athlete in order to meet them at the ideal position. A summary of student responses can be seen in Table 6. This data was collected in part as an icebreaker activity in the pre-interview; therefore, further analysis was not performed on this data.

\section{Table 6}

Childhood Toys and Sports Participation by Level of Spatial Needs

\begin{tabular}{|c|c|c|c|c|}
\hline \multirow{2}{*}{$\begin{array}{l}\text { Level of Spatial Needs } \\
\text { High }\end{array}$} & \multicolumn{2}{|c|}{ Childhood Toys } & \multicolumn{2}{|c|}{ Activities/Sports } \\
\hline & $\begin{array}{l}\text { blocks } \\
\text { Legos }\end{array}$ & $\begin{array}{l}\text { puzzles } \\
\text { shape sorting }\end{array}$ & $\begin{array}{l}\text { baseball } \\
\text { basketball } \\
\text { football } \\
\text { gymnastics } \\
\text { marching band }\end{array}$ & $\begin{array}{l}\text { soccer } \\
\text { softball } \\
\text { tennis } \\
\text { volleyball }\end{array}$ \\
\hline Low & $\begin{array}{l}\text { action cards } \\
\text { action figures } \\
\text { chess } \\
\text { coloring } \\
\text { computers } \\
\text { dolls } \\
\text { electric kits } \\
\text { logic puzzles }\end{array}$ & $\begin{array}{l}\text { model cars } \\
\text { science kits } \\
\text { strategy games } \\
\text { stuffed animals } \\
\text { tv } \\
\text { video games } \\
\text { word puzzles }\end{array}$ & track & \\
\hline
\end{tabular}


In order to determine what spatial skills students used during the interview, the remaining student explanations were analyzed using deductive coding based on the Spatial Skills Framework developed by Engelke et al. (2016). Figure 11 presents a breakdown of the codes found in the pre-interviews. The split between two-dimensional and three-dimensional codes was fairly even. This is expected, as the interview tasks required both two-dimensional and threedimensional thinking: two-dimensional when considering cross-sections and their properties; three-dimensional when considering the objects as a whole.

\section{Figure 11}

Spatial Skills Framework Codes in the Pre-Interviews

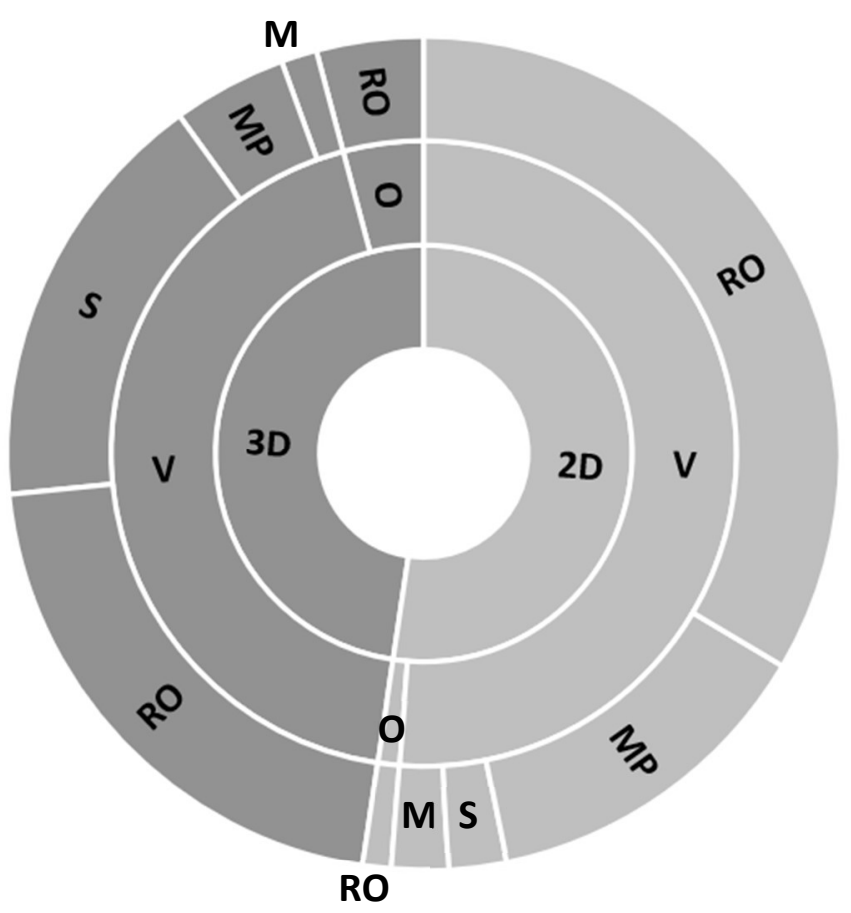

2D - two-dimensional

3D - three-dimensional

$\mathrm{O}$ - orientation

$\mathrm{V}$ - visualization

MP - mathematical

properties

$\mathrm{M}$ - measurement

RO - representing objects

$\mathrm{S}-$ structuring 
The tasks in the interviews relied heavily on visualization skills, so it is no surprise that almost 95 percent of the spatial language coded was visualization language. All of the orientation language was in the subcategory representing objects. In the two-dimensional realm, students sometimes used orientation when discussing the cross-sections for the SBST questions. For example, when Gwen was trying to decide between the correct answer and the egocentric answer, she said, "if you're looking at it head-on it's going to be right in front of you and it's not going to be tilted at all'. In this case, Gwen positioned herself (in her mind's eye) in front of the shape, as opposed to imagining the shape opening up to her (that would fall under visualization).

Three-dimensional orientation was demonstrated more often than two-dimensional orientation, though still not often. In the SBST portion of the pre-interview, James mentioned taking a "birds-eye view" of the shape when trying to distinguish between answer choices. More often, students demonstrated orientation language when attempting to construct the complex figure. As students struggled to draw meaningful three-dimensional representations, they sometimes resorted to drawing different viewpoints. Such was the case with Heidi, who described Figure 12 saying "this is like the top view if you're looking down on it".

\section{Figure 12}

The "Top-View" of Heidi's Complex Figure

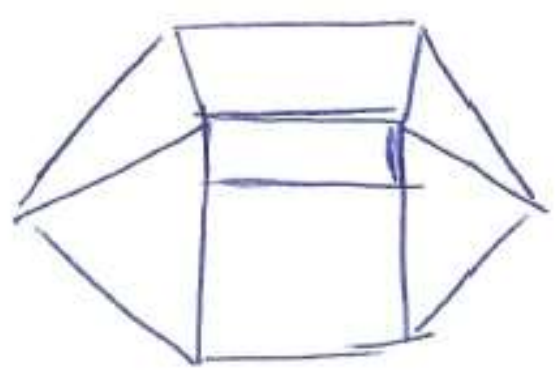


All four subcategories of visualization were demonstrated during the pre-interviews, with an emphasis on representing objects. This was to be expected, as the first two parts of the interview focused on finding the cross-sections of objects, in which the student would have to mentally manipulate the object to envision the cutting of a slice, and the last part of the interview focused on constructing a model of a three-dimensional object. Table 7 gives some examples of language that demonstrated spatial ability in the visualization, representing objects subcategories in two or three dimensions.

\section{Table 7}

Examples of Visualization-Representing Objects Spatial Language in Two and Three Dimensions

\begin{tabular}{|c|c|c|}
\hline Student & Quote & Context \\
\hline Monica & $\begin{array}{l}\text { Two-Dimensional } \\
\text { "Because it's cutting at a diagonal angle it would } \\
\text { create an elongated shape" }\end{array}$ & SBST question 27 \\
\hline Marcus & $\begin{array}{l}\text { "There's a slight inward curve right on it because } \\
\text { you look at it this curves in on here" }\end{array}$ & $\begin{array}{l}\text { Cross-section of the sponge with a } \\
\text { vertical cutting plane }\end{array}$ \\
\hline James & "It looks kind of like a donut" & $\begin{array}{l}\text { Cross-section of the duct tape with } \\
\text { a horizontal cutting plane }\end{array}$ \\
\hline \multicolumn{3}{|c|}{ Three-Dimensional } \\
\hline James & $\begin{array}{l}\text { "When you cut it diagonally down if you look at it in } \\
\text { the mirror it's going to show that diagonal cut } \\
\text { and then that cut will show an oval" }\end{array}$ & SBST question 10 \\
\hline Daniel & $\begin{array}{l}\text { "You have a hexagonal pure prism, and then you } \\
\text { stack a rectangle on top of it with um so that the } \\
\text { width and the length are equal to each other" }\end{array}$ & Complex figure \\
\hline Mary & $\begin{array}{l}\text { "There was an equilateral hexagon like on the top of } \\
\text { the figure. Then drop lines down from each and } \\
\text { then make like a square, and then the bottom } \\
\text { would also be a hexagon." }\end{array}$ & Complex figure \\
\hline
\end{tabular}


Measurement was the least utilized visualization-based spatial skill in the course of the pre-interviews. The students were not asked to make any calculations, but they were asked to consider how to find the areas of cross-sections when deciding which cutting plane should be used in order to find the volume of a physical object. Thus, the measurement language that was used was primarily in two dimensions, discussing how an area would theoretically be calculated or what measurements would be needed in order to determine an area. Daniel demonstrated both two-dimensional and three-dimensional measurement language. He began in two dimensions, saying, "you could find the radius and find the area of one circle and then find the area of the middle and then subtract them" and expanded to three dimensions as he continued, "and then just multiply that by however many."

The mathematical properties spatial ability was used in both the SBST and complex figure portions of the pre-interview. Students generally used mathematical properties language when they were describing the three-dimensional shape that was presented in the SBST questions and its relation to the cross-sections given as answer choices. For example, Daniel eliminated answer choice B for SBST question 10 when he recognized that "since it's a cone and it's like circular you know right off of the bat that it can't be like the half circular". In the complex figure portion of the pre-interview, two-dimensional mathematical properties came into play as students attempted to form the desired cross sections. Understanding the properties of the hexagon and square were crucial to success. As James began to work this problem, he said “there's supposed to be a hexagon, so you have to create six sides". This gave him a good base to begin constructing his three-dimensional figure. As Marcus attempted to form square crosssections, he said "to have a square, you'd have to have the base length the same as the height". 
This idea was critical to the formation of the complex figure, as he recognized that the height of the figure would have to change with the width of the base.

The primary purpose of the complex figure activity was to examine the students' structuring abilities. The nature of the activity requires the student to consider the described cross-sections as partitions of the three-dimensional figure. Gwen demonstrated this perfectly when she said "if I had like all of those piled up on top of each other and that's like the base then I could have hexagons [as horizontal cross-sections]". With this idea, Gwen was able to construct a hexagonal prism, as in Figure 13. There was also some two-dimensional structuring ability used during the physical objects portion of the interview, as students determined which way the object should be sliced in order to compute its volume. This ability was generally used to break down a cross-section for calculation of its area, as Mary described the cross-section of the sponge saying, "you could split this up into a triangle or a rectangle and two semi circles".

\section{Figure 13}

Gwen Formed a Hexagonal Prism by Stacking Hexagons

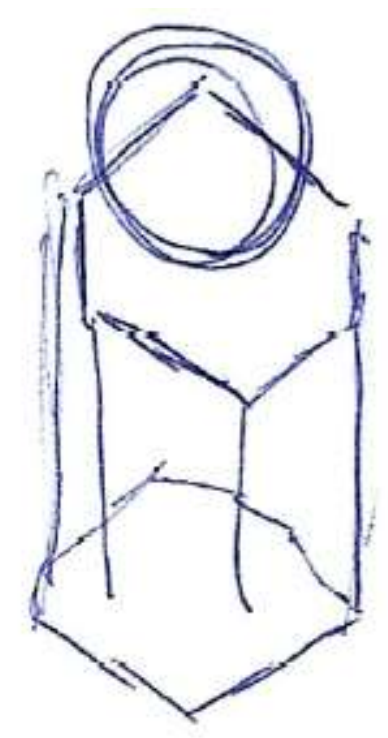


The post-interview consisted of the same four parts as the pre-interview, except that background questions were replaced with feedback on the intervention applications. Open coding was done to mark the feedback as positive, negative, or a specific suggestion for improvement. Several students said they enjoyed working with the intervention, that it was simple and easy to use, and that the concepts were interesting. Heidi found the activities stimulating, to the point that she said, "I found myself thinking about the objects a lot after I left". One student noted issues with the visual representation, especially when utilizing the camera movement to see the entire object. Another student commented that he would have preferred the cutting planes be shown one at a time instead of both on the same screen. Finally, one student felt like there was too-large a gap between the "easy" objects and the "hard" objects. No further analysis was done on this portion of the interview; the intervention feedback was collected as a reference for improving upon the work in the future.

Though the number of overall codes identified in the post-interviews was less than the number of codes identified in the pre-interviews, the distribution of the codes was very similar. This makes sense, as the interview protocol was nearly identical for the two interviews, and students could use fewer words to describe things that they saw and thought about before. The differences in the pre- and post-interviews include the icebreaker questions being replaced with questions to provide feedback on the intervention applications and a change in the requirements for the complex figure. As can be seen in Figure 14, the split between two-dimensional and three-dimensional spatial language was again nearly even; unlike the pre-interviews, threedimensional language was slightly more prominent than two-dimensional language in the postinterviews. 


\section{Figure 14}

Spatial Skills Framework Codes in the Post-Interviews

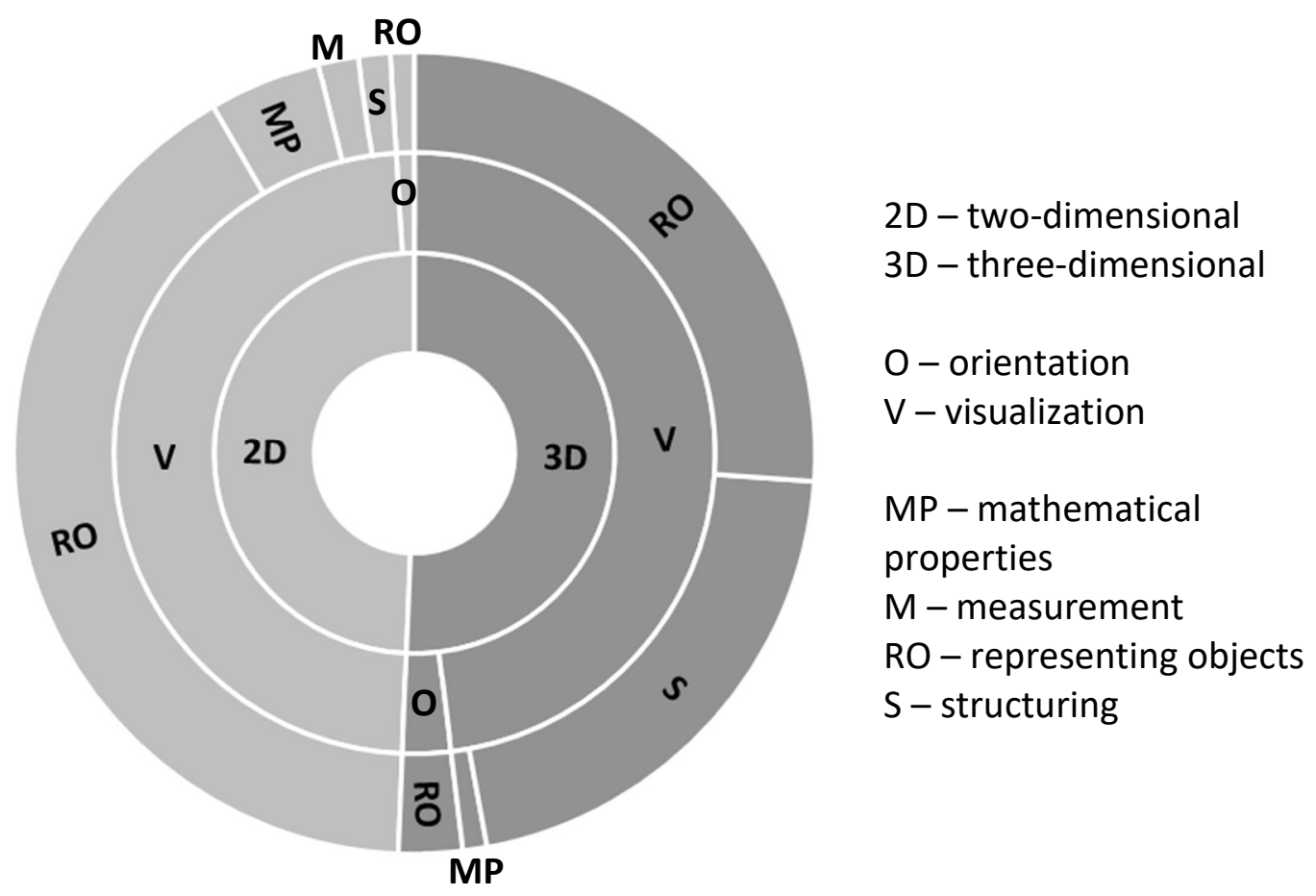

As in the pre-interviews, there was more visualization language than orientation language in the post-interviews (approximately 97 percent of the codes were in visualization), and all the orientation language used was in the representing objects subcategory. All of the language coded as two-dimensional, orientation, representing objects was demonstrated during the SBST portion of the post-interview. Monica and James both used the phrase "facing it head-on" to describe the mental picture of orienting themselves in front of the cross-section. Three-dimensional orientation language was again most prominent during the complex figure task, and was mostly used when drawing the object from different vantage points.

For both two-dimensional and three-dimensional visualization, representing objects was still the most demonstrated spatial skill. As stated earlier, this is somewhat expected, as the 
interview tasks ask the students to construct and describe cross-sections and a three-dimensional figure. There did not seem to be a tangible difference in the way students spoke about the objects they were representing, so it is not beneficial to provide more examples here; the primary takeaway is that the amount this skill was demonstrated was on par with the pre-interview.

Measurement was once again the least demonstrated visualization skill, and in the postinterview, there were actually zero cases of three-dimensional measurement on display. Similar to representing objects, the amount and content of structuring language was nearly identical to the pre-interviews; the few instances of two-dimensional structuring abilities were demonstrated by breaking a cross-section down to calculate its area, and the majority of the three-dimensional structuring abilities were centered around the complex figure activity.

The primary difference between the pre-interview and the post-interview coding results was in the mathematical properties subcategory. In the pre-interviews, the mathematical properties subcategory was on display nearly as much as the structuring subcategory, with the majority of instances in the two-dimensional realm. In the post-interviews, however, these numbers decreased drastically, despite the nearly identical protocols.

The spatial skills framework code application was also broken down by group to examine the differences between the Haptic and Non-Haptic Groups during the interviews. The results can be seen in Table 8 . As can be seen, the Non-Haptic Group consistently had more code applications in each category of the framework than the Haptic Group (with a few exceptions). This does not necessarily mean they possessed more advanced spatial abilities, but more than likely has to do with the amount each student spoke during the interview. In particular, the NonHaptic Group had a student who was very verbose in his explanations, which resulted in many more opportunities for using spatial language, and likely skewed the number of codes. 


\section{Table 8}

Spatial Skills Framework Codes by Group and Interview

\begin{tabular}{|c|c|c|c|c|}
\hline \multirow[t]{2}{*}{ Code } & \multicolumn{2}{|c|}{ Non-Haptic Group } & \multicolumn{2}{|c|}{ Haptic Group } \\
\hline & $\begin{array}{c}\text { Pre- } \\
\text { Interview }\end{array}$ & $\begin{array}{c}\text { Post- } \\
\text { Interview }\end{array}$ & $\begin{array}{c}\text { Pre- } \\
\text { Interview }\end{array}$ & $\begin{array}{c}\text { Post- } \\
\text { Interview }\end{array}$ \\
\hline $2 \mathrm{D}$ & 115 & 82 & 62 & 68 \\
\hline Orientation & 4 & & 1 & 3 \\
\hline $\begin{array}{l}\text { Mathematical Properties } \\
\text { Measurement }\end{array}$ & & & & \\
\hline Representing Objects & 3 & & 1 & 3 \\
\hline Structuring & & & & \\
\hline Visualization & 111 & 82 & 61 & 65 \\
\hline Mathematical Properties & 28 & 10 & 20 & 4 \\
\hline Measurement & 6 & 5 & 2 & \\
\hline Representing Objects & 80 & 67 & 41 & 60 \\
\hline Structuring & 6 & 2 & 2 & 2 \\
\hline $3 \mathrm{D}$ & 98 & 89 & 80 & 61 \\
\hline Orientation & 13 & 4 & 13 & 5 \\
\hline Mathematical Properties & & & & \\
\hline Measurement & & & & \\
\hline Representing Objects & 7 & 3 & 8 & 5 \\
\hline Structuring & & & & \\
\hline Visualization & 86 & 85 & 67 & 56 \\
\hline Mathematical Properties & 5 & 1 & 11 & 2 \\
\hline Measurement & 1 & & 4 & \\
\hline Representing Objects & 45 & 53 & 31 & 28 \\
\hline Structuring & 36 & 36 & 24 & 29 \\
\hline
\end{tabular}

Because of this, it makes more sense to compare each group to itself instead of comparing the groups to each other. Specifically, comparing the post-interview to the preinterview, the Non-Haptic Group used fewer amounts of each category of spatial language except for three-dimensional visualization - representing objects, and three-dimensional visualization - structuring. Similarly, the Haptic Group used more spatial language in the categories of two-dimensional orientation - representing objects, two-dimensional visualization - representing objects, and three-dimensional visualization - structuring.

In analyzing the student drawings for the pre- and post-interviews, a couple of misconceptions were identified and held by multiple students in the groups. The first 
misconception was that a curve in one plane of a physical object would result in the cross-section perpendicular to that plane also being curved. For example, when cutting a roll of duct tape perpendicular to the curve as in Figure 15, several students curved the edges of their rectangle and squares, as Heidi did in Figure 16. Though the curve of the edges tended to be subtle, Heidi confirmed the presence of the curved edges on the sponge cross-sections, saying "they would have a bit of curve, I suppose, because this kind of has a curve there".

\section{Figure 15}

Cutting a Roll of Duct Tape Perpendicular to the Curve
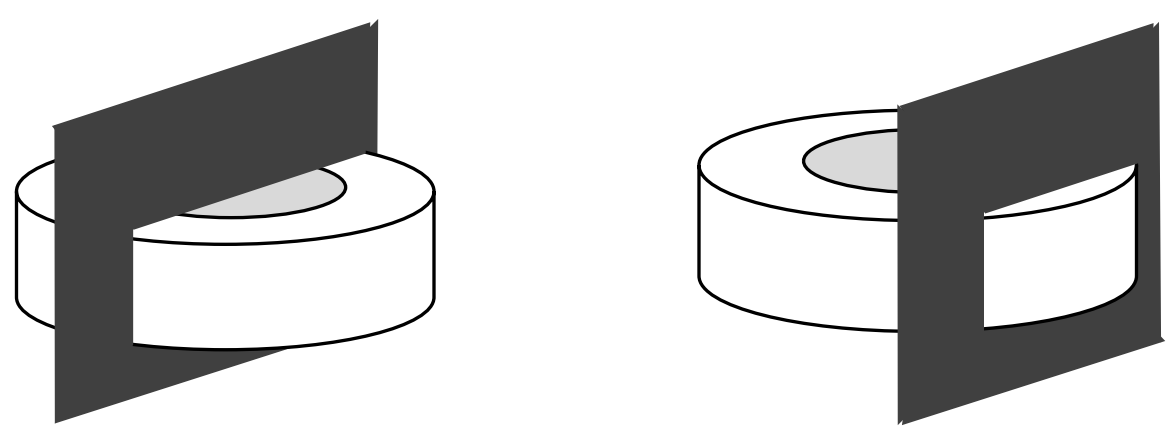

\section{Figure 16}

The Misconception That a Curve in One Plane of a Figure Results in Curved Cross-Sections

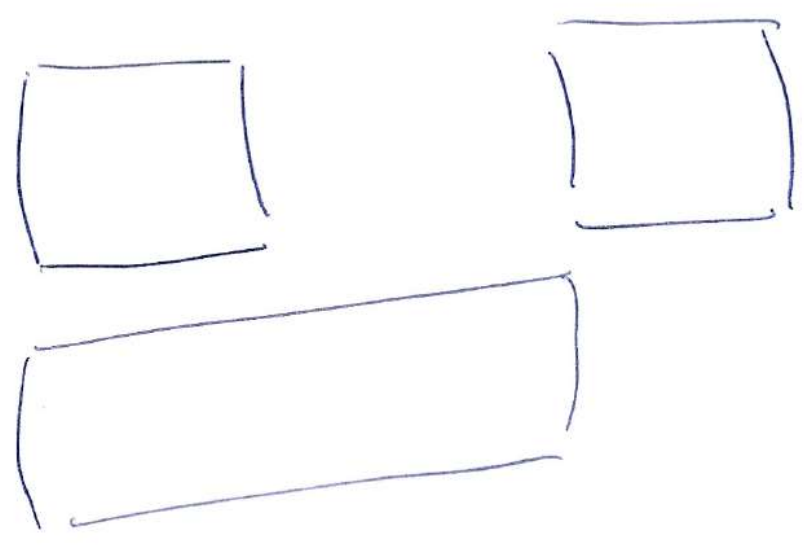


This same misconception was present in the cross sections of the sponge. The shape of the sponge curved from wide at the ends to narrower in the middle (see Figure 17), but the cross sections formed by a vertical cutting plane were all rectangles. Gwen and Heidi had the same misconception of the curved sides in both the pre-interview and the post-interview. Mary and Marcus had the misconception in the pre-interview, but not the post-interview.

Oddly, Daniel and James, both members of the Haptic Group, did not have the misconception in the pre-interview, but did present it in the post-interview. This could be evidence of an unintentional side-effect of the haptic intervention. Because of the manner in which the Novint Falcon is used, it can be difficult to discern between a rounded and a square edge. This is a distinction that is easier made after much experience working the device, but the students' limited use may have led them to believe edges of some figures were rounded when they were in fact square.

\section{Figure 17}

The Shape of the Sponge

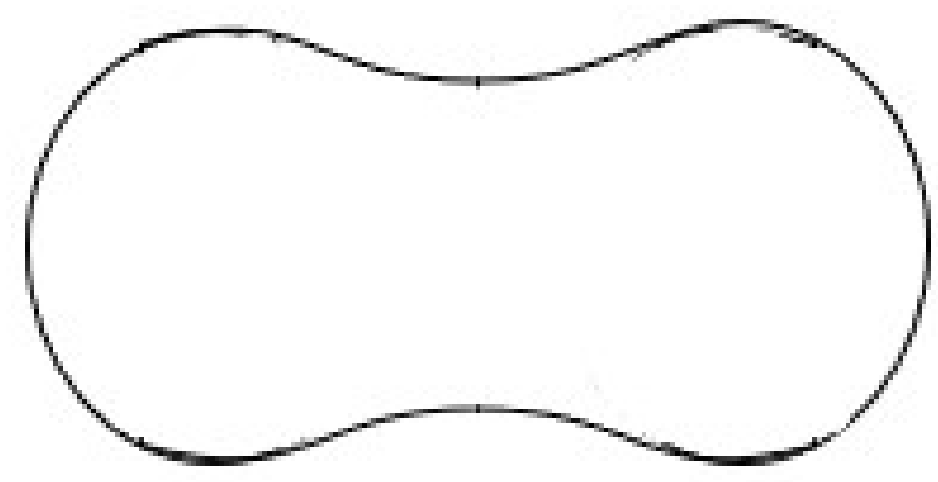


The other misconception brought forth by the physical objects portion of the interviews was the idea that a cross-section could look three-dimensional. This can be seen in Figure 18, as James sketched the back portion of the sponge and duct tape when asked to draw the cross section created as the result of the cutting plane similar to those in Figure 15. Daniel and Monica both showed this misconception in the pre-interviews, while James was the lone student to show it in the post-interviews.

While the drawings for the physical objects portion of the interview were fairly consistent amongst all the students, the complex figure portion of the interview resulted in a wide array of drawings. In the pre-interview, the students were asked to draw a figure with the following properties: most, but not all, cross-sections determined by a horizontal cutting plane are hexagons and all cross-sections determined by a vertical cutting plane are squares. Nearly every student was able to recognize that a hexagonal prism would yield all hexagonal cross-sections in one direction. Thus, every student except Abel drew something similar to what Gwen drew in Figure 13. Abel and Heidi were the only two who were able to construct a figure that had two different types of cross-sections when it was cut horizontally.

\section{Figure 18}

The Misconception That a Cross-Section is Three-Dimensional
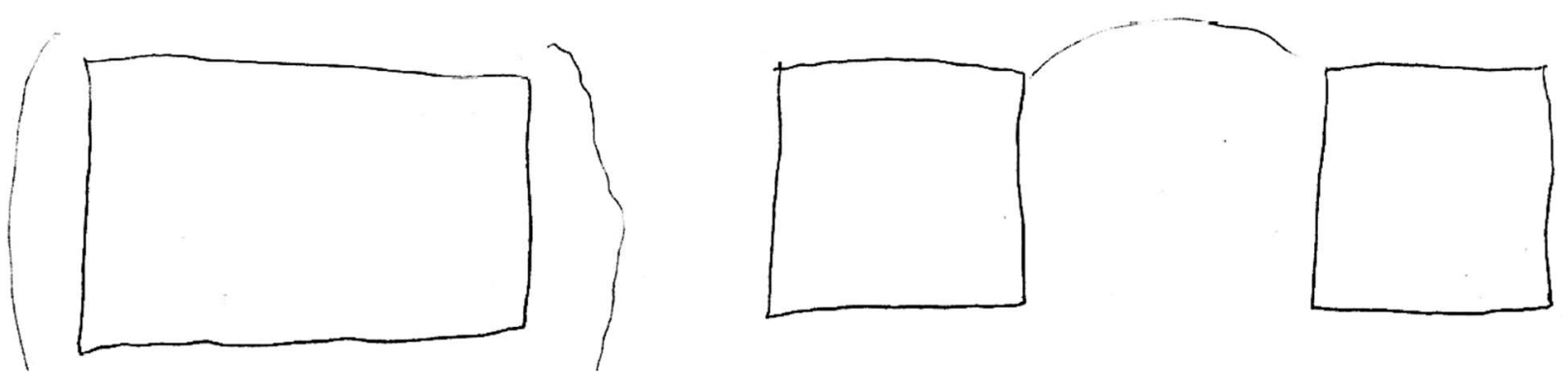
The biggest struggle for the students in the pre-interview task was determining how to construct squares for the vertical cross-sections. Most of the students recognized that the hexagonal prism could be constructed to have a square cross-section at each end and rectangular cross sections in between, but were unable to envision an object that changed height to match the width of the base. Heidi came the closest of all the students to creating a cohesive figure that met all of the expectations set forth in the problem. Table 9 presents the most cohesive drawing put together by each student for the complex figures portion of the pre-interview. 
Table 9

Complex Figures From the Pre-Interviews

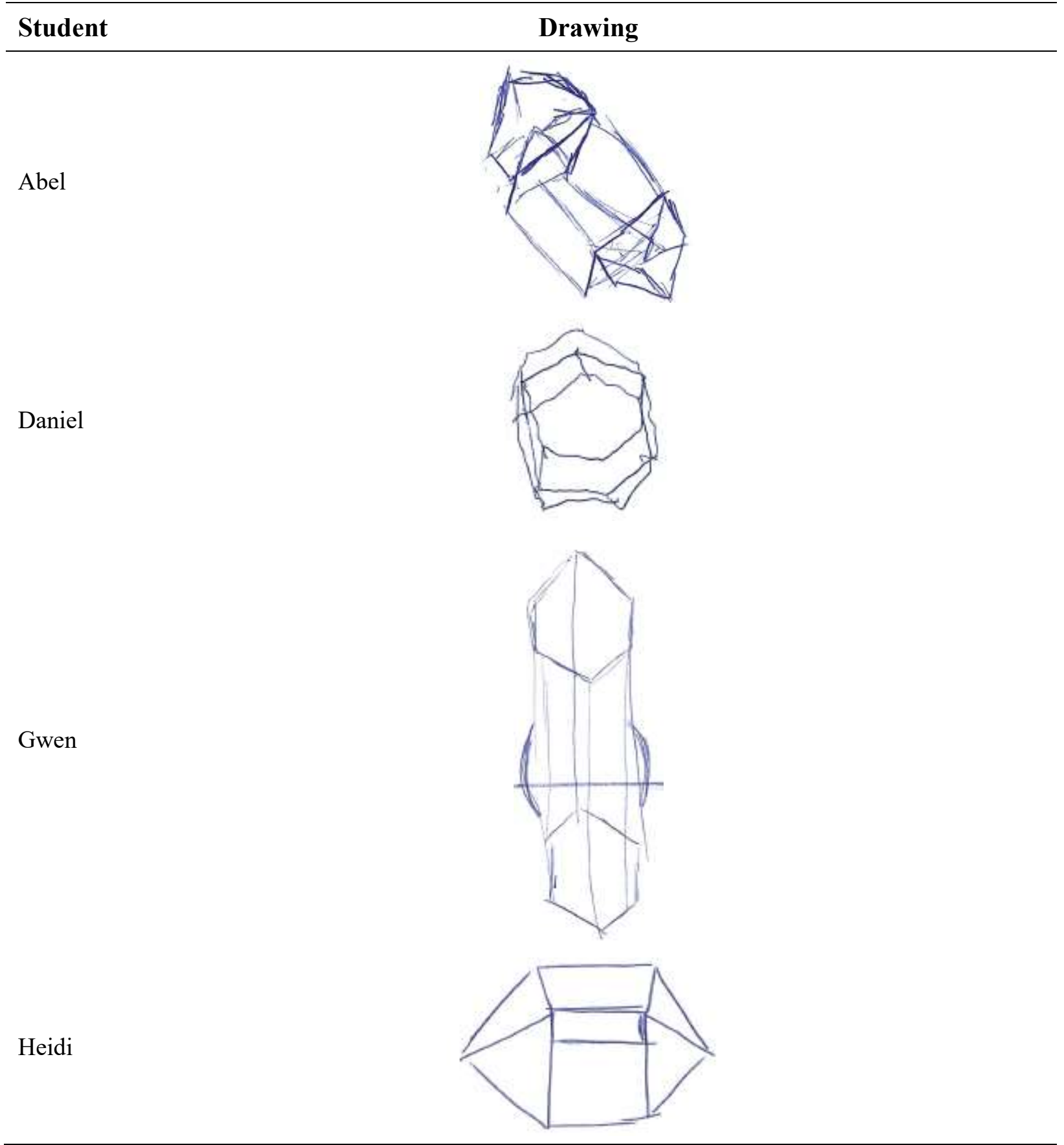




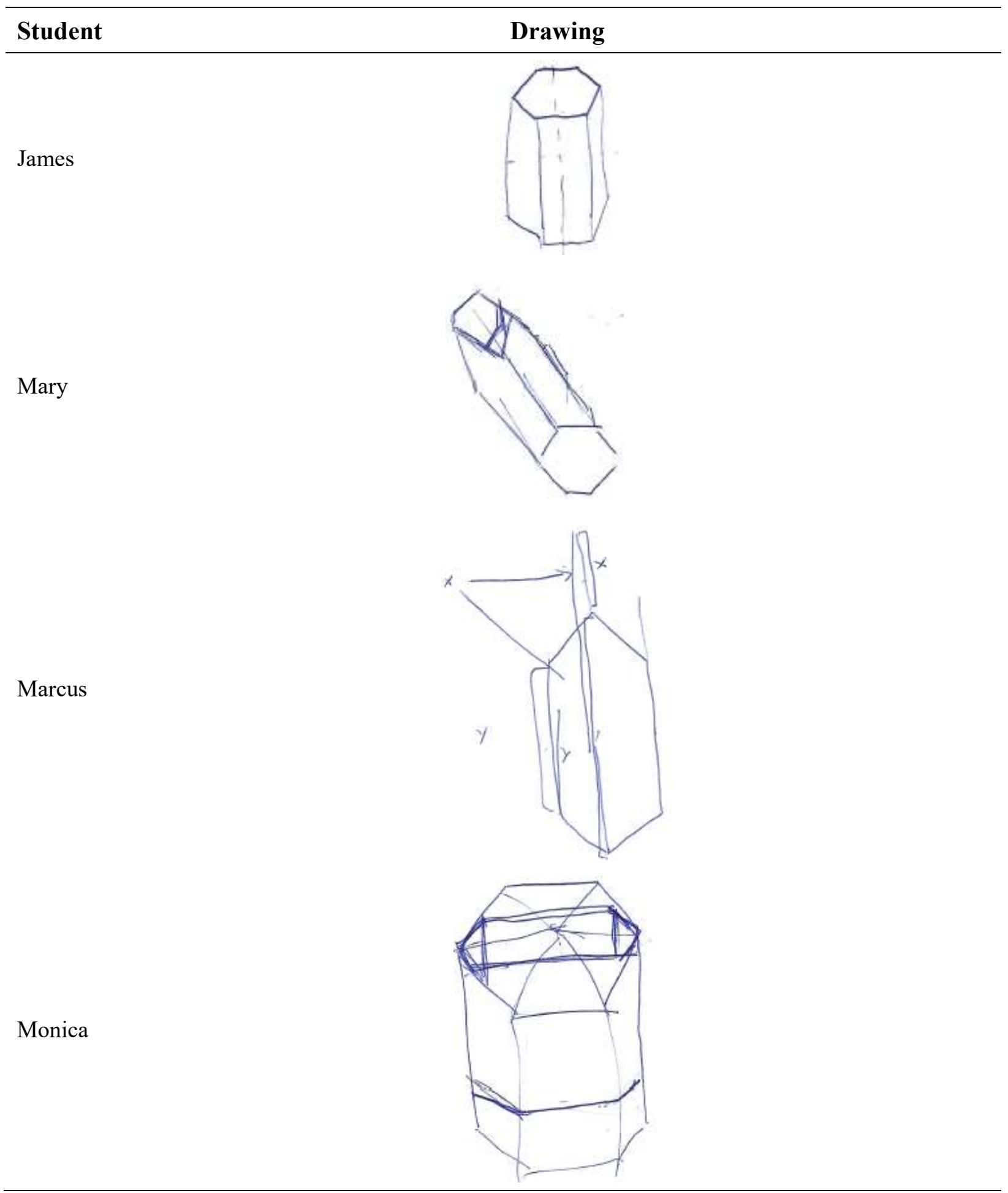

Note. Students were asked to draw a figure with the following properties: most, but not all, crosssections determined by a horizontal cutting plane are hexagons and all cross-sections determined by a vertical cutting plane are squares 
In the post-interview, the students were asked to draw a figure with the following properties: all, cross-sections determined by a horizontal cutting plane are pentagons and most, but not all, cross-sections determined by a vertical cutting plane are equilateral triangles. Like in the pre-interviews, all students were able to construct an object for the complex figure that had all pentagon cross-sections when cut with a horizontal cutting plane. Again, the primary difficulty students had was determining how to construct equilateral triangles when the size of the base was changing. Table 10 presents the drawings created by the students in the postinterviews. Monica attempted to break her figure into top, front, and side views in order to provide clarity of the objects shape. Of all the students, Monica was the only student to construct a figure that met all of the desired criteria. 
Table 10

Complex Figures From the Post-Interviews

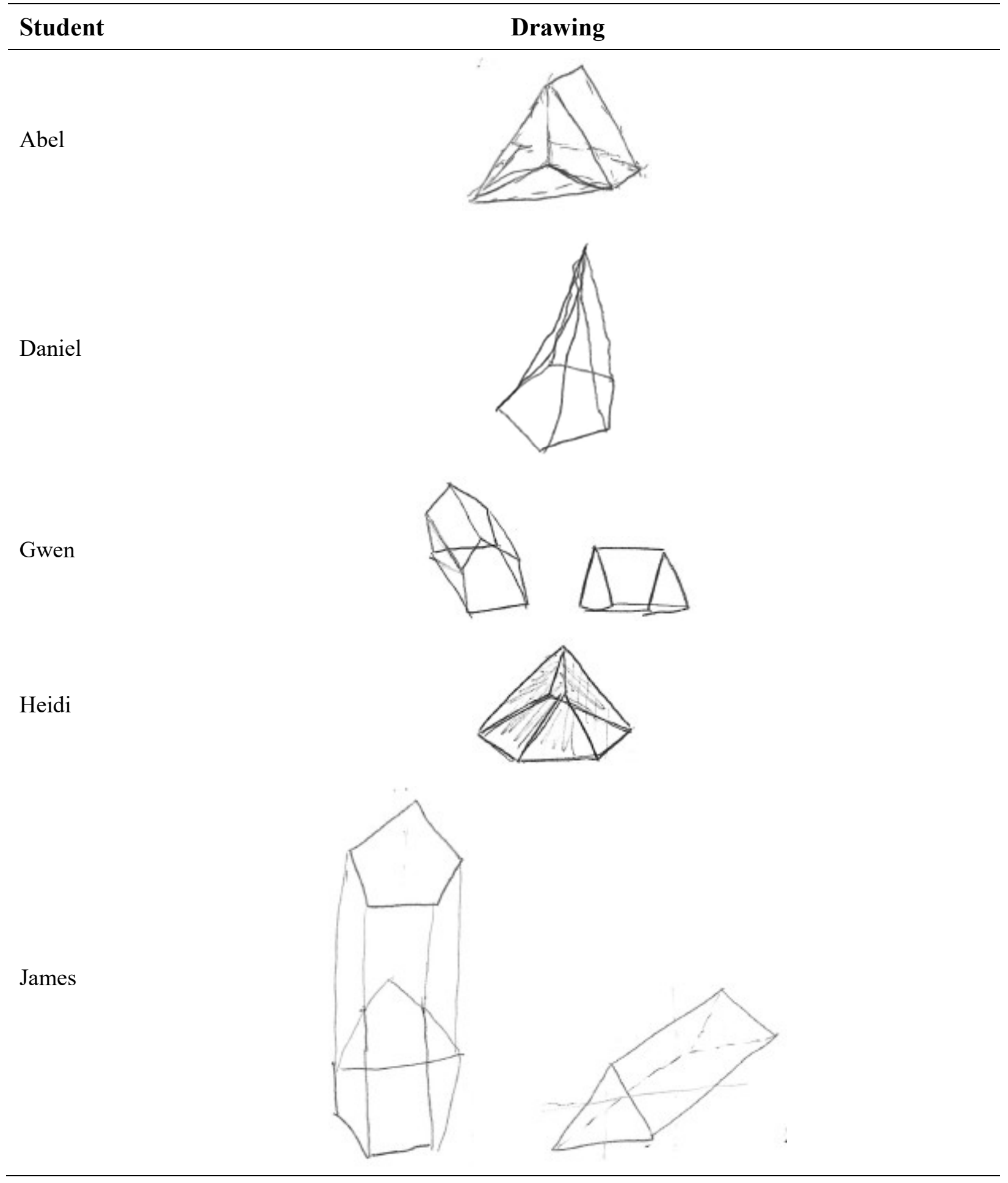




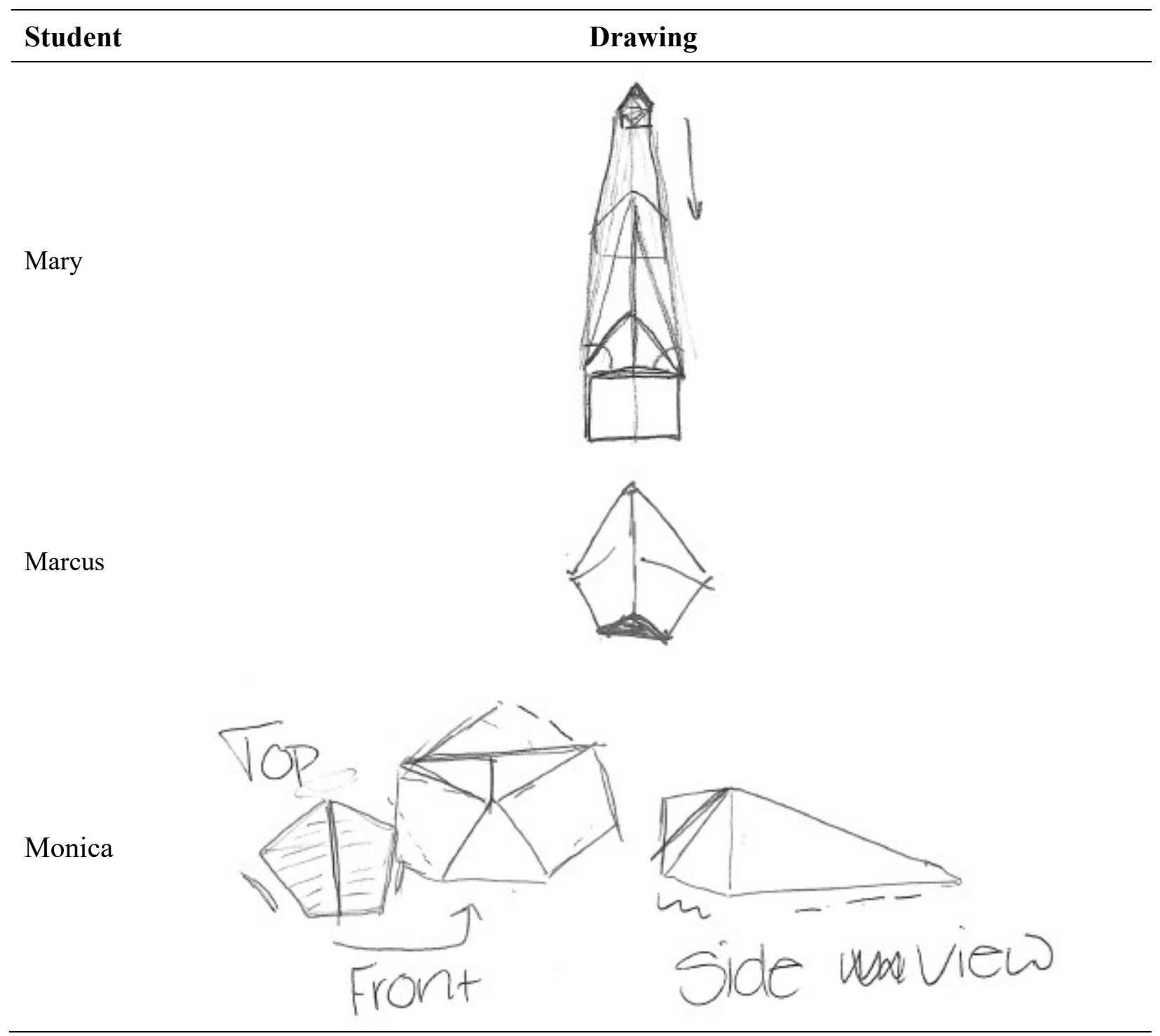

Note: Students were asked to draw a figure with the following properties: all, cross-sections determined by a horizontal cutting plane are pentagons and most, but not all, cross-sections determined by a vertical cutting plane are equilateral triangles. 
Similar to the analysis of the pre-test and post-test scores, paired two-sample $t$-tests were done on the interview scores in each group and the Interview Population to determine the significance of change from the pre-interview to the post-interview. The average scores and $t$-test results can be seen in Table 11. One-tailed $p$-values were used, as the expectation was that the score in each category and the total score would increase from pre-interview to post-interview.

\section{Table 11}

Paired Two-Sample t-Tests for Pre-Interview and Post-Interview Scores, with One-Tailed pValues

\begin{tabular}{|c|c|c|c|c|c|c|c|}
\hline \multirow[t]{2}{*}{ Group } & \multirow[t]{2}{*}{$n$} & \multicolumn{2}{|c|}{ Pre-interview } & \multicolumn{2}{|c|}{ Post-interview } & \multirow[t]{2}{*}{$t$} & \multirow[t]{2}{*}{$p^{\mathbf{a}}$} \\
\hline & & $\mathbf{M}$ & SD & $\mathbf{M}$ & SD & & \\
\hline \multicolumn{8}{|c|}{ SBST Questions } \\
\hline Non-Haptic Group & 4 & 4.8 & 1.0 & 4.5 & 1.3 & -0.26 & .404 \\
\hline Haptic Group & 4 & 3.8 & 1.7 & 4.5 & 1.3 & 1.57 & .108 \\
\hline Interview Population & 8 & 4.3 & 1.4 & 4.5 & 1.2 & -0.48 & .325 \\
\hline \multicolumn{8}{|c|}{ Physical Objects } \\
\hline Non-Haptic Group & 4 & 9.5 & 1.9 & 11.0 & 2.0 & 1.57 & .108 \\
\hline Haptic Group & 4 & 10.5 & 1.7 & 10.0 & 1.8 & -0.58 & .302 \\
\hline Interview Population & 8 & 10.0 & 1.8 & 10.5 & 1.9 & 0.71 & .251 \\
\hline \multicolumn{8}{|c|}{ Complex Figure } \\
\hline Non-Haptic Group & 4 & 3.5 & 0.6 & 4.3 & 0.5 & 3.00 & .023 \\
\hline Haptic Group & 4 & 3.8 & 1.3 & 4.0 & 1.6 & 0.40 & .359 \\
\hline Interview Population & 8 & 3.6 & 0.9 & 4.1 & 1.1 & 1.53 & .085 \\
\hline \multicolumn{8}{|c|}{ Total Score } \\
\hline Non-Haptic Group & 4 & 17.8 & 3.1 & 19.8 & 1.5 & 1.07 & .182 \\
\hline Haptic Group & 4 & 18.0 & 2.2 & 18.5 & 3.8 & 0.42 & .351 \\
\hline Interview Population & 8 & 17.9 & 2.5 & 19.1 & 2.7 & 1.17 & .139 \\
\hline
\end{tabular}

${ }^{\text {a }}$ one-tailed $p$-values were used, as the expectation was that the interview score would increase from pre-interview to post-interview 
Paired two-sample $t$-tests were also run on the matched pairs between the Non-Haptic and Haptic Groups for the pre-interview scores, post-interview scores and change scores in order to determine whether the haptic feedback played a significant role in improving spatial reasoning skills. The average values for each group as well as the two-tailed $p$-value as determined by each $t$-test can be seen in Table 12. Two-tailed $p$-values were used, as the goal was to determine whether there was any difference between the two groups.

\section{Table 12}

Paired Two-Sample t-Tests for Matched Pairs (n=4), with Two-Tailed p-Values

\begin{tabular}{|c|c|c|c|c|c|c|}
\hline \multirow[t]{2}{*}{ Section } & \multicolumn{2}{|c|}{ Non-Haptic Group } & \multicolumn{2}{|c|}{ Haptic Group } & \multirow[t]{2}{*}{$t$} & \multirow[t]{2}{*}{$p^{\mathrm{a}}$} \\
\hline & $\mathbf{M}$ & SD & M & SD & & \\
\hline \multicolumn{7}{|c|}{ Pre-Interview } \\
\hline SBST & 4.8 & 1.0 & 3.8 & 1.7 & -0.93 & .423 \\
\hline Physical objects & 9.5 & 1.9 & 10.5 & 1.7 & 1.41 & .252 \\
\hline Complex figure & 3.5 & 0.6 & 3.8 & 1.3 & 0.29 & .789 \\
\hline Total score & 17.8 & 3.1 & 18.0 & 2.2 & 0.12 & .909 \\
\hline \multicolumn{7}{|c|}{ Post-Interview } \\
\hline SBST & 4.5 & 1.3 & 4.5 & 1.3 & 0.00 & 1.000 \\
\hline Physical objects & 11 & 2.0 & 10.0 & 1.8 & -0.56 & .613 \\
\hline Complex figure & 4.3 & 0.5 & 4.0 & 1.6 & -0.24 & .824 \\
\hline Total score & 19.8 & 1.5 & 18.5 & 3.8 & -0.51 & .647 \\
\hline \multicolumn{7}{|c|}{ Change Score } \\
\hline SBST & -0.3 & 1.9 & 0.8 & 1.0 & 0.82 & .474 \\
\hline Physical objects & 1.5 & 1.9 & -0.5 & 1.7 & -1.85 & .161 \\
\hline Complex figure & 0.8 & 0.5 & 0.3 & 1.3 & -0.77 & .495 \\
\hline Total score & 2.0 & 3.7 & 0.5 & 2.4 & -0.79 & .486 \\
\hline
\end{tabular}

${ }^{a}$ two-tailed $p$-values were used, as the goal was to determine whether there was any difference between the two groups 
The scores of the Interview Population as a whole increased for each task as well as the total score, but none are considered to have increased significantly. The only score that demonstrated a significant change was the Non-Haptic Group's score for the complex figure task. Similar to the comparison of the SBST pre-test scores, the pre-interview scores did not show any significant difference between the groups. Again, this is expected as the two groups were set up as matched pairs. The post-interview scores and the change scores, however, did not follow the expectation that there would be a significant difference between the groups. It is likely that the range of possible scores and the small sample size prevent these statistics from being informative. 


\section{Discussion}

As stated earlier, the goal of the dissertation research was to determine what spatial abilities students possess when entering calculus, whether those spatial abilities that are underdeveloped (if any) can be remediated, and if so, whether haptic technology can play a role in promoting those spatial abilities. In this section, the research questions are answered using the data analysis from the previous section, and conclusions are drawn based on the answers to the questions.

\section{Research Question 1: What Spatial Skills Related to Cross-Sections Do Students Entering} Calculus Possess?

An examination of the SBST pre-test scores shows that students entering calculus possess some spatial skills relating to cross-sections, but there is definitely room for improvement. Adding together the average number of correct and egocentric answers shows that on average, students identified 25 of the 30 cross-sections correctly, but approximately 20 percent of the time, they did not orient themselves to the cross-section, thus resulting in the egocentric answer being chosen. This demonstrates that many students possess strong spatial visualization skills, but require improvement to their spatial orientation skills.

Furthermore, comparing the scores of the Below-Average Population to the Total Population we see that the average number of correct answers is lower and the average number of egocentric answers is higher. In fact, adding the average number of correct answers to the average number of egocentric answers identified by the Below-Average Population yields 22.7, just over 2 points below the Total Population average, despite the average total score being 8 below the Total Population average. This implies that the students identified as having lower 
spatial abilities based on the SBST pre-test scores primarily struggle in the area of spatial orientation.

The pre-interview results support this idea as well. Recall that the Interview Group and the Below-Average Group together make up the students in the Total Population who scored below the average on the SBST pre-test. Spatial orientation was the least demonstrated spatial skill during the pre-interviews. It was expected that this skill would be demonstrated less based on the nature of the interview activities, but the vast difference in the amount of visualization and orientation language used implies that students rely much more on their visualization skills. Because knowledge is constructed from experience, this would imply that students entering calculus with under-developed spatial skills simply do not have much experience in using spatial orientation skills.

The pre-interviews also demonstrated students' difficulties in the structuring subcategory. The complex figure task was designed specifically to test students' structuring abilities in the visualization skill. The task gives the students the partitions to begin with, and the student simply has to use his or her visualization and structuring skills to form them into a cohesive object. The simplest piece of this problem was the horizontal cross-sections, as there was no restriction based on being a regular figure. Most of the students possessed some structuring ability, as they were able to create a prism to satisfy the condition. The addition of the vertical cross-sections, however, lead to great struggles, as the students could not accommodate the changing height into their figure.

An area that was demonstrated strongly by the students in the pre-interviews was the representing objects skill. Over half the spatial language used fell into this category in some manner (in both two and three dimensions, in both orientation and visualization). Again, this 
language was certainly expected, as the pre-interview tasks do rely heavily on the representing objects ability, but the sheer dominance indicates that students are very comfortable in this realm. This is likely due to the fact that students are consistently required to represent objects in mathematics courses - whether they are drawing two-dimensional and three-dimensional shapes in geometry, or graphing lines in algebra, this is a very common spatial skill utilized in mathematics.

Based on the SBST pre-test scores and the pre-interviews, students entering calculus possess spatial visualization skills, specifically in the subcategory of representing objects. They also possess some orientation and structuring skills, but likely need to work to improve these skills. From the constructivist view, this means the students have likely had little exposure to activities requiring those skills, as knowledge is constructed from experiences. If a remediation course were to be put into place, an emphasis on these two skills would give students the experiences necessary to build upon their current knowledge and create a better foundation for these skills. Otherwise, instructors can help students build these skills by developing problems that utilize them. Recall that the constructivist view says these skills are based on experience; it is up to the instructor to push the students into experiences that will allow them to develop these skills.

\section{Research Question 2: What Difference in Performance Does the Haptic Group Exhibit Over the Non-Haptic Group in the Post-Interview?}

The analysis of the post-interview activity scores shows that there was not much difference in performance during the post-interview between the Haptic and Non-Haptic Groups. None of the individual sections of the interview, nor the overall score demonstrated a significant difference between the groups. As expected, there was not a significant difference between the 
groups in the pre-interview (this is the goal of a matched pair design, after all), but the combination of these two similarities results in the change scores being shown as not significantly different as well.

A comparison of the spatial language used in the post-interviews is not easily done because of the vast difference in the amount students verbalized during both the pre- and postinterviews. Specifically, Marcus had the longest post-interview and the second most codes applied because he was very outspoken and verbose. James, on the other hand, had the shortest interview and the fewest number of codes applied because he tended to give one-word answers, if he spoke at all; at times, he just drew the cross-section and offered no explanation, even when prompted. These differences could be attributed to differences in personality, confidence in the material, or comfort with the interviewer. Marcus was part of the Non-Haptic Group, while James was part of the Haptic Group. This lead naturally to the Non-Haptic Group having more code content than the Haptic Group.

Looking at the explanations students gave during the post-interviews, nothing stood out as a glaring difference between members of the Haptic Group and members of the Non-Haptic Group. One hypothesis is that the Haptic Group would demonstrate language related to haptics, such as imagining touching the object, in the post-interview as a result of being introduced to the haptic feedback. This was not the case. In fact, only one member of either group made a comment related to the interventions as he was working through the problems. When working on an SBST problem, Daniel said, "I wish, you know, like I could turn the cone around to see where the plane was actually, like, leaving". Daniel was a member of the Haptics Group, but the comment he made does not apply specifically to the haptic feedback or the additional functionality presented to the Haptic Group. Based on this, the conclusion is that there was no 
real difference in performance between the Haptic Group and the Non-Haptic Group in the postinterview. This implies that the use of the haptic intervention did not introduce new information for the student to accommodate into their existing schema.

\section{Research Question 3: How Do the Performance and Explanations Differ Among the Interview Population From the Pre-Interview to the Post-Interview?}

Similar to research question 2 , there is not much to say regarding the difference between the pre-interview and the post-interview for the Interview Population. The analysis of the preinterview and post-interview scores showed that there was no significant difference in scores, though the score of each portion did increase.

The amount of spatial language coded as a part of the spatial skills framework actually decreased from the pre-interview to the post-interview. The downward trend is likely due to a few factors. First, prior to the pre-interview, the students had limited work with cross-sections. Three of the eight students had never taken a calculus class. The remaining five students had taken a college or high school calculus class, but the fact that they were enrolled in the twosemester calculus course, generally reserved for students who need the material presented at a slower pace, is telling. This means that knowledge on cross-sections had not been constructed, or the knowledge that had been constructed was not robust, so the students spent the pre-interview trying to accommodate the new information into something meaningful or assimilate their existing notions to allow their new experiences to align with the old.

By the time the students participated in the post-interview, they had been working with cross-sections for four weeks (participating in the interventions) and had much more familiarity. Through this time, the students were able to bring new ideas on spatial reasoning into their knowledge base, modifying the existing structures as necessary. The fact that the students' 
knowledge constructs were more robust may have caused their answers to be more concise, and therefore contain less spatial language.

Another potential reason for the lower number of instances of spatial language is that the SBST questions and physical objects used were the same for both interviews. The interviews were spaced only five weeks apart, making it likely that the students could remember the preinterview tasks and give answers from memory instead of having to work the problems. In this case, the students would not use much spatial language, if they used any language at all. Again, the exposure to these specific tasks in the pre-interviews allowed the students to construct a knowledge base that included these specific cases. Then, during the post-interviews, instead of being forced into an act of assimilation or accommodation because of new information, they are able to draw on their existing constructs.

\section{Research Question 4: How Does the Difference in the Pre/Post Spatial Skills Test Given at the Beginning and the End of the Semester Differ Among the Groups?}

An examination of the material presented in the first semester of the two-semester Calculus I course showed that although two-dimensional spatial reasoning skills are employed, there is little work done in the three-dimensional realm. Because of this, a significant improvement in spatial skills was not expected in students as a result of attending the class. This was the case for the General Population, as none of the improvements made are considered significant. Looking at the Below-Average population, however, shows that the number of correct answers identified and the total score both increased significantly. This implies that simply participating in the course work is enough to promote spatial skills in students who enter calculus with some of these skills under-developed. 
These results are supported by a constructivist point of view, because the students in the Below-Average Population likely did not have strong constructs for spatial skills upon entering the course, and would be forced to assimilate or accommodate the new spatial ideas into their existing constructs as they are presented during the course. The remaining students in the General Population (those who scored above average on the SBST pre-test), however, did not likely have to make as many accommodations of new knowledge, because the new knowledge and existing knowledge would align, and thus would not show much change in ability.

The Interview Population showed highly significant improvements in all three types of scores (correct answers, egocentric answers, total score). These improvements are more significant than the improvements in the Below-Average Population, leading to the conclusion that the intervention activities were successful in promoting an even greater increase in spatial abilities than simply going through the calculus course.

This conclusion is backed by the results of the two-sample $t$-tests with unequal variance. Comparing the General Population to the Interview Population, there were very significant differences in all three scores of the pre-test, with each $p$-value .002 or less. This indicates that the Interview Population performed significantly different, and in this case worse, than the General Population. Looking at the post-test scores, however, shows that the Interview Population did not score significantly different than the General Population. So, the Interview Population was significantly different than the General Population prior to working through the intervention activities, but was not significantly different after. This means that participating in the intervention activities allowed students who performed significantly worse than their peers on the pre-test to improve their abilities to the level of their peers on the post-test. 
The $t$-tests comparing the Below-Average Population to the Interview Population yield similar results, but in the opposite direction. Prior to the intervention activities, the BelowAverage Population and the Interview Population were not significantly different; all three scores for the pre-test had $p$-values indicating this. On the post-test, however, the Interview Population scored significantly better on the Number of Correct answers and the Total Score. Essentially, the intervention activities were able to raise the scores of the students in the Interview Population to the level of the General Population.

Finally, examining the change scores for the Interview Population compared to both the General Population and the Below-Average Population show that the Interview Population improved its scores significantly over each of the other populations. Caution must be used when looking at change scores for the General Population, as the higher the score on the pre-test, the less room for improvement on the post-test, thus resulting in a low change score. The BelowAverage Population, however, had plenty of room for improvement, and arguably the same amount of room for improvement as the Interview Population, as both populations scored below the average on the SBST pre-test and there was no significant difference between their pre-test scores. This makes the comparison between the change scores valid, and further supports that the intervention activities did promote spatial skills in the students.

Next, the Haptic Group and Non-Haptic Group were compared to see if the use of haptics had any effect on the improvement. Looking at the paired two-sample $t$-tests on the pre- and post-test scores, the change for each group is considered significant, and the one-tailed $p$-values are similar, so it is not easy to tell whether the haptics resulted in greater improvement. While observably the Haptic Group had a larger change, the $p$-values for these $t$-tests all showed that the differences between the groups were not significant for the post-test or for the change in 
scores from the pre-test to the post-test. This indicates that the haptics part of the intervention did not result in significant gains on the spatial skills of the students. The small size of the matched group (four students per group) may have contributed to this finding.

\section{Conclusions}

Overall, the dissertation research shows that some students possess under-developed spatial skills when they arrive in calculus. In particular, spatial orientation skills and structuring skills are less developed than spatial visualization and representing objects skills. The research also shows that although participating in the calculus course may somewhat increase orientation skills, a better alternative is to put students through spatial skills remediation, as it was shown that a simple four-week intervention was able to significantly increase the SBST scores.

An interesting point regarding the intervention is that the students did not receive feedback during the course of the activities - they simply worked through the problems to the best of their abilities. This course of action allowed them to build on the knowledge they already had and accommodate the new knowledge in their own way. This is also important to note because remediation with students who have under-developed spatial skills can be done with minimal effort from the instructor - simply creating the activities and having the students walk through them on their own is enough to promote development.

Finally, this research is inconclusive with regard to whether haptics can be used to further promote spatial skills in first semester calculus students. While slightly higher gains were observed when the intervention included haptic technology, these gains were not significant. This does not, however, rule out further testing using haptics to assist with improving spatial skills. These results are not necessarily generalizable due to the small number of matched pairs. 


\section{Teaching Implications}

Based on this research, it is highly recommended that some sort of remediation of spatial skills be performed with undergraduate mathematics students, specifically during calculus courses. This remediation could be done as a stand-alone course, individual tasks outside of the normal coursework, or tasks integrated into the course.

Although some have had success in implementing a stand-alone remediation course, such as Sorby et al., (2013), this idea may present logistical difficulties as it requires a full course worth of material, a physical location (unless the course is designed as an online course), and someone to lead the course. If these are successfully established, the next difficulty is filling the course with students. If the course is not required, many students may choose not to take it, whether due to already having a full course load, not understating the need for the course, or simply not wanting to add another course. Requiring the course for certain students may be possible, but would involve further planning and approvals.

Providing additional tasks outside of the normal coursework would remove some of the obstacles that creating a course presents - there is no need for a separate instructor and meeting time, no approvals would be necessary with regard to making the course a requirement, and no overhead would be required. The amount of material required would likely be less, as it wouldn't have to fill an entire course of content. If the additional material is not presented as a requirement, however, the same difficulties exist regarding student participation. Requiring the work without overloading the instructor is a possibility, as the research showed that spatial skills are increased simply by performing the tasks, even if no feedback is given. However, if students realize the tasks are not being graded for correctness, the effort put forth may diminish. 
Therefore, the ultimate recommendation for instructors is to incorporate spatial reasoning tasks into the course itself. One way to do this is to push students to create the three-dimensional drawings that represent the solids of revolution for which they are computing the volume.

Though it is entirely possible for these types of problems to be performed without seeing the full three-dimensional shape, the ability to envision the result of the revolution can greatly help with the decision of which method to use to determine the volume. It is highly recommended that instructors do not encourage students to work around their difficulties in drawing threedimensional figures by demonstrating alternative methods, but instead to allow them to practice this skill. Some time may even be devoted specifically to teaching students how to draw threedimensional figures that have been created by rotating two-dimensional figures.

Keeping in mind that spatial orientation is one of the more under-developed skills for these students, activities that specifically ask the student to utilize spatial orientation skills are recommended as well. These types of problems could be similar to those in the SBST, if focusing on volumes of solids of revolution. Some creativity could also be used to get students to use orientation skills in other parts of the curriculum, such as sketching derivatives. For example, the students may be asked to imagine themselves standing at a particular point of the graph, such as an extreme value, and look in each direction to determine the slope on each side of that point. Similarly, the student might be asked to imagine walking along the graph to determine the slope at each point. Both ideas require the student to change their orientation while performing the tasks.

Finally, it is recommended that attention be paid to developing structuring skills. There are several opportunities in calculus for these skills to be developed, such as constructing Riemann Sums for integrals and imagining the slices of a three-dimensional solid during the 
forming of the integral to represent its volume. Problems similar to the "complex figure"

problem given during the interviews are another way to push the students to develop structuring skills. 


\section{Future Work}

There are several ways the research in this dissertation can be extended and improved in order to learn more about spatial skills and the potential for haptic technology to assist in their remediation.

To better determine whether haptics can be beneficial in the remediation of spatial skills, the research design can be scaled to include a larger Total Population, and perhaps more noteworthy, more matched pairs. The number of matched pairs for this research was kept somewhat small (it began with five pairs - one pair dropped out) due to the decision to perform pre- and post-interviews with the students and the coding and analysis of these interviews, which is quite time-consuming. As the interviews did not seem to shed much light on differences between the Haptic Group and the Non-Haptic Group, it could be beneficial to remove this part of the research design and simply perform a pre-test/post-test design with the intervention. This would allow for many more matched pairs to be included, thus giving a better dataset for analysis and the ability to generalize the results.

That said, the interview process was not futile. Performing the interviews with the students enabled the collection of a baseline set of spatial skills. In a future project, the protocol for the interviews could be improved to contain more problems similar to the complex figure problem, in that it pushes heavily on one particular spatial skill (three-dimensional visualization - structuring). By including more problems that focus on a specific skill, a more robust and accurate picture of the spatial skills students possess when entering calculus can be created. Another improvement to the interview protocol would be to use different physical objects in the post-interview than in the pre-interview. Because the same objects were used in both interviews, the students had a sense of familiarity, which may have allowed them to perform the tasks with 
more ease, thus preventing a clear understanding of how their spatial thinking may have changed from the pre-interview.

Finally, the properties of the complex figure used in the post-interview should be the exact same as those in the pre-interview, except for the actual shapes. This was an unintentional complication made in the protocol for the post-interview. The complex figure in the preinterview had a certain regular polygon for all cross-sections formed by vertical cutting planes. This forced the student to determine how to keep the shape regular while one side was changing length. It was specified that the cross-sections formed by horizontal cutting planes were mostly one shape, but it was required that not all the cross-sections be that shape. This was described to students as making sure at least one cutting plane resulted in a different cross-section than the rest. When the complex figure conditions were presented in the post-interview, the condition of all cross-sections versus mostly the same cross-sections was accidentally swapped, so that the vertical cross-sections resulted in mostly a regular polygon, but not all. This lessened the emphasis on the student having to account for a changing side length, because if the crosssection became irregular, the figure would still meet the criteria.

The intervention applications could also be improved for future research. Through the post-interviews and a short survey directly following the last application, the Interview Population gave their opinions on the intervention applications and made suggestions for improvements. One issue that was noted was that the rotation of the object could be glitchy. This was due to the camera moving around the object, instead of the object rotating in space. Another issue was that the haptic object did not always align perfectly with the visual object. These two issues could be remedied to make the applications more reliable. 
Some of the students in the Haptic Group admitted that they could have utilized the haptics device more, but were held back by unfamiliarity. This could be alleviated by giving them more time to work with the introductory application, or even more applications to work through, with the hope that by the end of the intervention period, they have reached a higher comfort level. There was no record of whether or how much students used the haptic device during the sessions, beyond self-reporting in the survey after the last application. In the future, measuring the time each student used the device, or even observing the use, could help to better understand how the student gained from its use. A few suggestions were also made to make the applications better fit the students' needs, such as only presenting one cutting plane to the user at a time (in the applications, two parallel planes were presented simultaneously) and including the ability for the user to see the true cross-section as a means of checking their work.

Another consideration for future work is to change the haptic controller to be used. The Novint Falcon was chosen for this project for several reasons. First, it was familiar to the researcher, as a previous internship and prior research had utilized the same controller. Second, it was a relatively low-cost haptics device (around $\$ 250$ ), which could reasonably be purchased by departments wanting to implement the application for remediation. Other haptic controllers, such as the SensAble Touch or Touch X, are very costly (from $\$ 2,500$ - $\$ 15,000$ ), which can put a strain on an academic department or prevent the department from purchasing multiple, or any, devices. Part of the purpose of this research is to encourage the formation of remedial courses to promote spatial skill development, so having an affordable option seemed necessary. Unfortunately, between the collection of data and the publication of this dissertation, Novint has stopped selling the Falcon. Although some devices are still available through a third-party vendor, development and support for the controller has ceased, meaning no new features are 
being created and any issues with existing features cannot be remedied. Because of this, it would be beneficial to examine other haptics devices and modify the applications to work with a new low-cost option.

Advances in virtual reality (VR) technology have made VR systems more readily available and much less expensive. This opens the possibility of performing the intervention activities using a VR system with haptic feedback. One such system is the Wireality, developed by Fang et al. (2020) at Carnegie Mellon University. This VR system allows the user to feel objects in the VR setting by providing force feedback through cables that attach to the fingertips, palm, and wrist with finger caps and Velcro straps. The authors estimate that the controller will only cost $\$ 50$ in volume production, so schools interested in implementing a remedial course could purchase several of these for student participants. One major drawback exists for this system, however. The authors found that the Wireality does not perform well when simulating flat surfaces or complex objects due to the force feedback being only in one direction. This problem would render the device useless for use in the intervention, but further development could result in a viable option for replacing the Falcon in a future study.

Finally, the same ideas from this research could be explored through other means of providing haptic feedback. The price of 3D printers has decreased and they are now being widely used in educational settings. Instead of providing haptic feedback in the intervention, a 3D printer could be used to create physical models. 


\section{References}

Albert, W. S., \& Golledge, R. G. (1999). The use of spatial cognitive abilities in geographical information systems: The map overlay operation. Transactions in GIS, 3(1), 7-21. https://doi.org/10.1111/1467-9671.00003

Applebee, D., Bennett-Day, B., Ferrari, J., Pritchard, P., \& Boettger-Tong, H. (2021). Multimodal training improves spatial reasoning skills in female college students. Journal of Science Education and Technology, 30, 539-549. https://doi.org/10.1007/s10956-02009898-6

Bivall, P., Ainsworth, S., \& Tibell, L. A. E. (2011). Do haptic representations help complex molecular learning? Science Education, 95(4), 700-719. https://doi.org/10.1002/sce.20439

Bodner, G. M., \& Guay, R. B. (1997). The Purdue visualization of rotations test. The Chemical Educator, 2(4), 1-17. https://doi.org/10.1007/s00897970138a

Braun, V., \& Clarke, V. (2006). Using thematic analysis in psychology. Qualitative Research in Psychology, 3(2), 77-101. https://doi.org/10.1191/1478088706qp063oa

Burte, H., Gardony, A. L., Hutton, A., \& Taylor, H. A. (2017). Think3d!: Improving mathematics learning through embodied spatial training. Cognitive Research: Principiles and Implications, 2(1), 13. https://doi.org/10.1186/s41235-017-0052-9

Carlisle, D., Tyson, J., \& Nieswandt, M. (2015). Fostering spatial skill acquisition by general chemistry students. Chemistry Education Research and Practice, 16, 478-517. https://doi.org/10.1039/c4rp00228h

Chellali, A., Dumas, C., \& Milleville-Pennel, I. (2012). Haptic communication to support biopsy procedures learning in virtual environments. Presence: Teleoperators and Virtual Environments, 21(4), 470-489. https://doi.org/10.1162/PRES_a_00128

Cohen, C. A., \& Hegarty, M. (2012). Inferring cross sections of 3D objects: A new spatial thinking test. Learning and Individual Differences, 22(6), 868-874. https://doi.org/10.1016/j.lindif.2012.05.007

Darrah, M. A. (2013). Computer haptics: A new way of increasing access and understanding of math and science for students who are blind and visually impaired. Journal of Blindness Innovation and Research, 3(2). https://doi.org/10.5241/3-47

Darrah, M., Murphy, K., Speransky, K., \& DeRoos, B. (2014). Framework for K-12 education haptic applications. IEEE Haptics Symposium (HAPTICS) (pp. 409-414). Houston, TX: IEEE. https://doi.org/10.1109/HAPTICS.2014.6775490

Dubinsky, E. (1991). Reflective abstraction in advanced mathematical thinking. In D. O. Tall (Ed.), Advanced Mathematical Thinking (pp. 95-126). Kluwer Academic Publishers. 
Engelke, N., Darrah, M., \& Murphy, K. (2016). A framework for examining the 2-D and 3-D spatial skills needed for calculus. In T. Fukawa-Connelly, N. Engelke Infante, M. Wawro, \& S. Brown (Ed.), Proceedings of the 19th Annual Conference on Research in Undergraduate Mathematics Education, (pp. 736-740). Pittsburgh, PA.

Fang, C., Zhang, Y., Dworman, M., \& Harrison, C. (2020). Wireality: enabling complex tangible goeometries in virtual reality with worn multi-string haptics. CHI '20: Proceedings of the 2020 CHI Conference on Human Factors in Computing Systems (pp. 1-10). Honolulu, HI: Association for Computing Machinery. https://doi.org/10.1145/3313831.3376470

Fiboni V.O.F. (n.d.). Spatial reasoning hard. Fibonicci: https://www.fibonicci.com/spatial-awareness/spatial-reasoning-test/hard/

Gorlewicz, J. L. (2013). The efficacy of surface haptics and force feedback in education. (Publication No. 3570810) [Doctoral dissertation, Vanderbilt University]. ProQuest Dissertations and Theses Global.

Hamza-Lup, F. G., \& Adams, M. (2008). Feel the pressure: E-learning systems with haptic feedback. 2008 Symposium on Haptic Interfaces for Virtual Environment and Teleoperator Systems, (pp. 445-450). Reno, NV. https://doi.org/10.1109/HAPTICS.2008.4479991

Hegarty, M., \& Waller, D. (2004). A dissociation between mental rotation and perspective-taking spatial abilities. Intelligence, 32(2), 175-191. https://doi.org/10.1016/j.intell.2003.12.001

Jones, M. G., Minogue, J., Oppewal, T., Cook, M. P., \& Broadwell, B. (2006). Visualizing without vision at the microscale: Students with visual impairments explore cells with touch. Journal of Science Education and Technology, 15(5), 345-351. https://doi.org/10.1007/s10956-006-9022-6

Jones, S. R. (2015). Areas, anti-derivatives, and adding up pieces: Definite integrals in pure mathematics and applied science contexts. The Journal of Mathematical Behavior, 38, 928. https://doi.org/10.1016/j.jmathb.2015.01.001

Jones, S. R. (2015). The prevalence of area-under-a-curve and anti-derivative conceptions over Riemann sum-based conceptions in students' explanations of definite integrals.

International Journal of Mathematical Education in Science and Technology, 46(5), 721736. https://doi.org/10.1080/0020739X.2014.1001454

Kersh, J., Casey, B. M., \& Young, J. M. (2008). Research on spatial skills and block building in girls and boys: The relationship to later mathematics learning. In O. N. Saracho, \& B. Spodek (Eds.), Contemporary perspectives on mathematics in early childhood education (pp. 233-252). Information Age Publishing Inc.

Kimura, D. (1999). Sex and cognition. MIT Press. 
Kurtulus, A. (2013). The effects of web-based interactive virtual tours on the development of prospective mathematics teachers' spatial skills. Computers \& Education, 63, 141-150. https://doi.org/10.1016/j.compedu.2012.11.009

Lawrence, M. M. (2011). Behavioral and neurological studies in tactile map use and training by persons who are blind or visually impaired. (Publication No. 3466366) [Doctoral dissertation, University of Oregon]. ProQuest Dissertations and Theses Global.

Lee, A. L., \& Wong, K. W. (2014). Learning with desktop virtual reality: Low spatial ability learners are more positively affected. Computers \& Education, 79, 49-58. https://doi.org/10.1016/j.compedu.2014.07.010

Lowrie, T., Logan, T., \& Hegarty, M. (2019). The influence of spatial visualization training on students' spatial reasoning and mathematics performance. Journal of Cognition and Development, 20(5), 729-751. https://doi.org/10.1080/15248372.2019.1653298

Martin-Dorta, N., Saorin, J. L., \& Contero, M. (2011). Web-based spatial training using handheld touch screen devices. Educational Technology \& Society, 14(3), 163-177.

Martin-Gutierrez, J., Saorin, J. L., Contero, M., Alcaniz, M., Perez-Lopez, D. C., \& Ortega, M. (2010). Design and validation of an augmented book for spatial abilities develompment in engineering students. Computers \& Graphics, 34(1), 77-91. https://doi.org/10.1016/j.cag/2009.11.003

McGee, M. G. (1979). Human spatial abilities: Psychometric studies and environmental, genetic, hormonal, and neurological influences. Psychological Bulletin, 86(5), 889-918. https://doi.org/10.1037/0033-2909.86.5.889

Meredith, D. C., \& Marrongelle, K. A. (2008). How students use mathematical resources in an electrostatics context. American Journal of Physics, 76(6), 570-578. https://doi.org/10.1119/1.2839558

Merriam-Webster. (n.d.). Ability. In Merriam-Webster.com dictionary. www.merriamwebster.com/dictionary/ability

Merriam-Webster. (n.d.). Haptic. In Merriam-Webster.com dictionary. www.merriamwebster.com/dictionary/haptic

Merriam-Webster. (n.d.). Skill. In Merriam-Webster.com dictionary. www.merriamwebster.com/dictionary/skill

Minogue, J., Jones, M. G., Broadwell, B., \& Oppewall, T. (2006). The impact of haptic augmentation on middle school students' conceptions of the animal cell. Virtual Reality, 10(3-4), 293-305. https://doi.org/10.1007/s10055-006-0052-4

Murphy, K., \& Darrah, M. (2015). Haptics-based apps for middle school students with visual impairments. IEEE Transactions on Haptics, 8(3), 318-326. https://doi.org/10.1109/TOH.2015.2401832 
National Research Council. (2012). Discipline-based education research: Understanding and improving learning in undergraduate science and engineering. (S. R. Singer, N. R. Nielsen, \& H. A. Schweingruber, Eds.) National Academies Press.

Okumus, S., \& Hollebrands, K. (2016). High school students' forming 3D objects using technological and non-technological tools. Proceedings of the Thirty-Eighth Annual Meeting of the North American Chapter of the International Group for the Psychology of Mathematics Education, (pp. 243-250). Tucson, AZ.

Persson, P. B., Cooper, M. D., Tibell, L. A. E., Ainsworth, S., Ynnerman, A., \& Jonsson, B.-H. (2007). Designing and evaluating a haptic system for biomolecular education. 2007 IEEE Virtual Reality Conference (pp. 171-178). Charlotte, NC: IEEE. https://doi.org/10.1109/VR.2007.352478

Piaget, J. (1970). Structuralism. Basic Books.

Pittalis, M., \& Christou, C. (2010). Types of reasoning in 3D geometry thinking and their relation with spatial ability. Educational Studies in Mathematics, 75(2), 191-212. https://doi.org/10.1007/s10649-010-9251-8

Prawat, R. S., \& Floden, R. E. (1994). Philosophical perspectives on constructivist views of learning. Educational Psychology, 29(1), 37-48. https://doi.org/10.1207/s15326985ep2901_4

Schoenfeld, A. H. (1987). Cognitive science and mathematics education: An overview. In A. H. Schoenfeld (Ed.), Cognitive Science and Mathematics Education (pp. 1-31). Lawrence Erlbaum Associates.

Sealey, V. (2006). Definite integrals, Riemann sums, and area under a curve: What is necessary and sufficient? In S. Alatorre, J. L. Cortina, M. Saiz, \& A. Mendez (Ed.), Proceedings of the Twenty Eighth Annual Meeting of the North American Chapter of the International Group for the Psychology of Mathematics Education. 2, pp. 46-53. Merida, Yucatan, Mexico: Universidad Pedagogica Nacional.

Sealey, V. (2014). A framework for characterizing student understanding of Riemann sums and definite integrals. The Journal of Mathematical Behavior, 33, 230-245. https://doi.org/10.1016/j.jmathb.2013.12.002

Shimomura, Y., Hyannberg, E. T., \& Hafsteinsson, H. (2013). Haptic cues as a utility to perceive and recognise geometry. Universal Access in the Information Society, 12(2), 125-142. https://doi.org/10.1007/s10209-012-0271-2

Simon, M. A. (1995). Reconstructing mathematics pedagogy from a constructivist perspective. Journal for Research in Mathematics Education, 26(2), 114-145. https://doi.org/10.2307/749205 
Sorby, S., Casey, B., Veurink, N., \& Dulaney, A. (2013). The role of spatial training in improving spatial and calculus performance in engineering students. Learning and Individual Differences, 26, 20-29. https://doi.org/10.1016/j.lindif.2013.03.010

Taylor, H. A., \& Hutton, A. (2013). Think3d!: Training spatial thinking fundamental to STEM education. Cognition and Instruction, 31(4), 434-455. https://doi.org/10.1080/07370008.2013.828727

Thompson, P. W., \& Silverman, J. (2008). The concept of accumulation in calculus. In M. P. Carlson, \& C. Rasmussen (Eds.), Making the connection: Research and teaching in undergraduate mathematics education (pp. 43-52). Mathematical Association of America. https://doi.org/10.5948/UPO9780883859759.005

Thurstone, L. L. (1969). Primary mental abilities (double page reprint ed.). University of Chicago Press.

Toennies, J. L., Burgner, J., Withrow, T. J., \& Webster, R. J. (2011). Toward haptic/aural touchscreen display of graphical mathematics for the education of blind students. 2011 IEEE World Haptics Conference (pp. 373-378). Istanbul, Turkey: IEEE. https://doi.org/10.1109/WHC.2011.5945515

Uttal, D. H., \& Cohen, C. A. (2012). Spatial thinking and STEM education: When, why, and how? In B. H. Ross (Ed.), Psychology of Learning and Motivation (Vol. 57, pp. 147181). Academic Press. https://doi.org/10.1016/B978-0-12-394293-7.00004-2

Xie, F., Zhang, L., Chen, X., \& Xin, Z. (2020). Is spatial ability related to mathematical ability: A meta-analysis. Educational Psychology Review, 32, 113-155. https://doi.org/10.1007/s10648-019-09496-y

Yoon, S. Y. (2011). Psychometric properties of the Revised Purdue Spatial Visualization Tests: Visualization of Rotations (the Revised PSVT:R). (Publication No. 3480934) [Doctoral dissertation, Purdue University]. ProQuest Dissertations Publishing.

Young, C. J., Levine, S. C., \& Mix, K. S. (2018). The connection between spatial and mathematical ability across development. Frontiers in Psychology, 9, 755. https://doi.org/10.3389/fpsyg.2018.00755 
Appendix A: Santa Barbara Solids Test 
Date:

ID:

\section{Cross Section Test}

This is a test about cross-sections. A cross-section is the $2 \mathrm{D}$ shape that results when a cutting plane intersects an object.

You've seen many examples of cross-sections in everyday life. For example, when you slice an apple from top to bottom, the resulting cut surface is a cross-section of the apple.

The picture below shows an apple with some worms inside. Note that the cross section on the right shows both the apple and the shapes and locations of the sliced worms inside the apple.

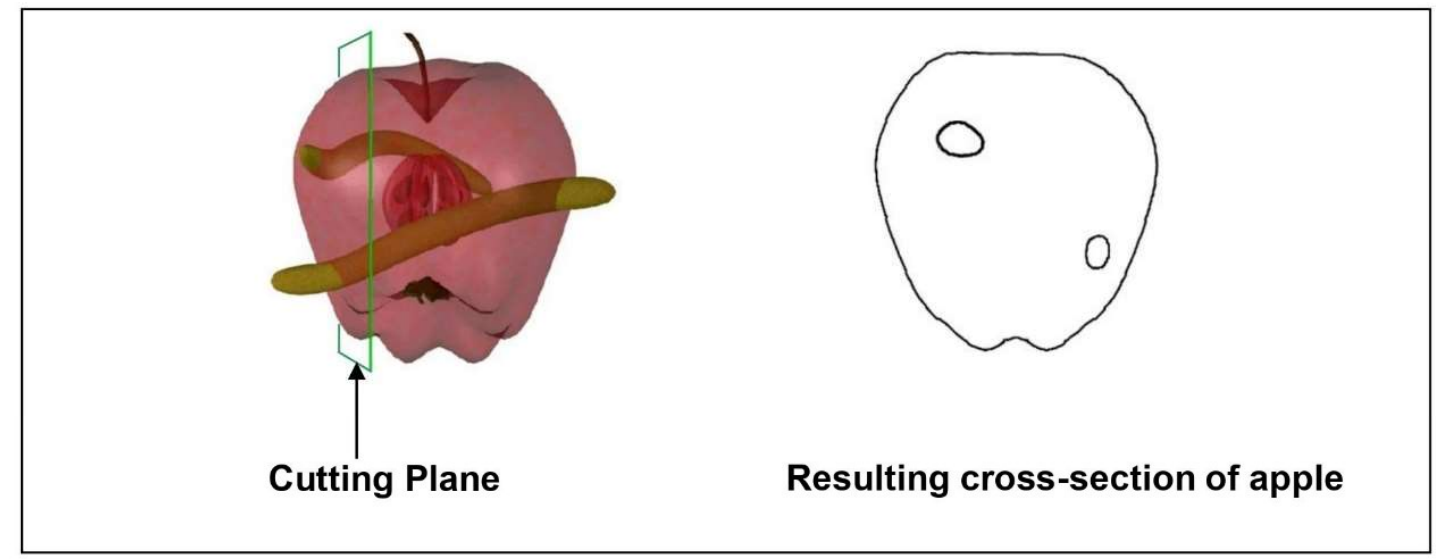


In this multiple choice test, you will be asked to identify the cross sections of three types of figures:

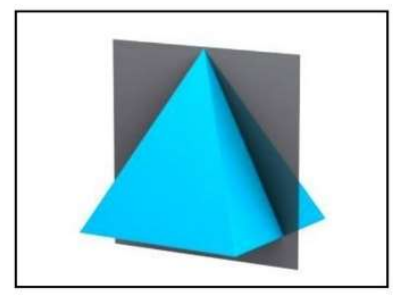

Single object

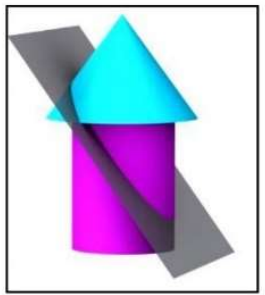

Attached objects

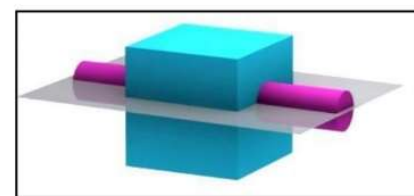

Nested objects (one object is inside another)

Here are some important things to remember:

- All figures are solid (not hollow) objects.

- The objects are about 6-8 inches tall. Imagine that they are on the table in front of you.

- Attached figures are "glued together" at their edges.

- Nested objects consist of one object inside another. In the nested object above, the cylinder extends all the way through the cube. If you sliced this figure, you would see the cylinder inside the cube.

The cutting planes, shown in grey, will have different orientations.

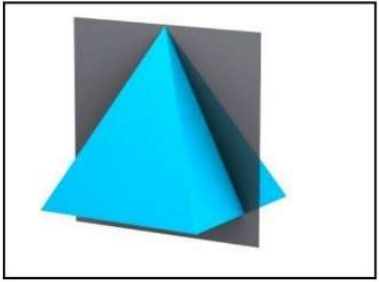

Vertical Plane

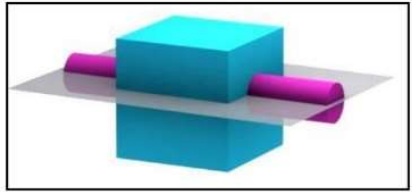

Horizontal Plane

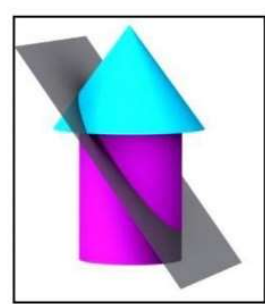

Oblique Plane 
You will see three types of cutting planes: horizontal, vertical, and oblique.

For each type of cutting plane, try to imagine the cross section that would result if you faced the cutting plane head-on, as if you were looking at your reflection in a mirror.

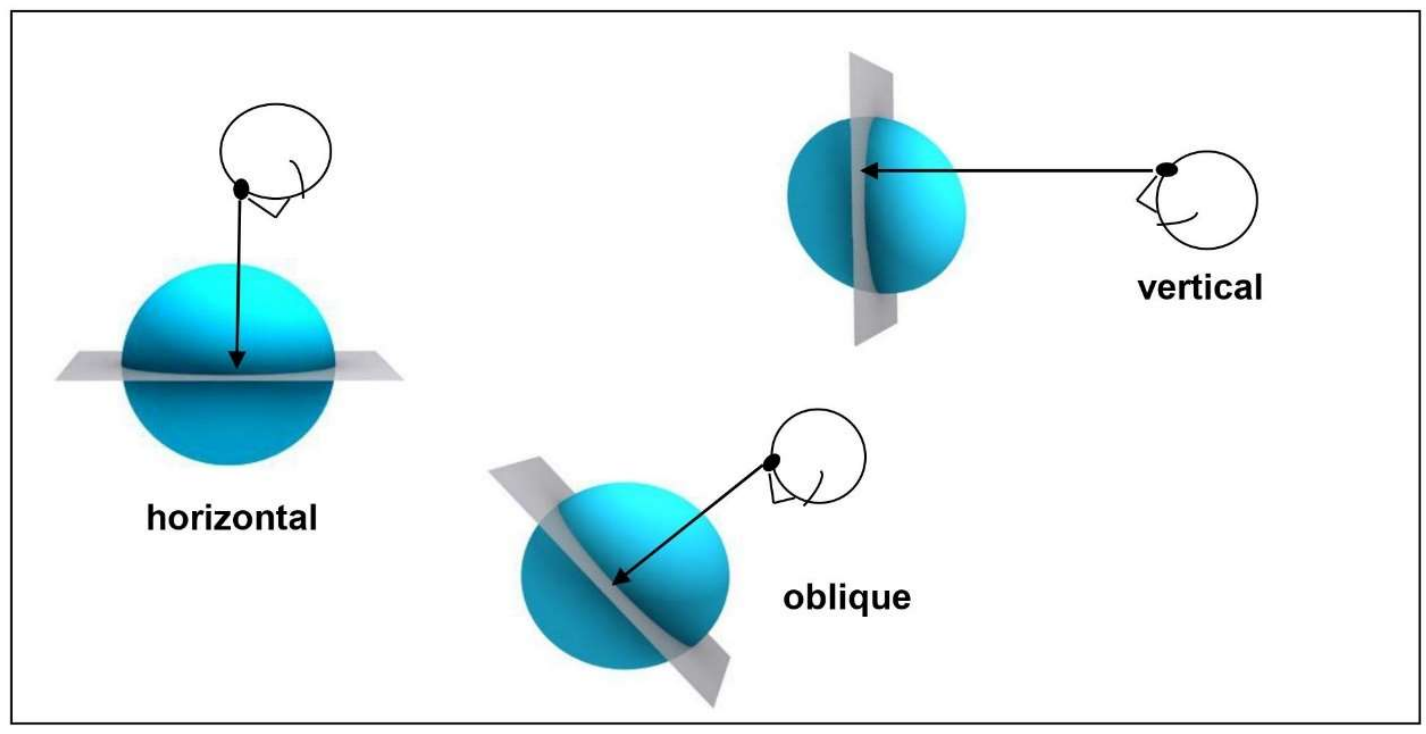

You should also assume that the objects are 6-8 inches tall, and that they are sitting on the desk in front of you.

In the example below, the cutting plane would produce the cross section on the right.

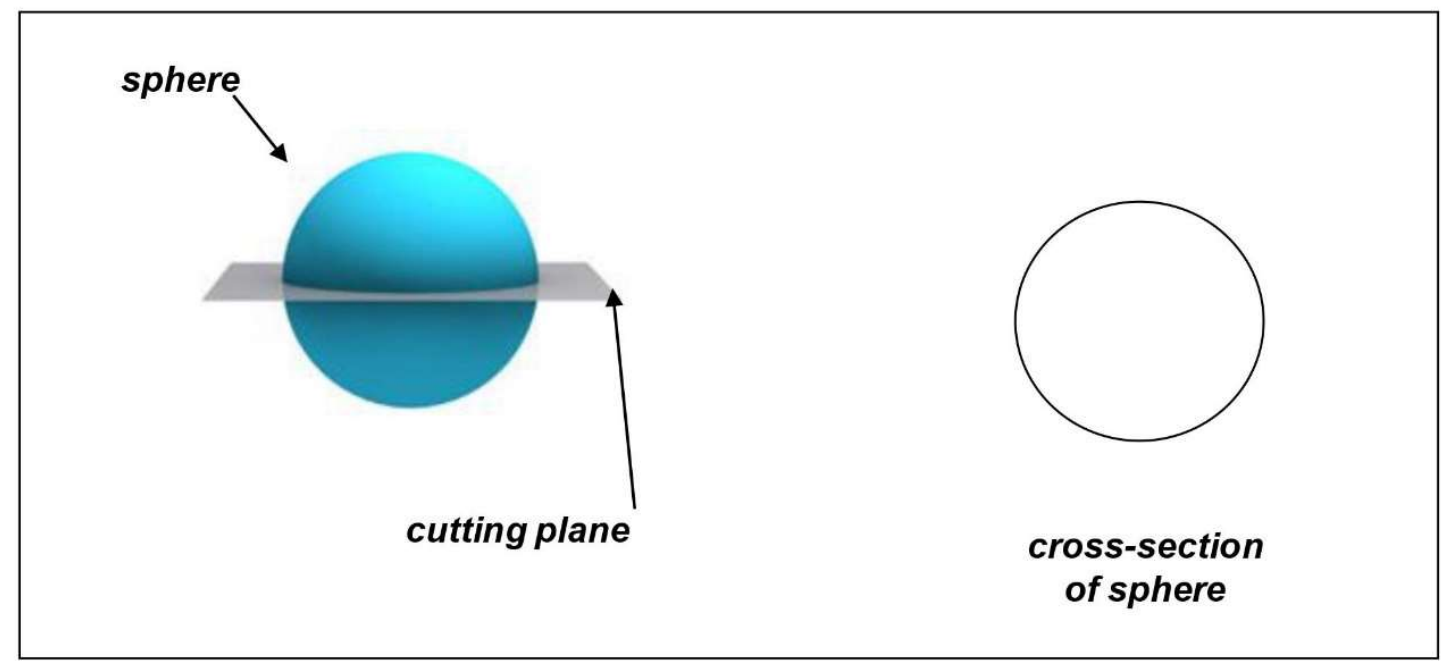


Sample Problem

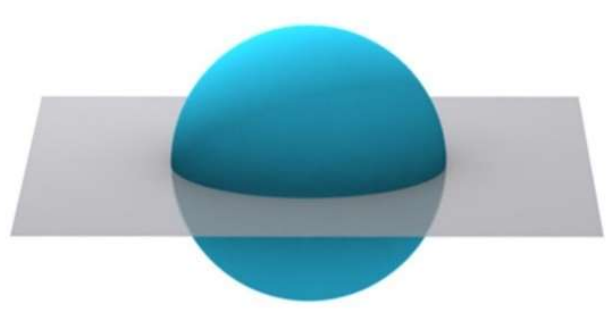

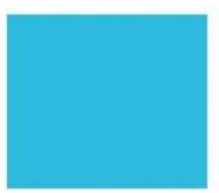

(a)

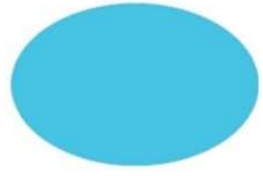

(b)

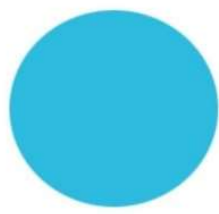

(c)

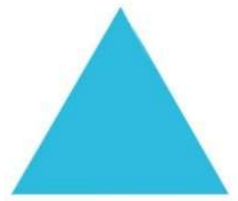

(d)

\section{Instructions:}

Circle the cross-section you would see when the grey cutting plane slices the object. Imagine that you are facing the cutting plane head-on, as if you were looking in a mirror. Make your choice based on the shapes of the possible answers, not their sizes.

This is an untimed test. Work at your own pace.

You can ask the experimenter a question at any time. 
Problem 1

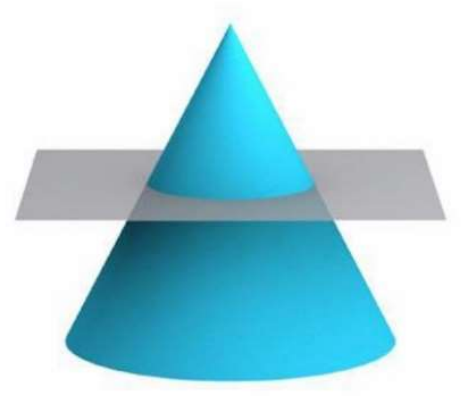

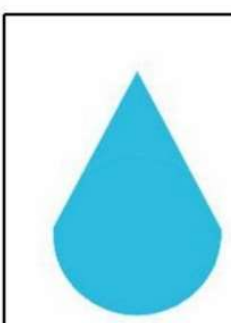

(a)

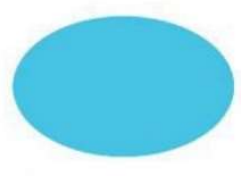

(b)

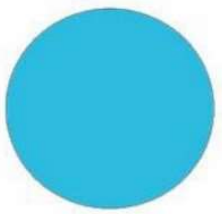

(c)

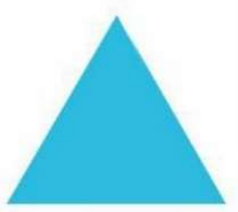

(d)

Problem 2

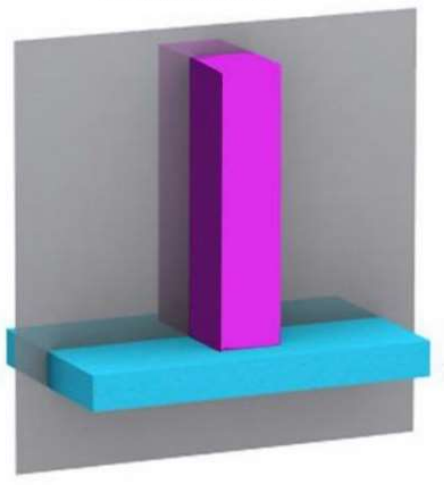

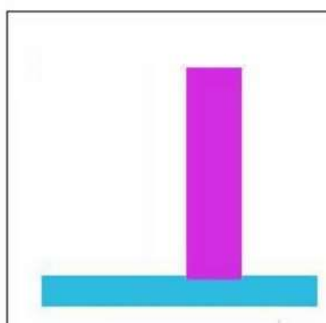

(a)

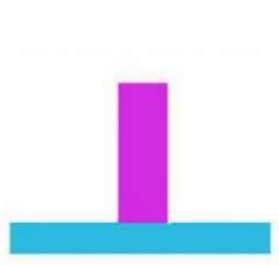

(b)

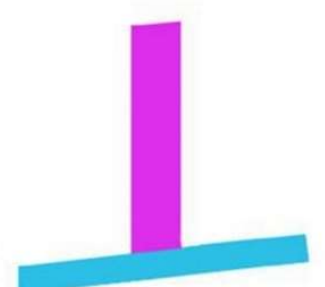

(c)

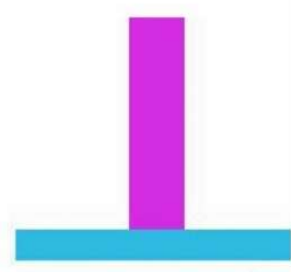

(d) 
Problem 3
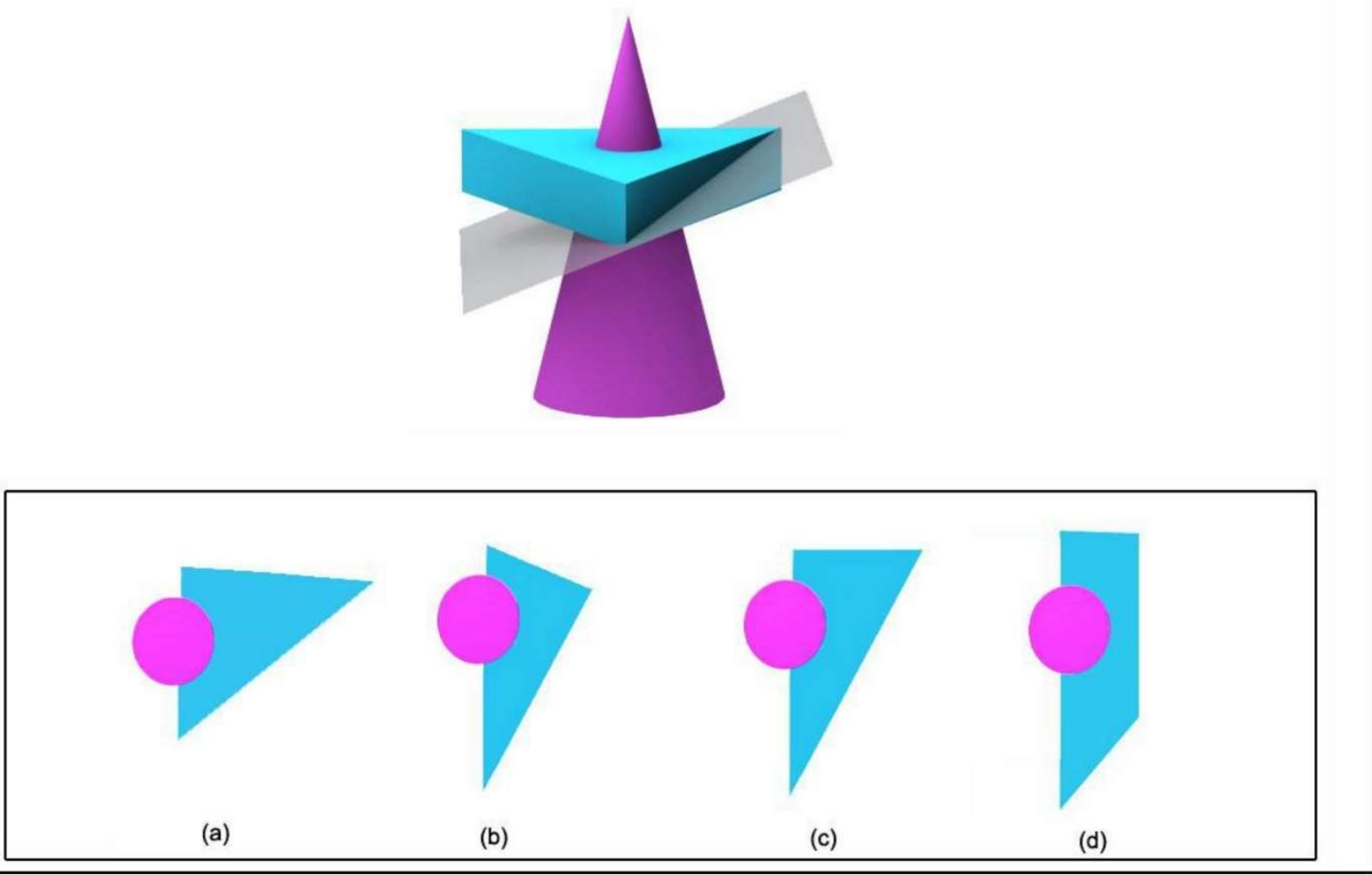

Problem 4
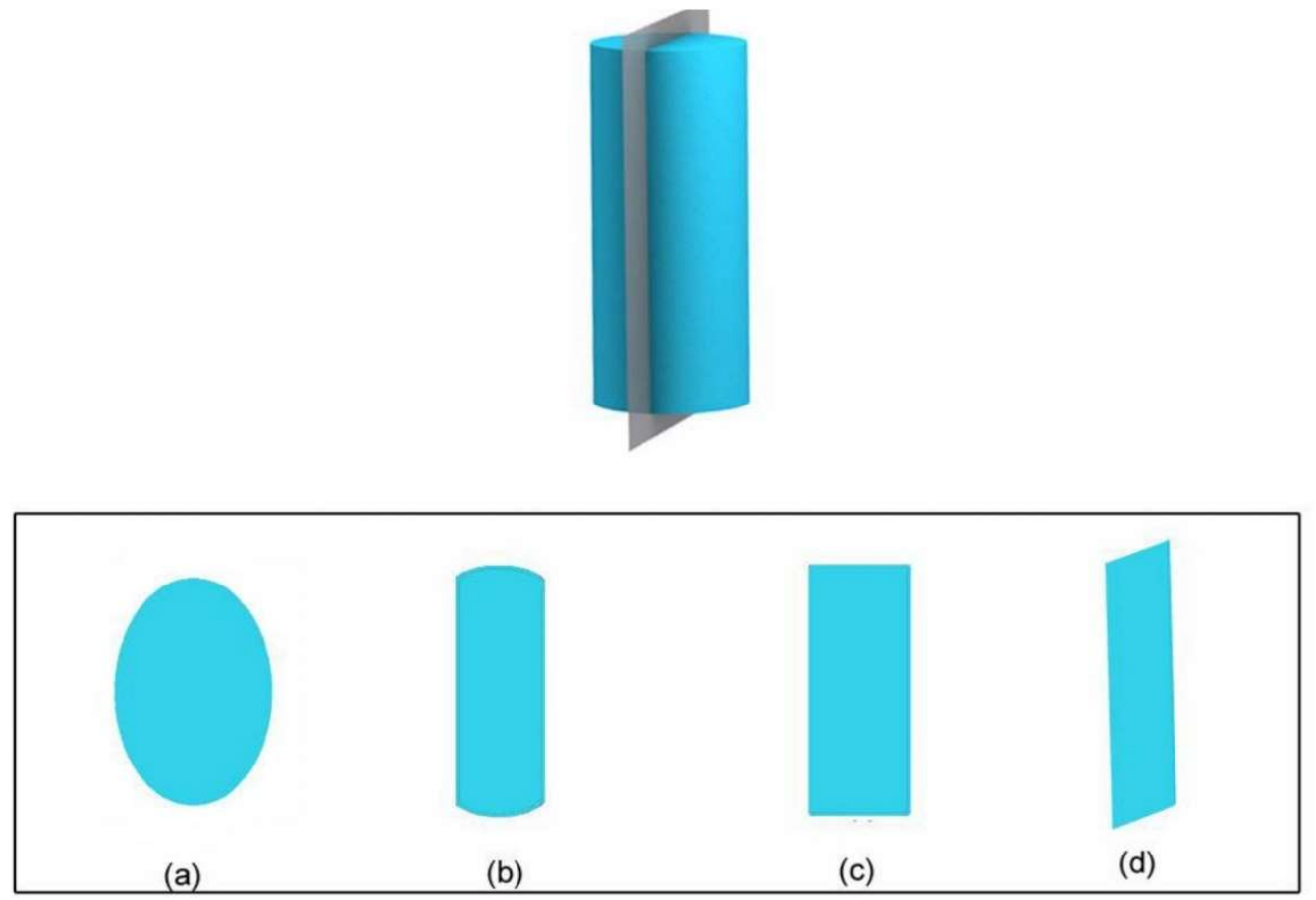


\section{Problem 5}

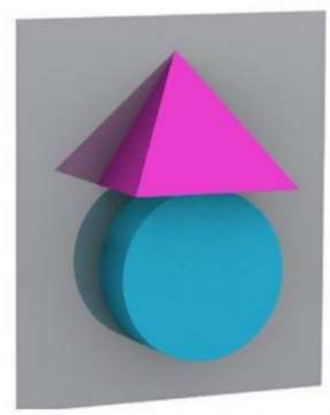

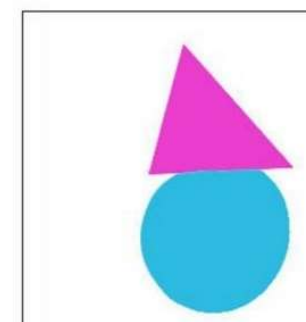

(a)

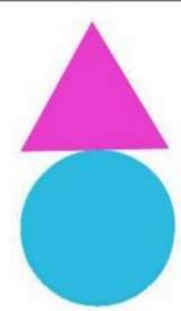

(b)

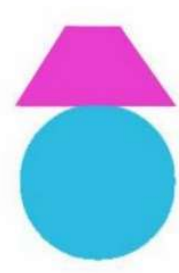

(c)

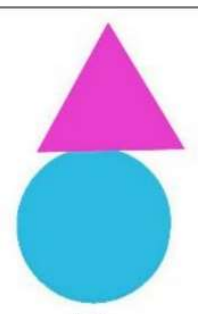

(d)

\section{Problem 6}
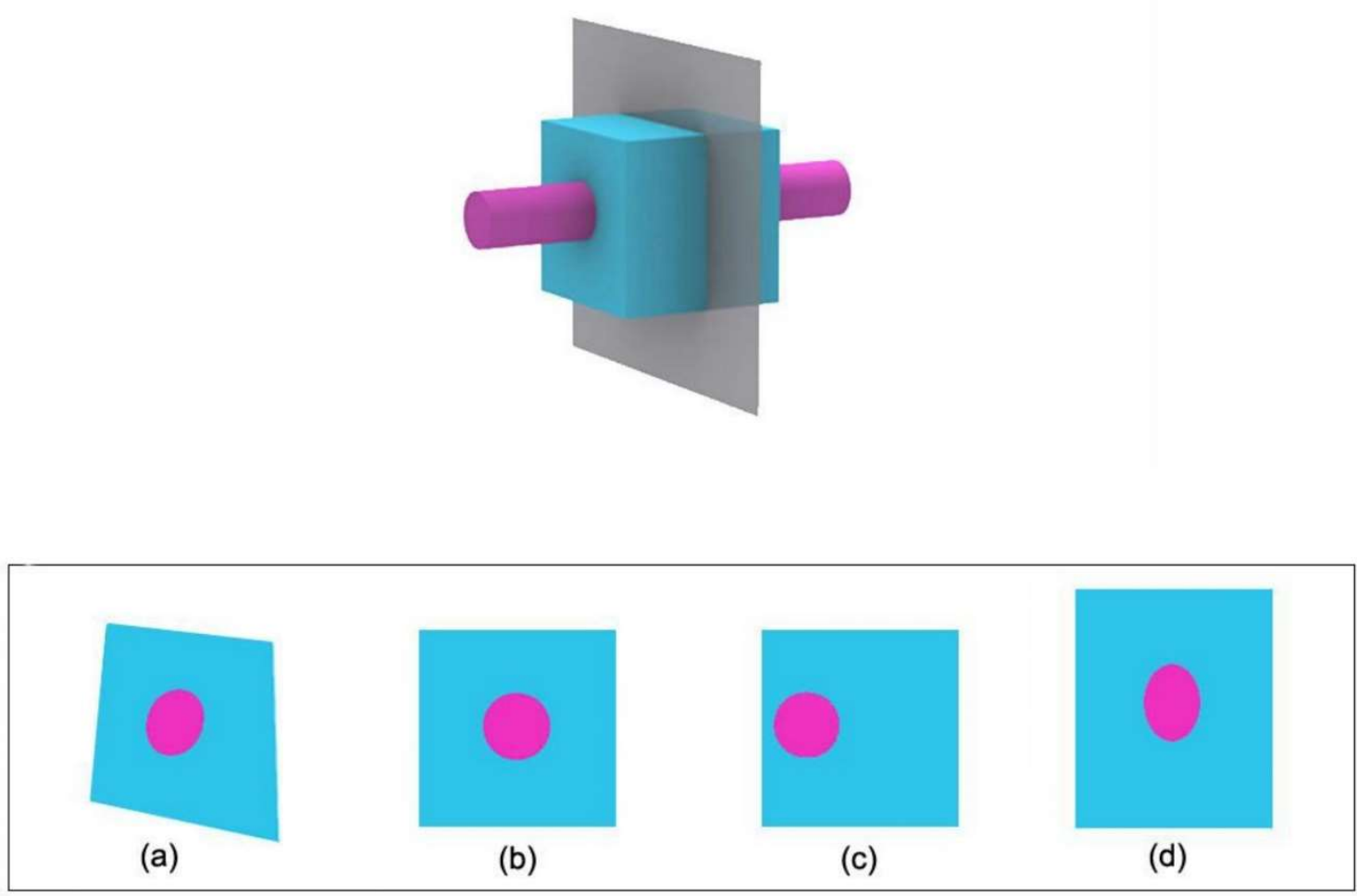


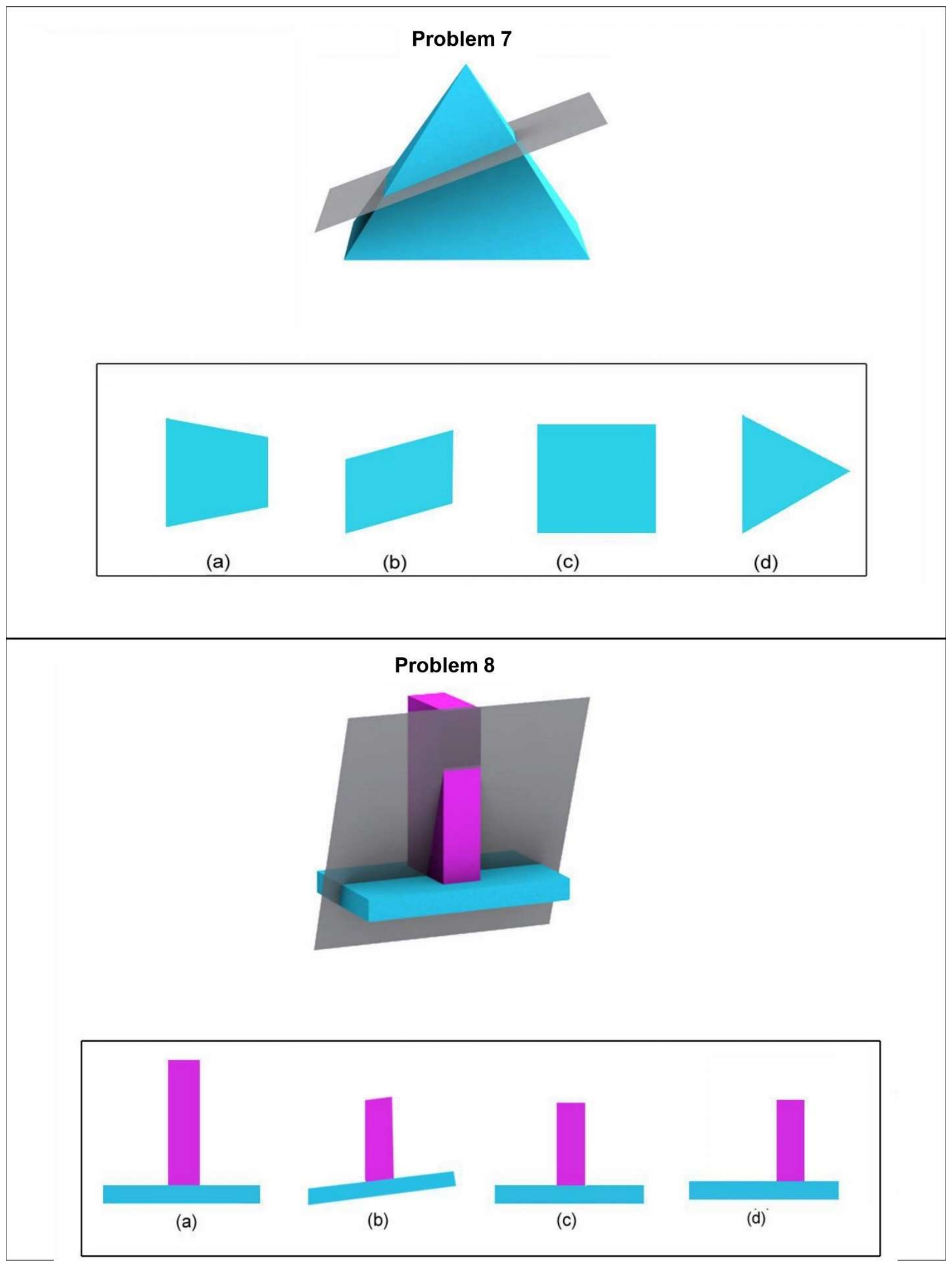




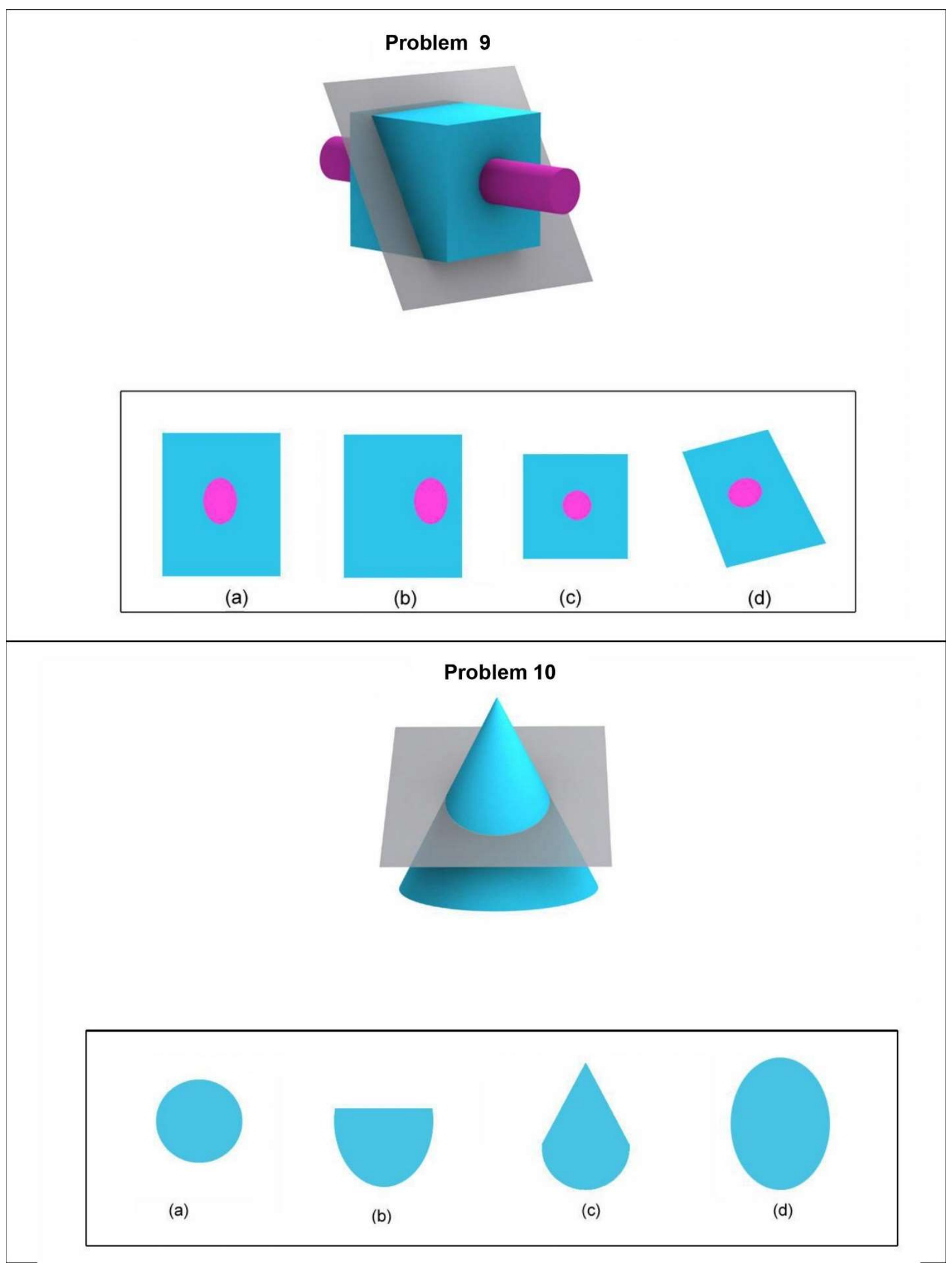




\section{Problem 11}
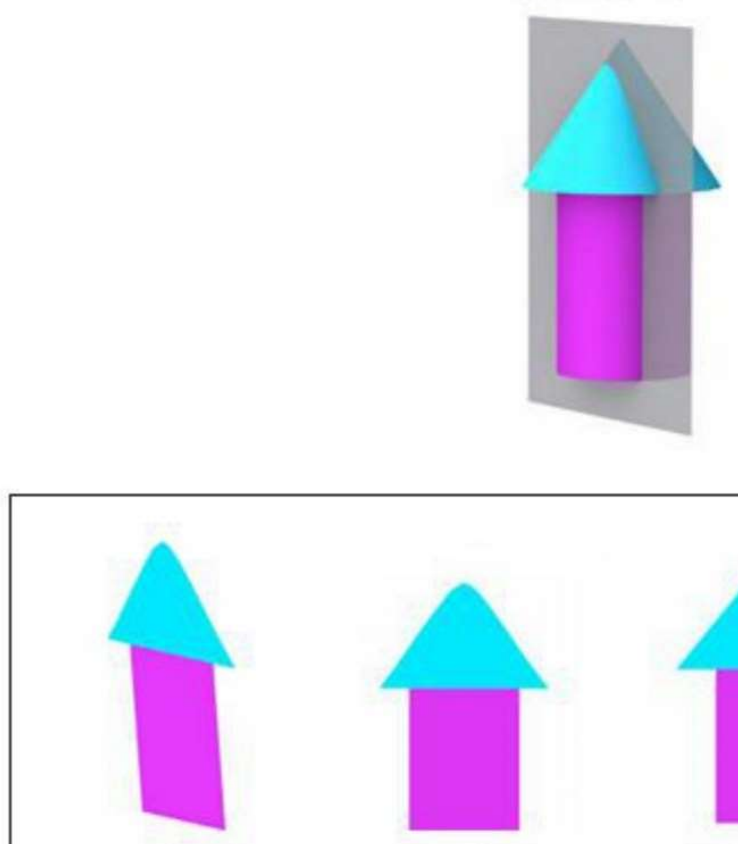

(a)
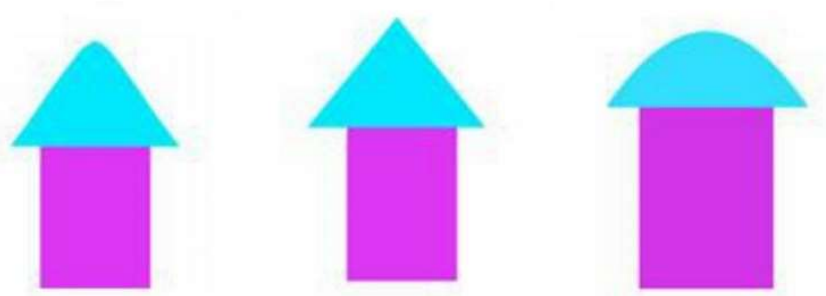

(b)

(c)

(d)

\section{Problem 12}

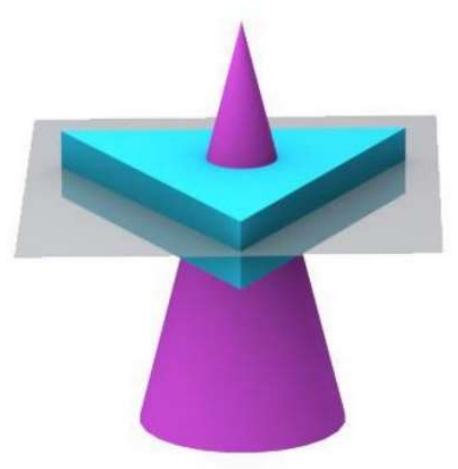

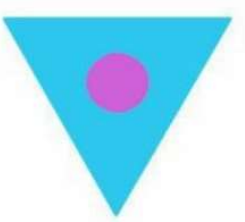

(a)

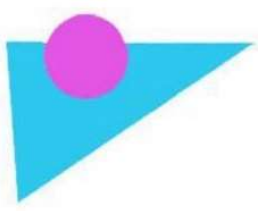

(b)

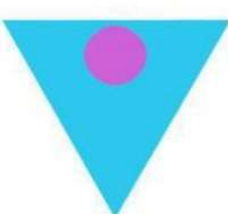

(c)

(d) 

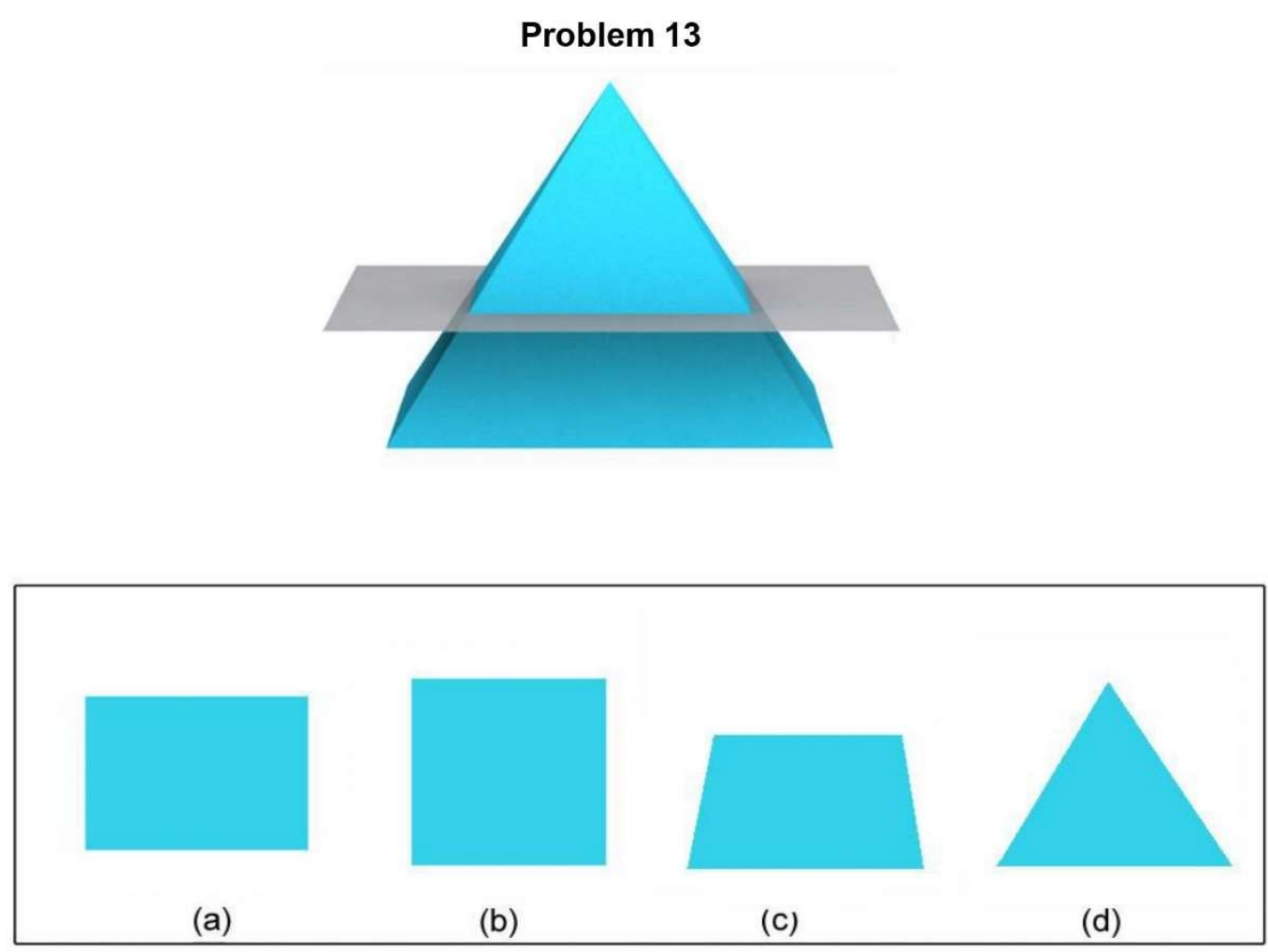

Problem 14

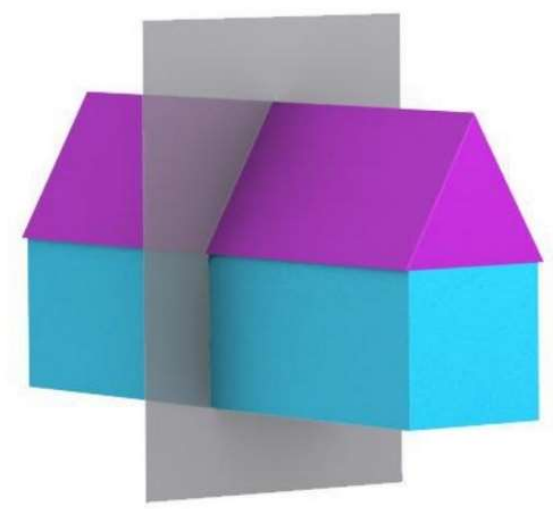

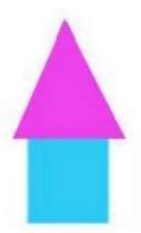

(a)

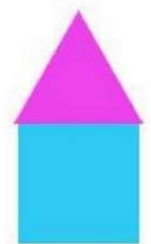

(b)

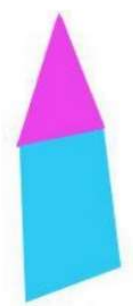

(c)

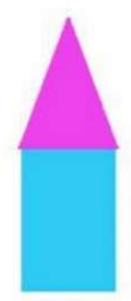

(d) 


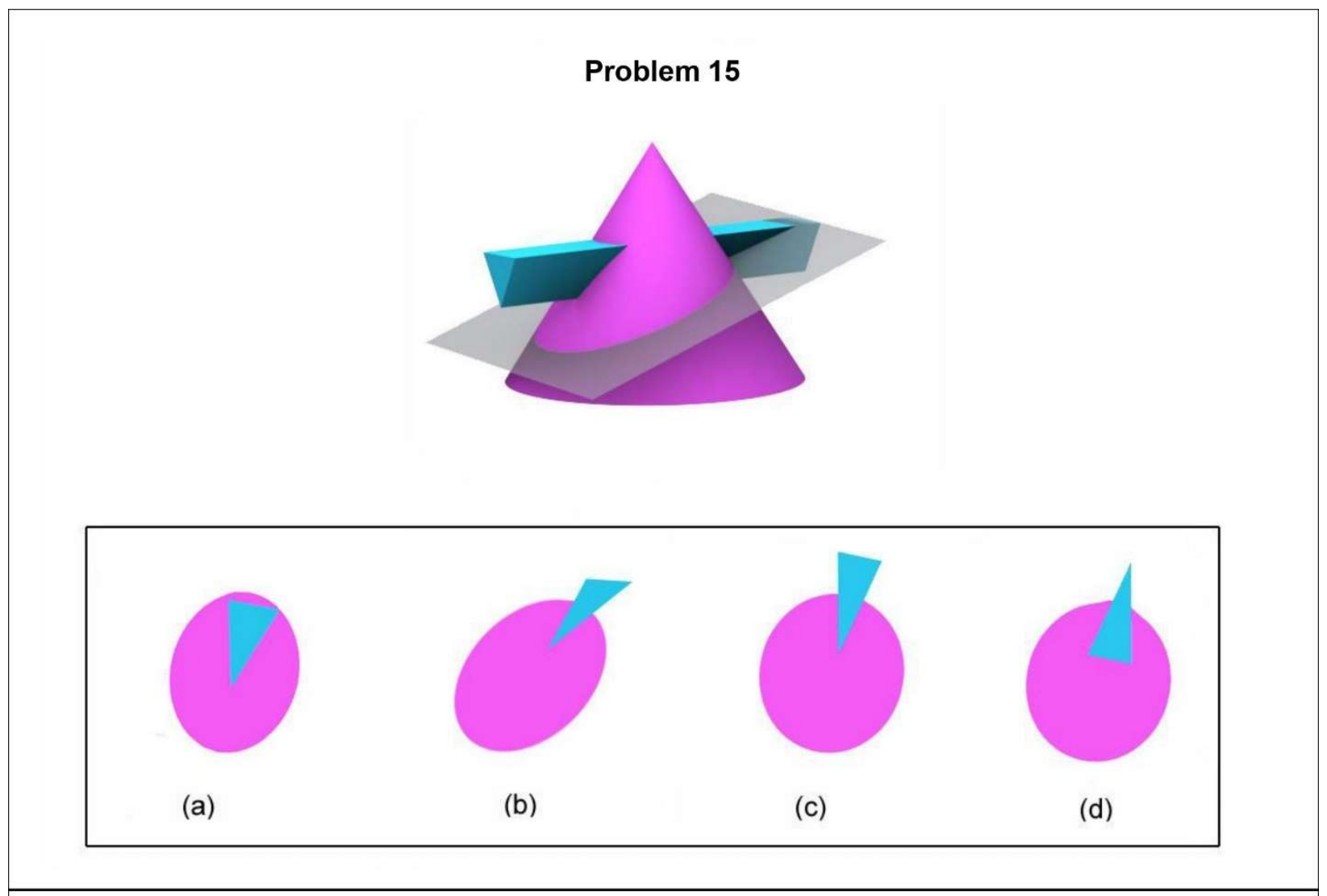

Problem 16

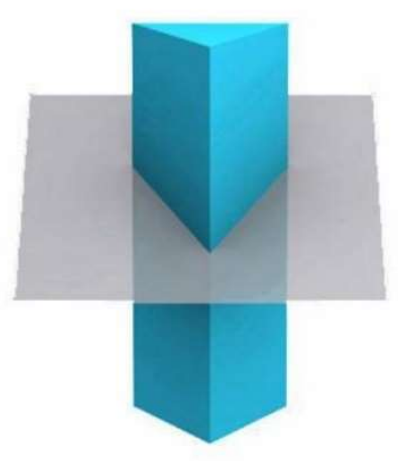

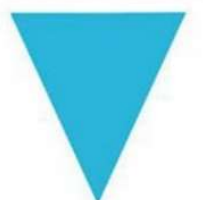

(a)

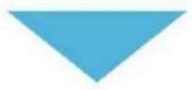

(b)

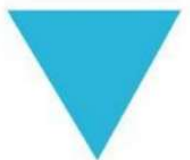

(c)

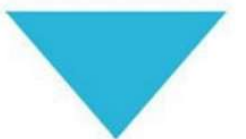

(d) 


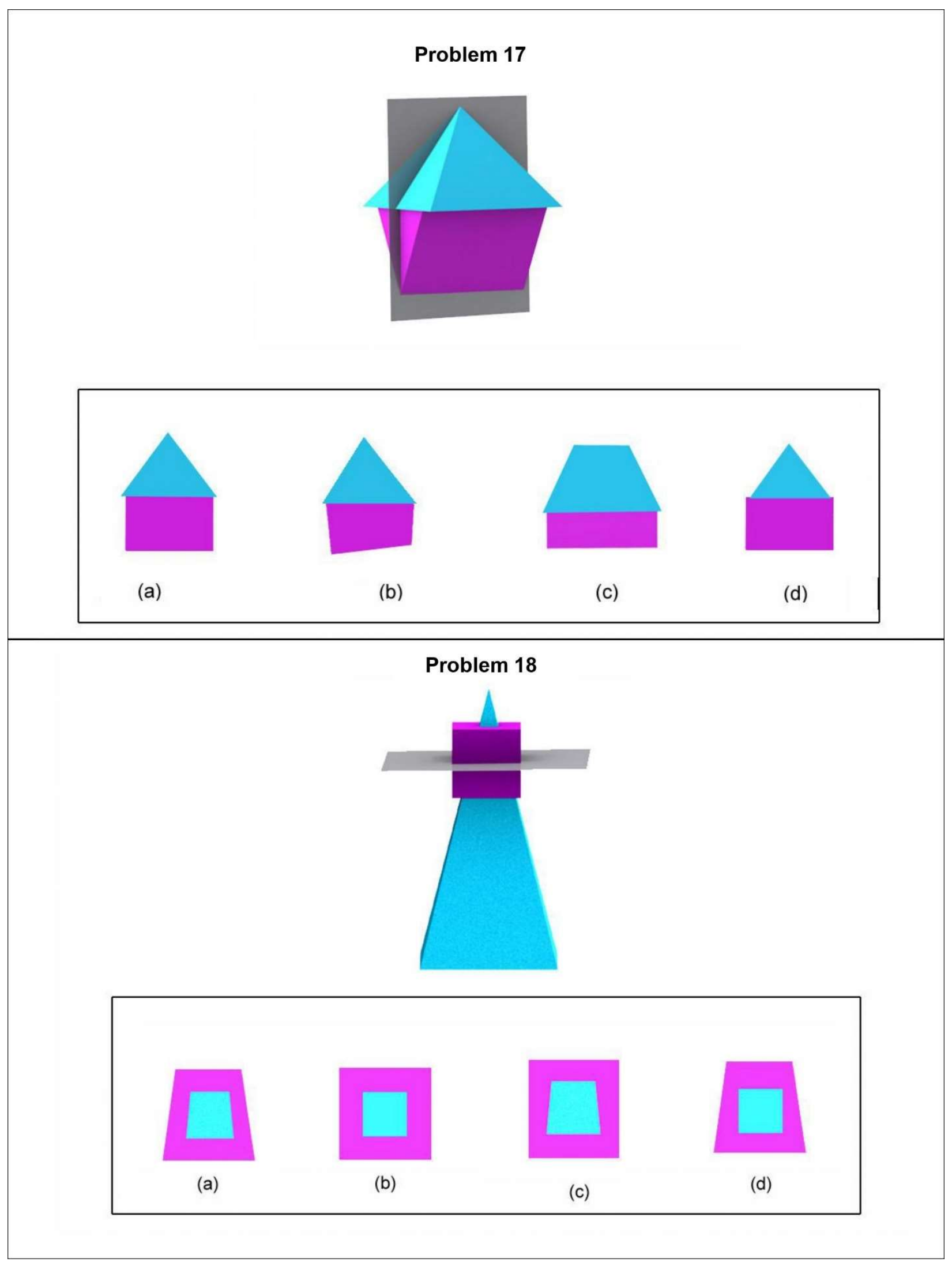




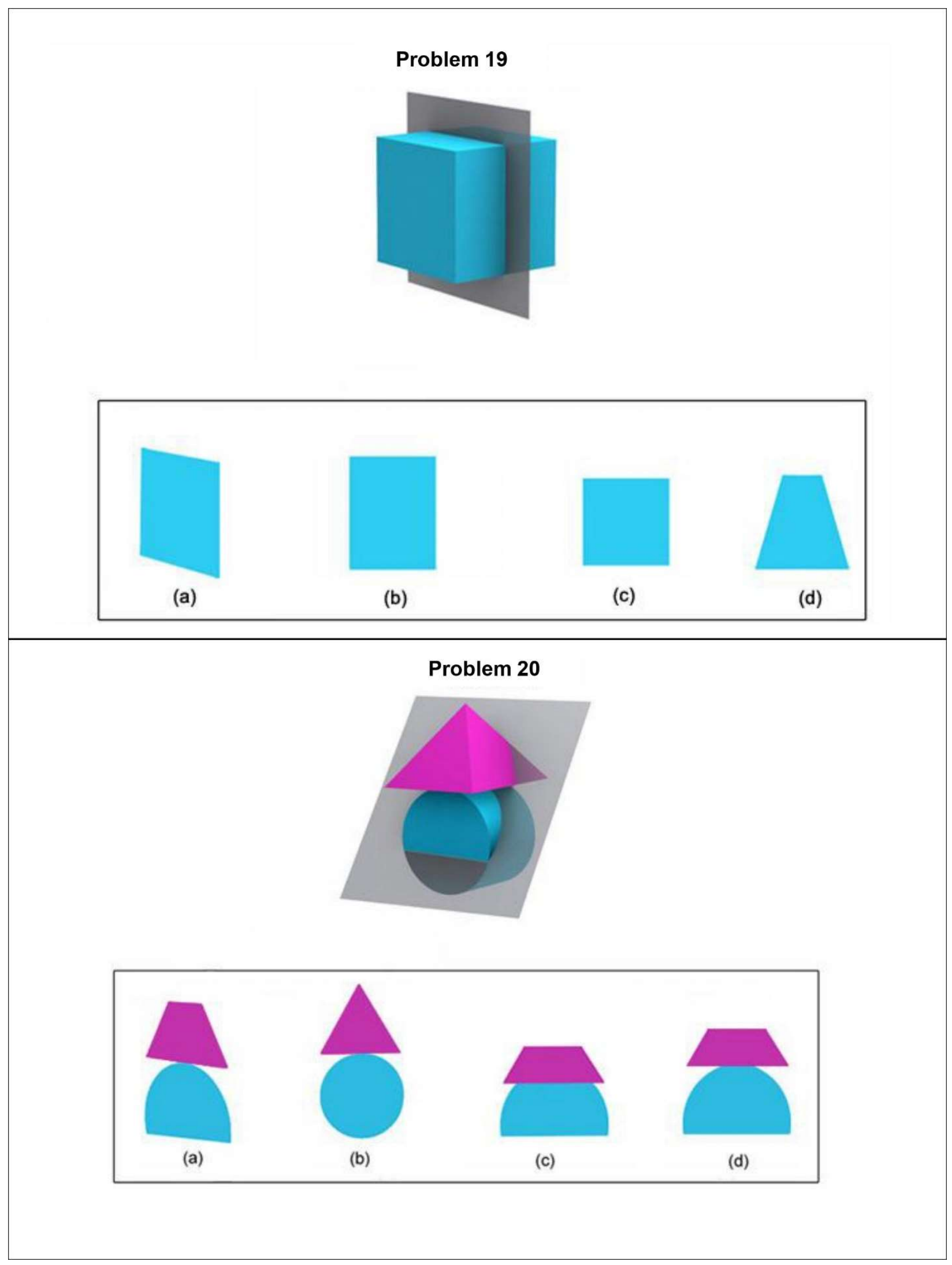




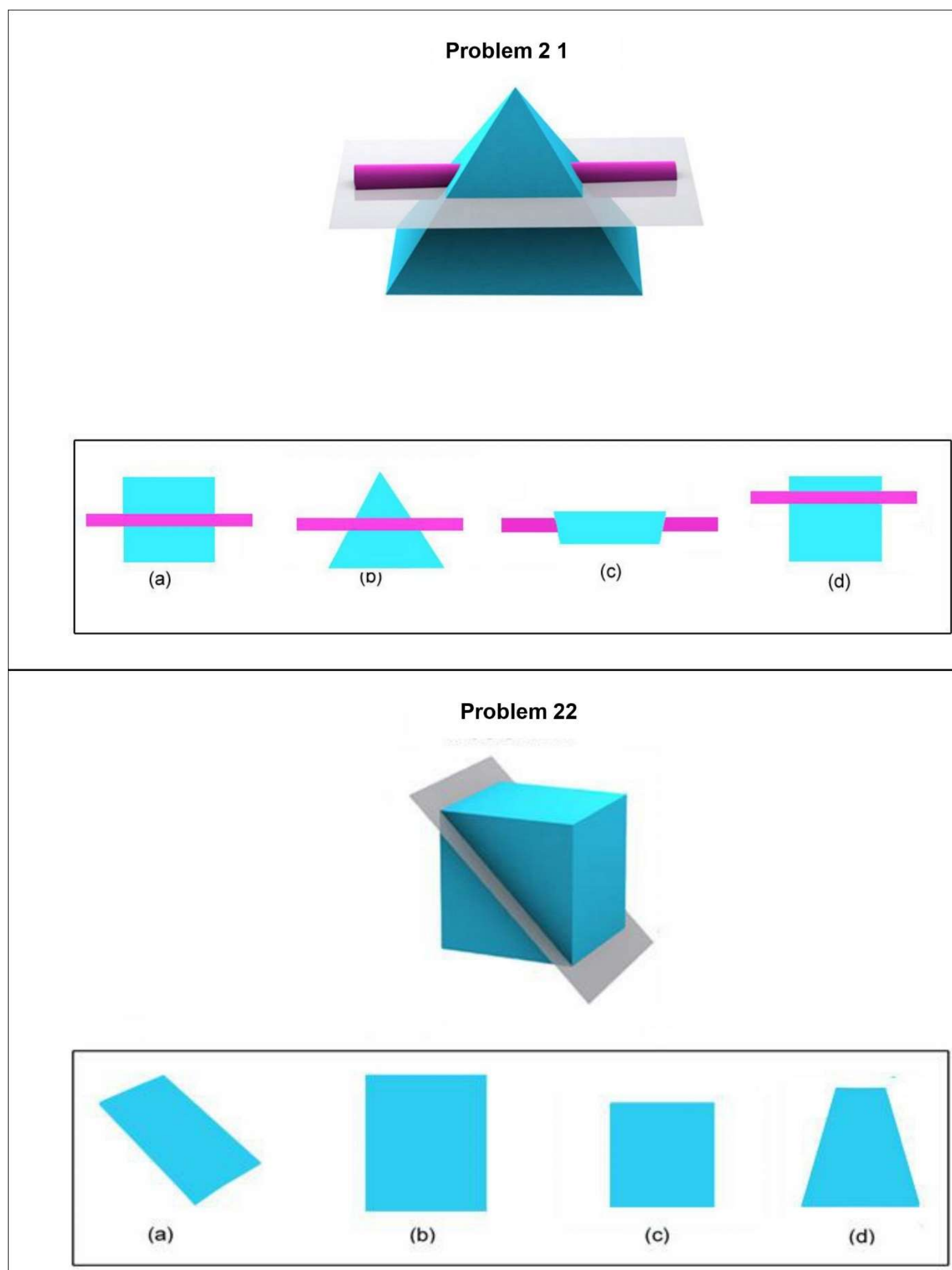




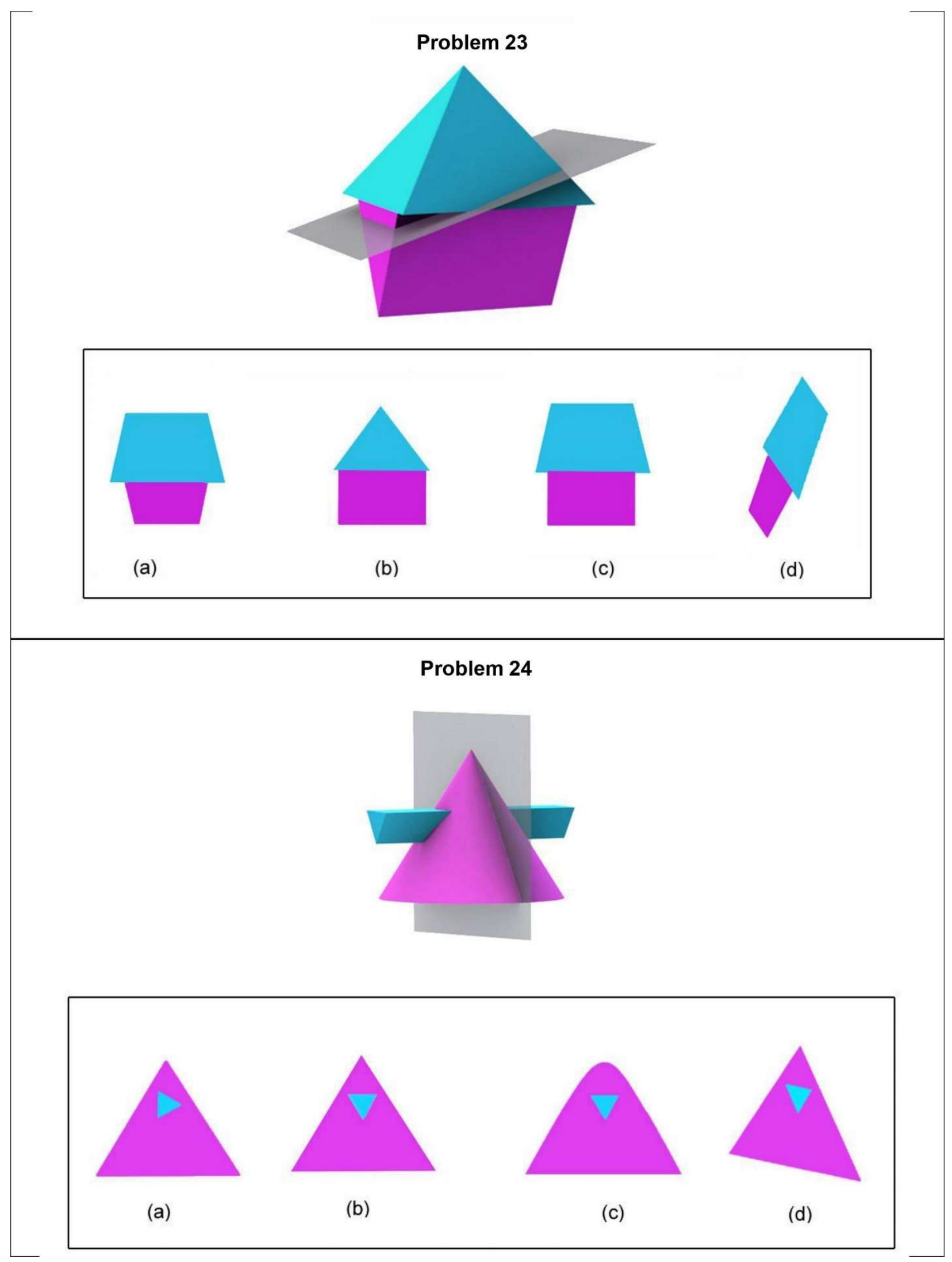




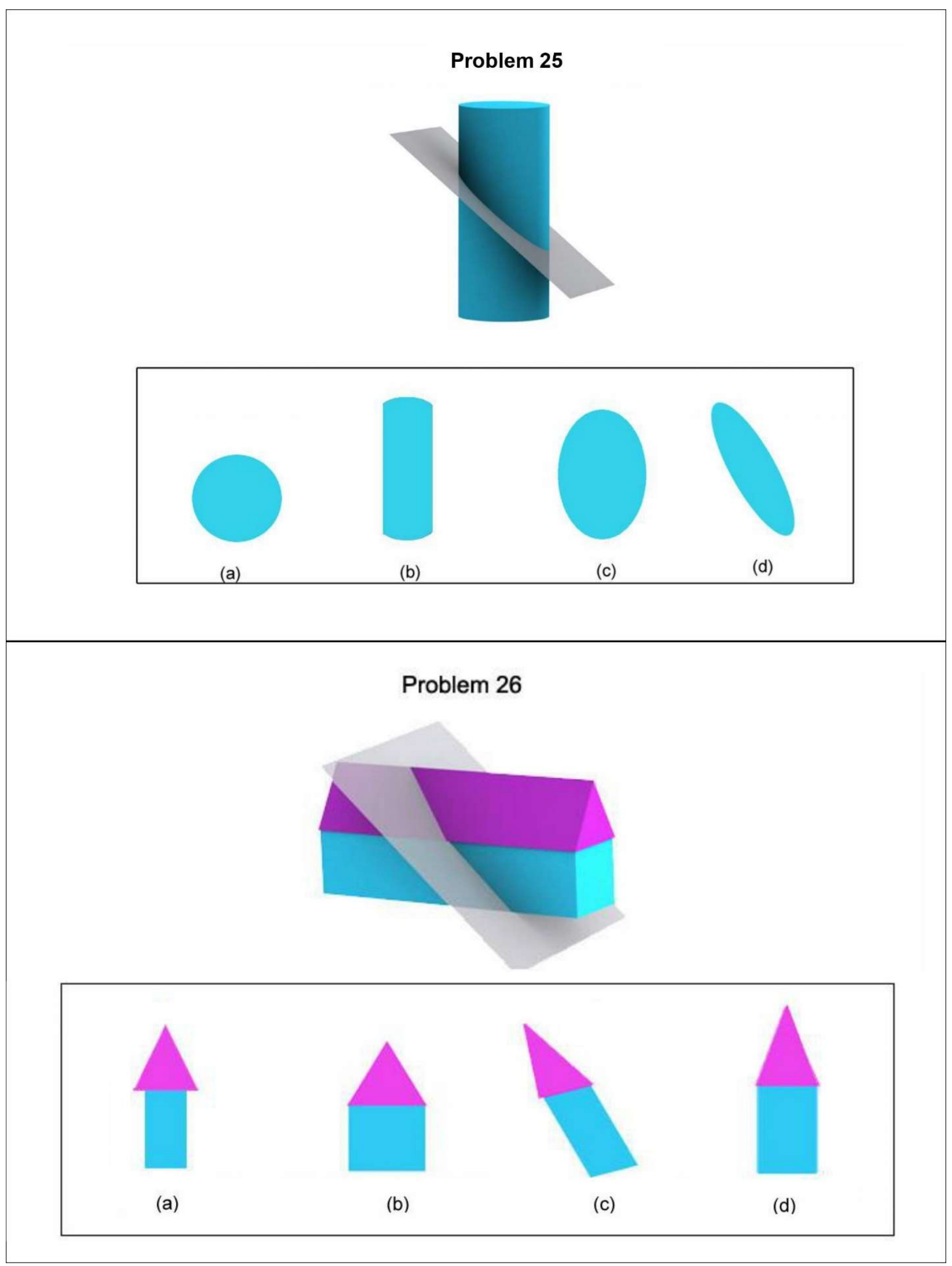




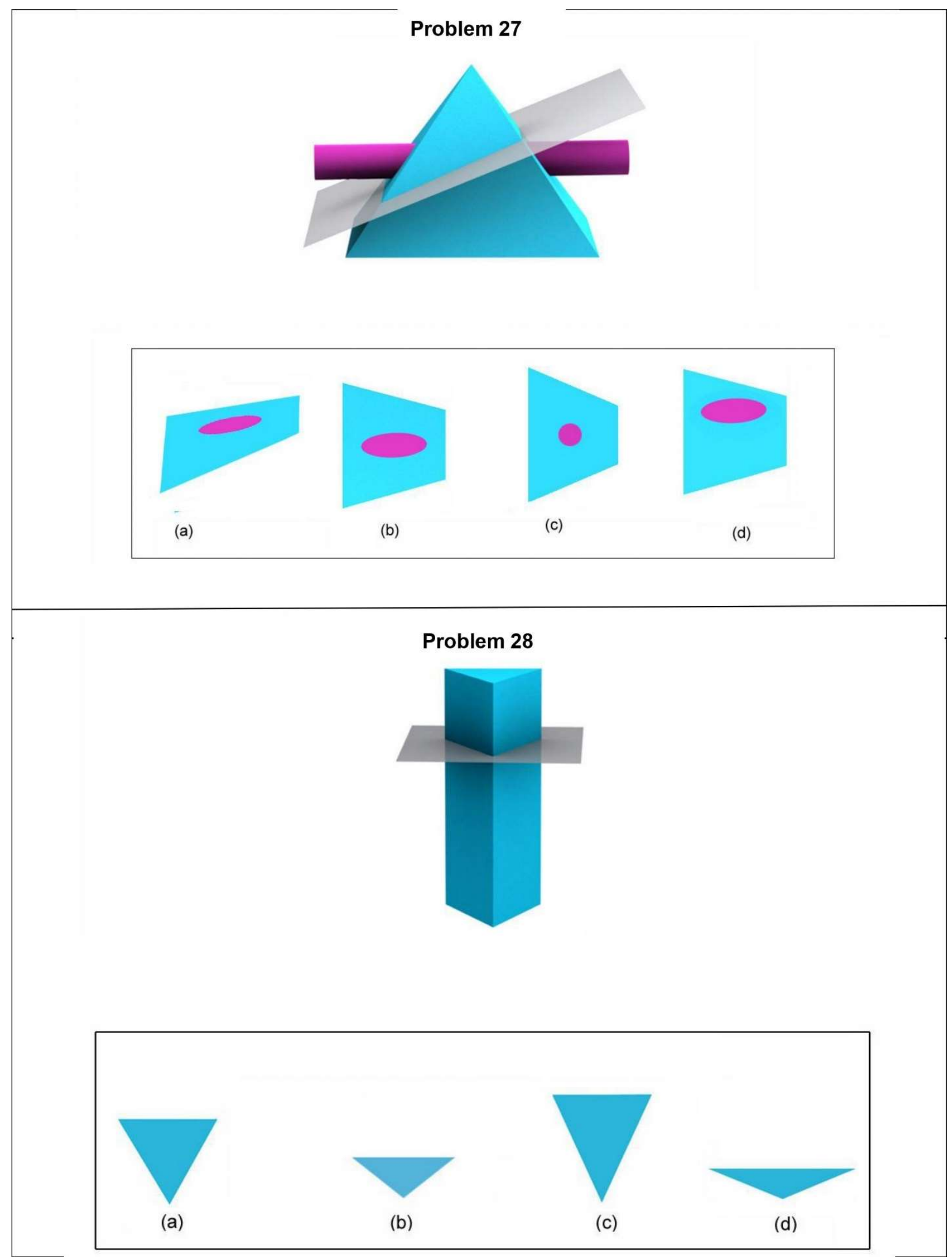




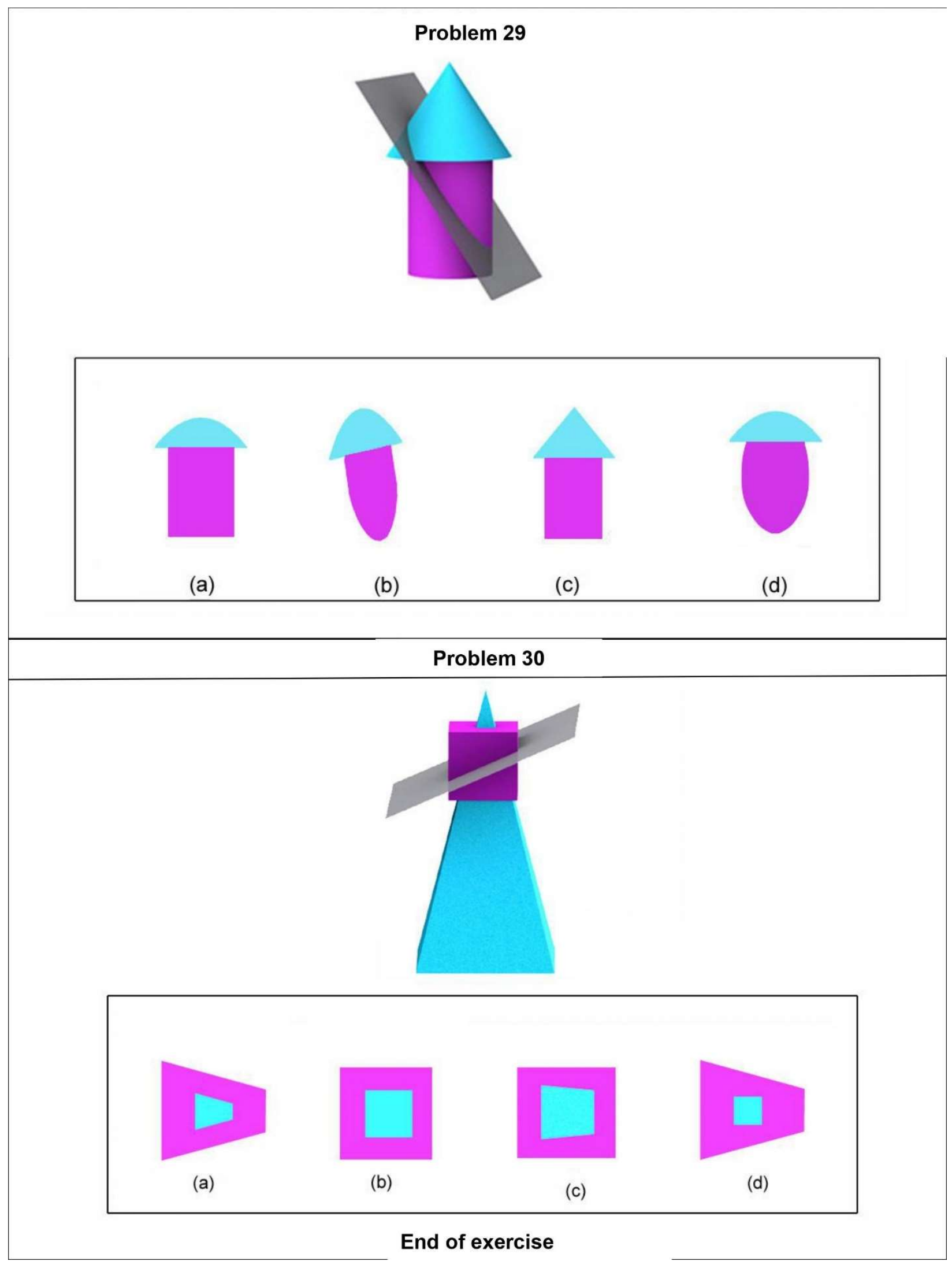


Appendix B: Spatial Reasoning Aptitude Test - Hard

\section{Spatial Reasoning hard}

Practice your spatial reasoning skills in this extremely hard test. Below is a quiz with 12 very difficult spatial reasoning problems. Can you solve them all? Good luck!

Get spatial reasoning tests practice tests used by employers with detailed tips and explanations at JobTestPrep.

\section{Start the test!}

1.

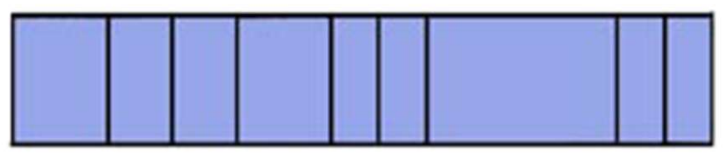

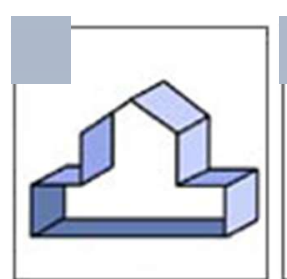

○

2.

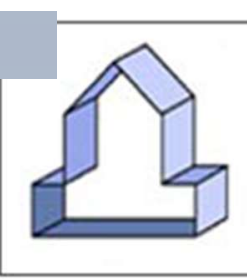

O

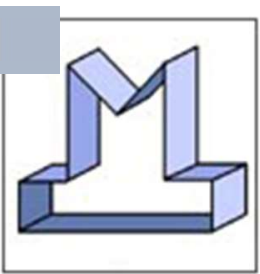

O

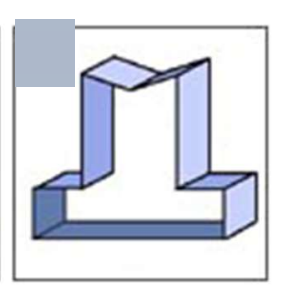

Q

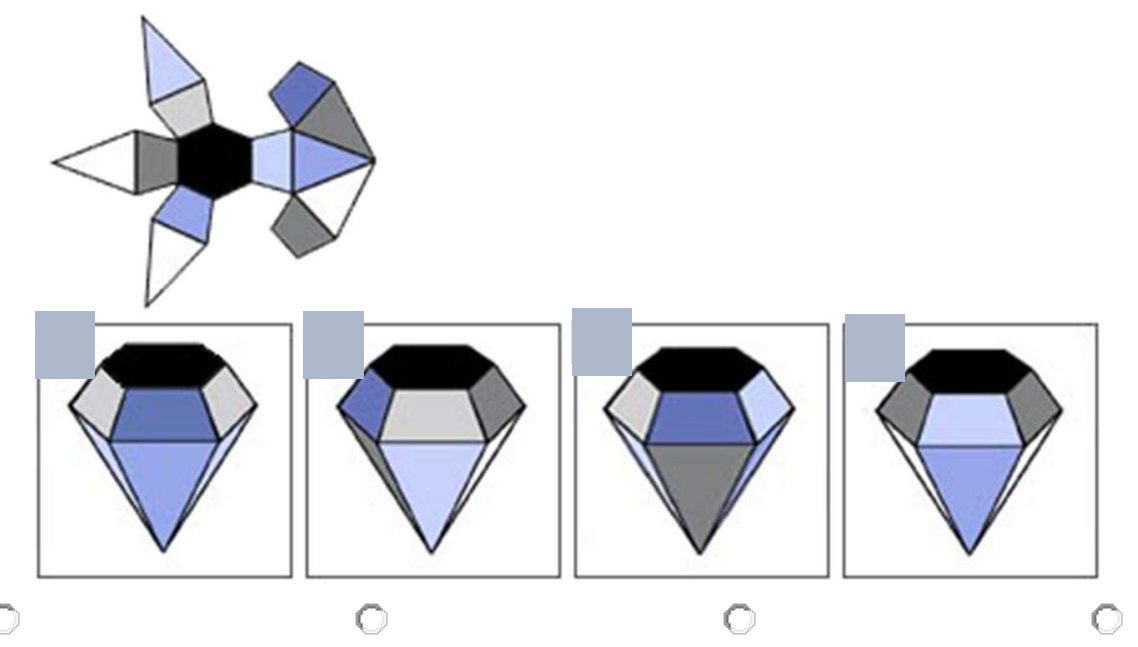


3.

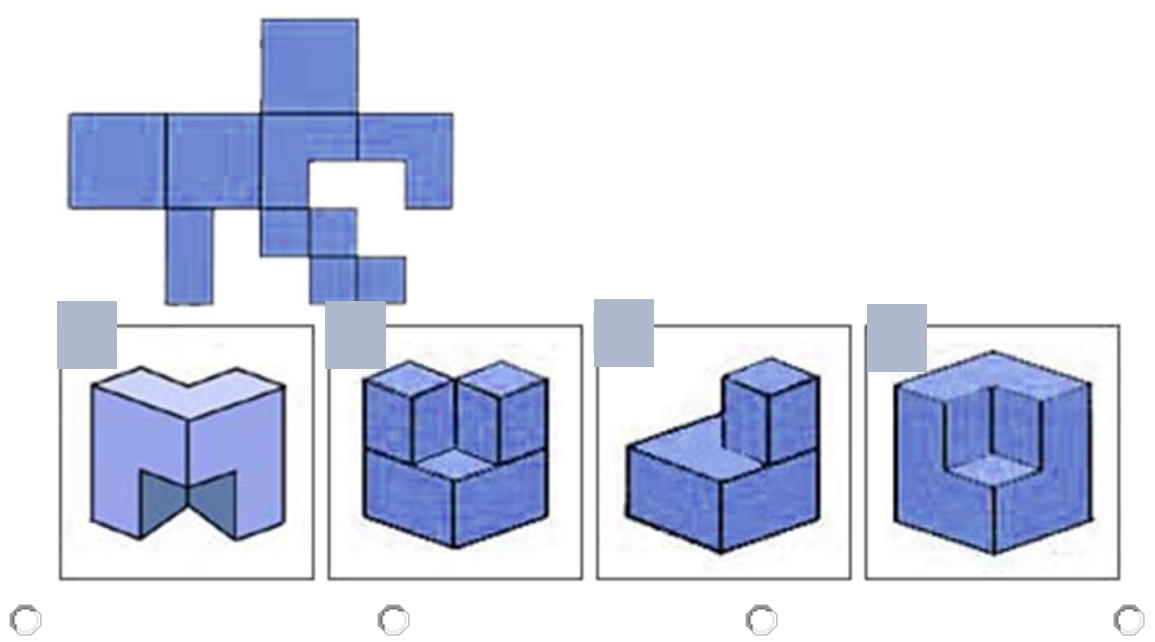

4.

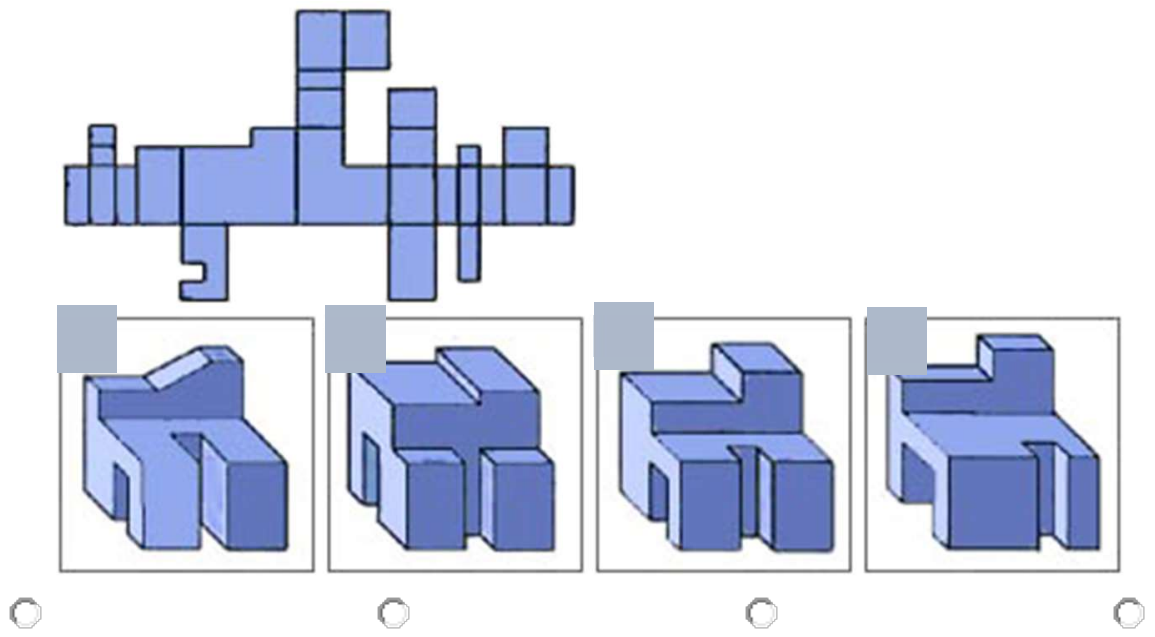

5.
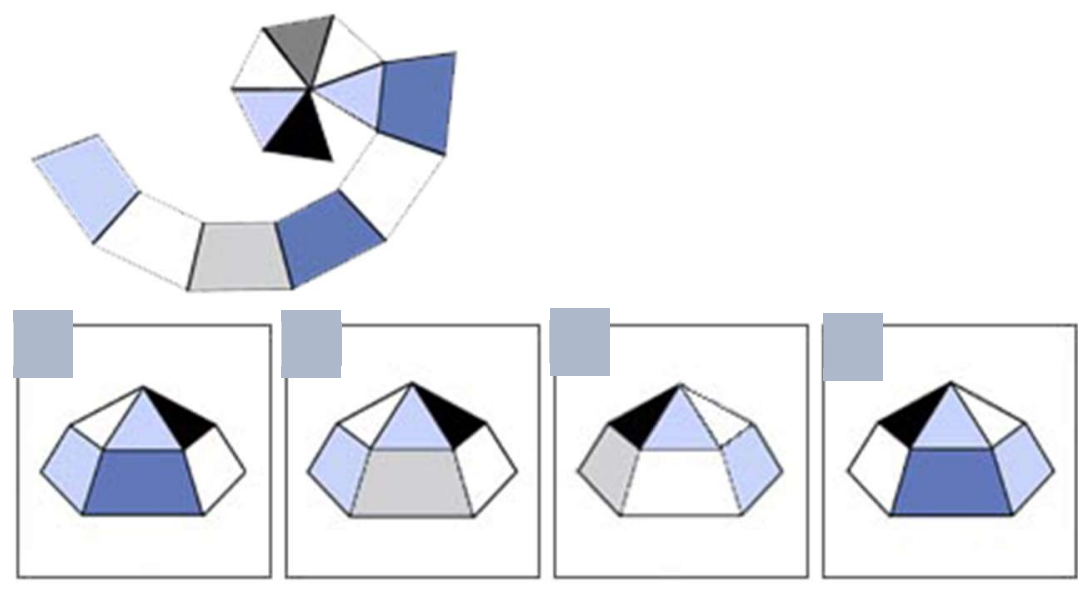

0

0

0

0 
6.

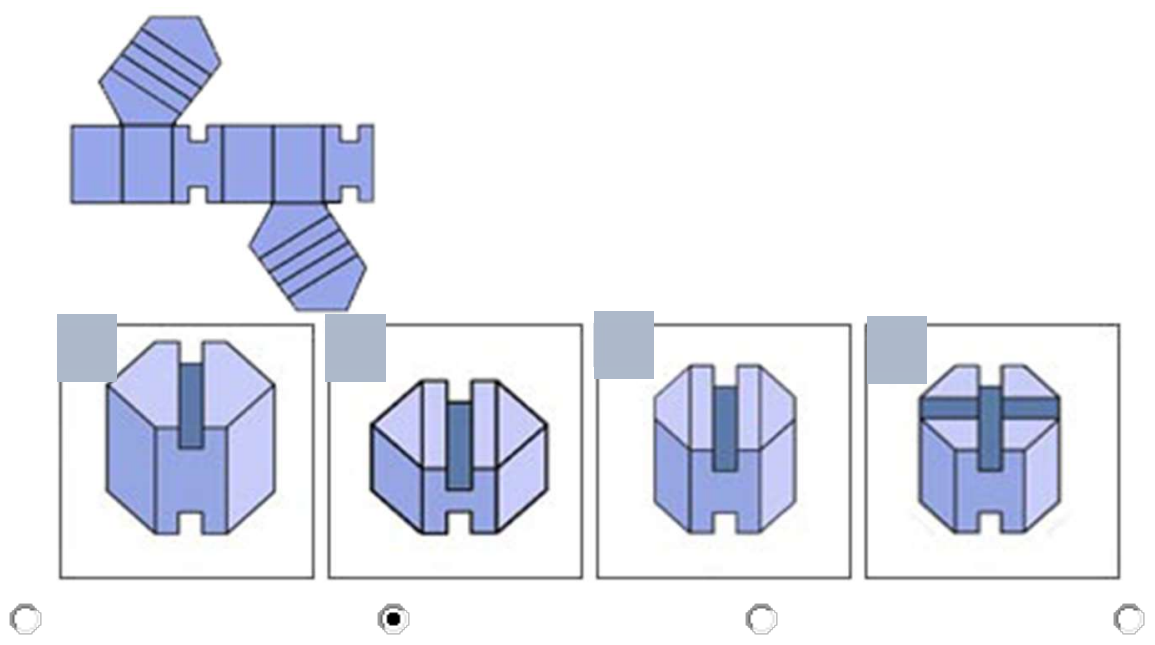

7.

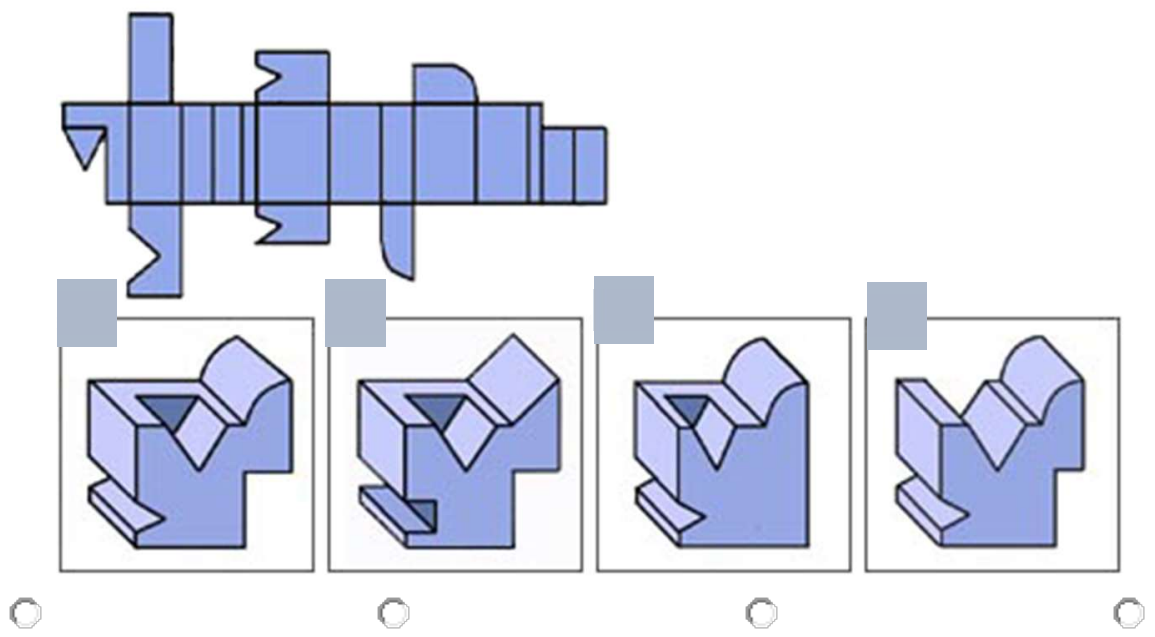

8.

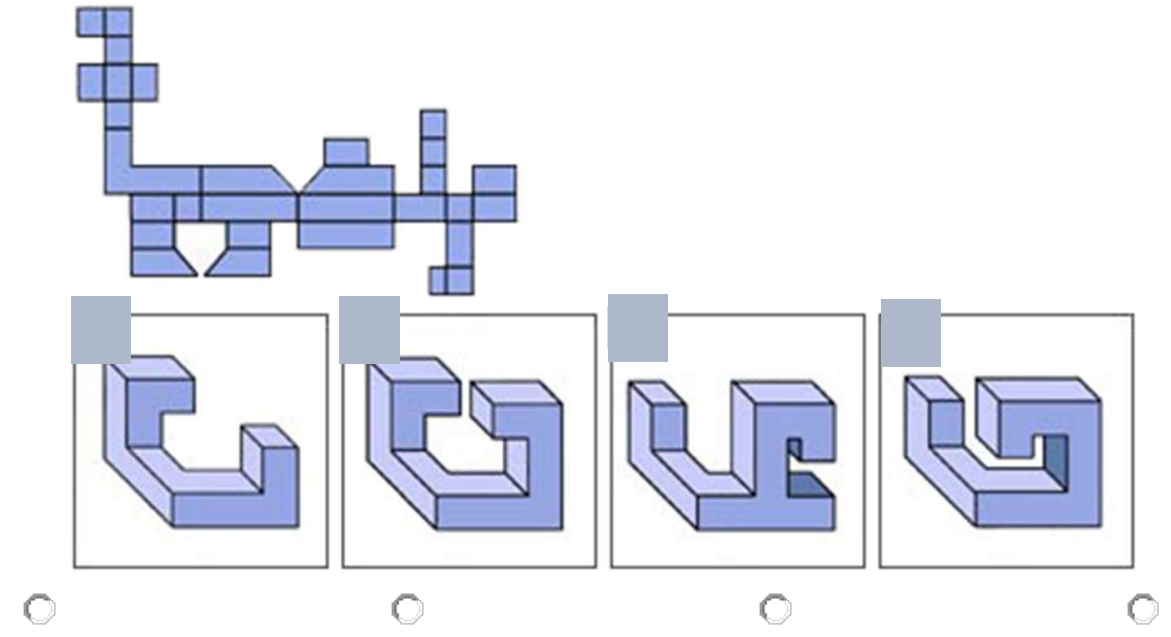


9.

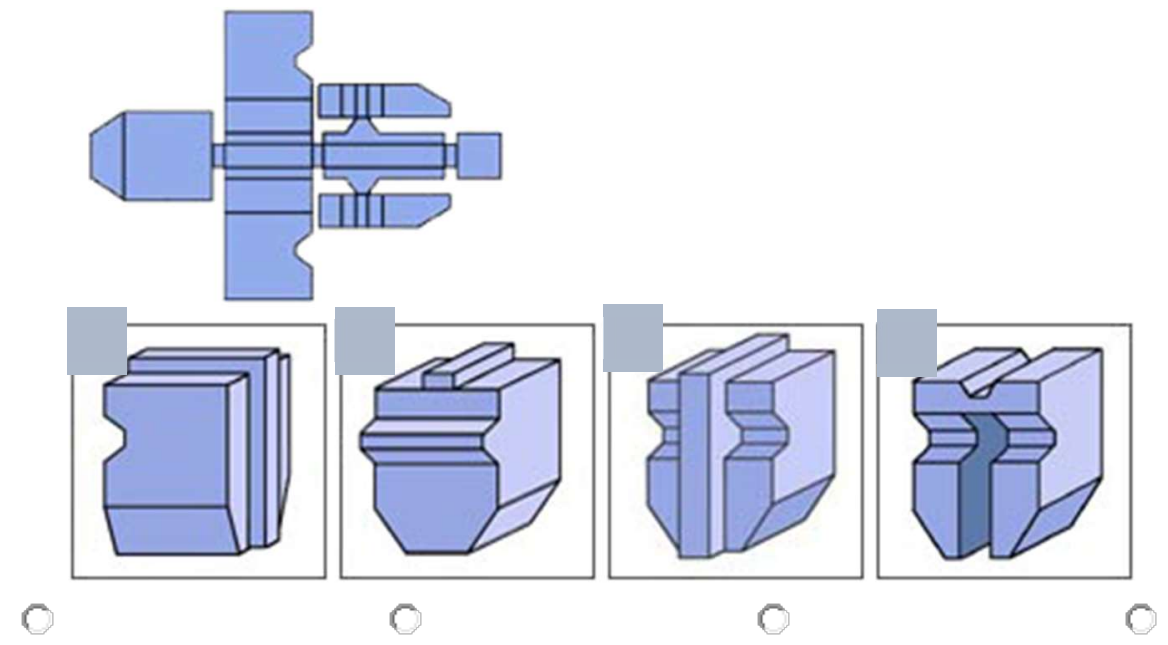

10.

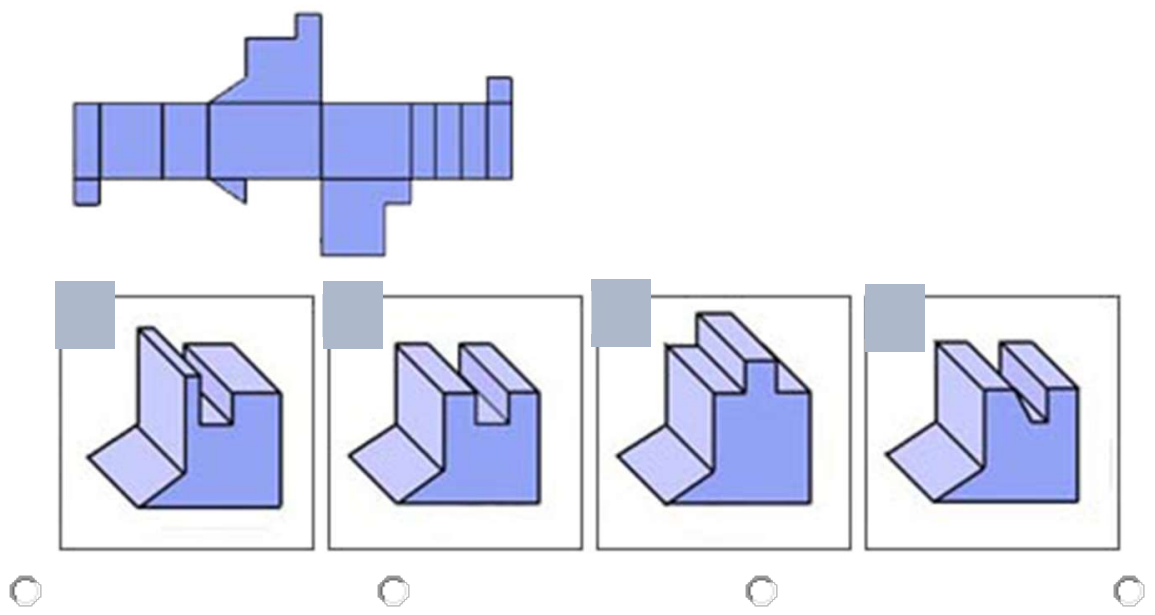

11.
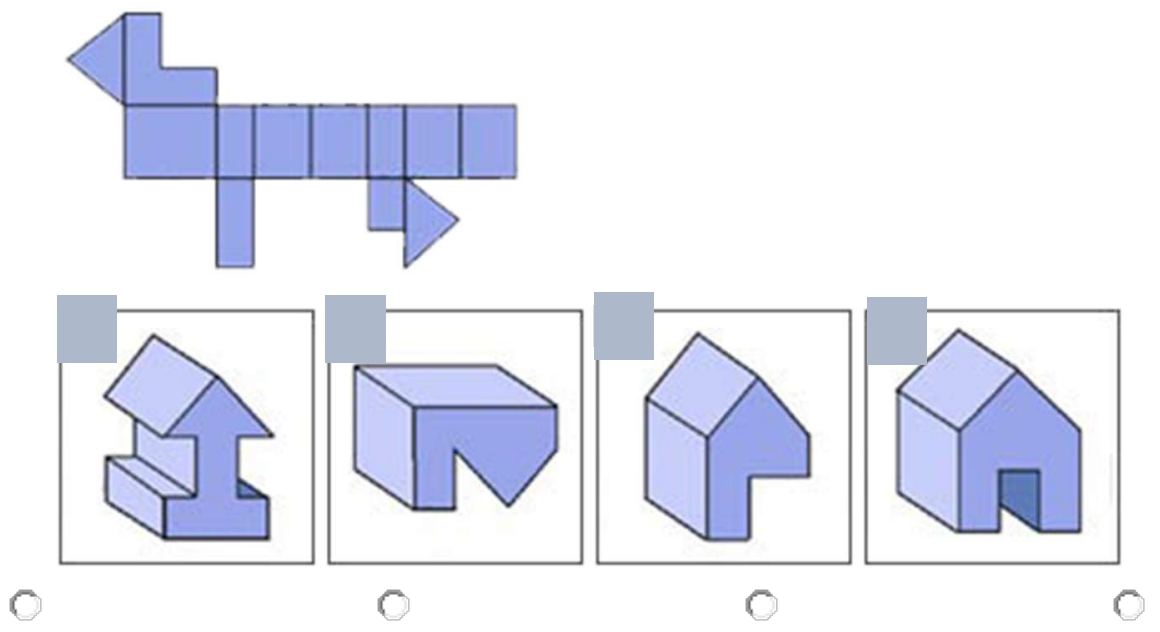
12.

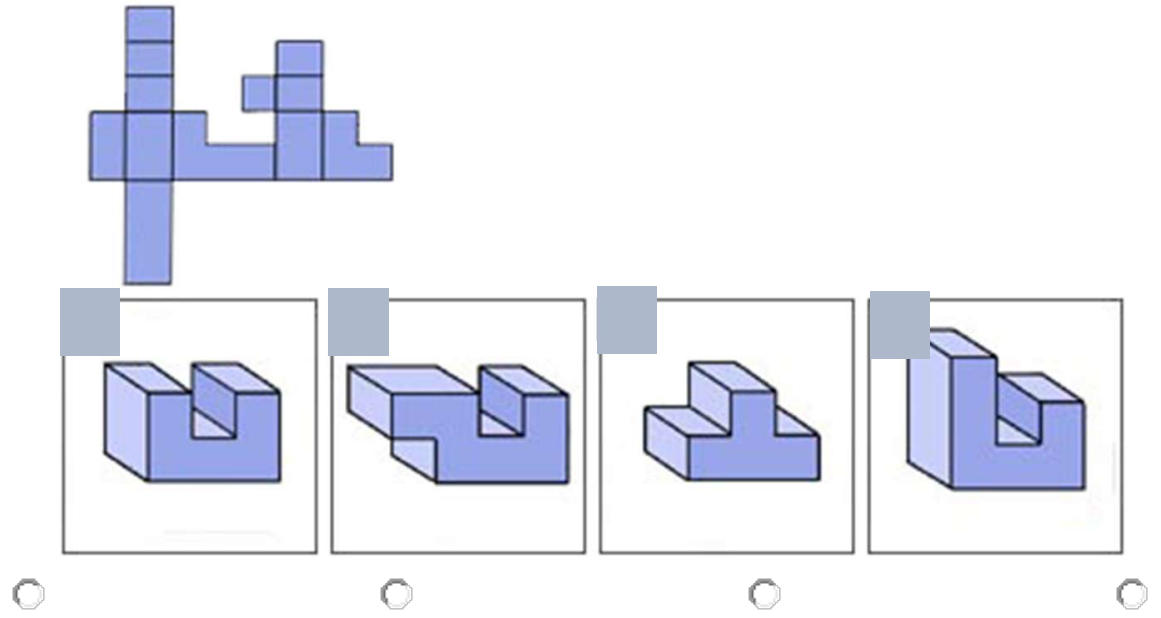


Appendix C: Revised Purdue Spatial Visualization Test: Rotations 


\section{Revised Purdue Spatial Visualization Tests: Visualization of Rotations (Revised PSVT:R)}

So Yoon Yoon

- DO NOT open this booklet until you are instructed to do so.

- DO NOT make any marks in this booklet.

- MARK your answers on the separate answer sheet. 


\section{DIRECTIONS}

This test consists of 30 questions designed to see how well you can visualize the rotation of threedimensional objects. Shown below is an example of the type of question included in the second section.

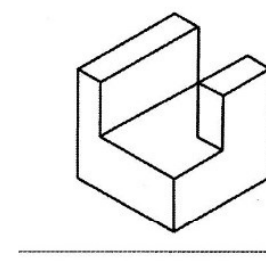

IS ROTATED TO

As

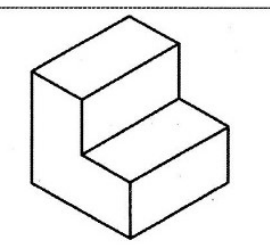

\section{IS ROTATED To}

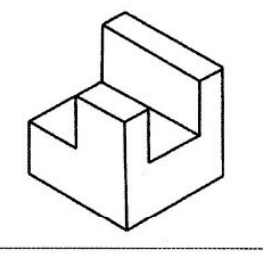

A

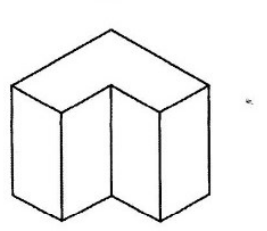

B

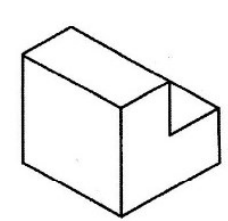

$\mathrm{C}$

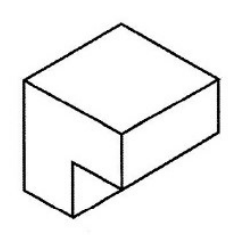

D

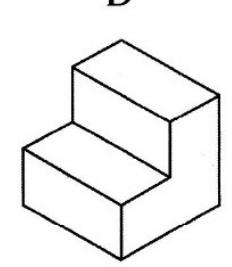

E

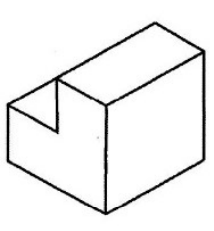

You are to:

1. study how the object in the top line of the question is rotated;

2. picture in your mind what the object shown in the middle line or the question looks like when rotated in exactly the same manner;

3. select from among the five drawings (A, B, C, D, or E) given in the bottom line of the question the one that looks like the object rotated in the correct position.

What is the correct answer to the example shown above? 
Answers A, B, C, and E are wrong. Only drawing D looks like the object rotated according to the given rotation. Remember that each question has only one correct answer.

Now look at the next example shown below and try to select the drawing that looks like the object in the correct position when the given rotation is applied.

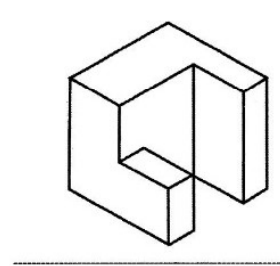

\section{Is ROTATED TO}

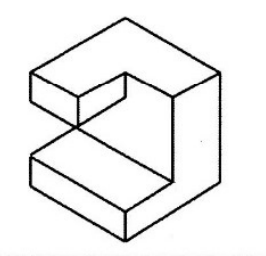

As

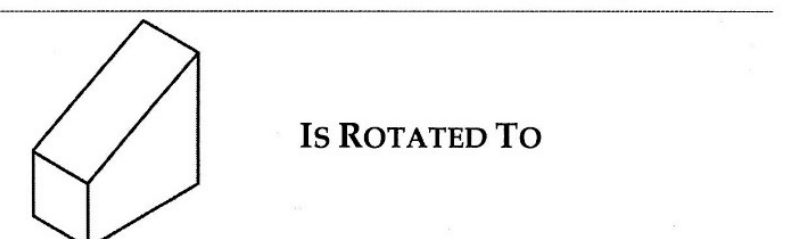

A

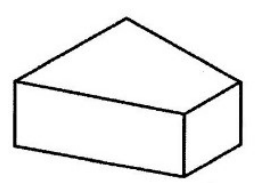

B

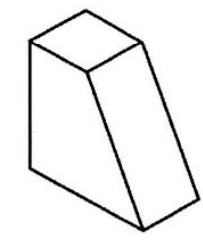

C

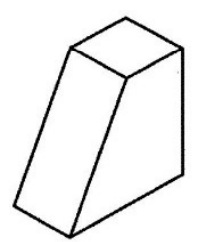

D

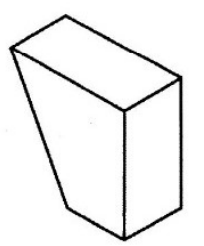

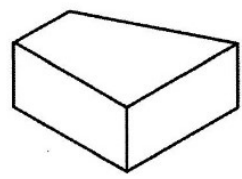

Notice that the given rotation in this example is more complex. The correct answer for this example is B. 
- DO NOT make any marks in this booklet.

- Mark your answers on the separate answer sheet.

- You will be told when to begin. 
1
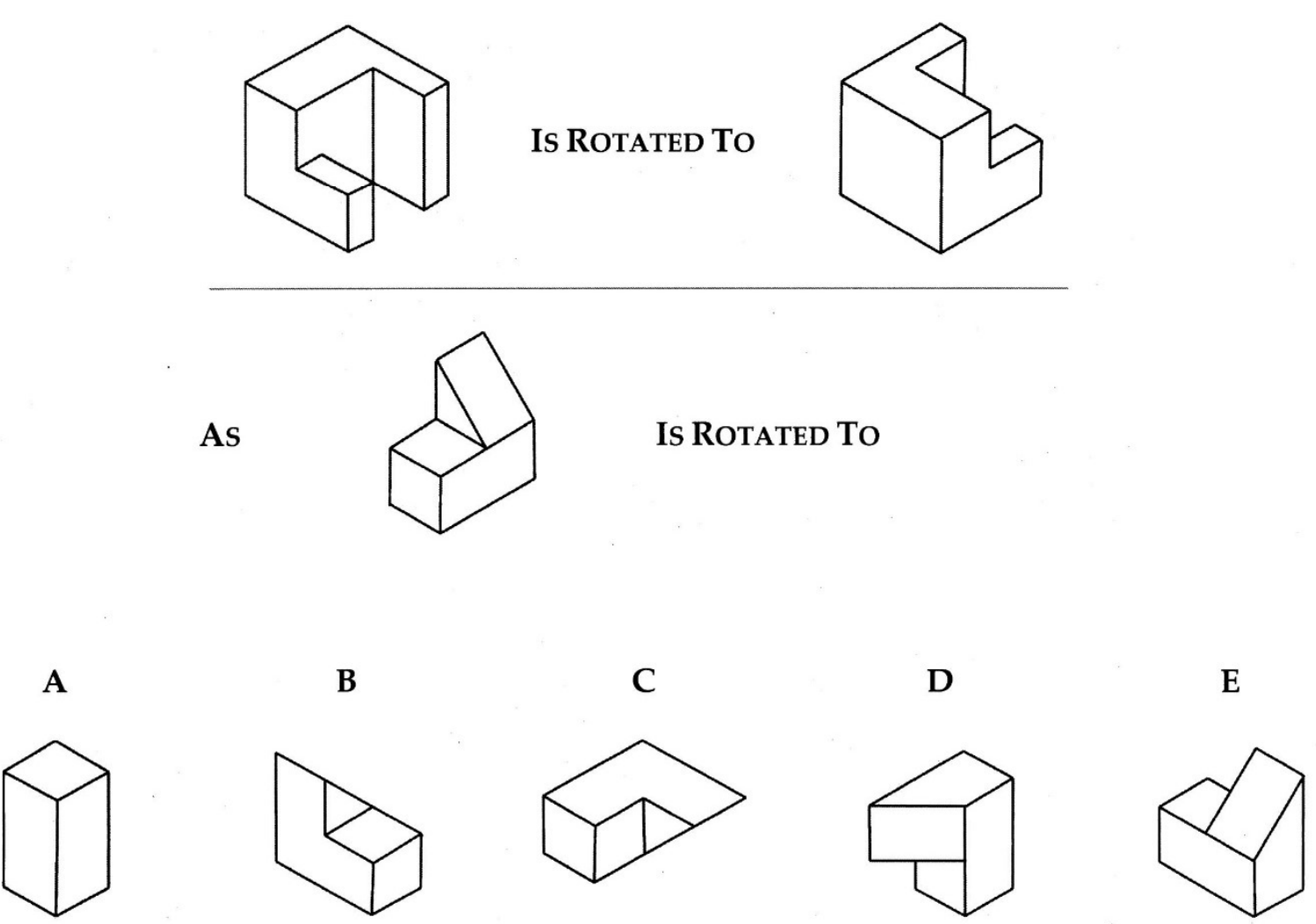
2

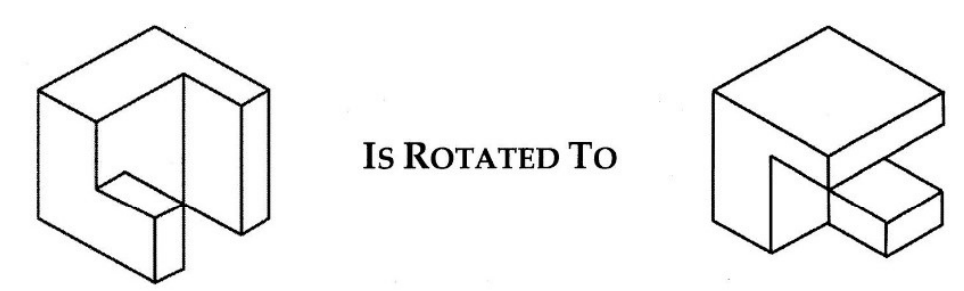

As Is Rotated To

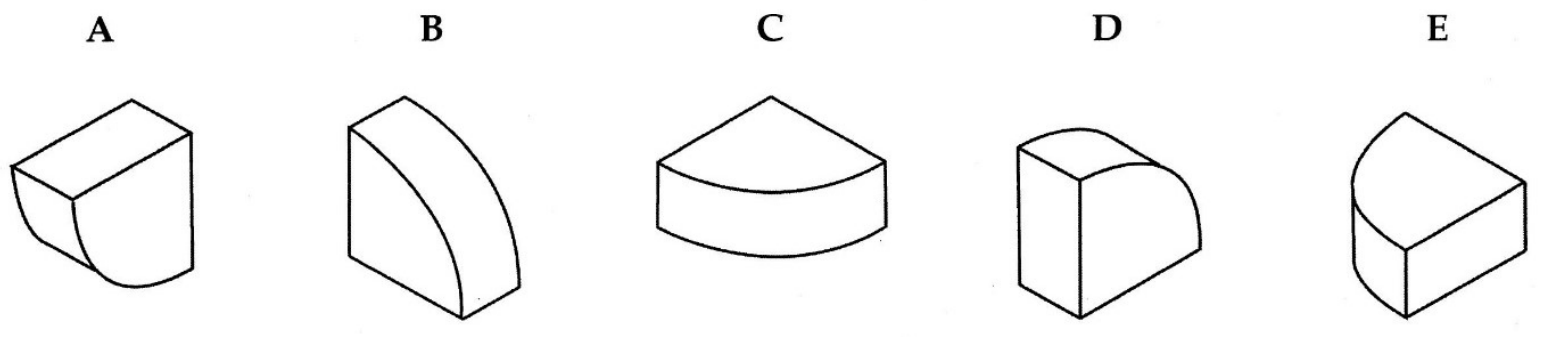


3
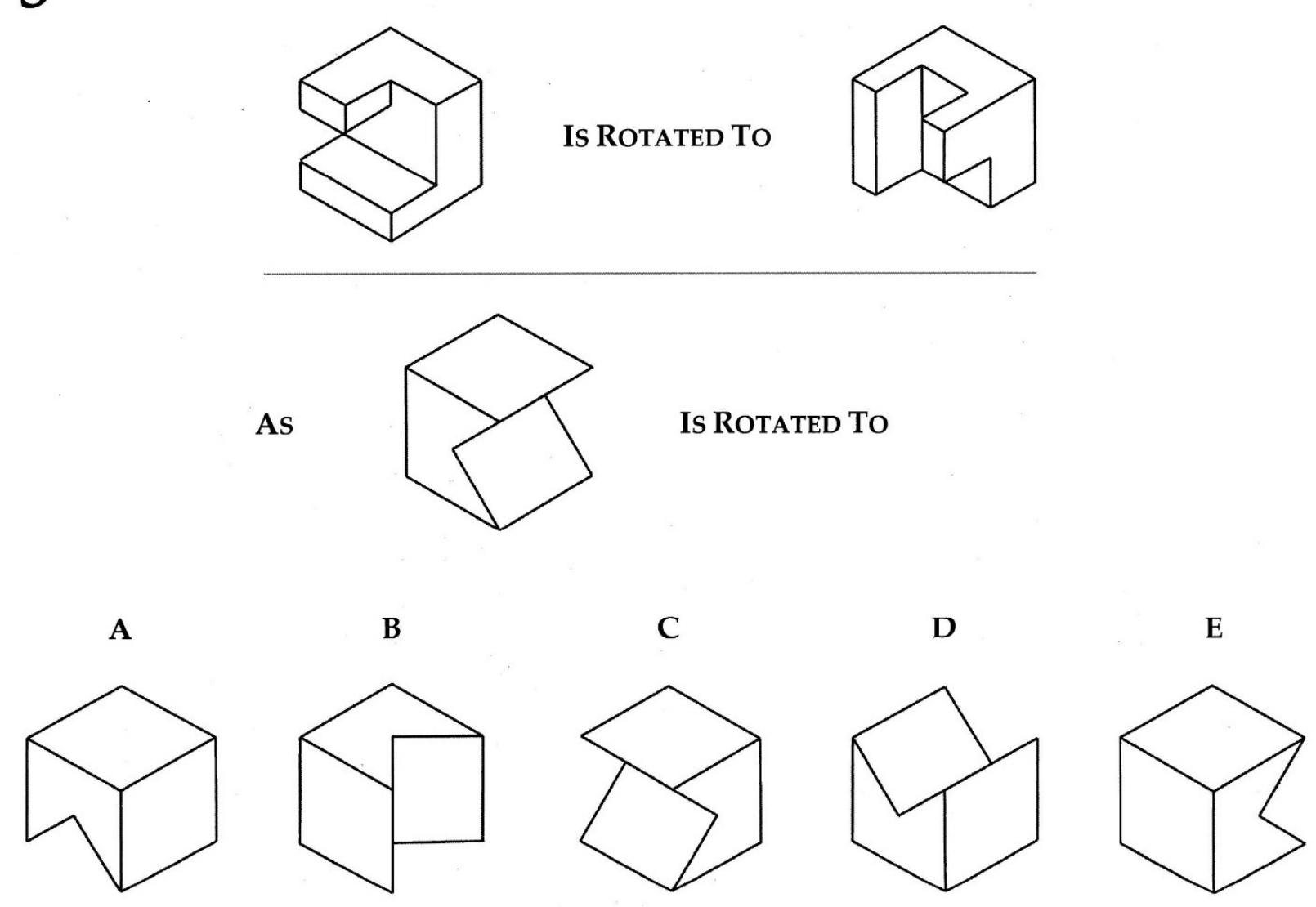
4

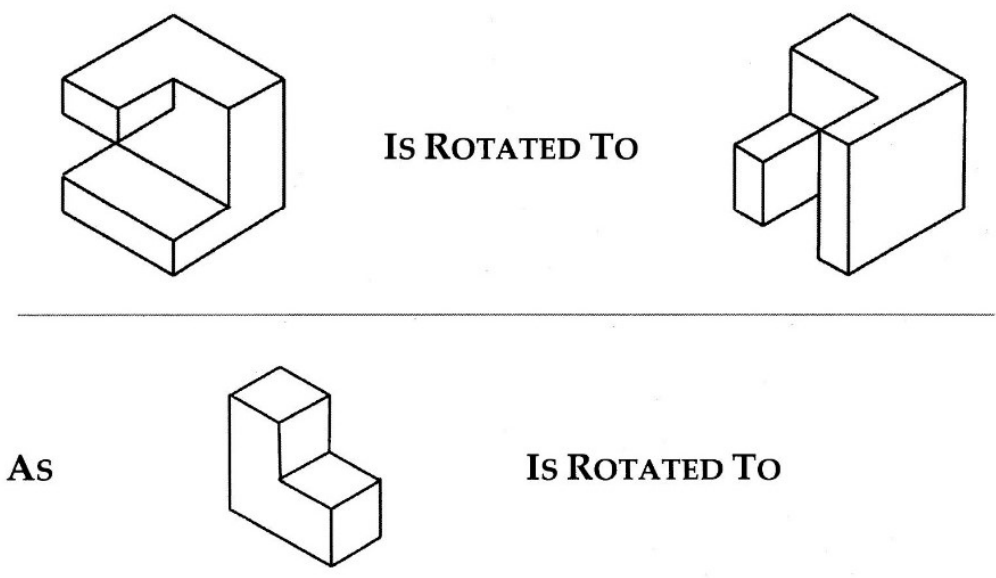

A

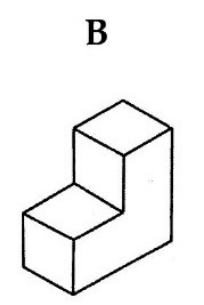

(O)

(O)

E<smiles>C1C2CC3CC1CC(C2)C3</smiles>

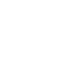


5
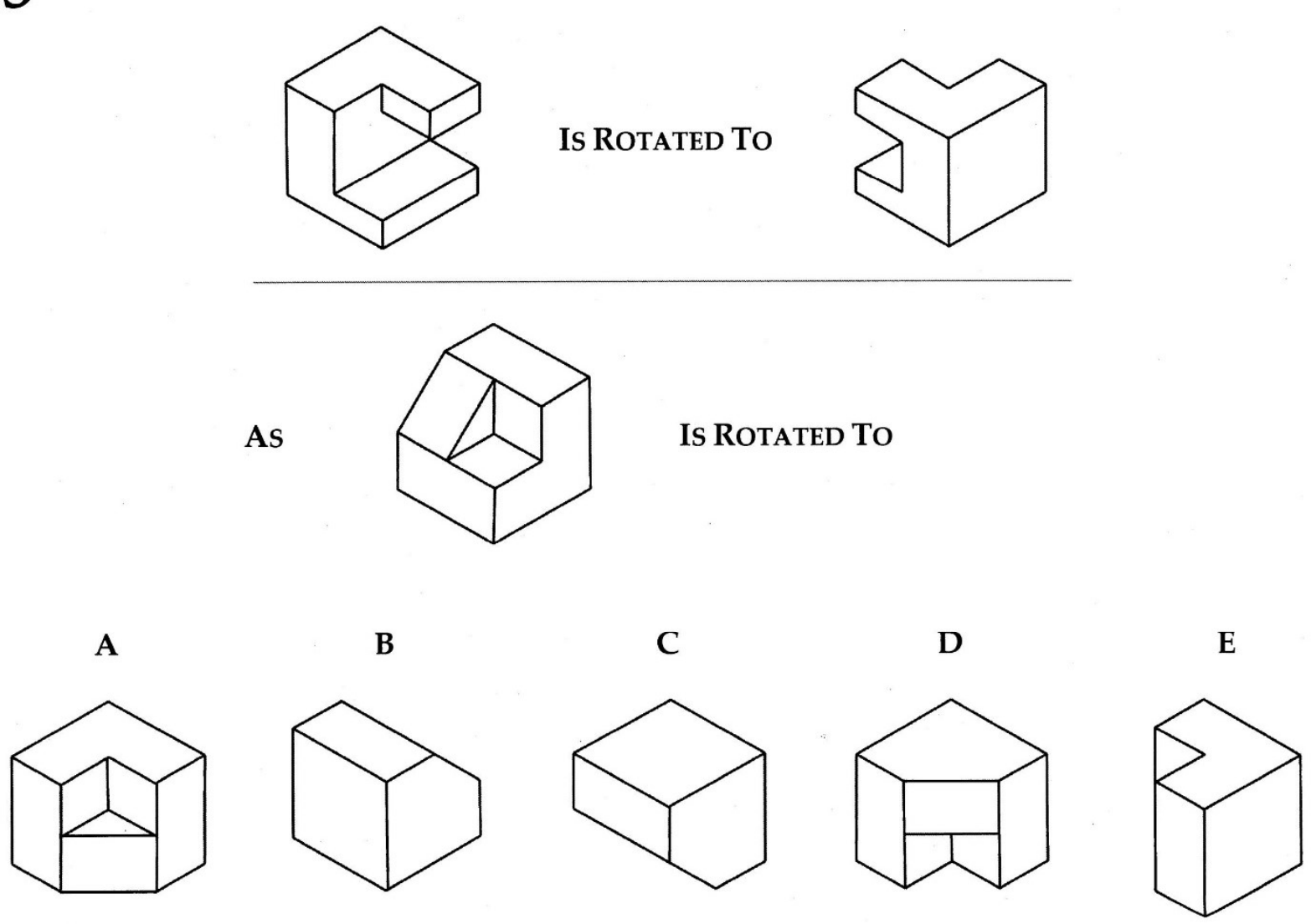
144

6
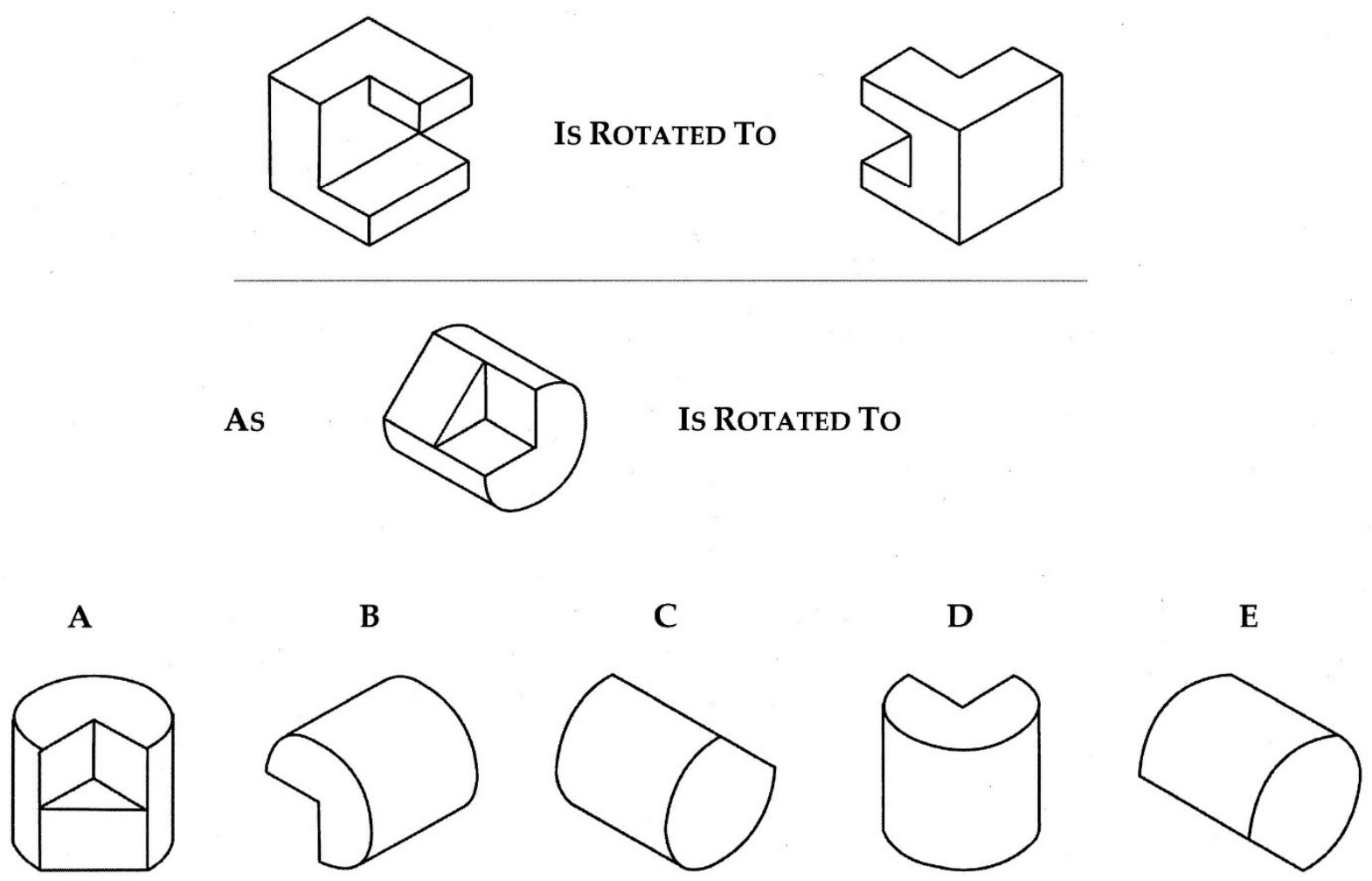
7

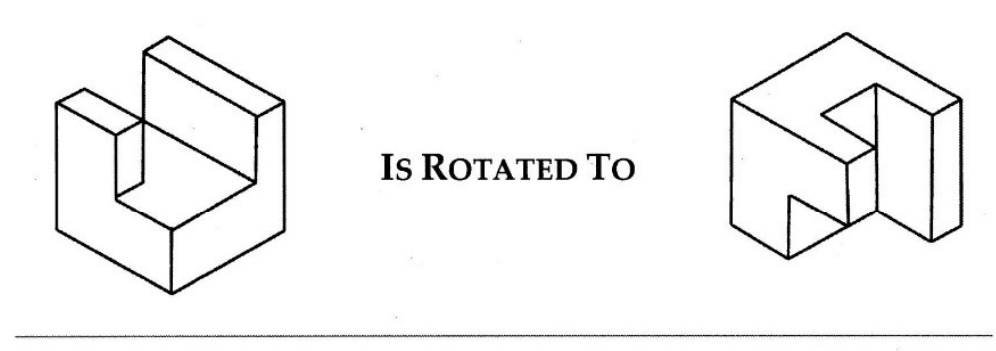

Is Rotated To

A

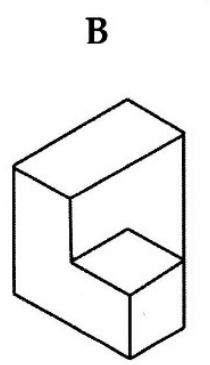

C
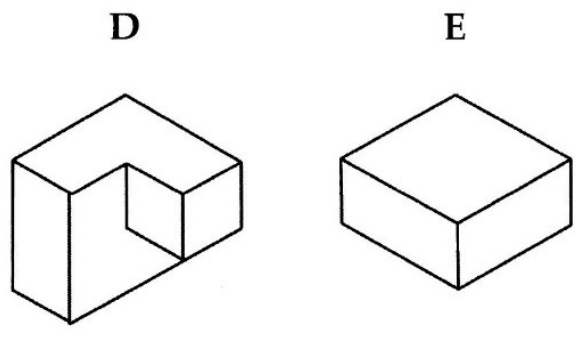
8
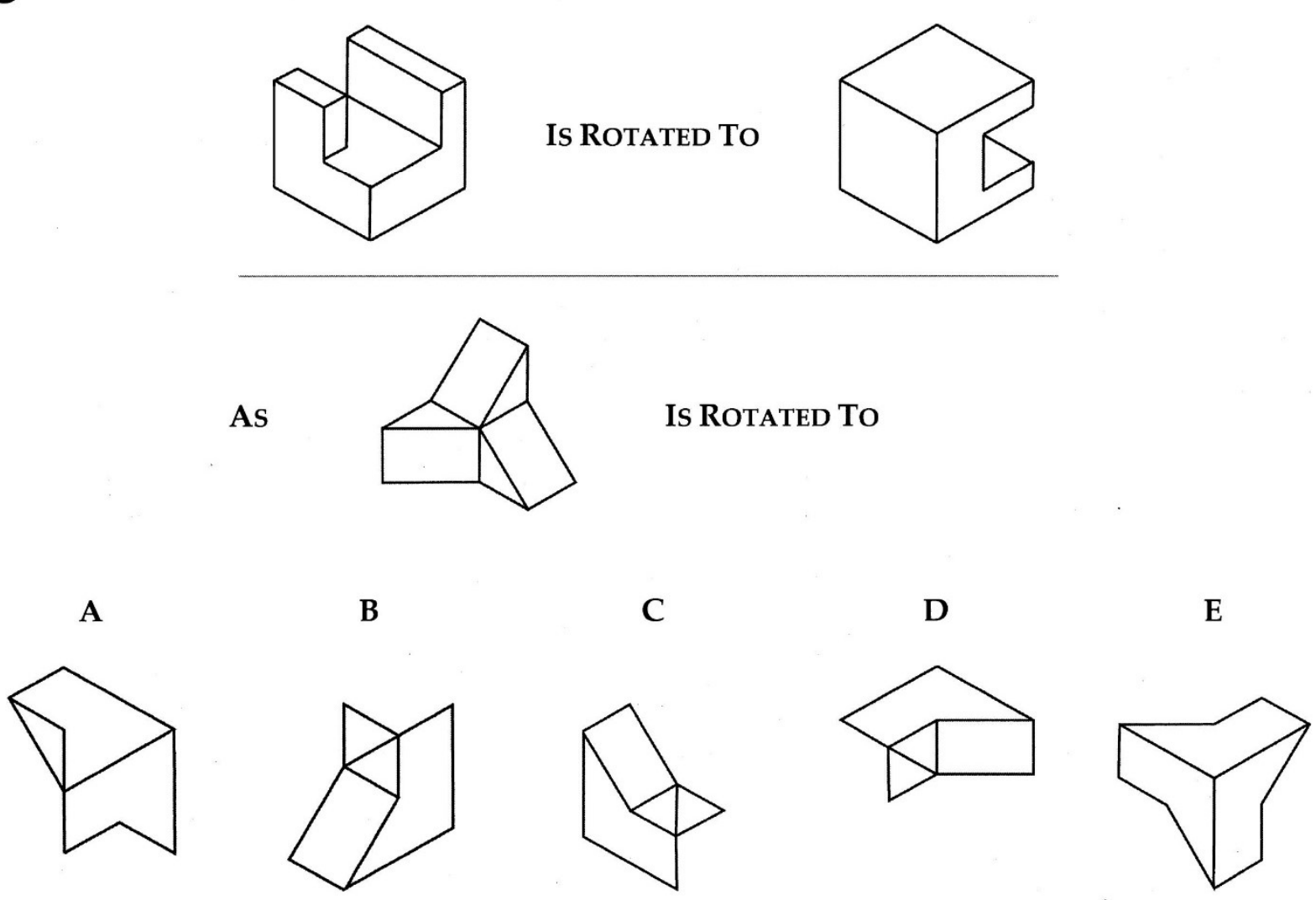
9

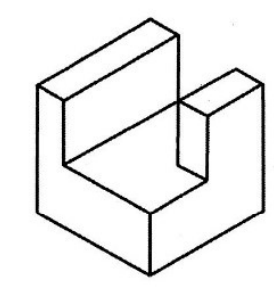

Is ROTATED To
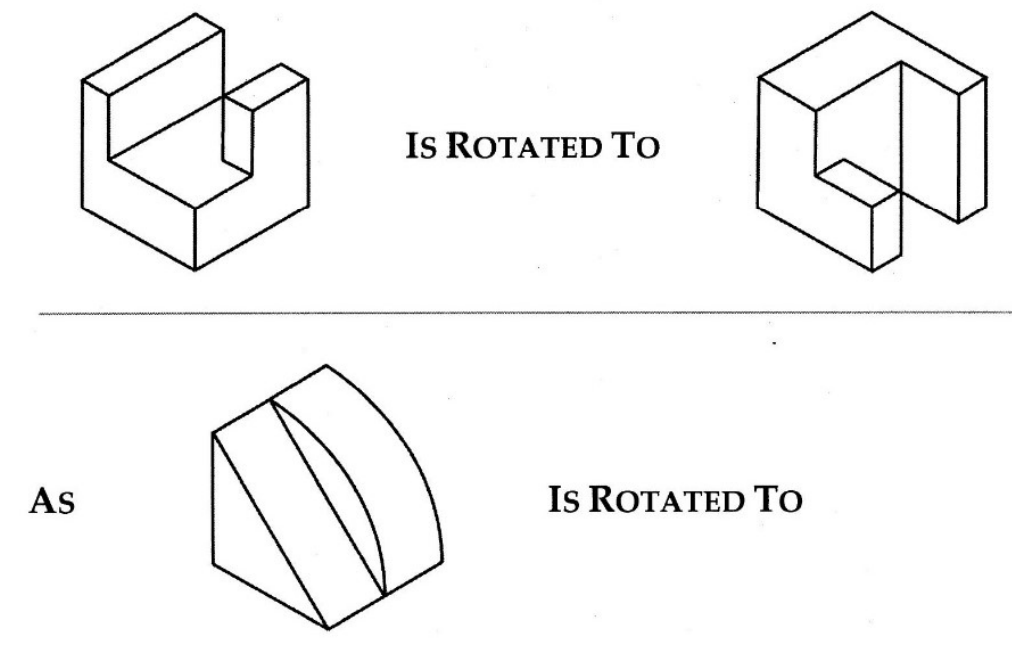

Is Rotated To
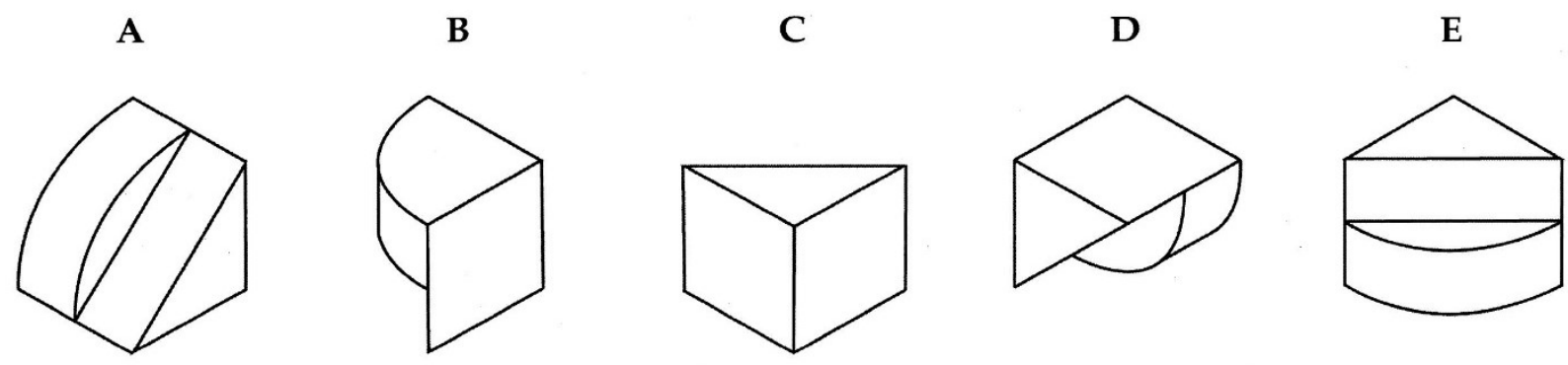
10

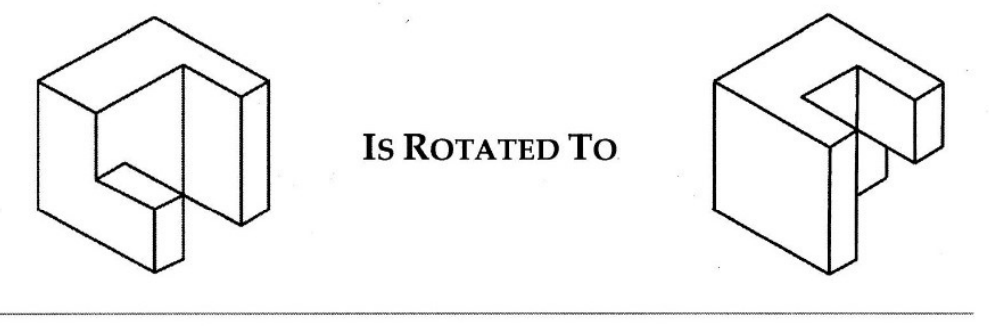

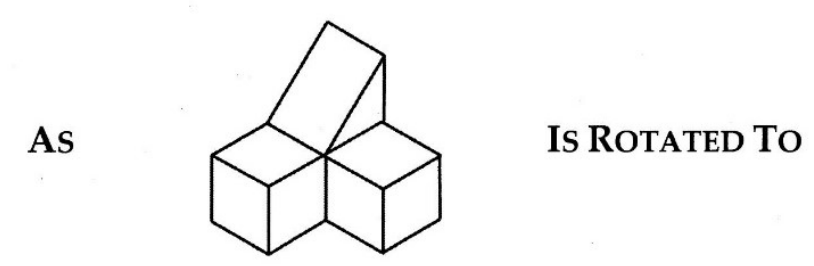

A

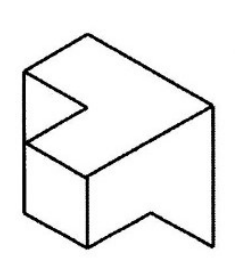

B

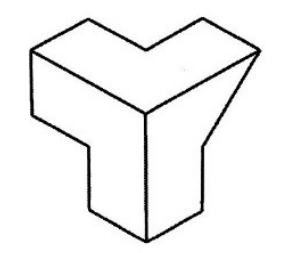

C

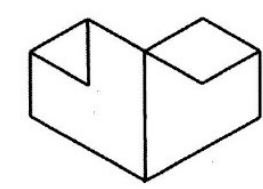

D

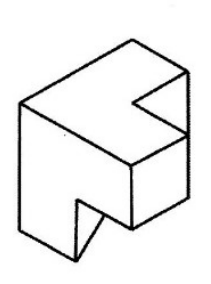

E

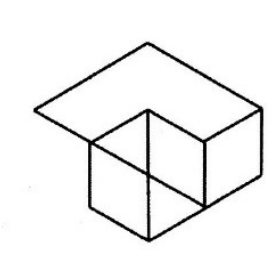


11
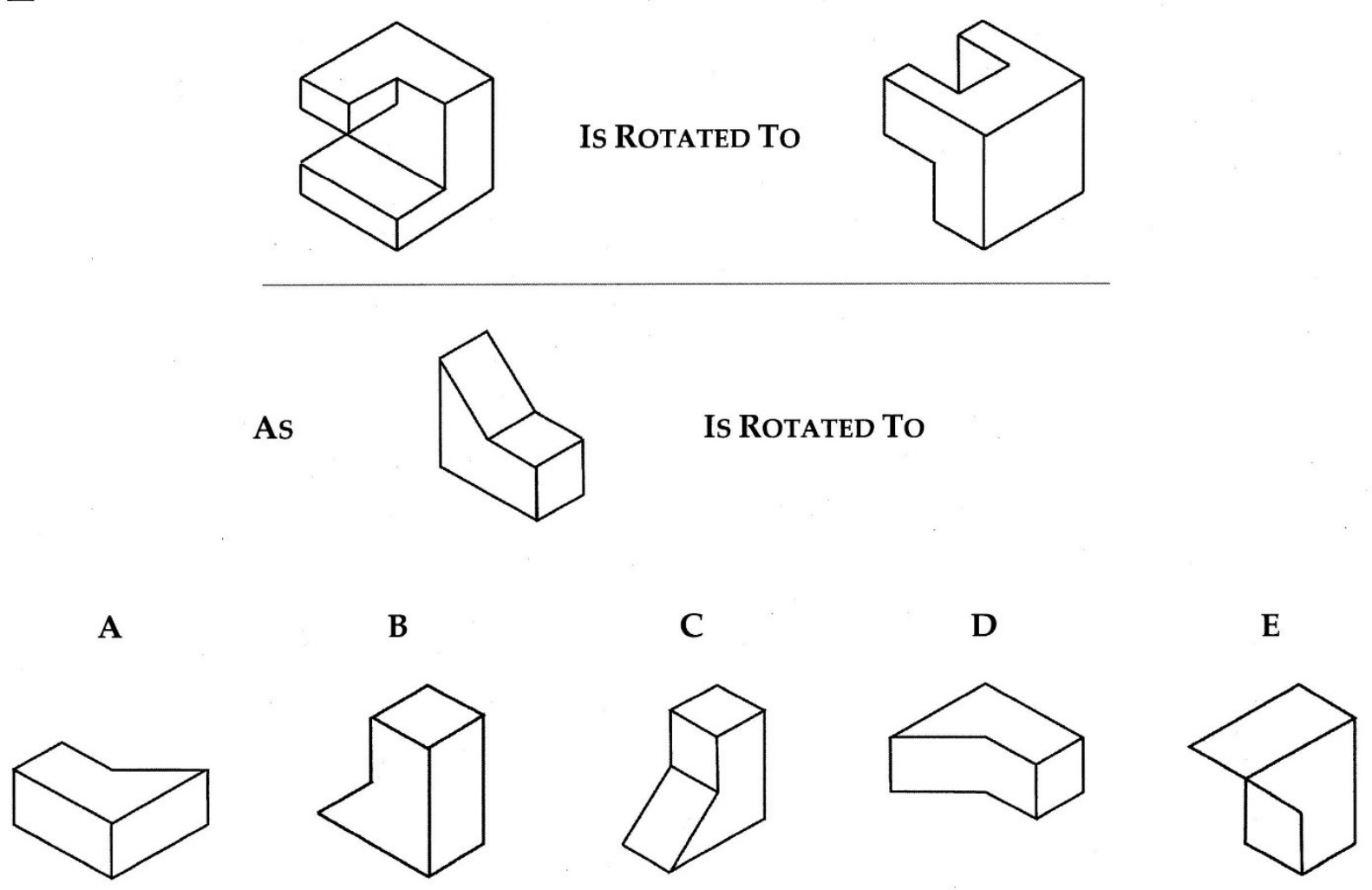
12
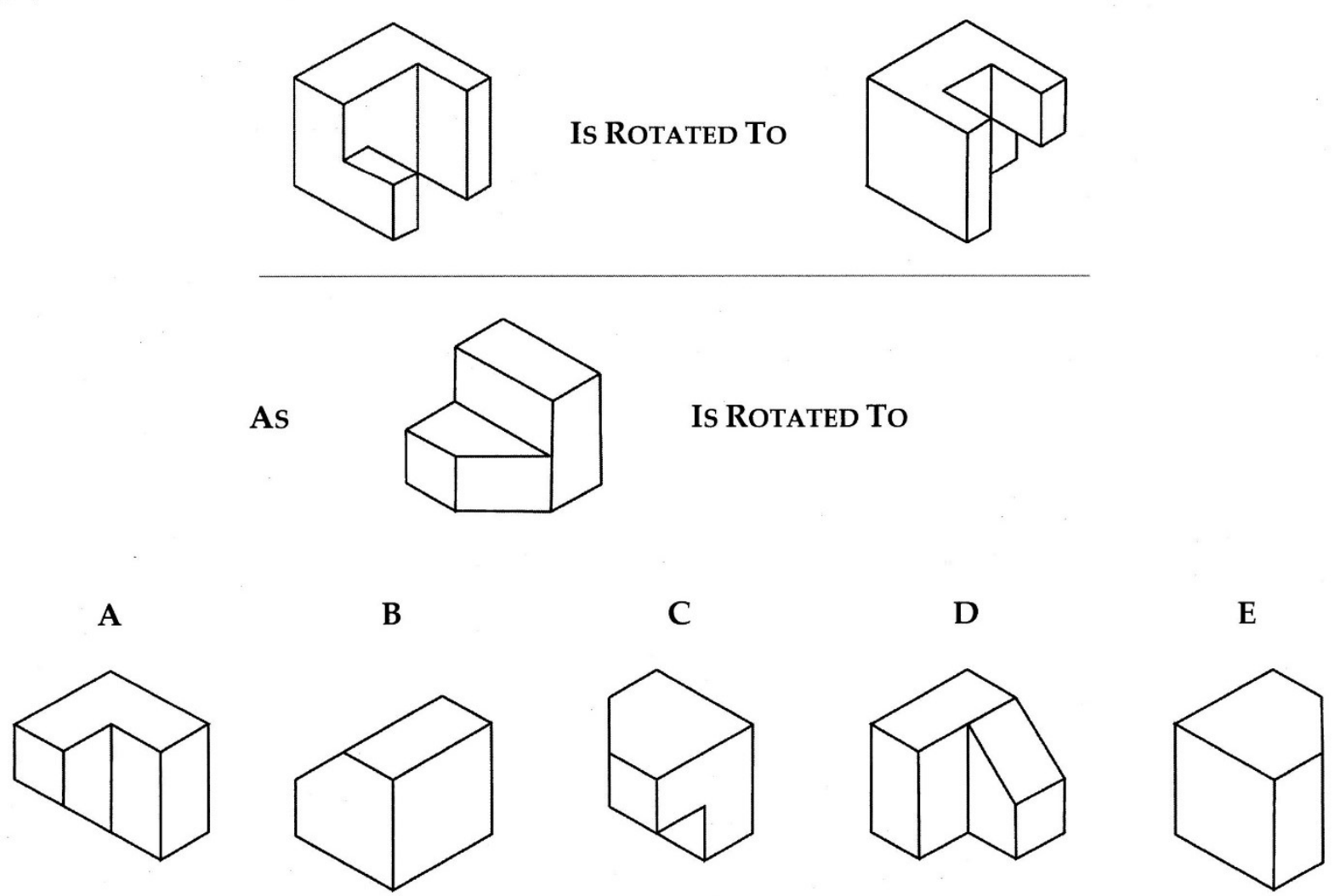
13
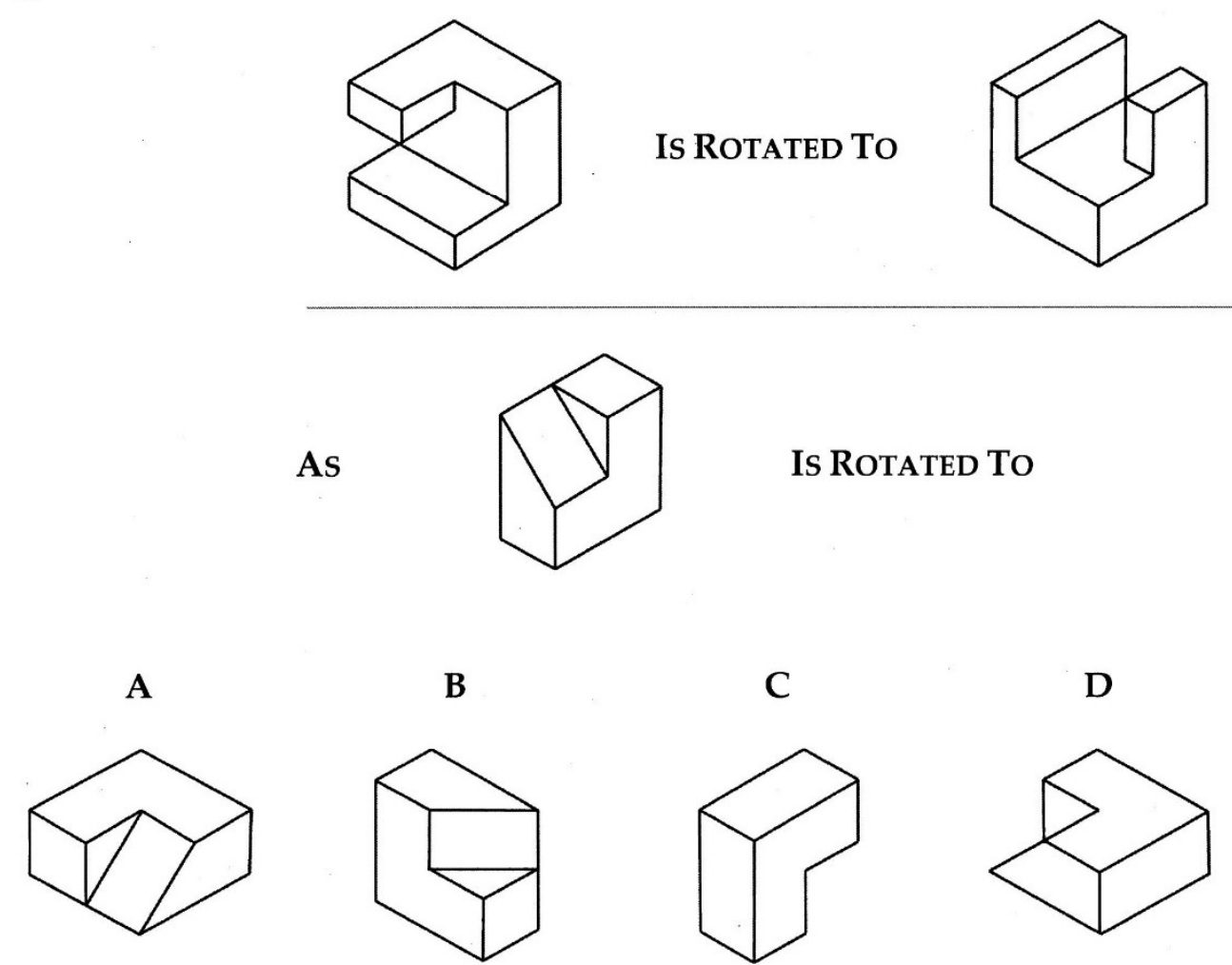

E

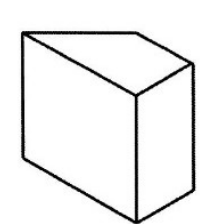


14

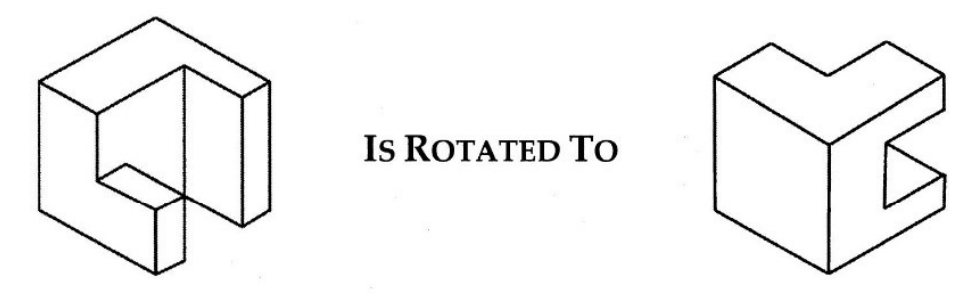

As

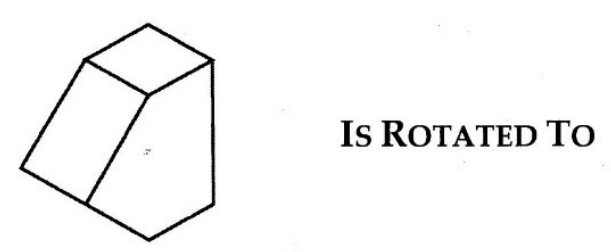

A

B

C

D

E
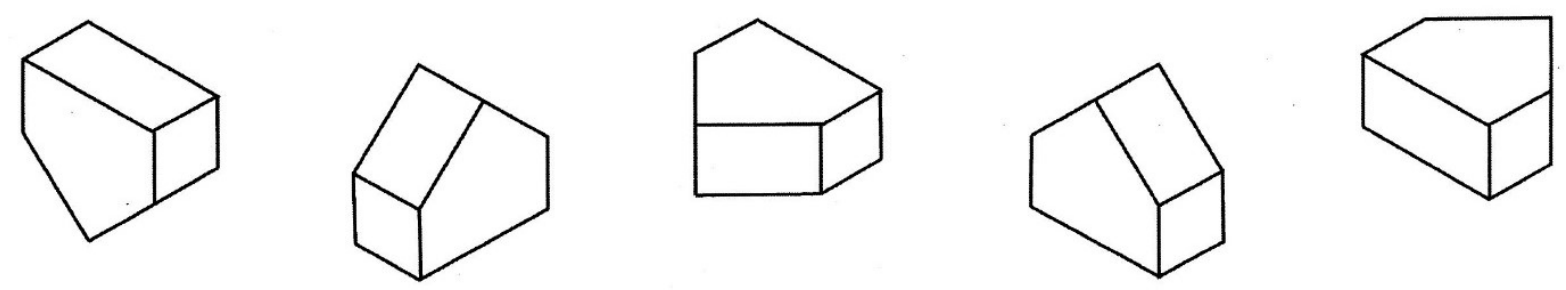
15
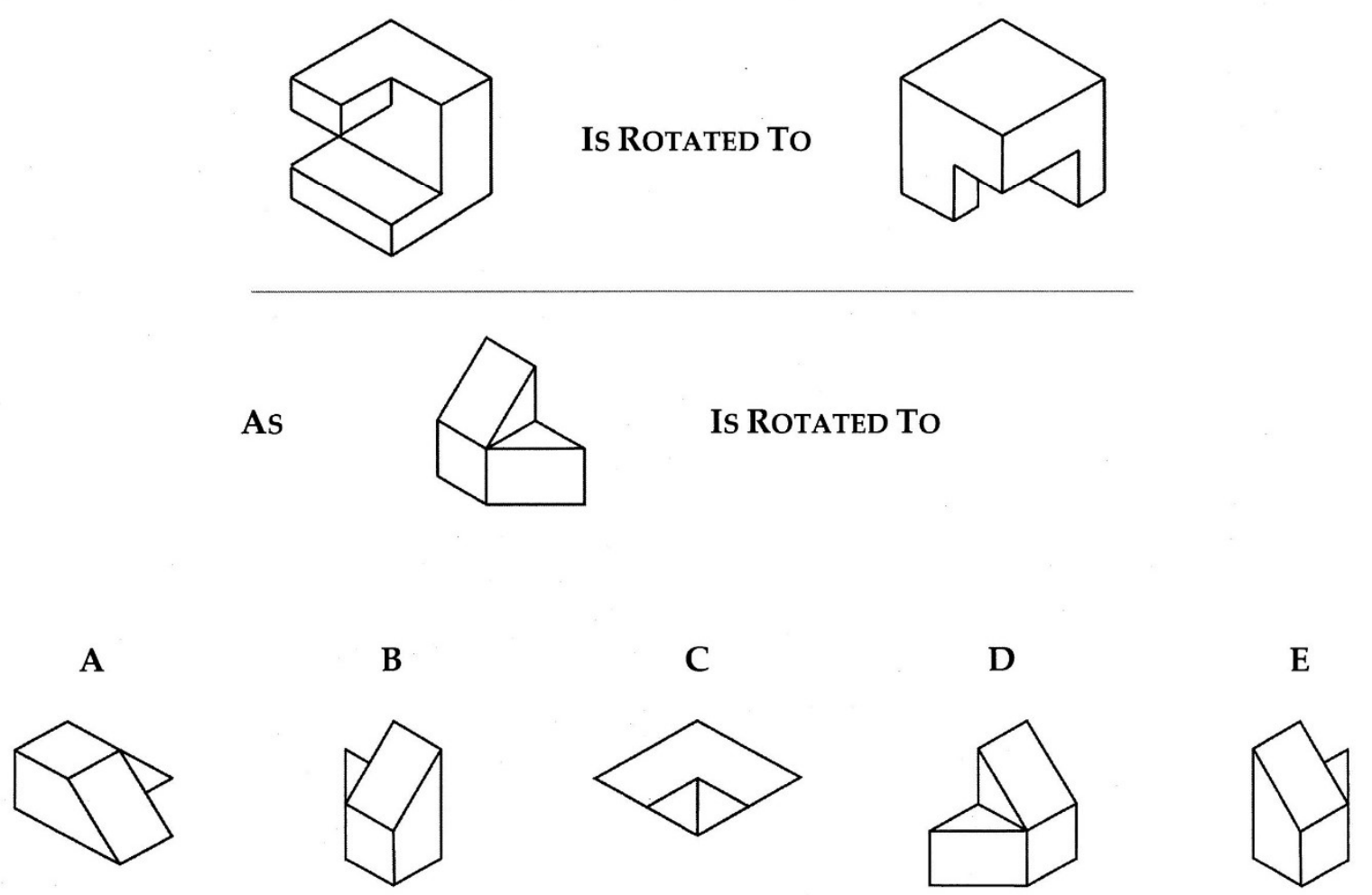


\section{6}
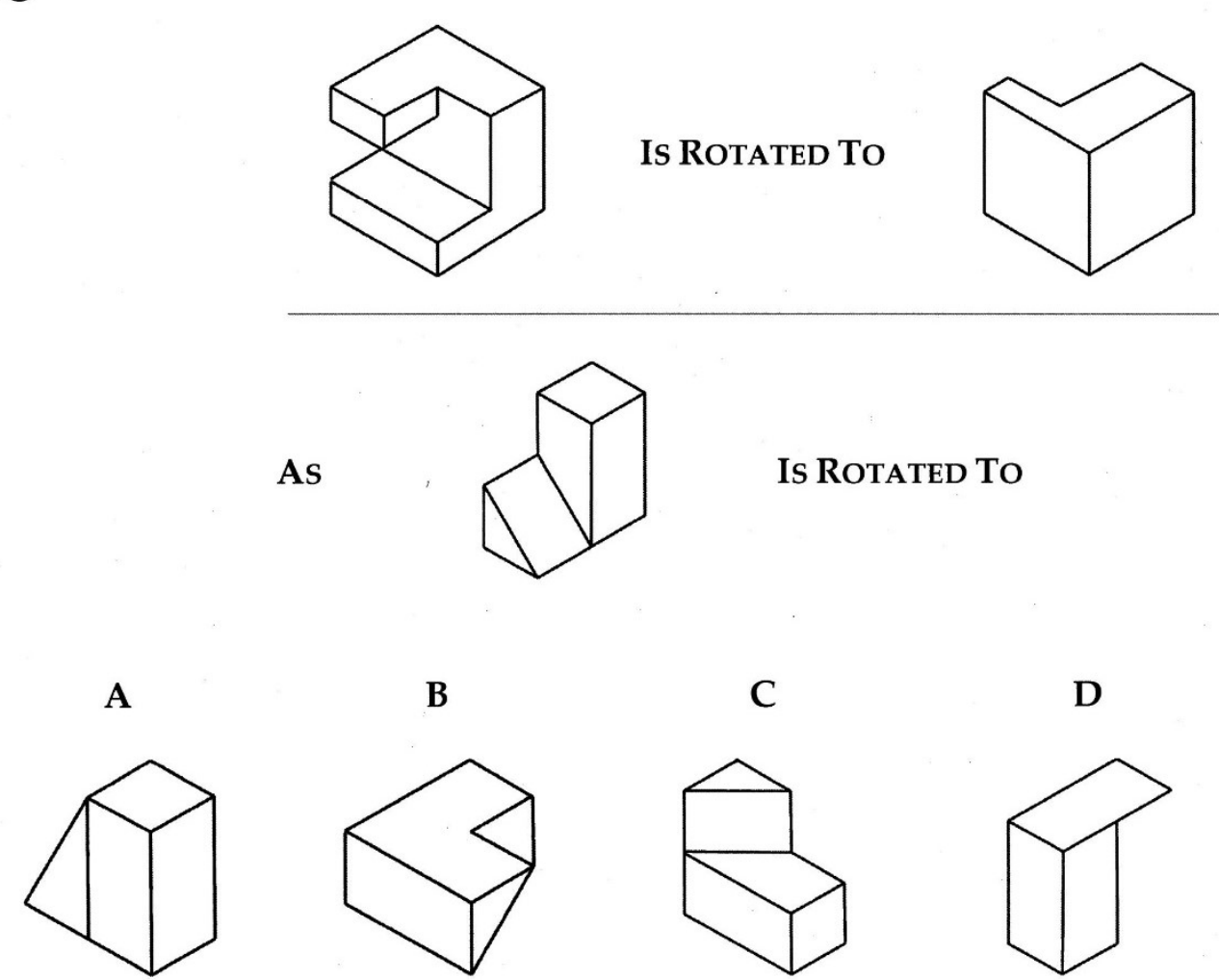

E

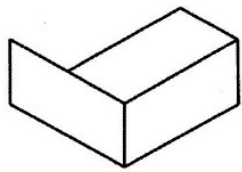




\section{7}
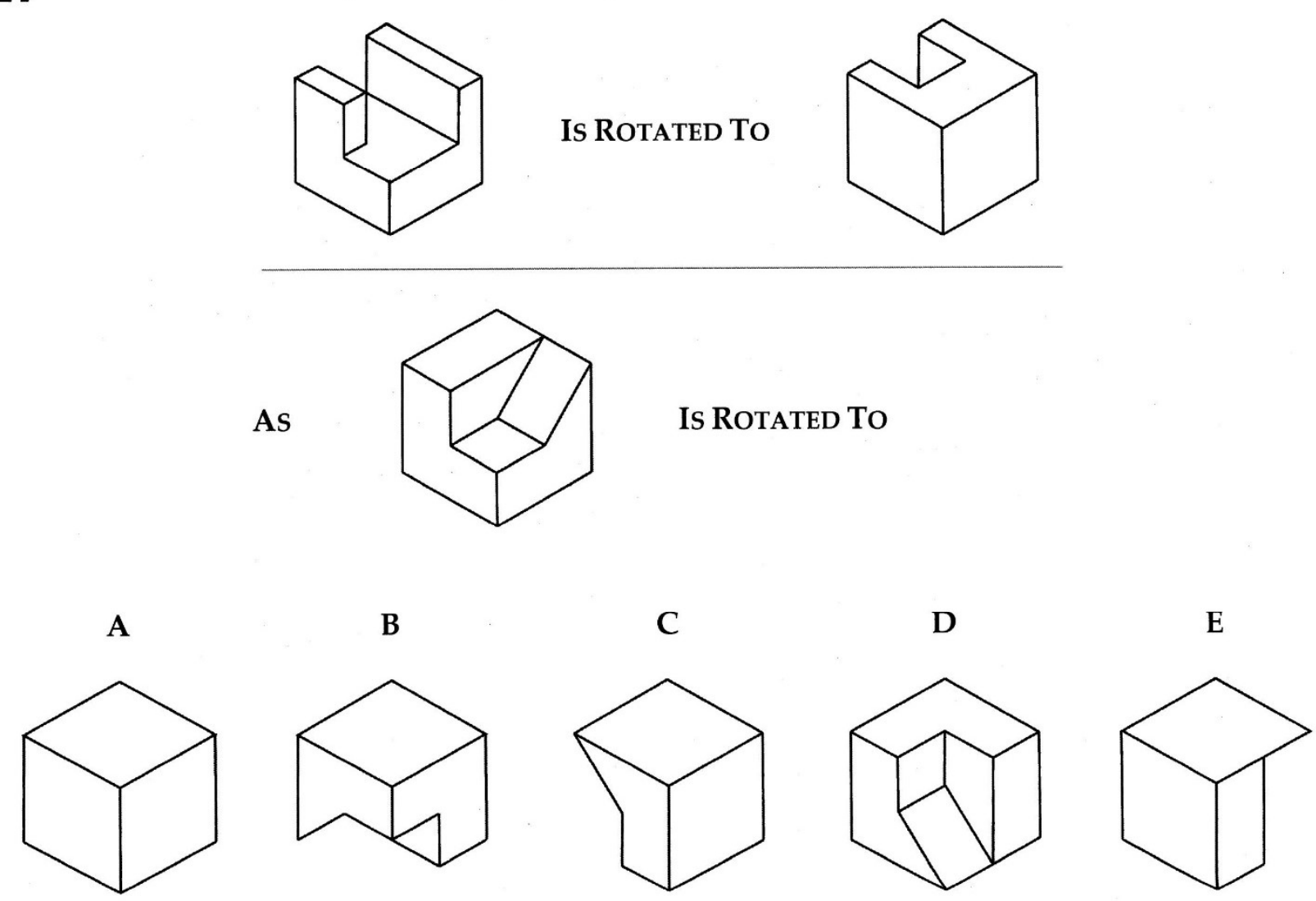
18
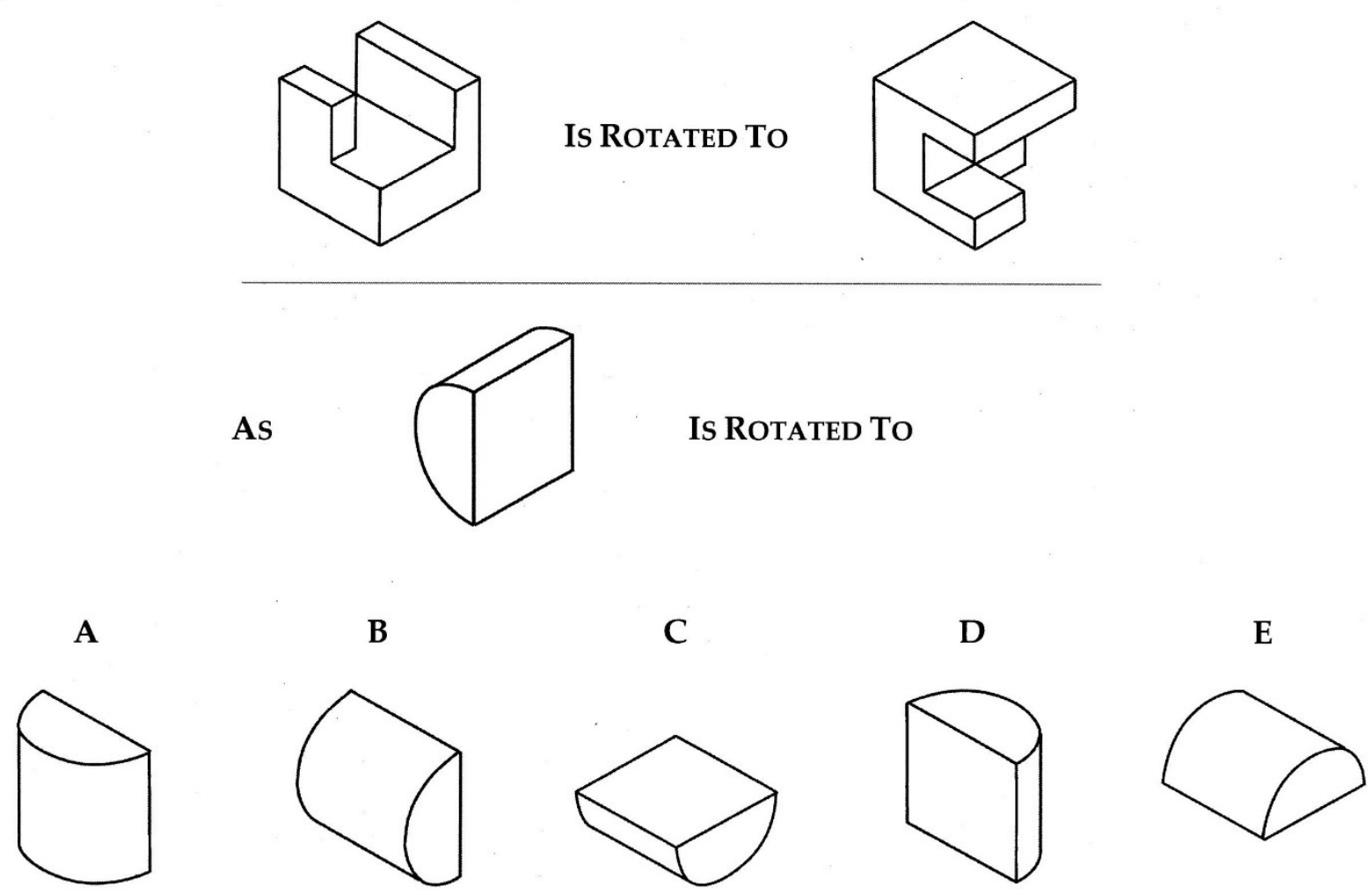
19
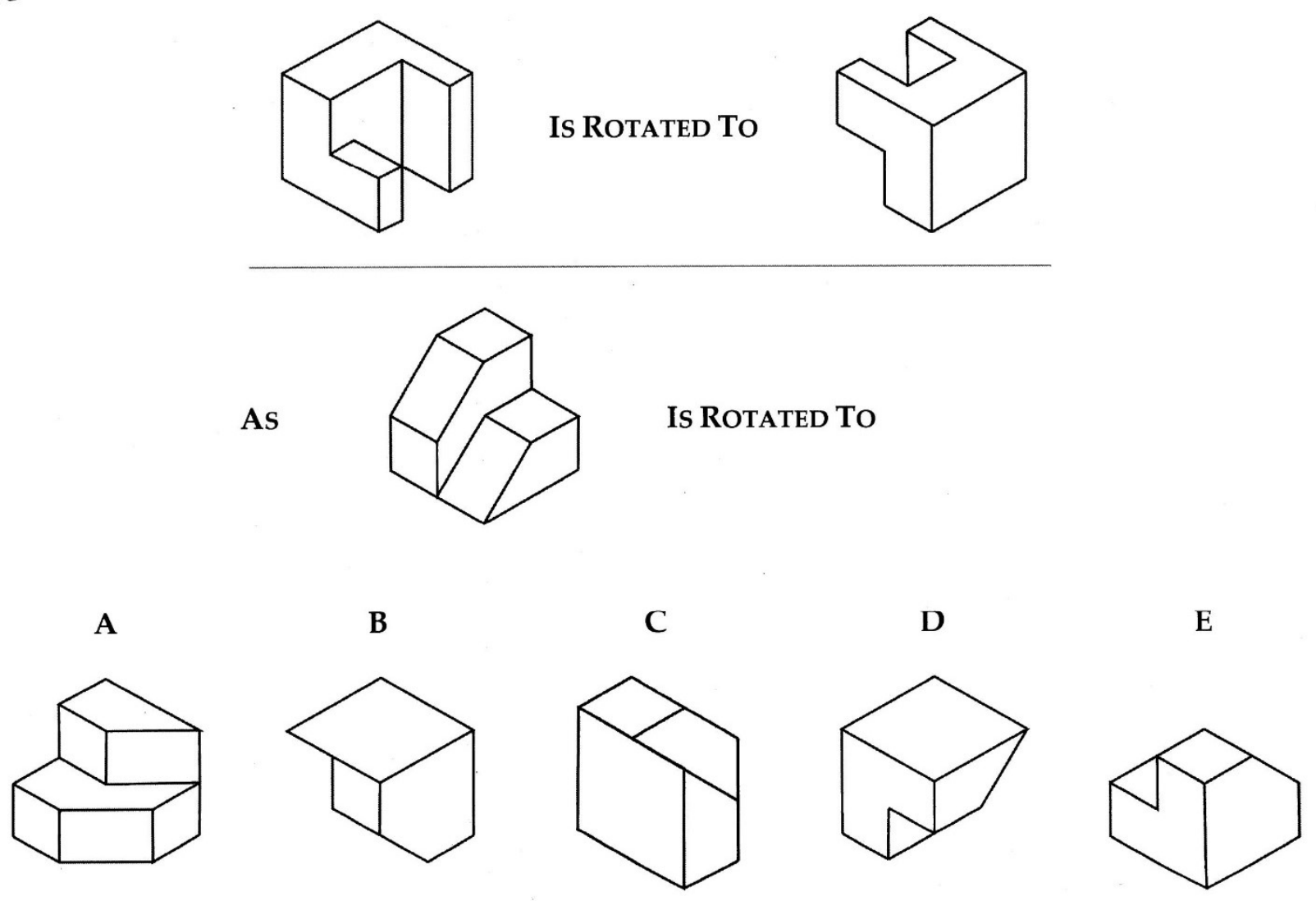
20
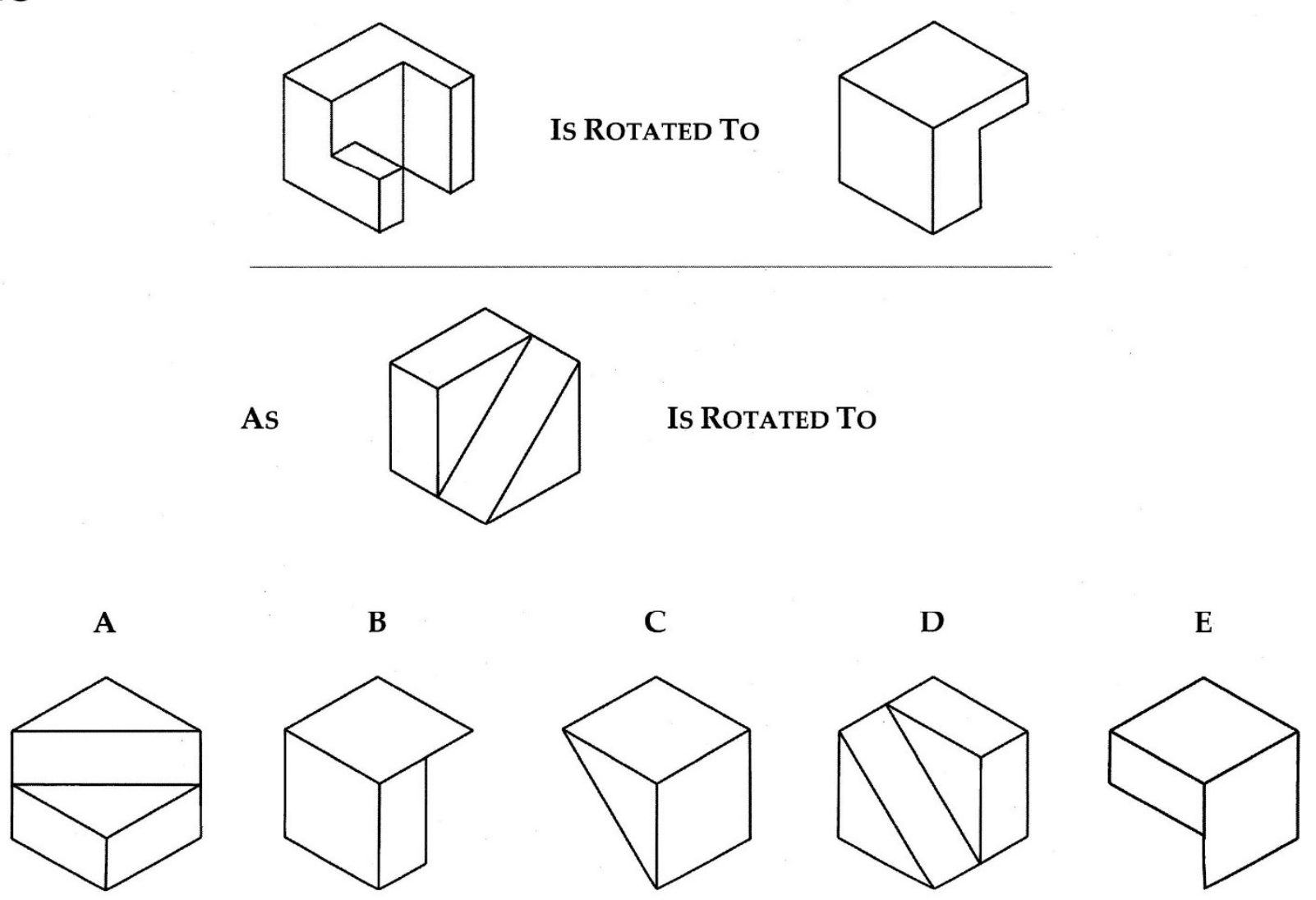
21
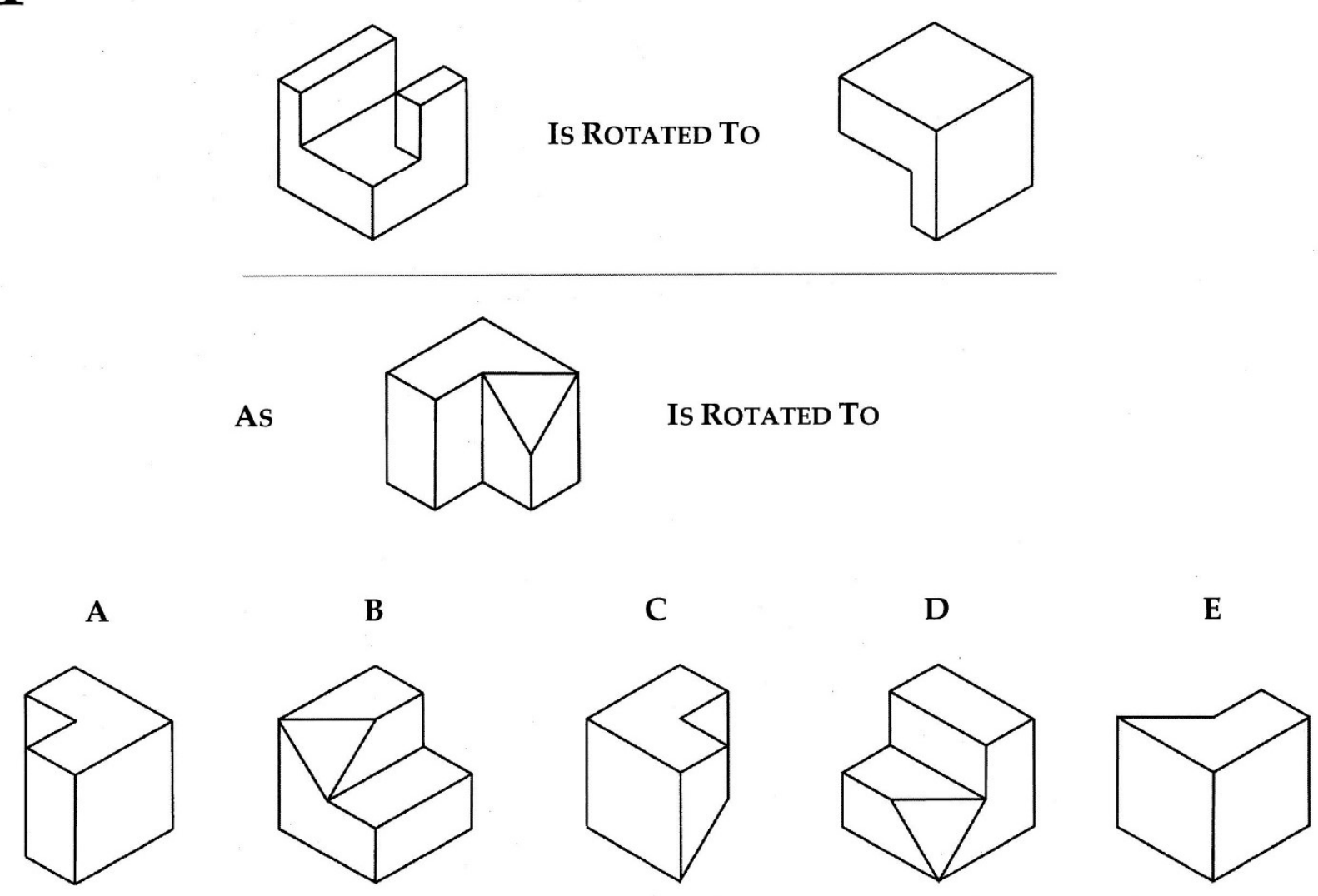
160

22

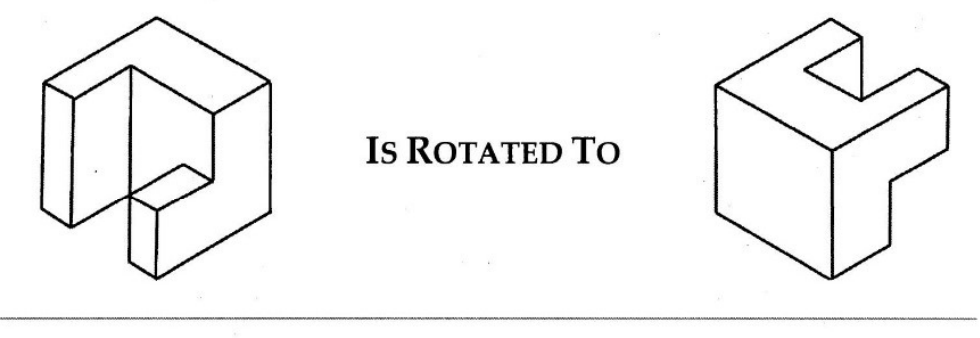

As

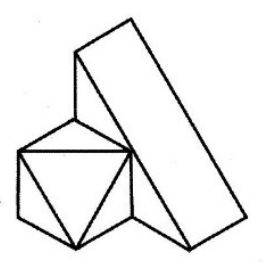

IS ROTATED TO

A

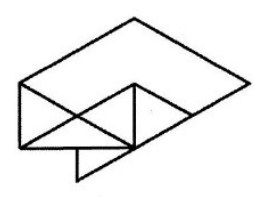

B

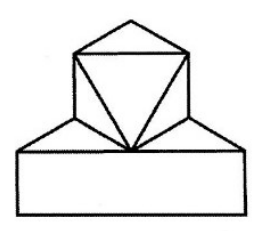

C

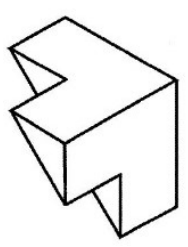

D

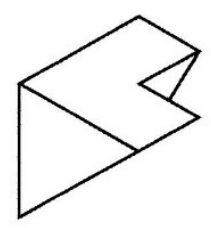

E

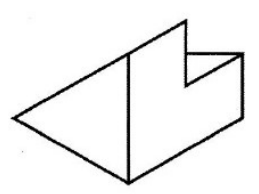


23
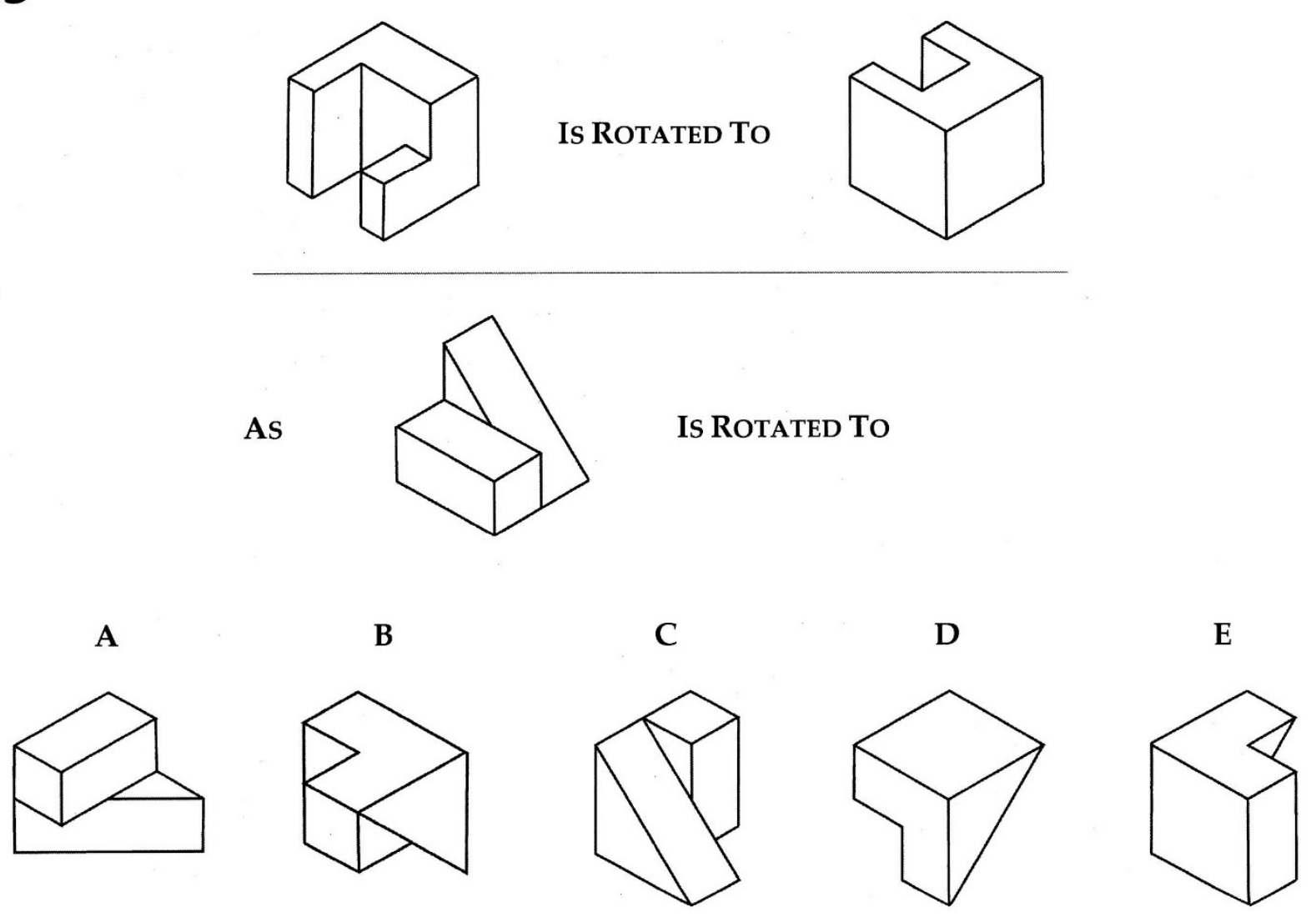


\section{4}
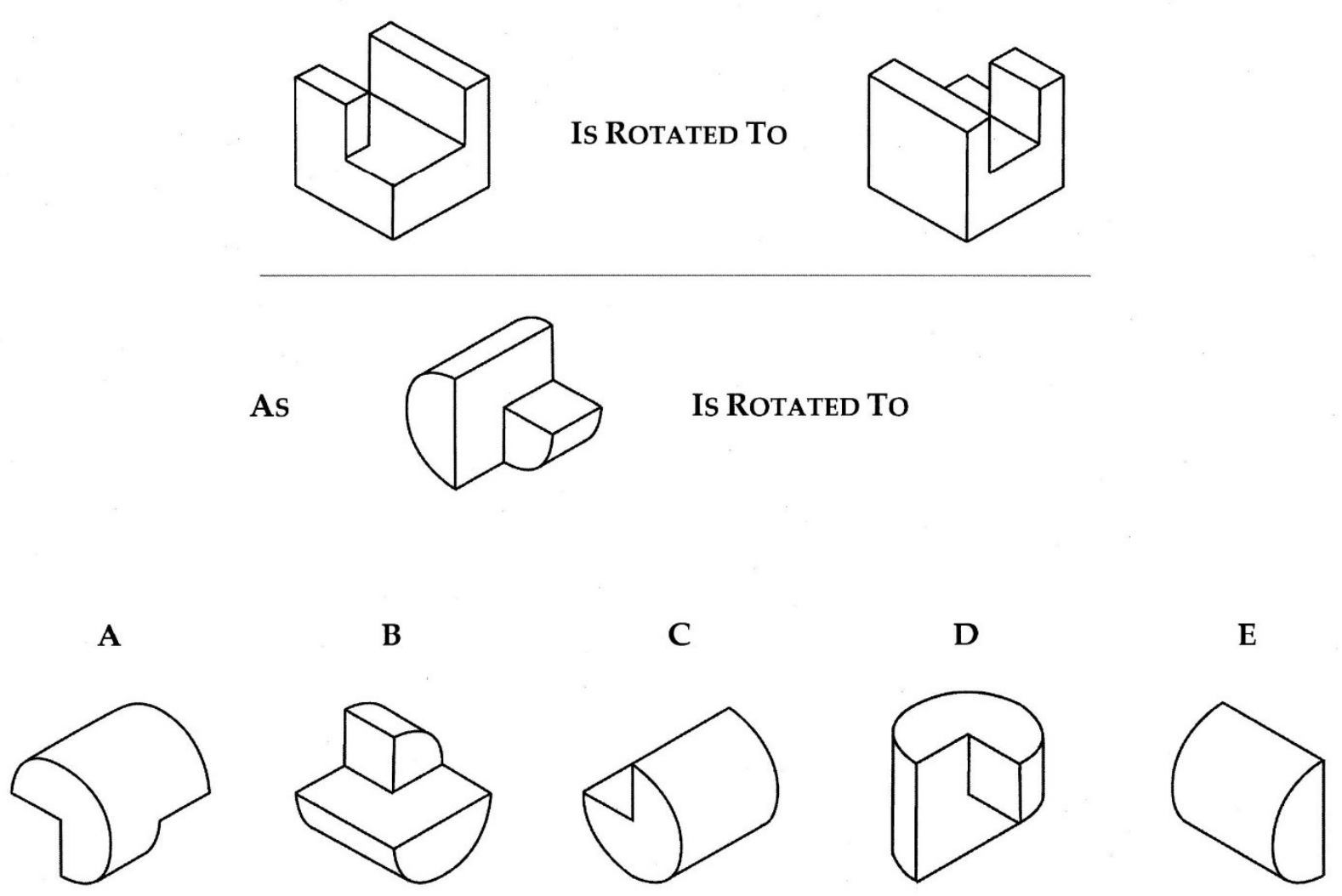
25
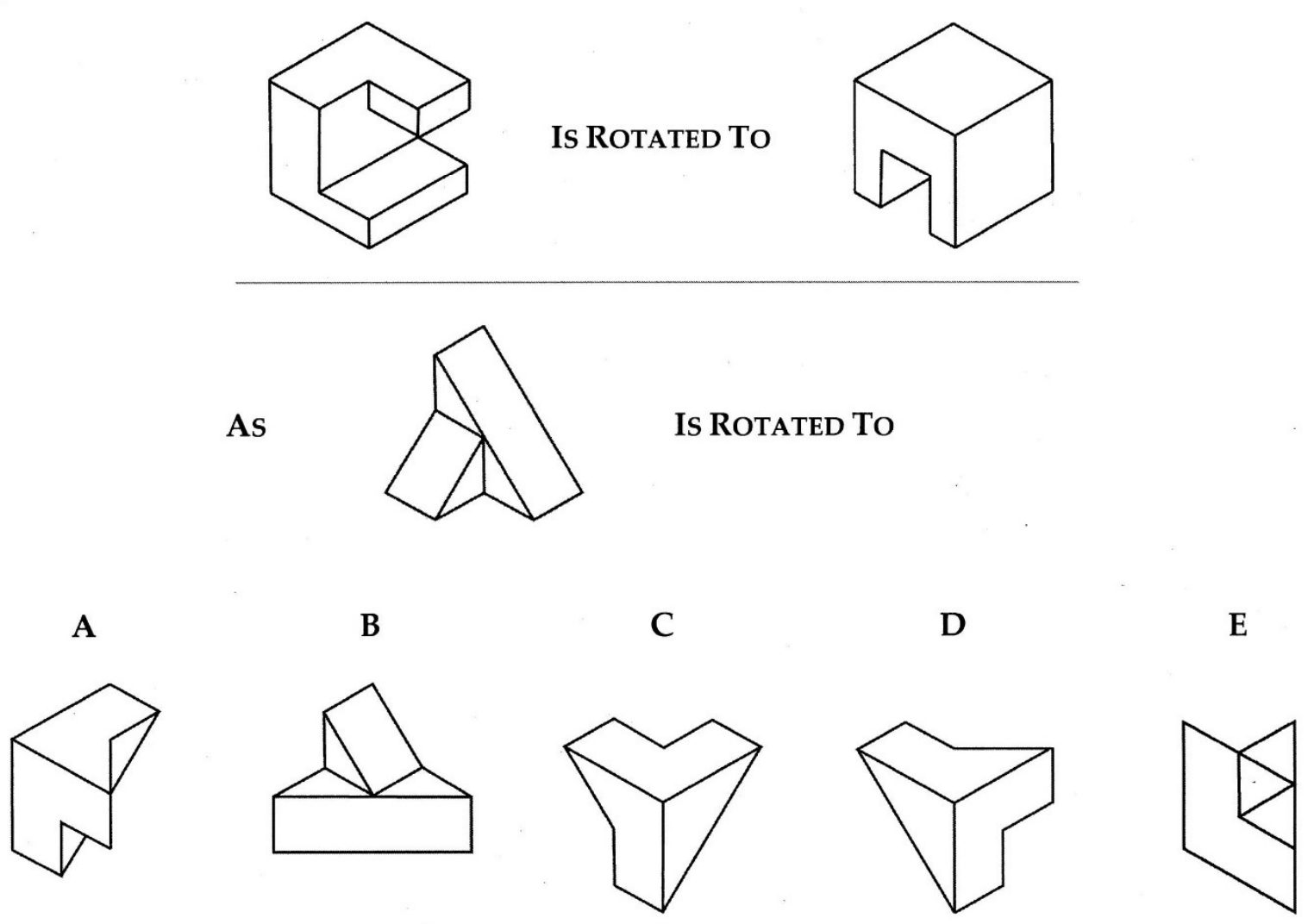


\section{6}
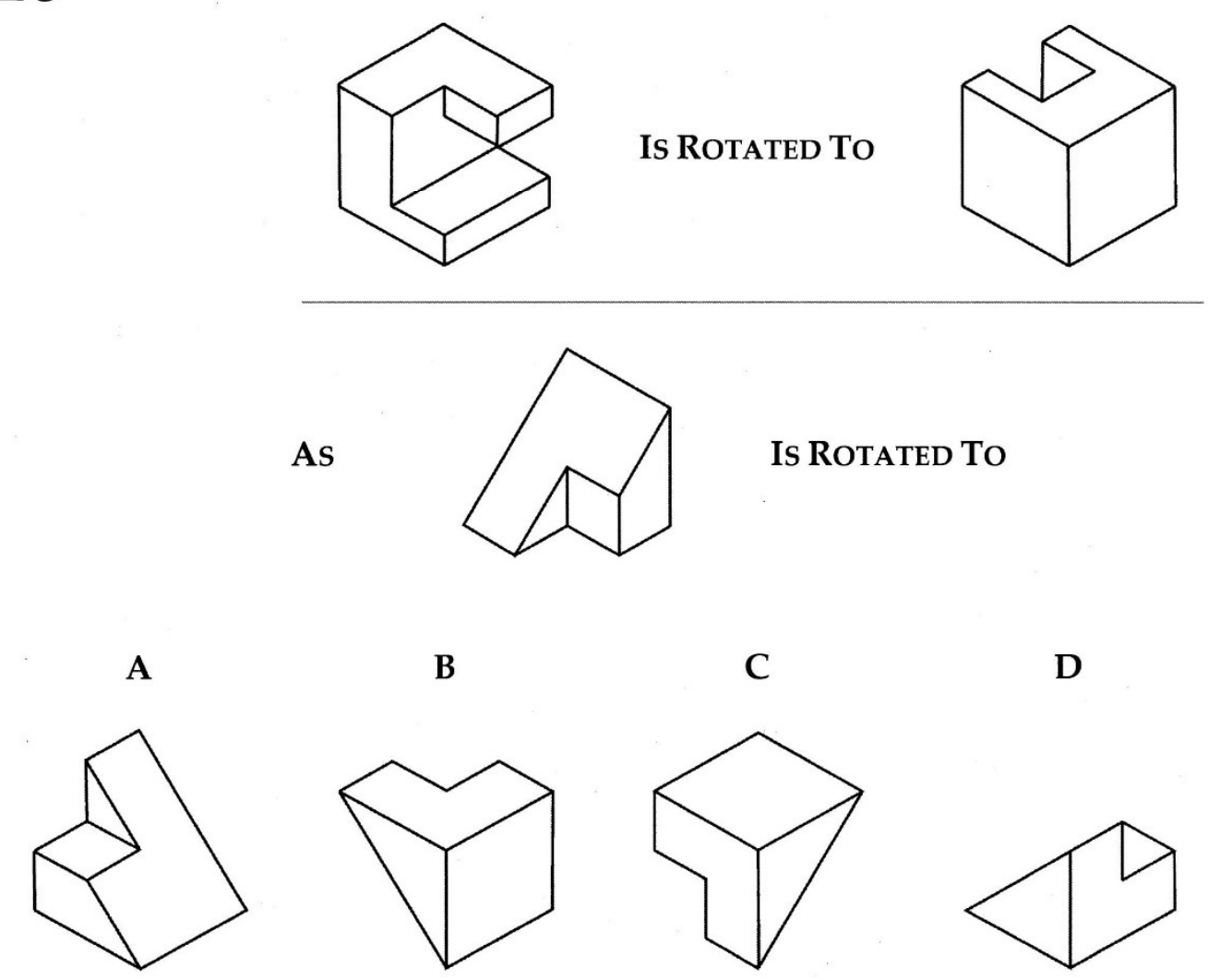

D

E 


\section{7}
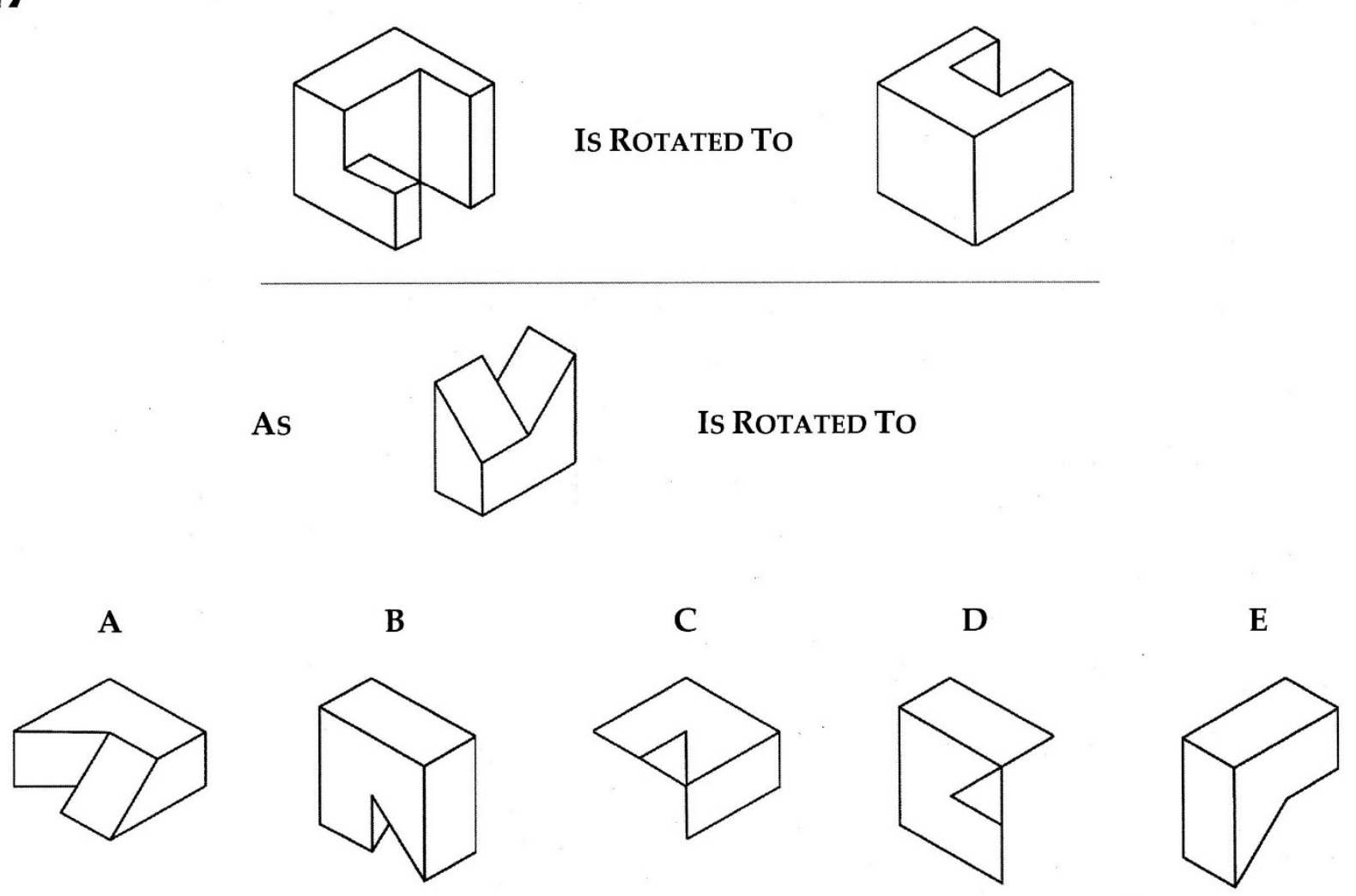


\section{8}

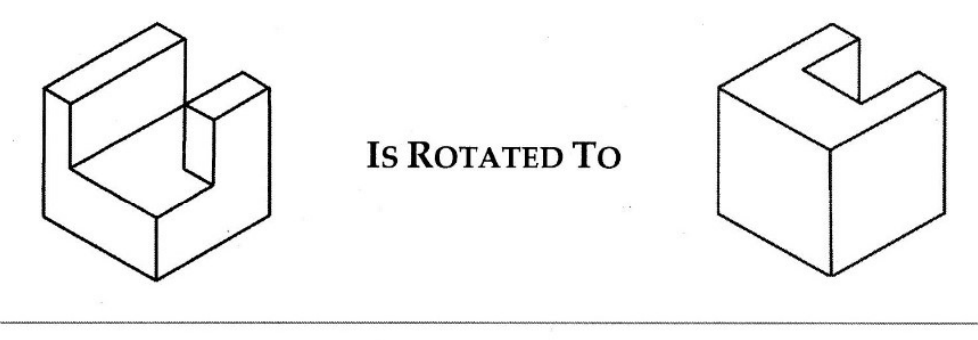

As
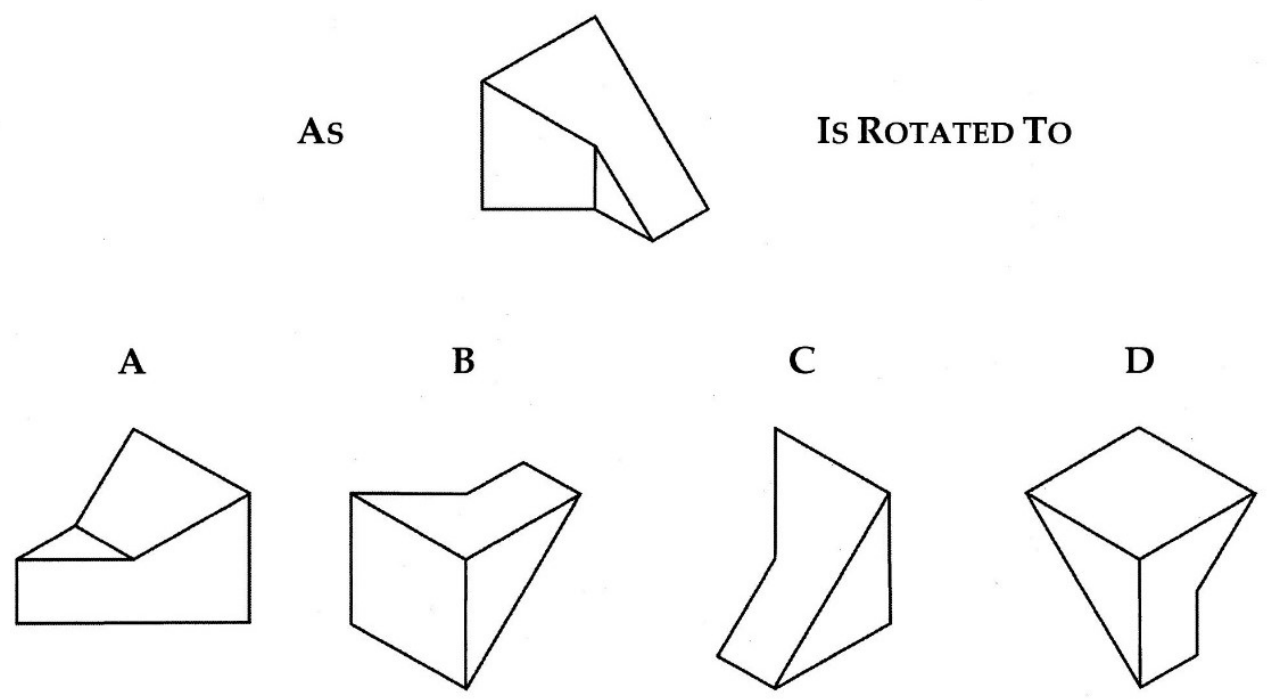

E

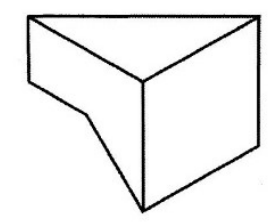


29
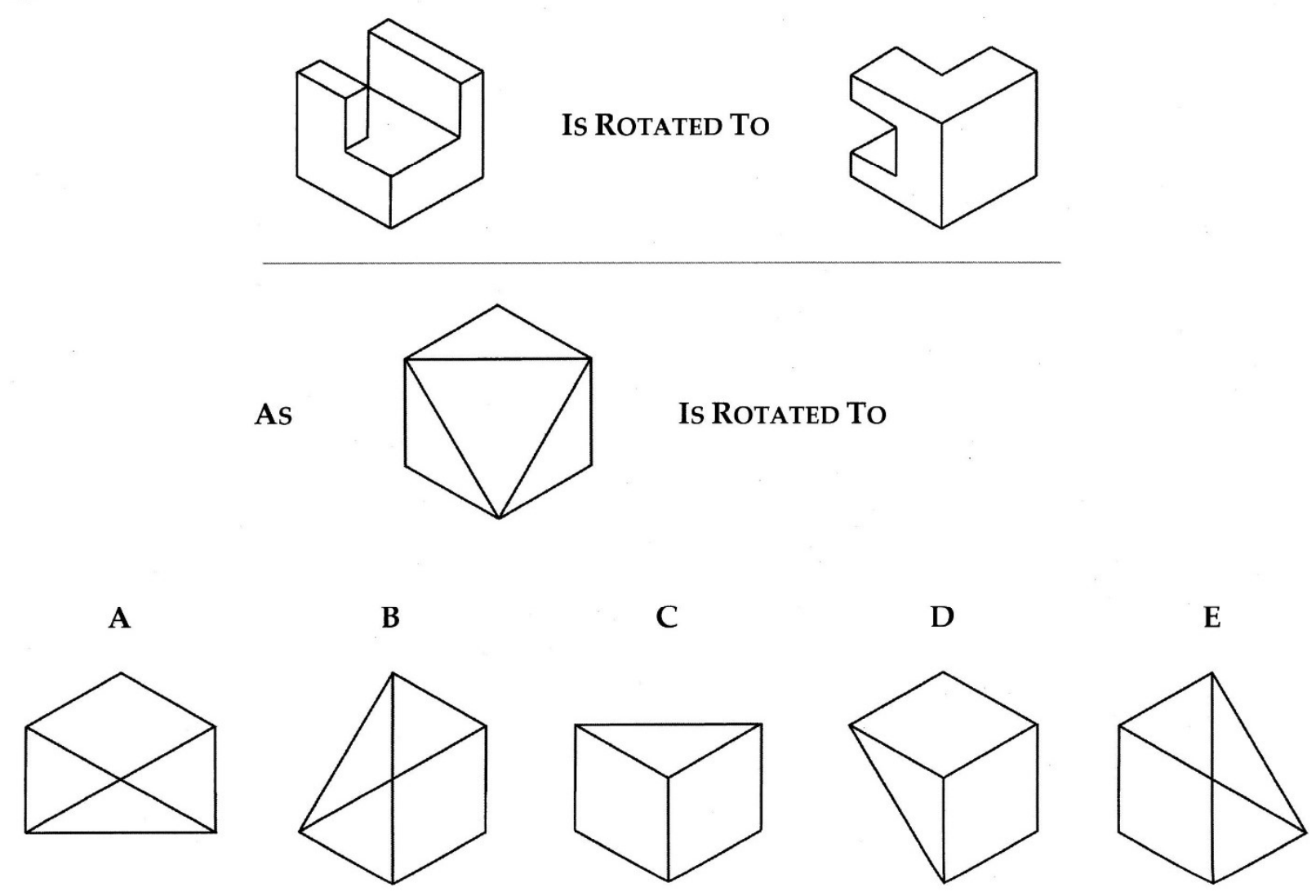


\section{0}
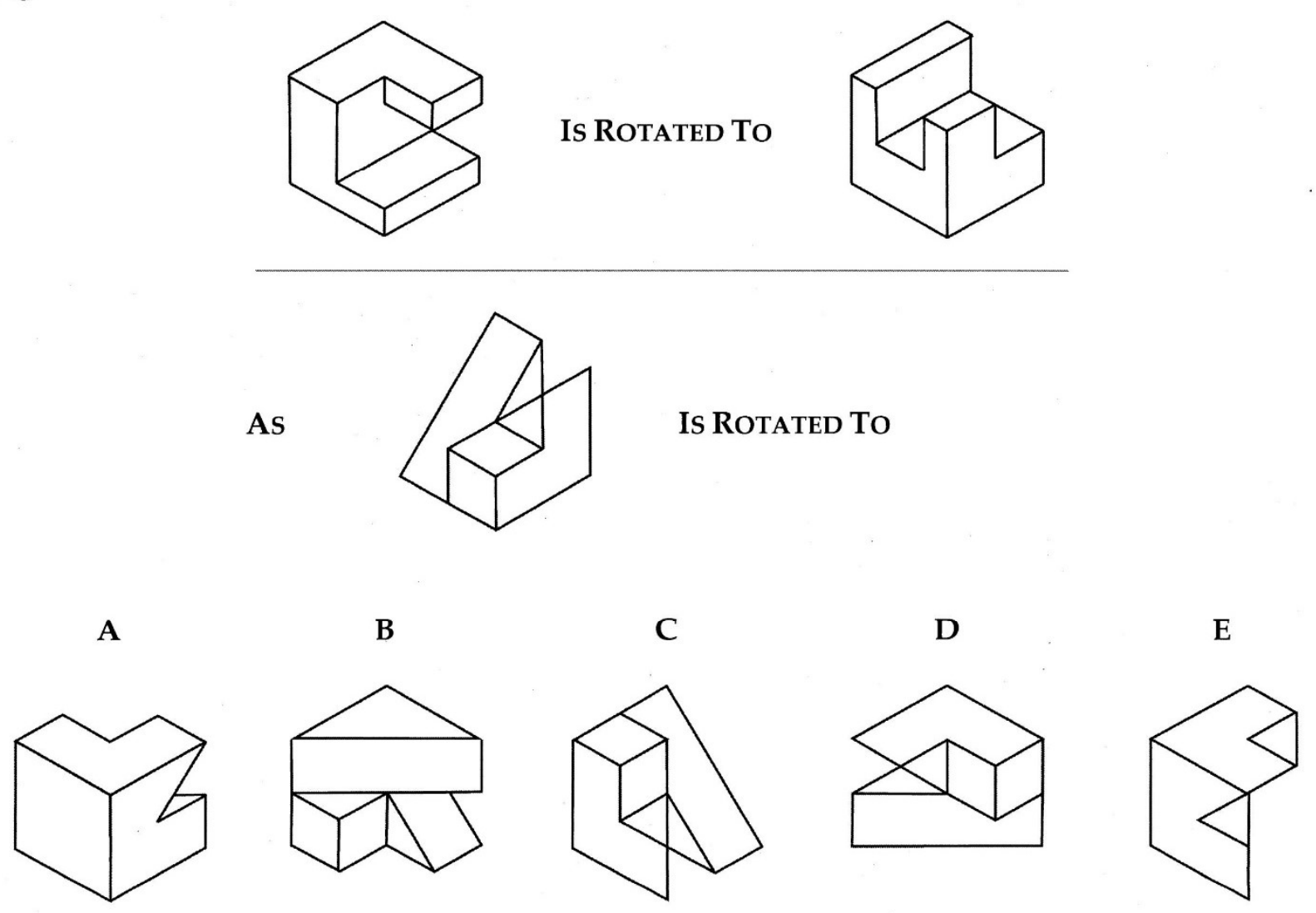
THANK YOU VERY MUCH! 


\section{Revised PSVT:R (2011)}

\section{Answer Key}

$\begin{array}{rrllll}\text { 1. } & \mathrm{B} & 11 . & \mathrm{E} & 21 . & \mathrm{A} \\ \text { 2. } & \mathrm{A} & 12 . & \mathrm{E} & 22 . & \mathrm{D} \\ \text { 3. } & \mathrm{A} & 13 . & \mathrm{B} & 23 . & \mathrm{D} \\ \text { 4. } & \mathrm{D} & 14 . & \mathrm{D} & 24 . & \mathrm{C} \\ \text { 5. } & \mathrm{B} & 15 . & \mathrm{C} & 25 . & \mathrm{D} \\ \text { 6. } & \mathrm{C} & 16 . & \mathrm{E} & 26 . & \mathrm{C} \\ \text { 7. } & \mathrm{E} & 17 . & \mathrm{A} & 27 . & \mathrm{B} \\ \text { 8. } & \mathrm{E} & 18 . & \mathrm{A} & 28 . & \mathrm{E} \\ \text { 9. } & \mathrm{E} & 19 . & \mathrm{B} & 29 . & \mathrm{C} \\ \text { 10. } & \mathrm{D} & 20 . & \mathrm{B} & 30 . & \mathrm{E}\end{array}$




\section{Answer Sheet}

ID:

\begin{tabular}{|c|c|c|c|c|c|}
\hline 1. & (A) (B) (C) (D) () & 11. & (A) (B) (C) (D) (E) & 21. & (A) (B) (C) (D) () \\
\hline 2. & (A) (B) (C) (D) () & 12 . & (A) (B) (C) (D) (E) & 22. & (A) (B) (C) (D) (E) \\
\hline 3. & (A) (B) (C) (D) (E) & 13. & (A) (B) (C) (D) (E) & 23. & (A) (B) (C) (D) (E) \\
\hline 4. & (A) (B) (C) (D) (E) & 14. & (A) (B) (C) (D) (E) & 24. & (A) (B) (C) (D) () \\
\hline 5. & (A) (B) (C) (D) () & 15. & (A) (B) (C) (D) (E) & 25. & (A) (B) (C) (D) (B) \\
\hline 6. & (A) (B) (C) (D) (E) & 16. & (A) (B) (C) (D) (E) & 26. & (A) (B) (C) (D) (E) \\
\hline 7. & (A) (B) (C) (D) () & 17. & (A) (B) (C) (D) (E) & 27. & (A) (B) (C) (D) (E) \\
\hline 8. & (A) (B) (C) (D) (E) & 18. & (A) (B) (C) (D) (E) & 28. & (A) (B) (C) (D) (E) \\
\hline 9. & (A) (B) (C) (D) (E) & 19. & (A) (B) (C) (D) (E) & 29. & (A) (B) (C) (D) (E) \\
\hline . & (A) (B) (C) (D) & 20. & (A) (B) (C) (D) & 30. & (A) (B) (C) (D) \\
\hline
\end{tabular}




\section{Appendix D: Pilot Study 1 Solids of Revolution Problems}

\section{Problem 1}

Find the volume of the solid of revolution obtained by rotating the region bounded by $y=x^{2}+$ $2, x=1, x=4$, and $y=0$ about the $x$-axis.

\section{Problem 2}

Find the volume of the solid of revolution obtained by rotating the region bounded by $y=\sqrt{x}$, $y=0$, and $x=9$ about the $y$-axis.

\section{Problem 3}

Find the volume of the solid of revolution obtained by rotating the region bounded by $y=x$, $y=2 x$, and $x=4$ about the $y$-axis 


\section{Appendix E: Pilot Study 2 Interview Problems}

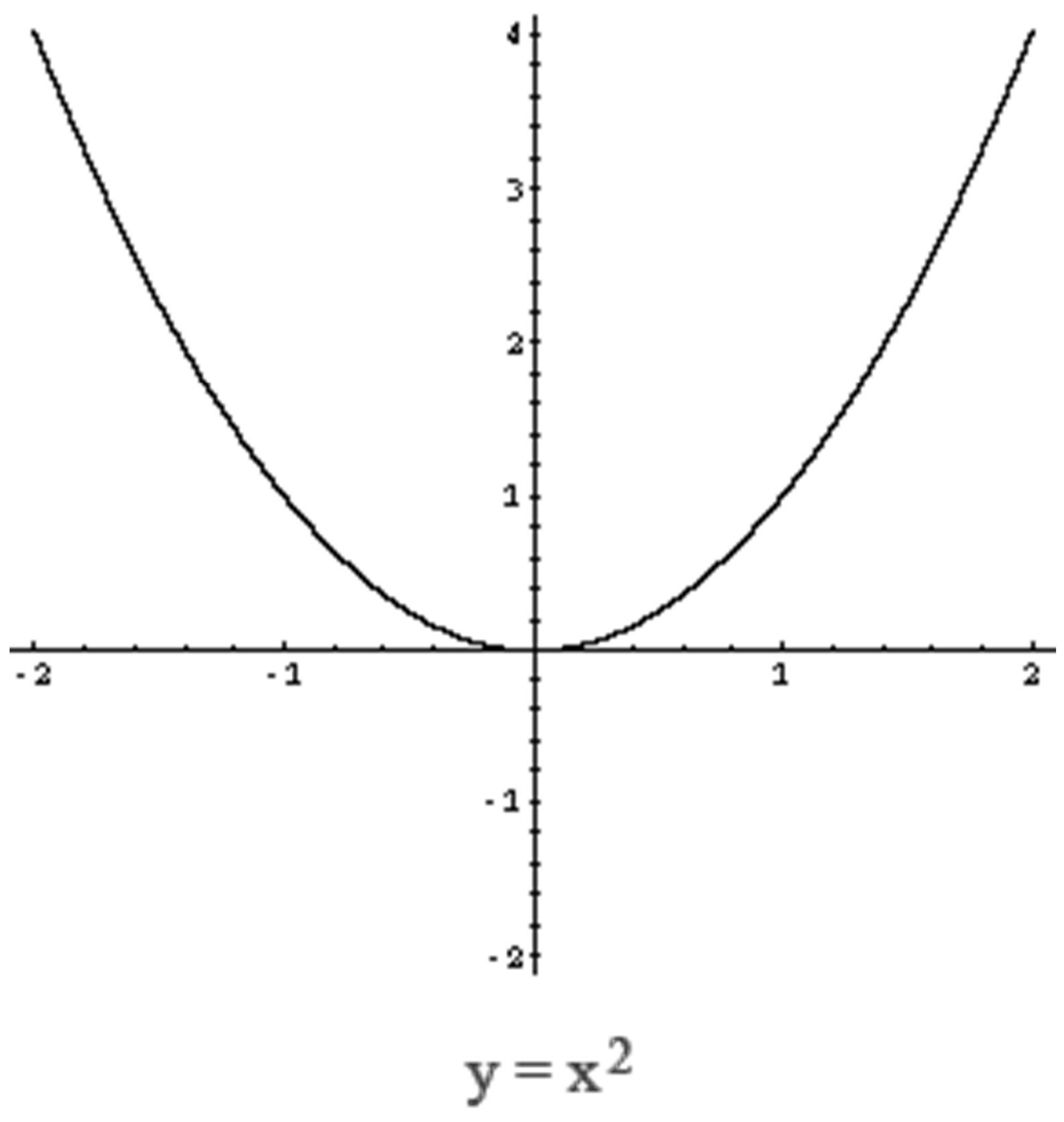

\section{Problem 1}

Take $f(x)=x^{2}, y=1$, and $x=0$ and revolve around the $y$-axis. Draw the picture of this.

\section{Problem 2}

Take $f(x)=x^{2}$ and $x=1$, and $y=0$ and revolve around the $x$-axis. Draw the picture of this.

\section{Problem 3}

Take $\mathrm{f}(\mathrm{x})=\mathrm{x}^{2}, \mathrm{y}=0$, and $\mathrm{x}=1$ and revolve around the line $\mathrm{x}=2$. Draw the picture of this.

\section{Problem 4}

Take $\mathrm{f}(\mathrm{x})=\mathrm{x}^{2}$ and $\mathrm{x}=1$, and $\mathrm{y}=0$ and revolve around the line $\mathrm{y}=2$. Draw the picture of this. 


\section{Appendix F: Interview Protocol}

The interviewer will meet the student in a quiet room with no others in the room. A video camera will be set up to capture the student's responses to the questions and any other important information that the student uses to answer the question, like body language or gestures.

1. Warm-up questions (pre-interview only)
a. Tell me about your math background.
b. What kind of toys did you like to play with when you were growing up?
c. Do you like to do puzzles? What kinds?
d. Did you participate in any sports as a child?

2. Intervention feedback questions (post-interview only)
a. What did you like and/or dislike about the applications/activities?
b. How could the applications/activities be improved?
c. Did you find the use of the haptics device helpful? (Haptic Group only)
d. Did the applications help you learn anything about cutting planes and/or cross- sections?

The interviewer will show the students three questions from the Santa Barbara Solids test that the student has taken previously in the semester.

3. Santa Barbara Solids Test questions 10, 27, 29: This test gives you a 3D shape with a cutting plane and asks you to choose which cross section matches the situation.

a. Do you understand how the cutting plane works?

b. Explain your thought process as you work through each problem.

\section{If a student is having trouble:}

a. Can you eliminate any answers?

b. Try thinking about just one portion of the object (if it is a complex object)

The interviewer will show the student a 3D object, such as a donut, sphere, lightbulb, and ask them to answer the questions below:

\section{Draw the Cross-section questions}


Try to imagine the cross-section that would result if you faced the cutting plane headon, as if you were looking at your reflection in a mirror.

\section{Which way should I cut it? Questions}

Imagine using cutting planes to cut the object several times. Which cutting plane should be used, parallel to the x-axis or parallel to the y-axis, to make it easiest to calculate the area for all cross-sections of the object? Explain your answer.

The interviewer will give the students a piece of paper and pencil and ask the student to do the following.

6. Draw an object with the following properties (pre-interview):

a. The cross-section formed by any vertical cutting plane is a square.

b. The cross-section formed by most (but not all) horizontal cutting planes is a hexagon.

7. Draw an object with the following properties (post-interview):

a. The cross-section formed by most (but not all) vertical cutting planes is an equilateral triangle.

b. The cross-section formed by any horizontal cutting plane is a pentagon. 


\section{Appendix G: Application Directions - With Haptics \\ Application Directions}

\section{Moving from Scene to Scene}

Press the TAB key to move to the next scene.

Press the BACKSPACE key to move to the previous scene.

Moving the Camera

Press the ARROW keys to rotate the camera around the scene.

Press the SPACEBAR to return the camera to its original position.

Viewing Cutting Plane Positions

Press the $\mathbf{X}$ key to display possible cutting planes parallel to the $\mathbf{x}$-axis (horizontal).

Press the $\mathbf{Y}$ key to display possible cutting planes parallel to the $y$-axis (vertical).

Press the SPACEBAR to exit this feature.

Trace the Perimeter

(first, view the possible cutting planes by pressing $X$ or $Y$ )

Press the key $(1,2,3$, etc.) corresponding to the desired cutting plane location to restrict the motion of the haptic device to the perimeter at that location.

Press the SPACEBAR to exit this feature.

Exit

Pres the ESC key to leave the application. 


\section{Appendix H: Application Directions - Without Haptics \\ Application Directions}

Moving from Scene to Scene

Press the TAB key to move to the next scene.

Press the BACKSPACE key to move to the previous scene.

Moving the Camera

Press the ARROW keys to rotate the camera around the scene.

Press the SPACEBAR to return the camera to its original position.

Viewing Cutting Plane Positions

Press the $\mathbf{X}$ key to display possible cutting planes parallel to the $\mathbf{X}$-axis (horizontal).

Press the $\mathbf{Y}$ key to display possible cutting planes parallel to the $\mathrm{x}$-axis (vertical).

Press the SPACEBAR to exit this feature.

Exit

Pres the ESC key to leave the application. 


\section{Appendix I: Sample Activity}

\section{Scene 1: Cylinder}

\section{Explore the scene by moving the camera to view all angles of the object.}

1. Draw a 3-D sketch of the object (do the best you can).

2. Press the $\mathbf{X}$ key to view a few horizontal cutting plane locations. Draw a picture of the cross-section created by each cutting plane. Try to imagine the cross-section that would result if you faced the cutting plane head-on, as if you were looking at your reflection in a mirror. Try to make the cross-sections proportional to each other.

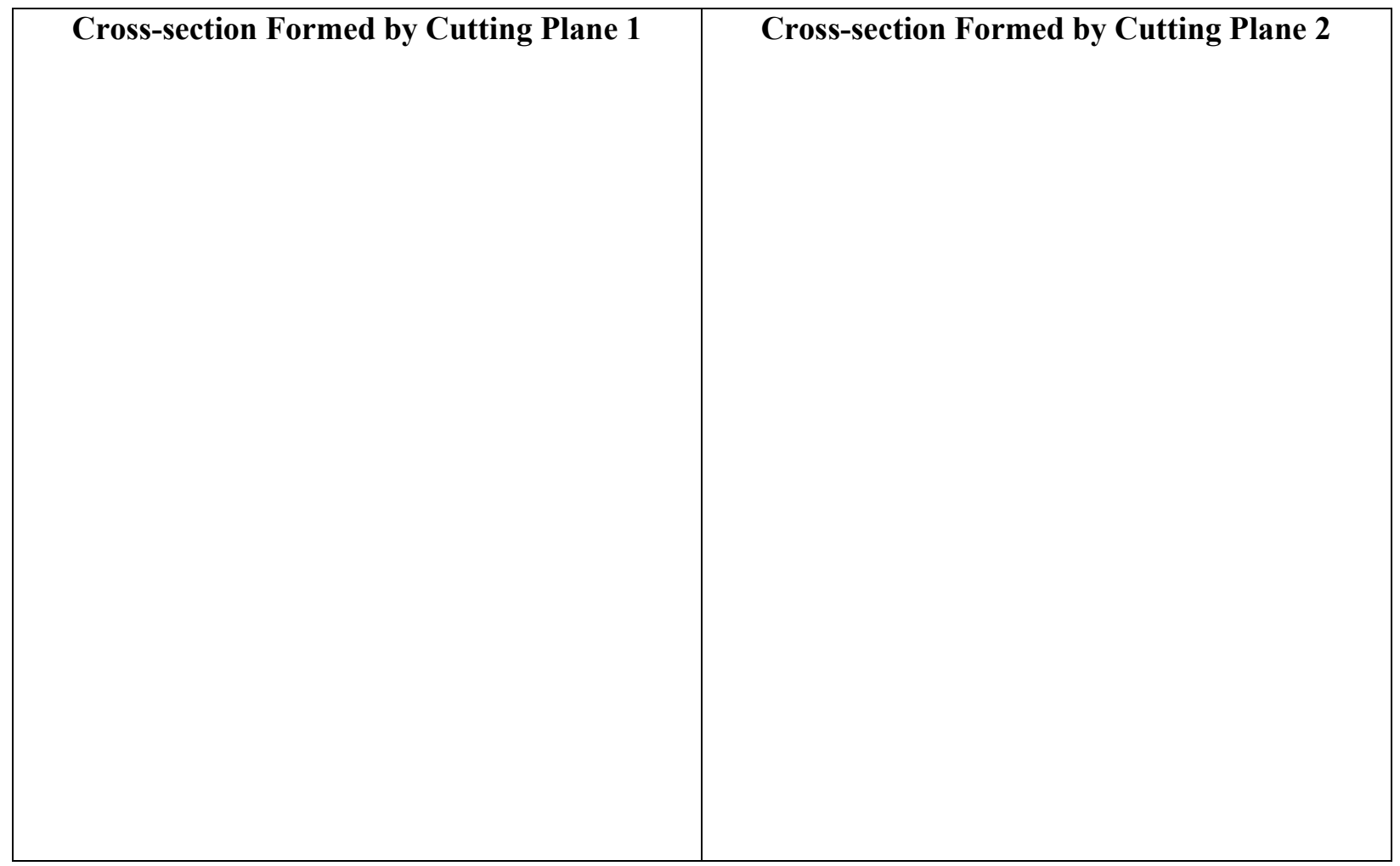


3. Press the $\mathbf{Y}$ key to view a few vertical cutting plane locations. Draw a picture of the cross-section created by each cutting plane. Try to imagine the cross-section that would result if you faced the cutting plane head-on, as if you were looking at your reflection in a mirror. Try to make the cross-sections proportional to each other.

\begin{tabular}{|l|l|}
\hline Cross-section Formed by Cutting Plane 3 & Cross-section Formed by Cutting Plane 4 \\
& \\
& \\
\hline
\end{tabular}

4. Imagine using cutting planes to cut the object several times (more than the choices shown in 2 and 3). Which cutting plane should be used (parallel to $\mathrm{x}$-axis or parallel to $\mathrm{y}$-axis) to make it easiest to calculate the area for all cross-sections of the object? Explain your answer. 
5. Based on your answer in 4, what measurements on the 3-D object would you need to be able to calculate the area of the cross-section? Explain your answer. 


\section{Scene 2: Lightbulb}

Explore the scene by moving the camera to view all angles of the object.

1. Draw a 3-D sketch of the object (do the best you can).

2. Press the $\mathbf{X}$ key to view a few horizontal cutting plane locations. Draw a picture of the cross-section created by each cutting plane. Try to imagine the cross-section that would result if you faced the cutting plane head-on, as if you were looking at your reflection in a mirror. Try to make the cross-sections proportional to each other.

\begin{tabular}{|l|l|}
\hline Cross-section Formed by Cutting Plane 1 & Cross-section Formed by Cutting Plane 2 \\
& \\
& \\
\hline
\end{tabular}


3. Press the $\mathbf{Y}$ key to view a few vertical cutting plane locations. Draw a picture of the cross-section created by each cutting plane. Try to imagine the cross-section that would result if you faced the cutting plane head-on, as if you were looking at your reflection in a mirror. Try to make the cross-sections proportional to each other.

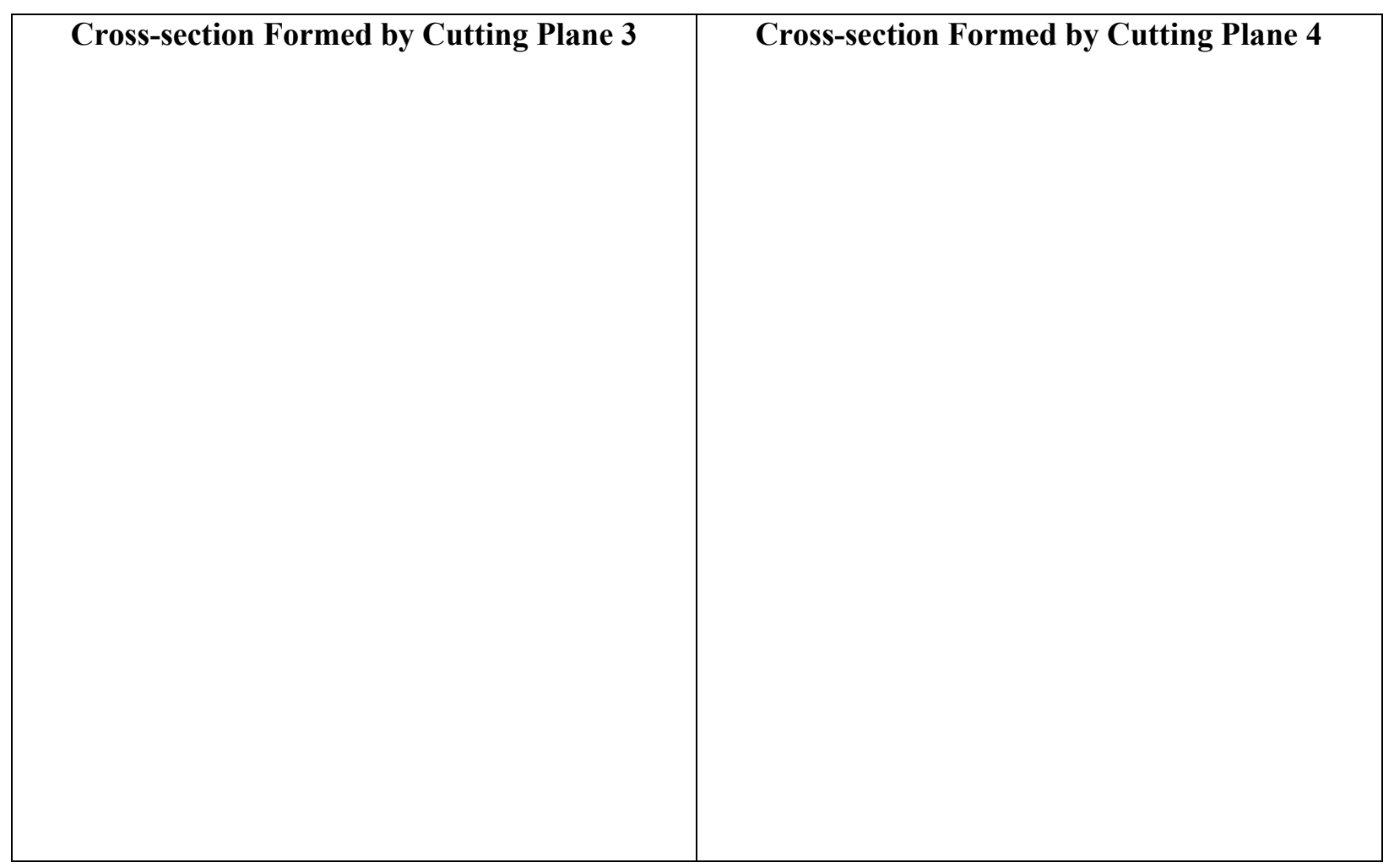

4. Imagine using cutting planes to cut the object several times (more than the choices shown in 2 and 3). Which cutting plane should be used (parallel to x-axis or parallel to y-axis) to make it easiest to calculate the area for all cross-sections of the object? Explain your answer.

5. Based on your answer in 4, what measurements on the 3-D object would you need to be able to calculate the area of the cross-section? Explain your answer. 


\section{Scene 3: Pyramid}

Explore the scene by moving the camera to view all angles of the object.

1. Draw a 3-D sketch of the object (do the best you can).

2. Press the $\mathbf{X}$ key to view a few horizontal cutting plane locations. Draw a picture of the cross-section created by each cutting plane. Try to imagine the cross-section that would result if you faced the cutting plane head-on, as if you were looking at your reflection in a mirror. Try to make the cross-sections proportional to each other.

\begin{tabular}{|l|l|}
\hline Cross-section Formed by Cutting Plane 1 & Cross-section Formed by Cutting Plane 2 \\
& \\
& \\
\hline
\end{tabular}


3. Press the $\mathbf{Y}$ key to view a few vertical cutting plane locations. Draw a picture of the cross-section created by each cutting plane. Try to imagine the cross-section that would result if you faced the cutting plane head-on, as if you were looking at your reflection in a mirror. Try to make the cross-sections proportional to each other.

\begin{tabular}{|l|l|}
\hline Cross-section Formed by Cutting Plane 3 & Cross-section Formed by Cutting Plane 4 \\
& \\
& \\
\hline
\end{tabular}

4. Imagine using cutting planes to cut the object several times (more than the choices shown in 2 and 3). Which cutting plane should be used (parallel to x-axis or parallel to y-axis) to make it easiest to calculate the area for all cross-sections of the object? Explain your answer.

5. Based on your answer in 4, what measurements on the 3-D object would you need to be able to calculate the area of the cross-section? Explain your answer. 